University of Louisville

ThinkIR: The University of Louisville's Institutional Repository

Electronic Theses and Dissertations

$8-2020$

\title{
Search for new physics using lepton flavor violating signatures in modern particle colliders.
}

Atanu Pathak

University of Louisville

Follow this and additional works at: https://ir.library.louisville.edu/etd

Part of the Elementary Particles and Fields and String Theory Commons

\section{Recommended Citation}

Pathak, Atanu, "Search for new physics using lepton flavor violating signatures in modern particle colliders." (2020). Electronic Theses and Dissertations. Paper 3526.

https://doi.org/10.18297/etd/3526

This Doctoral Dissertation is brought to you for free and open access by ThinkIR: The University of Louisville's Institutional Repository. It has been accepted for inclusion in Electronic Theses and Dissertations by an authorized administrator of ThinkIR: The University of Louisville's Institutional Repository. This title appears here courtesy of the author, who has retained all other copyrights. For more information, please contact thinkir@louisville.edu. 


\title{
SEARCH FOR NEW PHYSICS USING LEPTON FLAVOR VIOLATING SIGNATURES IN MODERN PARTICLE COLLIDERS
}

\author{
By \\ Atanu Pathak \\ A Dissertation Submitted to the Faculty of the \\ College of Arts and Sciences of the University of Louisville \\ in Partial Fulfillment of the Requirements for the Degree of
}

Doctor of Philosophy in Physics

Department of Physics and Astronomy

University of Louisville

Louisville, Kentucky

August 2020 
(C) Atanu Pathak 2020

All Rights Reserved 



\section{SEARCH FOR NEW PHYSICS USING LEPTON FLAVOR VIOLATING SIGNATURES IN MODERN PARTICLE COLLIDERS}

By

\section{Atanu Pathak}

A Dissertation Approved on

August 3rd, 2020

By the following Dissertation Committee

Dissertation Chair

Dr. Swagato Banerjee

Dissertation Co-Chair (if applicable)

Dr. David N. Brown

Dr. Chris L. Davis

Dr. Thomas Riedel 
For all the people 


\section{ACKNOWLEDGEMENTS}

First and foremost I would like to thank my family, especially my parents, without the love and support of whom I would not have gotten to where I am today.

I would like to thank Prof. Swagato Banerjee for giving me the opportunity to work with him. I am grateful for his help and support through all the years of my Ph.D. program. I count myself lucky to have had an amazing mentor like him.

I thank all members of my Ph.D. thesis committee: Prof. David N. Brown, Prof. Chris L. Davis and Prof. Thomas Riedel. Their engagement with my research and guidance significantly improved my knowledge as well as the quality of this thesis.

Special thanks to Diptaparna Biswas for many enlightening discussions and help towards my Ph.D. program.

I thank all the other Professors, my fellow graduate students, colleagues, and friends in the Department of Physics and Astronomy at the University of Louisville.

I am very thankful to the ATLAS and Belle II collaborations for giving me the opportunity to successfully perfrom this research.

Last, but not least, I would like to take the opportunity to thank all the people I had the chance to work with during my Ph.D. program. 


\begin{abstract}
SEARCH FOR NEW PHYSICS USING LEPTON FLAVOR VIOLATING SIGNATURES IN MODERN PARTICLE COLLIDERS

Atanu Pathak
\end{abstract}

August 3rd, 2020

Charged lepton flavor violation is a clear signal of new physics. Such decays are not allowed in the Standard Model, but highly anticipated in a large class of new physics models. The ATLAS and the CMS experiments at the Large Hadron Collider are only two places where we may discover lepton-flavor-violating decays of the Higgs boson in the near future. With an increase in the center-of-mass energy at the Large Hadron Collider (LHC) from 7 and $8 \mathrm{TeV}$ in Run1 (2011-2012) to $13 \mathrm{TeV}$ in Run2 (2015-2018), the rate of production of the Higgs boson has increased. This makes the search for lepton flavor violating decays of the Higgs boson at the worlds highest energy particle collider very interesting. Indirect expectations based on current upper limits on the branching fraction of the lepton flavor violating decays of the $\tau$ lepton to an electron or a muon plus a gamma in the final state at the $10^{-8}$ level imply bounds on lepton flavor violating decays of the Higgs boson at the $10 \%$ level.

A direct search for lepton flavor violation in decays of the Higgs boson with the ATLAS detector at the LHC is presented here. The analysis is performed in the $H \rightarrow l \tau$ channel, where the leading lepton $(\ell)$ can be either an electron or a muon, 
and the $\tau$ lepton decays into an opposite flavored lighter lepton or via a hadronic decay channel. Decays modes where the prompt lepton from Higgs and from the $\tau$ lepton are both electrons or muons are not considered in the final state because of large irreducible background from di-leptonic decays of the $\mathrm{Z}$ boson.

Published results of this search are presented in this thesis based on a data sample of proton-proton collisions collected by the ATLAS detector corresponding to an integrated luminosity of 36 inverse femtobarns $\left(\mathrm{fb}^{-1}\right)$ at a center-of-mass energy $(\sqrt{s})$ of 13 trillion-electron-volts (TeV) during the 2015-2016 data-taking period (Run2). The analysis is found to be three times more sensitive than the previous analysis performed with $20 \mathrm{fb}^{-1}$ of data collected at $\sqrt{s}=8 \mathrm{TeV}$ during the 2012 data-taking period (Run1). The improvements mainly arise from inclusion of additional kinematic regions in the analysis with higher sensitivity to signal, as well as an improved technique to understand the modelling of the fake-background with a data-driven method using a transfer factor from control region to signal region. Further improvements are obtained by inclusion of a new phase-space region corresponding to the Vector Boson Fusion production mechanism of the Higgs boson, as well as use of multi-variate signal-to-background discriminants. The result is already systematic error dominated and thus will not improve significantly by adding more data at the Large Hadron Collider. The upper limits obtained by direct search for lepton flavor violating decays of the Higgs boson obtained with the present analysis at the Large Hadron Collider are about twenty-five times lower than the indirect prediction on the respective branching fractions.

To complement my research goal of searching for new physics with lepton flavor violating signatures in final states containing the $\tau$ lepton, I also studied the generator level modeling of decays of the $\tau$ lepton decaying into Standard Model processes at the Belle II experiment at the worlds highest luminosity SuperKEKB collider in Japan. This validation work involves benchmarking of the visible mass distributions from 
each one of the allowed final states in the Standard Model decays of the $\tau$ lepton

On the technical side, to obtain authorship qualification on the ATLAS experiment, I have worked on the Inner Tracker (ITk) upgrade. I mapped the material budget of the detector in terms of the radiation length traversed for the current Inner Detector geometry as well as the proposed ITk layout to be deployed at the Phase II upgrade of the ATLAS experiment. The materials are separately categorized in terms of a detailed description of cryogenic, electrical, and mechanical support structures. I implemented the modeling of the mechanical supports and electrical cabling to be used in central simulation and incorporated it into a global database. I performed benchmarking studies of timing requirements of the charged particle reconstruction as tracks in the Inner Detector currently used in ATLAS versus the new layout of an upgraded ITk.

To obtain authorship qualification on the Belle II experiment, my technical contributions are related to the K-long and muon detector (KLM). I am the troubleshooting expert and data-quality monitoring (DQM) liaison of the KLM detector of the Belle II experiment. I built a web interface, which generates web-pages dynamically, to monitor the performance of the detector, which are crucial to diagnosing changes in the machine beam background and track changes in the thresholds settings, calibration constants, and trigger settings. 


\section{TABLE OF CONTENTS}

DEDICATION .......................

ACKNOWLEDGEMENTS ................... iv

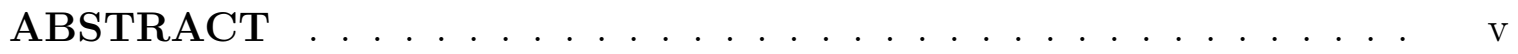

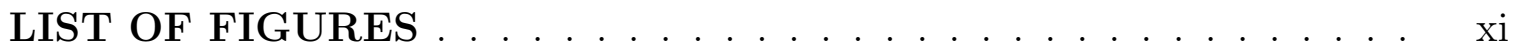

LIST OF TABLES $\ldots \ldots \ldots \ldots \ldots \ldots \ldots \ldots \ldots \ldots \ldots$ xvi

\section{CHAPTERS}

I. OUTLINE OF THE THESIS . . . . . . . . . . . . . 1

II. STANDARD MODEL OF PARTICLE PHYSICS . . . . . . 3

2.1 Noether's Theorem . . . . . . . . . . . . . . . . . . . 4

2.2 Dirac Lagrangian . . . . . . . . . . . . . . . . . . . . 5

2.3 Quantum Electrodynamics . . . . . . . . . . . . 6

2.4 Electroweak Unification . . . . . . . . . . . . . . . . 9

2.5 Higgs Mechanism . . . . . . . . . . . . . . . . . . . 14

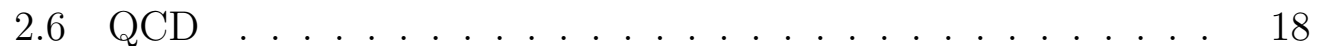

2.7 Higgs boson at the LHC . . . . . . . . . . . . . . . 18

2.8 Lepton flavour violating decays . . . . . . . . . . . . . 24

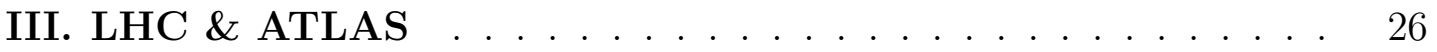

3.1 The Large Hadron Collider . . . . . . . . . . . . . . . . . 26

3.2 ATLAS Detector . . . . . . . . . . . . . . . . 29

3.2 .1 Inner Detector . . . . . . . . . . . . . . . . . 31

3.2 .2 Calorimeter . . . . . . . . . . . . . . . . . 32

3.2 .3 Muon Spectrometer . . . . . . . . . . . . . . . . 34

3.3 Trigger System . . . . . . . . . . . . . . . . . . . . . 34 
IV. ITk UPGRADE OF THE ATLAS EXPERIMENT

4.1 Introduction . . . . . . . . . . . . . . . . . 38

4.2 The ATLAS Inner Tracker(ITk) Layout . . . . . . . . . . . 40

4.3 Simulation and Track Reconstruction . . . . . . . . . . . 42

4.3 .1 Simulation samples . . . . . . . . . . . . . . . . 42

4.3.2 Track Reconstruction . . . . . . . . . . . . . . . . . 44

4.3 .3 CPU Timing . . . . . . . . . . . . . . . . . . . . 45

V. BELLL II DETECTOR . . . . . . . . . . . . . . . 54

5.1 Introduction . . . . . . . . . . . . . . . . 54

5.2 Belle II detector . . . . . . . . . . . . . . . . . 55

5.2 .1 Vertex detector $(\mathrm{VXD}) \ldots \ldots \ldots \ldots 5$

5.2 .2 Central Drift Chamber (CDC) . . . . . . . . . 58

5.2.3 Particle identification system (TOP and ARICH) . . $\quad 59$

5.2.4 Electromagnetic Calorimeter (ECL) . . . . . . . . . 61

$5.2 .5 \quad K_{\mathrm{L}^{-}}$Muon Detector $(\mathrm{KLM}) \ldots \ldots \ldots$

5.3 Trigger System . . . . . . . . . . . . . . . . . . . . 62

VI. DATA QUALITY MONITORING OF THE MUON DETEC-

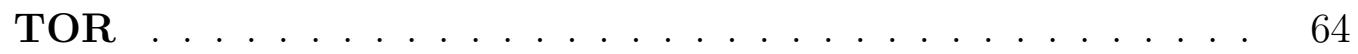

6.1 Introduction . . . . . . . . . . . . . . . . . . 64

6.2 Structure of the KLM Detector . . . . . . . . . . . . . 64

6.3 KLM Data Quality . . . . . . . . . . . . . . . . . . 67

6.4 Expert-level DQM webpages for KLM . . . . . . . . . 69

VII. MODELLING OF $\tau$ DECAYS AT THE BELLE II EXPER-

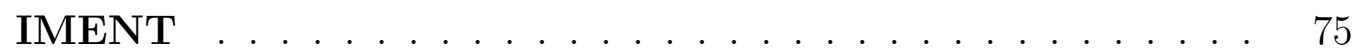

$7.1 \tau$ decays . . . . . . . . . . . . . . . . . 75

7.2 TAUOLA-BBB Monte Carlo . . . . . . . . . . . . . . . 78

7.3 Modelling of visible mass in $\tau$ decays . . . . . . . . . . 82

VIII. SEARCHES FOR LEPTON-FLAVOR-VIOLATING DECAYS OF THE HIGGS BOSON WITH THE ATLAS EXPERIMENT 99

8.1 Introduction . . . . . . . . . . . . . . . . . . . . . 99

8.2 Data Samples and Monte Carlo Simulation . . . . . . . . 101

8.2 .1 Simulation samples . . . . . . . . . . . . . . . . 101

8.3 Object Reconstruction . . . . . . . . . . . . . . . . . . . 104

8.3.1 Electrons . . . . . . . . . . . . . . . . . . . . 104

8.3 .2 Muons . . . . . . . . . . . . . . . . . . . 104 
8.3.3 Jets . . . . . . . . . . . . . . . . . . . . . . . . . . . 105

8.3.4 $\tau$ decays . . . . . . . . . . . . . . . . . . 105

8.3.5 Missing transverse energy . . . . . . . . . . . . 106

8.3 .6 Overlap removal . . . . . . . . . . . . . . . . . . . . 106

8.3.7 $\ell \tau$ mass reconstruction . . . . . . . . . . . . . . . . 107

8.3 .8 BDT variable . . . . . . . . . . . . . . . . . 110

8.4 Event Selection and Categorization . . . . . . . . . . . . 115

8.5 Background modelling . . . . . . . . . . . . . . . 120

8.5.1 $\ell \tau_{\ell^{\prime}}$ channel . . . . . . . . . . . . . . . . . . 120

$8.5 .2 \ell \tau_{\text {had }}$ channel . . . . . . . . . . . . . . . . . 122

8.6 Systematic uncertainties and pre-fit results . . . . . . . 137

8.6.1 Sources of systematics . . . . . . . . . . . . . 137

8.6 .2 Pre-fit results . . . . . . . . . . . . . . . . . . . . . . . . . 143

8.7 Statistical analysis . . . . . . . . . . . . . . . . 146

8.8 Results and combined sensitivity . . . . . . . . . . . . 151

8.9 Conclusions . . . . . . . . . . . . . . . . . . . . 157

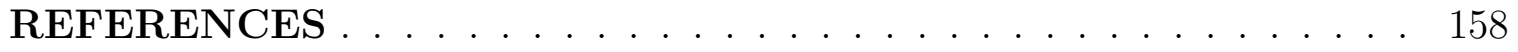

CURRICULUM VITA . . . . . . . . . . . . . . . . . . 175 


\section{LIST OF FIGURES}

\section{Figure}

2.1 Feynman diagrams for the Higgs production modes accessible at the $\mathrm{LHC} \ldots \ldots \ldots \ldots \ldots \ldots$

2.2 Production cross section for various production modes as a function of the Higgs mass $m_{H}$ for both 8 and $13 \mathrm{TeV}$ center-of-mass energies 21

2.3 Possible Higgs boson decays in the SM . . . . . . . . . . . . . . . . 22

2.4 Branching ratio for various decay modes as a function of the Higgs mass $m_{H}$. The range of the left plot has an upper bound of $200 \mathrm{GeV}$ to reveal the structure of the curves at low $m_{H} \ldots \ldots$. . . . .

3.1 Layout of the LHC ring showing the positions of each experiment at the collision points as well as injection, beam dump, and beam cleaning regions . . . . . . . . . . . . . . . 27

3.2 Delivered Luminosity versus time for 2011-2018 . . . . . . . . . . . 29

3.3 Layout of the ATLAS detector showing the major subdetectors . . . 30

3.4 Layout of the inner detector in the barrel showing the Pixel, SCT, and TRT detectors and their geometrical configuration . . . . . . . 31

3.5 Layout of the calorimetry system . . . . . . . . . . . . . . . 34

3.6 Cross section of the muon spectrometer in both barrel and end-cap 35

3.7 The observed rates per trigger are reported at a luminosity of $1.7 \times$ $10^{34} \mathrm{~cm}^{-2} \mathrm{~s}^{-1}$ and $\langle\mu\rangle \simeq 50$ as raw rates, including rate overlapping between triggers . . . . . . . . . . . . . . . . . . 37

4.1 Schedule for High Luminosity LHC (HL-LHC) upgrade . . . . . . . 39

$4.2 \quad$ More primary vertices seen due to higher pile-up. . . . . . . . . . . 40

4.3 3D split view of the ATLAS Phase-II Inner Tracker ITk with the Inclined Duals detector layout . . . . . . . . . . . . . . . . . . . . 41

$4.4 \quad R$ vs. $z$ layout of ITk (left) and ID-Run2 (right). . . . . . . . . . . . 41

4.5 Location of the material for one quadrant of the ITk Layout. The Pixel detector is shown in red, and the strip detector in blue . . . . 43

4.6 Material map of ITk (left) and ID-Run2 (right) vs. $\eta$. . . . . . . . 43

4.7 Reconstruction times for ITk . . . . . . . . . . . . . 50

4.8 Reconstruction times for ID-Run2 . . . . . . . . . . . 50

4.9 CPU benchmarking summary plot for ITk and ID-Run2 . . . . . . 51

5.1 Belle II top view . . . . . . . . . . . . . . . . . 56 
5.2 A schematic view of the Belle II vertex detector with a Be beam pipe, two pixelated layers and four layers with silicon strip sensors . . . .

5.3 A cosmic muon as recorded by the Belle II Central Drift Chamber

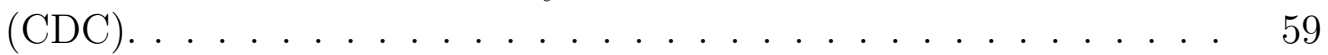

5.4 One of the 16 modules of the TOP detector $\ldots \ldots \ldots$

5.5 Photon detector plane with HAPD sensors of the ARICH . . . . . . 61

6.1 Schematics of the barrel and endcap sections of the KLM detector . 65

6.2 Side view of the KLM detector . . . . . . . . . . . . . . 66

6.3 RawKLMs for Barrel and Endcap . . . . . . . . . . . . . . . . 68

$6.4 \quad$ Sector occupancy for Barrel and Endcap . . . . . . . . . . . . . 69

6.5 Hit occupancy for Barrel in $x$ vs $y$ view . . . . . . . . . . . 70

$6.6 \quad$ Hit occupancy for Endcap backward in $x$ vs $y$ view $\ldots \ldots . . .71$

$6.7 \quad$ Hit occupancy for Endcap forward in $x$ vs $y$ view $\ldots \ldots \ldots . . .72$

6.8 Expert-level DQM webpages for KLM. . . . . . . . . . . . . 74

$7.1 \quad m_{v i s}$ for $\tau^{-} \rightarrow e^{-} \bar{\nu}_{e} \nu_{\tau} \ldots \ldots \ldots \ldots \ldots \ldots$

$7.2 \quad m_{v i s}$ for $\tau^{-} \rightarrow e^{-} e^{-} e^{+} \bar{\nu}_{e} \nu_{\tau} \ldots \ldots \ldots \ldots \ldots \ldots$

$7.3 \quad m_{v i s}$ for $\tau^{-} \rightarrow \mu^{-} \bar{\nu}_{\mu} \nu_{\tau} \ldots \ldots \ldots \ldots \ldots \ldots \ldots$

$7.4 m_{v i s}$ for $\tau^{-} \rightarrow \pi^{-} \nu_{\tau} \ldots \ldots \ldots \ldots \ldots \ldots$

$7.5 \quad m_{v i s}$ for $\tau^{-} \rightarrow \rho^{-}\left(\rightarrow \pi^{-} \gamma\right) \nu_{\tau} \ldots \ldots \ldots \ldots \ldots$

$7.6 \quad m_{v i s}$ for $\tau^{-} \rightarrow a_{1}^{-}\left(\rightarrow \pi^{-} \gamma\right) \nu_{\tau} \ldots \ldots \ldots \ldots \ldots$

$7.7 \quad m_{v i s}$ for $\tau^{-} \rightarrow K^{-} \nu_{\tau} \ldots \ldots \ldots \ldots \ldots \ldots$

$7.8 \quad m_{v i s}$ for $\tau^{-} \rightarrow \pi^{-} \pi^{0} \nu_{\tau} \ldots \ldots \ldots \ldots \ldots \ldots$

$7.9 \quad m_{v i s}$ for $\tau^{-} \rightarrow \pi^{-} \omega\left(\rightarrow \pi^{0} \gamma\right) \nu_{\tau} \ldots \ldots \ldots \ldots \ldots \ldots$

$7.10 \quad m_{v i s}$ for $\tau^{-} \rightarrow K^{-} \pi^{0} \nu_{\tau} \ldots \ldots \ldots \ldots \ldots \ldots$

$7.11 \quad m_{v i s}$ for $\tau^{-} \rightarrow \pi^{-} 2 \pi^{0} \nu_{\tau} \ldots \ldots \ldots \ldots \ldots \ldots$

$7.12 \quad m_{v i s}$ for $\tau^{-} \rightarrow \pi^{-} \pi^{0} \omega\left(\rightarrow \pi^{0} \gamma\right) \nu_{\tau} \ldots \ldots \ldots \ldots \ldots$

$7.13 \quad m_{v i s}$ for $\tau^{-} \rightarrow K^{-} 2 \pi^{0} \nu_{\tau} \ldots \ldots \ldots \ldots \ldots \ldots \ldots$

$7.14 \quad m_{v i s}$ for $\tau^{-} \rightarrow \pi^{-} 3 \pi^{0} \nu_{\tau} \ldots \ldots \ldots \ldots \ldots \ldots \ldots$

$7.15 \quad m_{v i s}$ for $\tau^{-} \rightarrow K^{-} 3 \pi^{0} \nu_{\tau} \ldots \ldots \ldots \ldots \ldots \ldots$

$7.16 \quad m_{v i s}$ for $\tau^{-} \rightarrow \pi^{-} 4 \pi^{0} \nu_{\tau} \ldots \ldots \ldots \ldots \ldots \ldots$

$7.17 \quad m_{v i s}$ for $\tau^{-} \rightarrow \pi^{-} K_{S}^{0} \nu_{\tau} \ldots \ldots \ldots \ldots \ldots \ldots$

$7.18 m_{v i s}$ for $\tau^{-} \rightarrow \pi^{-} K_{L}^{0} \nu_{\tau} \ldots \ldots \ldots \ldots \ldots \ldots \ldots$

$7.19 m_{v i s}$ for $\tau^{-} \rightarrow K^{-} K_{S}^{0} \nu_{\tau} \ldots \ldots \ldots \ldots \ldots \ldots \ldots$

$7.20 \quad m_{v i s}$ for $\tau^{-} \rightarrow K^{-} K_{L}^{0} \nu_{\tau} \ldots \ldots \ldots \ldots \ldots \ldots$

$7.21 \quad m_{v i s}$ for $\tau^{-} \rightarrow \pi^{-} K_{S}^{0} \pi^{0} \nu_{\tau} \ldots \ldots \ldots \ldots \ldots \ldots \ldots$

$7.22 \quad m_{v i s}$ for $\tau^{-} \rightarrow \pi^{-} K_{L}^{0} \pi^{0} \nu_{\tau} \ldots \ldots \ldots \ldots \ldots \ldots \ldots$

$7.23 \quad m_{v i s}$ for $\tau^{-} \rightarrow K^{-} \pi^{0} K_{S}^{0} \nu_{\tau} \ldots \ldots \ldots \ldots \ldots \ldots$

$7.24 \quad m_{v i s}$ for $\tau^{-} \rightarrow K^{-} \pi^{0} K_{L}^{0} \nu_{\tau} \ldots \ldots \ldots \ldots \ldots \ldots$

$7.25 \quad m_{v i s}$ for $\tau^{-} \rightarrow \pi^{-} 2 \pi^{0} K_{S}^{0} \nu_{\tau} \ldots \ldots \ldots \ldots \ldots \ldots$

$7.26 \quad m_{v i s}$ for $\tau^{-} \rightarrow \pi^{-} 2 \pi^{0} K_{L}^{0} \nu_{\tau} \ldots \ldots \ldots \ldots \ldots \ldots \ldots$

$7.27 \quad m_{v i s}$ for $\tau^{-} \rightarrow \pi^{-} K_{S}^{0} K_{S}^{0} \nu_{\tau} \ldots \ldots \ldots \ldots \ldots \ldots$

$7.28 \quad m_{v i s}$ for $\tau^{-} \rightarrow \pi^{-} K_{L}^{0} K_{L}^{0} \nu_{\tau} \ldots \ldots \ldots \ldots \ldots$

$7.29 \quad m_{v i s}$ for $\tau^{-} \rightarrow \pi^{-} K_{S}^{0} K_{L}^{0} \nu_{\tau} \ldots \ldots \ldots \ldots \ldots$

$7.30 \quad m_{v i s}$ for $\tau^{-} \rightarrow \pi^{-} \pi^{0} K_{S}^{0} K_{S}^{0} \nu_{\tau} \ldots \ldots \ldots \ldots \ldots \ldots$

$7.31 \quad m_{v i s}$ for $\tau^{-} \rightarrow \pi^{-} \pi^{0} K_{L}^{0} K_{L}^{0} \nu_{\tau} \ldots \ldots \ldots \ldots \ldots \ldots$ 
$7.32 \quad m_{v i s}$ for $\tau^{-} \rightarrow \pi^{-} \pi^{0} K_{S}^{0} K_{L}^{0} \nu_{\tau} \ldots \ldots \ldots \ldots \ldots \ldots$

$7.33 \quad m_{v i s}$ for $\tau^{-} \rightarrow \pi^{-} \pi^{0} \eta(\rightarrow \gamma \gamma) \nu_{\tau} \ldots \ldots \ldots \ldots \ldots \ldots$

$7.34 \quad m_{v i s}$ for $\tau^{-} \rightarrow \pi^{-} \pi^{0} \eta\left(\rightarrow \pi^{0} \pi^{0} \pi^{0}\right) \nu_{\tau} \ldots \ldots \ldots \ldots$. . . . . . 89

$7.35 \quad m_{\text {vis }}$ for $\tau^{-} \rightarrow \pi^{-} \pi^{0} \eta\left(\rightarrow \pi^{+} \pi^{-} \pi^{0}\right) \nu_{\tau} \ldots \ldots \ldots \ldots \ldots \ldots$

$7.36 \quad m_{v i s}$ for $\tau^{-} \rightarrow \pi^{-} \pi^{0} \eta\left(\rightarrow \pi^{+} \pi^{-} \gamma\right) \nu_{\tau} \ldots \ldots \ldots \ldots \ldots$

$7.37 \quad m_{v i s}$ for $\tau^{-} \rightarrow K^{-} \eta(\rightarrow \gamma \gamma) \nu_{\tau} \ldots \ldots \ldots \ldots \ldots \ldots$

$7.38 \quad m_{v i s}$ for $\tau^{-} \rightarrow K^{-} \eta\left(\rightarrow \pi^{0} \pi^{0} \pi^{0}\right) \nu_{\tau} \ldots \ldots \ldots \ldots \ldots$

$7.39 \quad m_{v i s}$ for $\tau^{-} \rightarrow K^{-} \eta\left(\rightarrow \pi^{+} \pi^{-} \pi^{0}\right) \nu_{\tau} \ldots \ldots \ldots \ldots \ldots$

$7.40 \quad m_{v i s}$ for $\tau^{-} \rightarrow K^{-} \eta\left(\rightarrow \pi^{+} \pi^{-} \gamma\right) \nu_{\tau} \ldots \ldots \ldots \ldots \ldots$

$7.41 \quad m_{v i s}$ for $\tau^{-} \rightarrow K^{-} \pi^{0} \eta(\rightarrow \gamma \gamma) \nu_{\tau} \ldots \ldots \ldots \ldots \ldots$

$7.42 \quad m_{v i s}$ for $\tau^{-} \rightarrow K^{-} \pi^{0} \eta\left(\rightarrow \pi^{0} \pi^{0} \pi^{0}\right) \nu_{\tau} \ldots \ldots \ldots \ldots$

$7.43 \quad m_{v i s}$ for $\tau^{-} \rightarrow K^{-} \pi^{0} \eta\left(\rightarrow \pi^{+} \pi^{-} \pi^{0}\right) \nu_{\tau} \ldots \ldots \ldots \ldots \ldots$

$7.44 \quad m_{v i s}$ for $\tau^{-} \rightarrow K^{-} \pi^{0} \eta\left(\rightarrow \pi^{+} \pi^{-} \gamma\right) \nu_{\tau} \ldots \ldots \ldots \ldots$

$7.45 \quad m_{v i s}$ for $\tau^{-} \rightarrow \pi^{-} K_{S}^{0} \eta(\rightarrow \gamma \gamma) \nu_{\tau} \ldots \ldots \ldots \ldots \ldots \ldots$

$7.46 \quad m_{v i s}$ for $\tau^{-} \rightarrow \pi^{-} K_{S}^{0} \eta\left(\rightarrow \pi^{0} \pi^{0} \pi^{0}\right) \nu_{\tau} \ldots \ldots \ldots \ldots \ldots 1$

$7.47 \quad m_{v i s}$ for $\tau^{-} \rightarrow \pi^{-} K_{S}^{0} \eta\left(\rightarrow \pi^{+} \pi^{-} \pi^{0}\right) \nu_{\tau} \ldots \ldots \ldots \ldots \ldots$

$7.48 \quad m_{v i s}$ for $\tau^{-} \rightarrow \pi^{-} K_{S}^{0} \eta\left(\rightarrow \pi^{+} \pi^{-} \gamma\right) \nu_{\tau} \ldots \ldots \ldots \ldots \ldots$

$7.49 \quad m_{v i s}$ for $\tau^{-} \rightarrow \pi^{-} K_{L}^{0} \eta(\rightarrow \gamma \gamma) \nu_{\tau} \ldots \ldots \ldots \ldots \ldots \ldots$

$7.50 \quad m_{v i s}$ for $\tau^{-} \rightarrow \pi^{-} K_{L}^{0} \eta\left(\rightarrow \pi^{0} \pi^{0} \pi^{0}\right) \nu_{\tau} \ldots \ldots \ldots \ldots \ldots$

$7.51 \quad m_{v i s}$ for $\tau^{-} \rightarrow \pi^{-} K_{L}^{0} \eta\left(\rightarrow \pi^{+} \pi^{-} \pi^{0}\right) \nu_{\tau} \ldots \ldots \ldots \ldots \ldots \ldots$

$7.52 \quad m_{v i s}$ for $\tau^{-} \rightarrow \pi^{-} K_{L}^{0} \eta\left(\rightarrow \pi^{+} \pi^{-} \gamma\right) \nu_{\tau} \ldots \ldots \ldots \ldots \ldots 2$

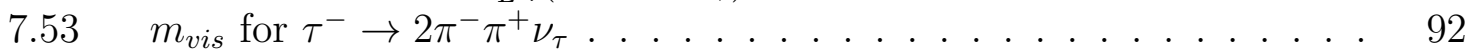

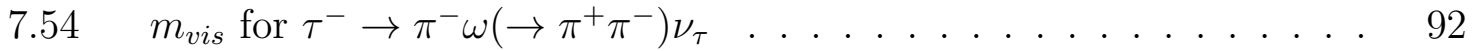

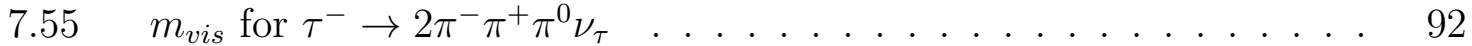

$7.56 \quad m_{v i s}$ for $\tau^{-} \rightarrow \pi^{-} \pi^{0} \omega\left(\rightarrow \pi^{+} \pi^{-}\right) \nu_{\tau} \ldots \ldots \ldots \ldots \ldots 2$

$7.57 m_{v i s}$ for $\tau^{-} \rightarrow 2 \pi^{-} \pi^{+} 2 \pi^{0} \nu_{\tau} \ldots \ldots \ldots \ldots \ldots \ldots$

$7.58 \quad m_{v i s}$ for $\tau^{-} \rightarrow \pi^{-} K^{-} K^{+} \nu_{\tau} \ldots \ldots \ldots \ldots \ldots \ldots$

$7.59 \quad m_{v i s}$ for $\tau^{-} \rightarrow \pi^{-} K^{-} K^{+} \pi^{0} \nu_{\tau} \ldots \ldots \ldots \ldots \ldots$

$7.60 \quad m_{v i s}$ for $\tau^{-} \rightarrow \pi^{-} \pi^{+} \pi^{-} K_{S}^{0} \nu_{\tau} \ldots \ldots \ldots \ldots \ldots \ldots$

$7.61 \quad m_{v i s}$ for $\tau^{-} \rightarrow \pi^{-} \pi^{+} \pi^{-} K_{L}^{0} \nu_{\tau} \ldots \ldots \ldots \ldots \ldots \ldots$

$7.62 \quad m_{v i s}$ for $\tau^{-} \rightarrow 2 \pi^{-} \pi^{+} \eta(\rightarrow \gamma \gamma) \nu_{\tau} \ldots \ldots \ldots \ldots \ldots \ldots$

$7.63 \quad m_{v i s}$ for $\tau^{-} \rightarrow 2 \pi^{-} \pi^{+} \eta\left(\rightarrow \pi^{0} \pi^{0} \pi^{0}\right) \nu_{\tau} \ldots \ldots \ldots \ldots \ldots$

$7.64 m_{v i s}$ for $\tau^{-} \rightarrow 2 \pi^{-} \pi^{+} \eta\left(\rightarrow \pi^{+} \pi^{-} \pi^{0}\right) \nu_{\tau} \ldots \ldots \ldots \ldots \ldots$

$7.65 m_{v i s}$ for $\tau^{-} \rightarrow 2 \pi^{-} \pi^{+} \eta\left(\rightarrow \pi^{+} \pi^{-} \gamma\right) \nu_{\tau} \ldots \ldots \ldots \ldots \ldots$

$7.66 \quad m_{v i s}$ for $\tau^{-} \rightarrow 2 \pi^{-} \pi^{+} \omega\left(\rightarrow \pi^{+} \pi^{-} \pi^{0}\right) \nu_{\tau} \ldots \ldots \ldots \ldots$

$7.67 \quad m_{v i s}$ for $\tau^{-} \rightarrow 2 \pi^{-} \pi^{+} \omega\left(\rightarrow \pi^{+} \pi^{-}\right) \nu_{\tau} \ldots \ldots \ldots \ldots \ldots$

$7.68 m_{v i s}$ for $\tau^{-} \rightarrow 2 \pi^{-} \pi^{+} \omega\left(\rightarrow \pi^{0} \gamma\right) \nu_{\tau} \ldots \ldots \ldots \ldots \ldots$

$7.69 \quad m_{v i s}$ for $\tau^{-} \rightarrow K^{-} \omega\left(\rightarrow \pi^{+} \pi^{-} \pi^{0}\right) \nu_{\tau} \ldots \ldots \ldots \ldots \ldots \ldots$

$7.70 \quad m_{v i s}$ for $\tau^{-} \rightarrow K^{-} \omega\left(\rightarrow \pi^{+} \pi^{-}\right) \nu_{\tau} \ldots \ldots \ldots \ldots \ldots$

$7.71 \quad m_{v i s}$ for $\tau^{-} \rightarrow K^{-} \omega\left(\rightarrow \pi^{0} \gamma\right) \nu_{\tau} \ldots \ldots \ldots \ldots \ldots \ldots$

$7.72 \quad m_{v i s}$ for $\tau^{-} \rightarrow K^{-} \phi\left(\rightarrow K^{+} K^{-}\right) \nu_{\tau} \ldots \ldots \ldots \ldots \ldots \ldots$

$7.73 \quad m_{v i s}$ for $\tau^{-} \rightarrow K^{-} \phi\left(\rightarrow K_{S}^{0} K_{L}^{0}\right) \nu_{\tau} \ldots \ldots \ldots \ldots \ldots$

$7.74 \quad m_{v i s}$ for $\tau^{-} \rightarrow K^{-} \pi^{-} \pi^{+} \nu_{\tau} \ldots \ldots \ldots \ldots \ldots$

$7.75 \quad m_{v i s}$ for $\tau^{-} \rightarrow K^{-} \pi^{-} \pi^{+} \pi^{0} \nu_{\tau} \ldots \ldots \ldots \ldots \ldots$

$7.76 \quad m_{v i s}$ for $\tau^{-} \rightarrow \pi^{-} 2 \pi^{0} \omega\left(\rightarrow \pi^{+} \pi^{-} \pi^{0}\right) \nu_{\tau} \ldots \ldots \ldots \ldots$ 
$7.77 \quad m_{v i s}$ for $\tau^{-} \rightarrow \pi^{-} 2 \pi^{0} \omega\left(\rightarrow \pi^{+} \pi^{-}\right) \nu_{\tau} \ldots \ldots \ldots \ldots \ldots$

$7.78 \quad m_{v i s}$ for $\tau^{-} \rightarrow \pi^{-} 2 \pi^{0} \omega\left(\rightarrow \pi^{0} \gamma\right) \nu_{\tau} \ldots \ldots \ldots \ldots \ldots$

$7.79 \quad m_{v i s}$ for $\tau^{-} \rightarrow 2 \pi^{-} \pi^{+} 3 \pi^{0} \nu_{\tau} \ldots \ldots \ldots \ldots \ldots \ldots$

$7.80 \quad m_{v i s}$ for $\tau^{-} \rightarrow 3 \pi^{-} 2 \pi^{+} \nu_{\tau} \ldots \ldots \ldots \ldots \ldots$

$7.81 \quad m_{v i s}$ for $\tau^{-} \rightarrow K^{-} 2 \pi^{-} 2 \pi^{+} \nu_{\tau} \ldots \ldots \ldots \ldots \ldots$

$7.82 \quad m_{v i s}$ for $\tau^{-} \rightarrow 3 \pi^{-} 2 \pi^{+} \pi^{0} \nu_{\tau} \ldots \ldots \ldots \ldots \ldots$

$7.83 \quad m_{v i s}$ for $\tau^{-} \rightarrow K^{-} 2 \pi^{-} 2 \pi^{+} \pi^{0} \nu_{\tau} \ldots \ldots \ldots \ldots \ldots 7$

$7.84 m_{v i s}$ for $\tau^{-} \rightarrow \pi^{-} f_{1}\left(\rightarrow 2 \pi^{-} 2 \pi^{+}\right) \nu_{\tau} \ldots \ldots \ldots \ldots \ldots$

$7.85 \quad m_{v i s}$ for $\tau^{-} \rightarrow \pi^{-} f_{1}\left(\rightarrow \pi^{-} \pi^{+} \eta\right) \nu_{\tau}$, where $\eta \rightarrow \gamma \gamma \ldots \ldots$. . . . 97

$7.86 \quad m_{v i s}$ for $\tau^{-} \rightarrow \pi^{-} f_{1}\left(\rightarrow \pi^{-} \pi^{+} \eta\right) \nu_{\tau}$, where $\eta \rightarrow 3 \pi^{0} \ldots \ldots \ldots 9$

$7.87 \quad m_{\text {vis }}$ for $\tau^{-} \rightarrow \pi^{-} f_{1}\left(\rightarrow \pi^{-} \pi^{+} \eta\right) \nu_{\tau}$, where $\eta \rightarrow \pi^{-} \pi^{+} \pi^{0} \ldots \ldots . .98$

$7.88 m_{\text {vis }}$ for $\tau^{-} \rightarrow \pi^{-} f_{1}\left(\rightarrow \pi^{-} \pi^{+} \eta\right) \nu_{\tau}$, where $\eta \rightarrow \pi^{-} \pi^{+} \gamma \ldots \ldots$

$7.89 \quad m_{v i s}$ for $\tau^{-} \rightarrow \pi^{-} 2 \pi^{0} \eta \nu_{\tau}$, where $\eta \rightarrow \gamma \gamma \ldots \ldots \ldots . \ldots 98$

$7.90 \quad m_{v i s}$ for $\tau^{-} \rightarrow \pi^{-} 2 \pi^{0} \eta \nu_{\tau}$, where $\eta \rightarrow 3 \pi^{0} \ldots \ldots \ldots$. . . . . 98

$7.91 \quad m_{v i s}$ for $\tau^{-} \rightarrow \pi^{-} 2 \pi^{0} \eta \nu_{\tau}$, where $\eta \rightarrow \pi^{-} \pi^{+} \pi^{0} \ldots \ldots \ldots 98$

$7.92 \quad m_{\text {vis }}$ for $\tau^{-} \rightarrow \pi^{-} 2 \pi^{0} \eta \nu_{\tau}$, where $\eta \rightarrow \pi^{-} \pi^{+} \gamma \ldots \ldots \ldots \ldots$

$8.1 \quad$ Final states in search for LFV decays $H \rightarrow \mu \tau \ldots \ldots$. . . . . . 100

$8.2 \quad$ Schematic description of a signal process . . . . . . . . . . . 107

8.3 Sketches for different SR and CR regions in CBA for $\ell \tau_{\text {had }} \ldots \ldots 118$

8.4 Distributions of fake factors in each signal region (SR1, SR2 and SR3) 124

8.5 Isolation factors for muons and electrons . . . . . . . . . . . . 125

8.6 Closure test in the preselection same sign region in $\mu \tau_{\text {had }}$ channel . 126

8.7 Closure test in the preselection same sign region in $e \tau_{\text {had }}$ channel . . 127

8.8 Distribution of $e \tau_{\text {had }}$ events as function of $\tau_{\text {had-vis }} p_{T}$ in the $Z \rightarrow e e$

(a) ID region (b) anti-ID region. . . . . . . . . . . . . . . 129

$8.9 \quad$ eFake estimate in $H \rightarrow e \tau_{\text {had }}$ SR1 region $\ldots \ldots \ldots \ldots \ldots$

8.10 eFake estimate in $H \rightarrow e \tau_{\text {had }}$ SR2 region . . . . . . . . . . . 131

8.11 eFake estimate in $H \rightarrow e \tau_{\text {had }}$ SR3 region . . . . . . . . . . 132

8.12 eFake estimate in same-sign $Z \rightarrow e e$ control region . . . . . . . . 134

8.13 eFake estimate in opposite-sign $Z \rightarrow e e$ control region . . . . . . . . 135

8.14 Distributions of $\mu \tau_{\text {had }}$ events in the $Z \rightarrow \mu \mu$ control region . . . . 136

8.15 Comparison of $e F F$ by fake factor method and by using MC $Z \rightarrow e e$

events . . . . . . . . . . . . . . . . . . . 140

8.16 Pre-fit distributions of representative kinematic quantities for different searches, channels and categories . . . . . . . . . . . 144

8.17 Pre-fit distributions of the Higgs boson mass for the $e \tau_{\text {had }}$ SR1-SR3 of the cut-based analysis . . . . . . . . . . . . . . . 145

8.18 Pre-fit distributions of the Higgs boson mass for the $\mu \tau_{\text {had }}$ SR1-SR3 of the cut-based analysis . . . . . . . . . . . . . . 145

8.19 The S+B fit ranking of different NPs in the combined MVA e $\tau$ channel149

8.20 The S+B fit ranking of different NPs in the combined MVA $\mu \tau$ channel150

8.21 Distributions of the BDT score after the background+signal fit in each signal region of the $e \tau$ search . . . . . . . . . . . . . . 151

8.22 Distributions of the BDT score after the background+signal fit in each signal region of the $\mu \tau$ search . . . . . . . . . . . . 152 
8.23 Candidate events of the $H \rightarrow e \tau$ search: $e \tau_{\mu}$ and $e \tau_{\text {had }}$. . . . . . . . 154

8.24 Candidate events of the $H \rightarrow \mu \tau$ search: $\mu \tau_{e}$ and $\mu \tau_{\text {had }} \ldots . . . . \quad 155$

8.25 Upper limits at 95\% CL on the LFV branching ratios of the Higgs

boson, $H \rightarrow e \tau$ and $H \rightarrow \mu \tau$. . . . . . . . . . . 156

8.26 Limits on the couplings $Y_{\tau \ell}$ and $Y_{\ell \tau} \ldots \ldots \ldots$. . . . . . . . 158 


\section{LIST OF TABLES}

\section{$\underline{\text { Table }}$}

2.1 Eigenvalues of the electromagnetic charge $Q$, the third component of the weak isospin $I^{3}$, and the weak hypercharge $Y=2 Q-2 I^{3}$ for the fermions of the SM. . . . . . . . . . . . . . . . . . 10

2.2 Cross sections for the major production processes assuming $m_{H}=$ $125 \mathrm{GeV}$........................ . . . 19

2.3 Branching ratios for various decay modes assuming $m_{H}=125 \mathrm{GeV} \quad 23$

3.1 Nominal design values of LHC operations parameters at ATLAS for 25 ns bunch crossing spacing . . . . . . . . . . . . . . . 28

$3.2 \quad$ Parameters of the Inner Detector . . . . . . . . . . . . . . . 32

3.3 Design energy resolution of the calorimeters . . . . . . . . . . 33

4.1 All algorithms for track reconstruction in the full detector with ITk layout ......................... 47

4.2 All algorithms for track reconstruction in the full detector with ID-

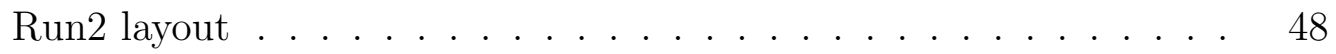

$4.3 \quad$ CPU requirements for ITk . . . . . . . . . . . . . . . . 52

$4.4 \quad$ CPU requiremnts for ID-Run2 . . . . . . . . . . . . . . . . 53

5.1 Summary of the detector components. . . . . . . . . . . . 57

$6.1 \quad \theta$ and $z$ discrimination for the BKLM and EKLM . . . . . . . . 65

$7.1 \tau$ branching fractions from PDG2020 version . . . . . . . . . . . 78

$7.2 \quad$ List of 92 initialized $\tau^{-}$decay modes with their BF. . . . . . . . . . 82

8.1 Generators used to describe the signal and background processes . . 103

8.2 BDT parameters used in the MVA analysis for $\ell \tau_{\ell^{\prime}}$ channels . . . . 112

8.3 BDT parameters used in the MVA analysis for $\ell \tau_{\text {had }}$ channels . . . . 112

$8.4 \quad$ MVA input variables importance $\ell \tau_{\text {had }}$ channels . . . . . . . . . . 113

8.5 BDT input variables used in the analysis . . . . . . . . . . . . 114

8.6 Baseline event selection and further categorization for the $\ell \tau_{\ell^{\prime}}$ and $\ell \tau_{\text {had }}$ channels . . . . . . . . . . . . . . . . 117

8.7 Definition of the categories of the $\ell \tau_{\text {had }}$ channel for the cut-based analysis . . . . . . . . . . . . . . . . . . 118

8.8 Summary of the event selection for the $\ell \tau_{\text {had }}$ QCD control regions for the fake factor method. . . . . . . . . . . . . . . . . . 119 
8.9 Summary of the event selection for the $\ell \tau_{\text {had }} \mathrm{W}+$ jets control regions for the fake factor method. . . . . . . . . . . . . . . . . . 119

8.10 Summary of the fake-estimate yields in the LFV $H \rightarrow \mu \tau_{\text {had }}$ signal region . . . . . . . . . . . . . . . . . . 139

8.11 Summary of the fake-estimate yields in the LFV $H \rightarrow e \tau_{\text {had }}$ signal regions . . . . . . . . . . . . . . . . . . 139

8.12 Summary of the $e \rightarrow \tau_{\text {had }}$ estimate yields in the LFV $H \rightarrow e \tau_{\text {had }}$ signal region . . . . . . . . . . . . . . . . . . 141

8.13 Summary of individual sets of systematic uncertainties . . . . . . 148

8.14 Event yields and predictions as determined by the background-only fit in different signal regions of the $H \rightarrow e \tau$ analysis . . . . . . . 153

8.15 Event yields and predictions as determined by the background-only fit in different signal regions of the $H \rightarrow \mu \tau$ analysis . . . . . . 153 


\section{CHAPTER I}

\section{OUTLINE OF THE THESIS}

Searches for new physics using lepton flavor violating signatures in the decays of the Higgs boson into final states containing the $\tau$ lepton using data collected in proton-proton collisions by the ATLAS experiment at a center-of-mass energy of 13 $\mathrm{TeV}$ are presented. Also, modelling of Standard Model decays of the tau lepton at the Belle II experiment are presented.

The thesis is organized in the following manner, Chapter 1 being this introduction.

In Chapter 2, some of the theoretical backgrounds on the Standard Model of particle physics building up to the properties of the Higgs boson are presented.

In Chapter 3, the experimental setup of the ATLAS detector at the Large Hadron Collider is discussed, where the searches for Higgs decays are performed.

In Chapter 4, the technical contributions to the service task are discussed, based on which I obtained the authorship qualification in the ATLAS collaboration. These tasks include benchmarking of the CPU requirements for the track reconstruction for the Phase II upgrade of the Inner Tracker of the ATLAS experiment.

In Chapter 5, the Belle II experimental setup for understanding the decays of the tau lepton at the SuperKEKB collider is presented.

In Chapter 6, the techincal contributions on data quality monitoring of the muon detector of the Belle II experiment are mentioned, based on which I obtained the 
authorship qualification in the Belle II collaboration.

In Chapter 7, the physics analysis on modelling of the Standard Model decays of the tau lepton at the Belle II experiment are presented.

In Chapter 8, the physics analysis on search of lepton flavor violating decays of the Higgs boson at the ATLAS expriment are presented. 


\section{CHAPTER II}

\section{STANDARD MODEL OF PARTICLE PHYSICS}

This chapter motivates and lays the groundwork for the addition of the Higgs boson to the Standard Model (SM) of particle physics. This requires an understanding of gauge theories, which are intimately related to the notion of symmetry. The implications of the invariance of the laws of physics under transformations are first discussed in section 2.1. Following a discussion on the work of Dirac that describes the Lagrangian of free fermions in section 2.2, the addition of a gauge field to the Lagrangian satisfying invariance under $\mathrm{U}(1)_{Y}$ transformations leads to unification of Quantum Mechanics (QM) with Maxwell's laws of electromagnetism are discussed in section 2.3. This unification is extended in Section 2.4 to combine the electromagnetic and weak forces into the electroweak force, which lays the foundation of the Higgs mechanism as discussed in section 2.5. Although the strong interaction is not directly related to the Higgs mechanism, an understanding of Quantum Chromodynamics (QCD) forms an integral part of the SM and is discussed in section 2.6. In the next section (2.7), the production and decays of the Higgs relevant to the LHC are discussed. Final section 2.8 describes the lepton flavor violating decays of the Higgs boson as an unambiguous search for new physics, in an attempt to describe Nature beyond the SM of particle physics. 


\subsection{Noether's Theorem}

The fundamental notion of symmetry in nature is the underlying theory of particles, and physics in general. In 1918 Emmy Noether proved a theorem (known as Noether's Theorem) that for every transformation of a field $\phi(x)$ which leaves the action invariant there exists a conserved current [1]. The invariance of the action is equivalent to invariance of the Lagrangian density $\mathcal{L}(x)$, which is a function of both $\phi(x)$ and its derivative $\partial^{\mu} \phi(x)$. The action is defined by the integral of the Lagrangian density (henceforth referred to simply as the Lagrangian) over all space-time.

$$
S=\int \mathcal{L}(x) d^{4} x
$$

Let us consider an infinitesimal transformation of the field $\phi(x) \rightarrow \phi(x)+\delta \phi(x)$ that conserves the action:

$$
\begin{aligned}
0 & =\delta S \\
& =\int d^{4} x\left\{\frac{\partial \mathcal{L}}{\partial \phi} \delta \phi+\frac{\partial \mathcal{L}}{\partial\left(\partial_{\mu} \phi\right)} \delta\left(\partial_{\mu} \phi\right)\right\} \\
& =\int d^{4} x\left\{\frac{\partial \mathcal{L}}{\partial \phi} \delta \phi-\partial_{\mu}\left(\frac{\partial \mathcal{L}}{\partial\left(\partial_{\mu} \phi\right)}\right) \delta \phi+\partial_{\mu}\left(\frac{\partial \mathcal{L}}{\partial\left(\partial_{\mu} \phi\right)} \delta \phi\right)\right\}
\end{aligned}
$$

The third term evaluates to zero assuming no boundary terms in the action. This indicates that invariance of the action is equivalent to the Euler-Lagrange equation of motion:

$$
\frac{\partial \mathcal{L}}{\partial \phi}-\partial_{\mu}\left(\frac{\partial \mathcal{L}}{\partial\left(\partial_{\mu} \phi\right)}\right)=0
$$

One can thus require that a shift in the Lagrangian induced by a local transformation of the field must be zero up to a local four divergence $\left(\partial_{\mu} \mathcal{J}^{\mu}(x)\right)$ :

$$
\mathcal{L}(x) \rightarrow \mathcal{L}^{\prime}(x)=\mathcal{L}(x)+\partial_{\mu} \mathcal{J}^{\mu}(x) \Rightarrow \delta \mathcal{L}(x)=\partial_{\mu} \mathcal{J}^{\mu}(x)
$$


The shift in the Lagrangian can also be written with respect to the field:

$$
\begin{aligned}
\delta \mathcal{L}(x) & =\frac{\partial \mathcal{L}}{\partial \phi} \delta \phi+\frac{\partial \mathcal{L}}{\partial\left(\partial_{\mu} \phi\right)} \delta\left(\partial_{\mu} \phi\right) \\
& =\frac{\partial \mathcal{L}}{\partial \phi} \delta \phi-\partial_{\mu}\left(\frac{\partial \mathcal{L}}{\partial\left(\partial_{\mu} \phi\right)}\right) \delta \phi+\partial_{\mu}\left(\frac{\partial \mathcal{L}}{\partial\left(\partial_{\mu} \phi\right)} \delta \phi\right) \\
& =\partial_{\mu}\left(\frac{\partial \mathcal{L}}{\partial\left(\partial_{\mu} \phi\right)} \delta \phi\right)
\end{aligned}
$$

Equating the two forms of $\delta \mathcal{L}(x)$, one obtains a conserved current $j^{\mu}(x)$ :

$$
\begin{aligned}
j^{\mu}(x) & \equiv \frac{\partial \mathcal{L}(x)}{\partial\left(\partial_{\mu} \phi\right)} \delta \phi+\mathcal{J}^{\mu}(x) \\
\partial_{\mu} j^{\mu}(x) & =\partial_{\mu}\left(\frac{\partial \mathcal{L}(x)}{\partial\left(\partial_{\mu} \phi\right)} \delta \phi\right)+\partial_{\mu} \mathcal{J}^{\mu}(x)=0
\end{aligned}
$$

\subsection{Dirac Lagrangian}

The behavior of relativistic spin-1/2 fields $\psi(x)$ in free space is described by the Dirac Lagrangian [2]:

$$
\mathcal{L}_{\text {Dirac }}(x)=\bar{\psi}(x)\left(i \gamma^{\mu} \partial_{\mu}-m\right) \psi(x)
$$

In 4 -dimensions the terms $\gamma^{\mu}$ are $4 \times 4$ matrices satisfying the anti-commutation relationship:

$$
\left\{\gamma^{\mu}, \gamma^{\nu}\right\}=2 g^{\mu \nu}
$$

where $g^{\mu \nu}$ is the metric tensor. In the four dimensional Minkowski space, these matrices can be represented in terms of the $2 \times 2$ Pauli sigma matrices $\sigma^{i}, i=1,2,3$ :

$$
\gamma^{\mu}=\left(\begin{array}{cc}
0 & \sigma^{\mu} \\
\bar{\sigma}^{\mu} & 0
\end{array}\right)
$$

$\sigma^{\mu}$ and $\bar{\sigma}^{\mu}$ are the four-vector notations for the Pauli sigma matrices, such that $\sigma^{\mu} \equiv(\mathbf{I}, \sigma)$ and $\bar{\sigma}^{\mu} \equiv(\mathbf{I},-\sigma)$, with $\sigma \equiv\left(\sigma^{1}, \sigma^{2}, \sigma^{3}\right)$. The term $\bar{\psi}(x) \equiv \psi^{\dagger} \gamma^{0}$ requires 
the additional $\gamma^{0}$ for the $\bar{\psi} \psi$ term to be Lorentz invariant. The term $\psi(x)$ can be further decomposed into left and right handed Weyl spinors:

$$
\psi=\left(\begin{array}{c}
\psi_{L} \\
\psi_{R}
\end{array}\right)
$$

The Dirac equation of motion for $\psi(x)$ is obtained by applying the Euler-Lagrange formula to $\mathcal{L}_{\text {Dirac }}(x)$ :

$$
\left(i \gamma^{\mu} \partial_{\mu}-m\right) \psi(x)=0
$$

This represents the equation of motion for a free (non-interacting) fermion. The next section shows how interactions with the electromagnetic field can be incorporated into the Dirac Lagrangian.

\subsection{Quantum Electrodynamics}

At low energies up to the order of a few GeV, Quantum Electrodynamics (QED) represents a unification of QM with Maxwell's laws of electromagnetism that describe how charged fermions interact with the electromagnetic fields where the effects from heavy gauge bosons $W^{ \pm} / Z^{0}$ are negligeble:

$$
\begin{aligned}
\nabla \cdot \mathbf{E} & =\frac{\rho}{\varepsilon_{0}} \\
\nabla \cdot \mathbf{B} & =0 \\
\nabla \times \mathbf{E} & =-\frac{\partial \mathbf{B}}{\partial t} \\
\nabla \times \mathbf{B} & =\mu_{0} \mathbf{J}+\mu_{0} \varepsilon_{0} \frac{\partial \mathbf{E}}{\partial t}
\end{aligned}
$$

$\mathbf{E}$ is the electric field, $\mathbf{B}$ is the magnetic field, $\rho$ is the electric charge density of space, $\mu_{0}$ is the permeability of free space, and $\varepsilon_{0}$ is the permittivity of free space.

Switching to natural units and introducing the electromagnetic four-potential $A^{\mu}=(\phi, \mathbf{A})$, where $\mathbf{E}=-\frac{\partial \mathbf{A}}{\partial t}-\nabla \phi$ and $\mathbf{B}=\nabla \times \mathbf{A}$, and a corresponding field 
strength tensor $F^{\mu \nu}=\partial^{\mu} A^{\nu}-\partial^{\nu} A^{\mu}$, the Lagrangian for QED is given as:

$$
\mathcal{L}_{\mathrm{QED}}=\bar{\psi}\left(i \gamma^{\mu} D_{\mu}-m\right) \psi-\frac{1}{4} F_{\mu \nu} F^{\mu \nu}
$$

where $D_{\mu} \equiv \partial_{\mu}-i e A_{\mu}$ is the covariant derivative along the tangent vector of the manifold as required for gauge invariance, and $e=|e|$ is taken to be positive. This term ultimately yields the interaction terms in the Lagrangian between the fermion and electromagnetic field. One recovers Maxwell's equations after applying the EulerLagrange equation with respect to $A^{\mu}$ :

$$
\begin{aligned}
\mathcal{L}_{Q E D} & =\bar{\psi}\left(i \gamma^{\mu} D_{\mu}-m\right) \psi-\frac{1}{4}\left(\partial_{\mu} A_{\nu}-\partial_{\nu} A_{\mu}\right)\left(\partial^{\mu} A^{\nu}-\partial^{\nu} A^{\mu}\right) \\
& =\bar{\psi}\left(i \gamma^{\mu} D_{\mu}-m\right) \psi-\frac{1}{4}\left(\partial_{\mu} A_{\nu} \partial^{\mu} A^{\nu}-\partial_{\mu} A_{\nu} \partial^{\nu} A^{\mu}-\partial_{\nu} A_{\mu} \partial^{\mu} A^{\nu}+\partial_{\nu} A_{\mu} \partial^{\nu} A^{\mu}\right) \\
& =\bar{\psi}\left(i \gamma^{\mu} \partial_{\mu}-e \gamma^{\mu} A_{\mu}-m\right) \psi-\frac{1}{2}\left(\partial_{\mu} A_{\nu} \partial^{\mu} A^{\nu}-\partial_{\mu} A_{\nu} \partial^{\nu} A^{\mu}\right)
\end{aligned}
$$

$$
\begin{aligned}
& \frac{\partial \mathcal{L}}{\partial A_{\mu}}-\partial_{\nu}\left(\frac{\partial \mathcal{L}}{\partial\left(\partial_{\nu} A_{\mu}\right)}\right) \quad=0 \\
& \Rightarrow \partial_{\nu}\left(\partial^{\mu} A^{\nu}-\partial^{\nu} A^{\mu}\right)=-e \bar{\psi} \gamma^{\mu} \psi
\end{aligned}
$$

Defining the four current $j^{\mu} \equiv-e \bar{\psi} \gamma^{\mu} \psi$, the last equation can be written in a more elegant form:

$$
\partial_{\nu} F^{\mu \nu}=j^{\mu}
$$

The conservation of $j^{\mu}$ follows directly:

$$
\begin{aligned}
\partial_{\mu} j^{\mu} & =\partial_{\mu} \partial_{\nu} F^{\mu \nu} \\
& =\partial_{\mu} \partial_{\nu}\left(\partial^{\mu} A^{\nu}-\partial^{\nu} A^{\mu}\right) \\
& =0
\end{aligned}
$$

Eq. 2.16 yields four differential equations, one for each index of $\mu$. Writing out the $\mu=0$ and $\mu=1,2,3$ components separately and writing $j^{\mu}=(\rho, \mathbf{J})$ in terms of its 
temporal and spatial components $\rho$ and $\mathbf{J}$, respectively, one obtains two of Maxwell's four equations:

$$
\begin{array}{ll}
-\nabla \cdot \frac{\partial \mathbf{A}}{\partial t}+\nabla^{2} \phi & =j^{0} \Leftrightarrow \nabla \cdot \mathbf{E}=\frac{\rho}{\varepsilon_{0}} \\
\nabla \times(\nabla \times \mathbf{A})-\frac{\partial^{2} \mathbf{A}}{\partial t^{2}}+\frac{\partial}{\partial t} \nabla \phi & =j^{i} \Leftrightarrow \nabla \times \mathbf{B}=\mu_{0} \mathbf{J}+\mu_{0} \varepsilon_{0} \frac{\partial \mathbf{E}}{\partial t}
\end{array}
$$

Noting that $\mathbf{E}$ and $\mathbf{B}$ are functions of $\mathbf{A}$ and $\phi$, the last two equations follow directly from the identities of second derivatives:

$$
\begin{aligned}
& \nabla \times \mathbf{E}=-\frac{\partial}{\partial t} \nabla \times \mathbf{A}-\nabla \times \nabla \phi=-\frac{\partial \mathbf{B}}{\partial t} \\
& \nabla \cdot \mathbf{B}=\nabla \cdot(\nabla \times \mathbf{A}) \quad=0
\end{aligned}
$$

Applying the Euler-Lagrange equation with respect to $\psi$, one obtains the equation of motion of fermions. With respect to Eq. 2.11, the only additional term is the interaction term with the field $A_{\mu}$ :

$$
\left(i \gamma^{\mu} \partial_{\mu}-m\right) \psi=-e \gamma^{\mu} A_{\mu} \psi
$$

$\mathcal{L}_{\mathrm{QED}}$ is invariant under a gauge transformation of the field $\psi$ when the gauge field $A_{\mu}$ is simultaneously transformed in the following way:

$$
\begin{aligned}
& \psi(x) \rightarrow \psi^{\prime}(x)=\psi(x) e^{i \theta(x)} \\
& A_{\mu}(x) \rightarrow A_{\mu}^{\prime}(x)=A_{\mu}(x)+\frac{1}{e} \partial_{\mu} \theta(x)
\end{aligned}
$$

The terms $F^{\mu \nu}$ (and therefore $F^{\mu \nu} F_{\mu \nu}$ ) and $m \bar{\psi} \psi$ are manifestedly invariant. The interaction and kinetic terms each pick up a term differing by a sign and cancel:

$$
\begin{aligned}
\bar{\psi} \gamma^{\mu} D_{\mu} \psi \rightarrow \bar{\psi}^{\prime} \gamma^{\mu} D_{\mu}^{\prime} \psi^{\prime} & =\bar{\psi} e^{-i \theta(x)} \gamma^{\mu}\left(\partial_{\mu}-i e A_{\mu}(x)-i \partial_{\mu} \theta(x)\right) \psi e^{i \theta(x)} \\
& =\bar{\psi} \gamma^{\mu} D_{\mu} \psi+\bar{\psi} i \gamma^{\mu} \partial_{\mu} \theta(x) \psi-\bar{\psi} i \gamma^{\mu} \psi \partial_{\mu} \theta(x) \\
& =\bar{\psi} \gamma^{\mu} D_{\mu} \psi
\end{aligned}
$$




\subsection{Electroweak Unification}

While QED works well in describing the interactions between charged fermions and the electromagnetic field, it does not describe phenomena arising from weak interactions [3], such as radioactive $\beta$ decays. To incorporate a description of radiactivity, we need to extend the symmetry of the model to a larger symmetry group $[4,5,6]$.

The spin-1 gauge fields $W_{\mu}^{a}(a=1,2,3)$ transform under the adjoint of the $\mathrm{SU}(2)_{L}$ group with associated generators $T^{a}=\frac{1}{2} \sigma^{a}$, where $\sigma^{a}$ are the Pauli matrices. The gauge field $B_{\mu}$ is associated with $\mathrm{U}(1)_{Y}$.

Fermions enter as left-handed doublets $\Psi_{L}$ of $\mathrm{SU}(2)_{L}$, while the right handed fermions $\psi_{R}$ are $\mathrm{SU}(2)_{L}$ singlets that are charged under $\mathrm{U}(1)_{Y}$. Here, $\Psi_{L}$ is a doublet representation of $\mathrm{SU}(2)_{L}$, and $\psi_{R}$ is a singlet under $\mathrm{SU}(2)_{L}$, but transforms under $\mathrm{U}(1)_{Y}$ :

The Electroweak Lagrangian invariant under $\mathrm{SU}(2)_{L} \times \mathrm{U}(1)_{Y}$ can be written as:

$$
\mathcal{L}_{E W}=\bar{\Psi}_{L} i \gamma^{\mu} D_{\mu}^{L} \Psi_{L}+\bar{\psi}_{R} i \gamma^{\mu} D_{\mu}^{R} \psi_{R}-\frac{1}{4} W_{a}^{\mu \nu} W_{\mu \nu}^{a}-\frac{1}{4} B^{\mu \nu} B_{\mu \nu}
$$

where

$$
\Psi_{L}=\left(\begin{array}{c}
\psi_{L}^{\prime} \\
\psi_{L}
\end{array}\right)
$$

Here, $\Psi_{L}$ and $\psi_{R}$ can be either leptons or quarks. There are three generations of both, which will remain implicit in the notation, shown explicitly only for the first 


\begin{tabular}{c|ccccccc}
\hline \hline & $\nu_{L}$ & $e_{L}$ & $e_{R}$ & $u_{L}$ & $d_{L}$ & $u_{R}$ & $d_{R}$ \\
\hline$Q$ & 0 & -1 & -1 & $+2 / 3$ & $-1 / 3$ & $+2 / 3$ & $-1 / 3$ \\
$I^{3}$ & $+1 / 2$ & $-1 / 2$ & 0 & $+1 / 2$ & $-1 / 2$ & 0 & 0 \\
\hline$Y$ & -1 & -1 & -2 & $+1 / 3$ & $+1 / 3$ & $+4 / 3$ & $-2 / 3$ \\
\hline
\end{tabular}

Table 2.1: Eigenvalues of the electromagnetic charge $Q$, the third component of the weak isospin $I^{3}$, and the weak hypercharge $Y=2 Q-2 I^{3}$ for the fermions of the SM.

generation for example:

$$
\begin{gathered}
\text { Leptons : } \\
\left(\begin{array}{c}
\psi_{L}^{\prime} \\
\psi_{L}
\end{array}\right)=\left(\begin{array}{c}
\nu_{L} \\
e_{L}
\end{array}\right) ;\left(\begin{array}{c}
\psi_{L}^{\prime} \\
\psi_{L}
\end{array}\right)=\left(\begin{array}{c}
u_{L} \\
d_{L}
\end{array}\right) \\
\psi_{R}=e_{R} ; \psi_{R}=u_{R}, d_{R}
\end{gathered}
$$

The covariant derivative contains the four gauge fields that will form the interaction terms with the fermions. This is different for the left-handed doublets and right-handed singlets:

$$
\begin{aligned}
D_{\mu}^{L} & =\partial_{\mu}-i g_{1} \frac{Y}{2} B_{\mu}-i g_{2} T^{a} W_{\mu}^{a} \\
D_{\mu}^{R} & =\partial_{\mu}-i g_{1} \frac{Y}{2} B_{\mu}
\end{aligned}
$$

The hypercharge of the fermions is $Y=2 Q-2 I^{3}$, where $Q$ is the electromagnetic charge and $I^{3}$ is the third component of the weak isospin. The eigenvalues of each for the different fermion flavors in the first generation (taken just as an example) are listed in Table 2.1.

Here, $g_{1}$ and $g_{2}$ are the coupling constants of the fermions to the gauge fields. The form of the field strength tensor $B_{\mu \nu}$ of $B_{\mu}$ is identical to that of the photon in QED. $W_{\mu \nu}^{a}$ is the field strength tensor of $W_{\mu}^{a}$. Because the generators $T^{a}$ of $\mathrm{SU}(2)_{L}$ do not 
commute, $W_{\mu \nu}^{a}$ picks up an additional term:

$$
\begin{aligned}
W_{\mu \nu}^{a} & =\partial_{\mu} W_{\nu}^{a}-\partial_{\nu} W_{\mu}^{a}+g_{2} \epsilon^{a b c} W_{\mu}^{b} W_{\nu}^{c} \\
B_{\mu \nu} & =\partial_{\mu} B_{\nu}-\partial_{\nu} B_{\mu}
\end{aligned}
$$

$\epsilon^{a b c}$ is the antisymmetric tensor that arises due to the commutation relation that is the $\mathrm{SU}(2)_{L}$ algebra. There is no such term in $B_{\mu \nu}$ since the lone $\mathrm{U}(1)_{Y}$ generator Y trivially commutes:

$$
\begin{aligned}
& {\left[T^{a}, T^{b}\right]=i \epsilon^{a b c} T^{c}} \\
& {[Y, Y]=0}
\end{aligned}
$$

The extra term in $W_{\mu \nu}^{a}$ from the non-abelian nature of $\mathrm{SU}(2)_{L}$ gives rise to selfinteractions among the gauge bosons in this group that aren't present in $\mathrm{U}(1)_{Y}[7]$.

An $\mathrm{SU}(2)_{L}$ transformation of the Lagrangian yields the transformed fermion doublet and gauge fields required for invariance of the Lagrangian:

$$
\begin{aligned}
\Psi_{L}^{\prime} & =\Psi_{L} e^{i \beta_{a}(x) T^{a}} \\
\vec{W}_{\mu}^{\prime} & =\vec{W}_{\mu}-\frac{1}{g_{2}} \partial_{\mu} \vec{\beta}(x)-\vec{\beta}(x) \times \vec{W}_{\mu}
\end{aligned}
$$

However, the mass terms breaks this symmetry. Expanding the fermion field in terms of the left and right handed components illustrates the reason:

$$
\begin{aligned}
m \bar{\Psi} \Psi & =m \bar{\Psi}\left(\frac{1}{2}\left(1-\gamma^{5}\right)+\frac{1}{2}\left(1+\gamma^{5}\right)\right) \Psi \\
& =m\left(\bar{\Psi}_{R} \Psi_{L}+\bar{\Psi}_{L} \Psi_{R}\right)
\end{aligned}
$$

Since only $\Psi_{L}$ transforms under $\mathrm{SU}(2)_{L}$, these mass terms are not invariant under the transformation in Equation 2.29; neither are mass terms $M_{a}^{2} W_{\mu}^{a} W^{a \mu}$ for the bosons. Fermions and bosons do acquire mass via the Higgs mechanism desribed in Section 2.5.

In general, the physical bosons that couple with the fermions can be mixtures of 
the gauge bosons. The mixing of the $W_{\mu}^{3}$ and the $B_{\mu}$ can be represented by a rotation by the Weinberg angle $\theta_{W}$.

Using the notations:

$$
\sin \theta_{W} \equiv s_{W} \equiv \frac{g_{1}}{\sqrt{g_{1}^{2}+g_{2}^{2}}}
$$

and

$$
\cos \theta_{W} \equiv c_{W} \equiv \frac{g_{2}}{\sqrt{g_{1}^{2}+g_{2}^{2}}}
$$

the rotation can be written in the following way:

$$
\left(\begin{array}{c}
A_{\mu} \\
Z_{\mu}
\end{array}\right)=\left(\begin{array}{cc}
c_{W} & s_{W} \\
-s_{W} & c_{W}
\end{array}\right)\left(\begin{array}{c}
B_{\mu} \\
W_{\mu}^{3}
\end{array}\right)
$$

Thus, $B_{\mu}$ and $W_{\mu}^{3}$ can be written in terms of $A_{\mu}, Z_{\mu}$, and $\theta_{W}$ :

$$
\begin{aligned}
B_{\mu} & =c_{W} A_{\mu}-s_{W} Z_{\mu} \\
W_{\mu}^{3} & =s_{W} A_{\mu}+c_{W} Z_{\mu}
\end{aligned}
$$

The covariant derivative can be written in terms of the mass eigenstates $A_{\mu}, Z_{\mu}$, and $W_{\mu}^{ \pm}$corresponding to the physical bosons $\gamma, \mathrm{Z}$, and $W^{ \pm}$, respectively in the following manner: 


$$
\begin{aligned}
i D_{\mu}^{L} & =i\left(\partial_{\mu}-i g_{1} \frac{Y}{2} B_{\mu}-i g_{2} T^{a} W_{\mu}^{a}\right) \\
& =\left(\begin{array}{cc}
a_{\mu}^{11} & a_{\mu}^{12} \\
a_{\mu}^{21} & a_{\mu}^{22}
\end{array}\right), \\
a_{\mu}^{11} & =i \partial_{\mu}+\frac{1}{2} g_{1} Y B_{\mu}+\frac{1}{2} g_{2} W_{\mu}^{3} \\
& =i \partial_{\mu}+\frac{1}{2}\left(g_{1} c_{W} Y+g_{2} s_{W}\right) A_{\mu}-\frac{1}{2}\left(g_{1} s_{W} Y-g_{2} c_{W}\right) Z_{\mu} \\
a_{\mu}^{12} & =\frac{1}{2} g_{2}\left(W_{\mu}^{1}-i W_{\mu}^{2}\right) \equiv \frac{1}{\sqrt{2}} g_{2} W_{\mu}^{+} \\
a_{\mu}^{21} & =\frac{1}{2} g_{2}\left(W_{\mu}^{1}+i W_{\mu}^{2}\right) \equiv \frac{1}{\sqrt{2}} g_{2} W_{\mu}^{-} \\
a_{\mu}^{22} & =i \partial_{\mu}+\frac{1}{2} g_{1} Y B_{\mu}-\frac{1}{2} g_{2} W_{\mu}^{3} \\
& =i \partial_{\mu}+\frac{1}{2}\left(g_{1} c_{W} Y-g_{2} s_{W}\right) A_{\mu}-\frac{1}{2}\left(g_{1} s_{W} Y+g_{2} c_{W}\right) Z_{\mu}
\end{aligned}
$$

Multiplying the interaction terms with the fermion doublets and rewriting the coefficients in terms of $\theta_{W}$ reveals the electroweak fermion-boson interactions:

$$
\begin{aligned}
\bar{\Psi}_{L} i \gamma^{\mu} D_{\mu}^{L} \Psi_{L}= & \left(\begin{array}{cc}
\bar{\psi}_{L}^{\prime} & \bar{\psi}_{L}
\end{array}\right) \gamma^{\mu}\left(\begin{array}{cc}
a_{\mu}^{11} & a_{\mu}^{12} \\
a_{\mu}^{21} & a_{\mu}^{22}
\end{array}\right)\left(\begin{array}{c}
\psi_{L}^{\prime} \\
\psi_{L}
\end{array}\right) \\
= & \bar{\psi}_{L}^{\prime} \gamma^{\mu} a_{\mu}^{11} \psi_{L}^{\prime}+\bar{\psi}_{L}^{\prime} \gamma^{\mu} a_{\mu}^{12} \psi_{L} \\
& +\bar{\psi}_{L} \gamma^{\mu} a_{\mu}^{21} \psi_{L}^{\prime}+\bar{\psi}_{L} \gamma^{\mu} a_{\mu}^{22} \psi_{L} \\
= & i \bar{\psi}_{L}^{\prime} \gamma^{\mu} \partial_{\mu} \psi_{L}^{\prime}+i \bar{\psi}_{L} \gamma^{\mu} \partial_{\mu} \psi_{L} \\
& +\frac{1}{2} e(Y+1) \bar{\psi}_{L}^{\prime} \gamma^{\mu} \psi_{L}^{\prime} A_{\mu}+\frac{1}{2} e(Y-1) \bar{\psi}_{L} \gamma^{\mu} \psi_{L} A_{\mu} \\
& -\frac{1}{2}\left(g_{1} s_{W} Y-g_{2} c_{W}\right) \bar{\psi}_{L}^{\prime} \gamma^{\mu} \psi_{L}^{\prime} Z_{\mu}-\frac{1}{2}\left(g_{1} s_{W} Y+g_{2} c_{W}\right) \bar{\psi}_{L} \gamma^{\mu} \psi_{L} Z_{\mu} \\
& +\frac{1}{\sqrt{2}} g_{2} \bar{\psi}_{L}^{\prime} \gamma^{\mu} \psi_{L} W_{\mu}^{+}+\frac{1}{\sqrt{2}} g_{2} \bar{\psi}_{L} \gamma^{\mu} \psi_{L}^{\prime} W_{\mu}^{-}
\end{aligned}
$$

The identity $g_{1} c_{W}=g_{2} s_{W} \equiv e$, the electromagnetic charge, has been used in the $A_{\mu}$ terms. The right handed singlet terms are similar:

$$
\begin{aligned}
\bar{\psi}_{R} i \gamma^{\mu} D_{\mu}^{R} \psi_{R} & =\bar{\psi}_{R} i \gamma_{\mu}\left(\partial_{\mu}-i g_{1} \frac{Y}{2} B_{\mu}\right) \psi_{R} \\
& =i \bar{\psi}_{R} \gamma_{\mu} \partial_{\mu} \psi_{R}+\frac{1}{2} e Y \bar{\psi}_{R} \gamma_{\mu} \psi_{R} A_{\mu}-\frac{1}{2} g_{1} s_{W} Y \bar{\psi}_{R} \gamma_{\mu} \psi_{R} Z_{\mu}
\end{aligned}
$$


Inserting the SM fermions in place of $\psi_{L}^{\prime}, \psi_{L}$, and $\psi_{R}$ and replacing the weak hypercharge with its corresponding eigenvalues, one gets the seventeen interaction terms as listed below:

Left - Handed Interactions :

\begin{tabular}{|c|c|c|}
\hline EM & $\begin{array}{c}- \\
\frac{2}{3} e \bar{u}_{L} \gamma^{\mu} u_{L} A_{\mu}\end{array}$ & $\begin{array}{l}-e \bar{e}_{L} \gamma^{\mu} e_{L} A_{\mu} \\
\frac{1}{6} e \bar{d}_{L} \gamma^{\mu} d_{L} A_{\mu}\end{array}$ \\
\hline Neutral Current & $\begin{array}{c}\frac{1}{2}\left(g_{1} s_{W}+g_{2} c_{W}\right) \bar{\nu}_{L}^{\prime} \gamma^{\mu} \nu_{L}^{\prime} Z_{\mu} \\
-\frac{1}{2}\left(\frac{1}{3} g_{1} s_{W}-g_{2} c_{W}\right) \bar{u}_{L} \gamma^{\mu} u_{L} Z_{\mu}\end{array}$ & $\begin{array}{c}\frac{1}{2}\left(g_{1} s_{W}-g_{2} c_{W}\right) \bar{e}_{L} \gamma^{\mu} e_{L} Z_{\mu} \\
-\frac{1}{2}\left(\frac{1}{3} g_{1} s_{W}+g_{2} c_{W}\right) \bar{d}_{L} \gamma^{\mu} d_{L} Z_{\mu}\end{array}$ \\
\hline Charged Current & $\begin{array}{l}\frac{1}{\sqrt{2}} g_{2} \bar{\nu}_{L} \gamma^{\mu} e_{L} W_{\mu}^{+} \\
\frac{1}{\sqrt{2}} g_{2} \bar{u}_{L} \gamma^{\mu} d_{L} W_{\mu}^{+}\end{array}$ & $\begin{array}{l}\frac{1}{\sqrt{2}} g_{2} \bar{e}_{L} \gamma^{\mu} \nu_{L} W_{\mu}^{-} \\
\frac{1}{\sqrt{2}} g_{2} \bar{d}_{L} \gamma^{\mu} u_{L} W_{\mu}^{-}\end{array}$ \\
\hline \multicolumn{3}{|c|}{ Right - Handed Interactions } \\
\hline EM & $\begin{array}{c}- \\
\frac{2}{3} e \bar{u}_{R} \gamma_{\mu} u_{R} A_{\mu}\end{array}$ & $\begin{array}{l}-e \bar{e}_{R} \gamma_{\mu} e_{R} A_{\mu} \\
-\frac{1}{3} e \bar{d}_{R} \gamma_{\mu} d_{R} A_{\mu}\end{array}$ \\
\hline Neutral Current & $\begin{array}{c}- \\
-\frac{2}{3} g_{1} s_{W} \bar{u}_{R} \gamma_{\mu} u_{R} Z_{\mu}\end{array}$ & $\begin{array}{l}g_{1} s_{W} \bar{e}_{R} \gamma_{\mu} e_{R} Z_{\mu} \\
\frac{1}{3} g_{1} s_{W} \bar{d}_{R} \gamma_{\mu} d_{R} Z_{\mu}\end{array}$ \\
\hline
\end{tabular}

Unification of the electromagnetic and weak forces into the electroweak force is thus acheived by the addition of an $\mathrm{SU}(2)_{L}$ gauge symmetry. Although this electroweak Lagrangian is able to describe most of physics around the mass scale of about a hundred Giga-ElectronVolts $(\mathrm{GeV})$, it cannot explain how the weak bosons and fermions are observed to be massive given that mass terms break $\mathrm{SU}(2)_{L}$ symmetry.

\subsection{Higgs Mechanism}

The Higgs mechanism [8, 9, 10] offers an elegant solution to spontaneously generate the mass terms for weak bosons and fermions, which otherwise violate the $\mathrm{SU}(2)_{L}$ 
symmetry. This requires adding to the electroweak Lagrangian an extra spin-0 scalar field $\Phi$ as a complex doublet:

$$
\begin{gathered}
\Phi=\left(\begin{array}{c}
\phi^{+} \\
\phi^{0}
\end{array}\right)=\frac{1}{\sqrt{2}}\left(\begin{array}{c}
\phi_{1}-i \phi_{2} \\
\phi_{3}-i \phi_{4}
\end{array}\right) \\
\mathcal{L}_{\text {Higgs }}=\left(D_{\mu} \Phi\right)^{\dagger}\left(D^{\mu} \Phi\right)+\mu^{2} \Phi^{\dagger} \Phi-\lambda\left(\Phi^{\dagger} \Phi\right)^{2}
\end{gathered}
$$

where the covariant derivative in the kinetic term is the same as the left-handed equation 2.26 with $Y_{\Phi}=+1$ as the eigenvalue of the weak hypercharge.

The particularly careful construction of the term $V(\Phi)=-\mu^{2} \Phi^{\dagger} \Phi+\lambda\left(\Phi^{\dagger} \Phi\right)^{2}$ as the potential term of the Lagrangian has some special features. If $\mu^{2}<0, V(\Phi)$ has a minimum at $\Phi=0$. If $\mu^{2}>0, V(\Phi)$ has a minimum at $\sqrt{|\Phi|^{2}}=\sqrt{\frac{\mu^{2}}{\lambda}}$ such that $V(\Phi)$ has a non-zero expectation value, and is commonly refered to as a "mexican hat" potential. It is critical to note that in this form the Lagrangian in Eq. 2.38 is manifestedly $\mathrm{SU}(2)_{L}$ invariant.

To simplify the following algebra, it's useful to work in the unitary gauge [11]. Since the Largrangian is $\mathrm{SU}(2)_{L}$ invariant, one can always make unitary transformation to this gauge that removes the upper component of $\Phi$ :

$$
\begin{aligned}
U(x) \Phi & =\frac{1}{\sqrt{2}}\left(\begin{array}{c}
0 \\
\phi(x)
\end{array}\right) \\
& =\frac{1}{\sqrt{2}}\left(\begin{array}{c} 
\\
0 \\
v+h(x)
\end{array}\right)
\end{aligned}
$$

Here, the remaining lower component has been expanded around a vacuum expectation value $v$ by a small perturbation $h(x)$. By minimizing the potential term with respect to $h(x)$, one can show that the minimum occurs at $v$, corresponding to 
the minimum $\sqrt{|\Phi|^{2}}=\frac{\mu^{2}}{\lambda}$ of $V(\Phi)$ mentioned above. By writing out the potential term, the self-interaction terms of the field $h(x)$ are revealed:

$$
\begin{aligned}
\mu^{2} \Phi^{\dagger} \Phi-\lambda\left(\Phi^{\dagger} \Phi\right)^{2} & =\frac{1}{2} \mu^{2}\left(v^{2}+h^{2}+2 v h\right)-\frac{1}{4} \lambda\left(v^{4}+h^{4}+4 v h^{3}+6 v^{2} h^{2}+4 v^{3} h\right) \\
& =v\left(\mu^{2}-\lambda v^{2}\right) h+\left(\frac{1}{2} \mu^{2}-\frac{3}{2} \lambda v^{2}\right) h^{2}-v \lambda h^{3}-\frac{1}{4} \lambda h^{4}+\mathrm{const} \\
& =\lambda v^{2} h^{2}-v \lambda h^{3}-\frac{1}{4} \lambda h^{4}+\mathrm{const}
\end{aligned}
$$

The term linear in $h$ has been removed by a substitution $\mu^{2}=\lambda v^{2}$ in the above derivation. The cubic and quartic self-interaction terms are thus revealed. Also evident is the mass term $\lambda v^{2} h^{2}$, yielding $M_{H}=\sqrt{2 \lambda} v$.

The kinetic term is written in terms of the gauge bosons in the following way:

$$
\begin{aligned}
& \left(D_{\mu} \Phi\right)^{\dagger}\left(D^{\mu} \Phi\right) \\
& =\frac{1}{2}\left|\left(\begin{array}{cc}
\partial_{\mu}-\frac{i}{2} e A_{\mu}+\frac{i}{2}\left(g_{1} s_{W}-g_{2} c_{W}\right) Z_{\mu} & -i \frac{1}{\sqrt{2}} g_{2} W_{\mu}^{+} \\
-i \frac{1}{\sqrt{2}} g_{2} W_{\mu}^{-} & \partial_{\mu}+\frac{i}{2} \sqrt{g_{1}^{2}+g_{2}^{2}} Z_{\mu}
\end{array}\right)\left(\begin{array}{c}
0 \\
v+h
\end{array}\right)\right|^{2} \\
& =\frac{1}{2}\left(\partial_{\mu} h\right)^{2}+\frac{g_{1}^{2}+g_{2}^{2}}{8} v^{2} Z_{\mu}^{2}+\frac{g_{1}^{2}+g_{2}^{2}}{8} h^{2} Z_{\mu}^{2}+\frac{g_{1}^{2}+g_{2}^{2}}{4} v h Z_{\mu}^{2}+\frac{g_{2}^{2}}{4} v^{2} W_{\mu}^{+} W^{-, \mu} \\
& +\frac{g_{2}^{2}}{4} h^{2} W_{\mu}^{+} W^{-, \mu}+\frac{g_{2}^{2}}{2} v h W_{\mu}^{+} W^{-, \mu}
\end{aligned}
$$

The mass terms $\frac{g_{2}^{2}}{4} v^{2} W_{\mu}^{+} W^{-, \mu}=M_{W}^{2} W_{\mu}^{2}, \frac{g_{1}^{2}+g_{2}^{2}}{8} v^{2} Z_{\mu}^{2}=\frac{1}{2} M_{Z}^{2} Z_{\mu}^{2}$, and $0=\frac{1}{2} M_{A}^{2} A_{\mu}^{2}$ show the $W^{ \pm}$and $Z$ bosons masses $\frac{1}{2} g_{2} v$ and $\frac{\sqrt{g_{1}^{2}+g_{2}^{2}}}{2} v$, respectively, while leaving the photon massless as observed in nature. Cubic and quartic vertices describing self-couplings between the bosons arise as well. It also predicts the ratio of $W$ and $Z$ masses to be $\frac{M_{W}}{M_{Z}}=c_{W}$, which has been experimentally verified [12]. The vacuum expectation value $v \approx 246 \mathrm{GeV}$ is obtained from these relations.

When expanded in terms of the field $h(x)$, the Lagrangian is not invariant under $\mathrm{SU}(2)_{L}$, although we started with a manifestedly invariant form of the Lagrangian. This is why the symmetry is said to have been "spontaneously" broken by expanding around $v$. 
Fermion masses are accomodated by adding interaction terms between the fermions and scalar field $\Phi$. For the down-type fermions $\psi_{L}$ and $\psi_{R}$ with corresponding Yukawa couplings $\lambda_{d}$ :

$$
\begin{aligned}
\mathcal{L}_{\text {fermion-Higgs }}^{\text {down }} & =-\lambda_{d} \bar{\Psi}_{L} \Phi \psi_{R}+\text { h.c. } \\
& =-\frac{1}{\sqrt{2}} \lambda_{d}\left(\bar{\psi}_{L}^{\prime}, \bar{\psi}_{L}\right)\left(\begin{array}{c}
0 \\
v+h
\end{array}\right) \psi_{R}+h . c . \\
& =-\frac{1}{\sqrt{2}} \lambda_{d} v \bar{\psi}_{L} \psi_{R}-\frac{1}{\sqrt{2}} \lambda_{d} \bar{\psi}_{L} \psi_{R} h+h . c . \\
& =-\frac{1}{\sqrt{2}} \lambda_{d} v \bar{\psi} \psi-\frac{1}{\sqrt{2}} \lambda_{d} \bar{\psi} \psi h
\end{aligned}
$$

Masses of down-type fermions $m_{d}=\frac{1}{\sqrt{2}} \lambda_{d} v$ and the interactions of the Higgs with fermions are obtained in this manner.

Mass terms for the up-type fermions $\psi_{L}^{\prime}$ and $\psi_{R}^{\prime}$ can similarly be generated. The final mass term is revealed rotating the Higgs field in the Lagrangian terms as follows:

$$
\begin{aligned}
\mathcal{L}_{\text {fermion-Higgs }}^{\text {up }} & =-\lambda_{u} \bar{\Psi}_{L} \Phi^{\prime} \psi_{R}^{\prime}+h . c ., \\
\Phi^{\prime} & =-i \sigma_{2} \Phi=\left(\begin{array}{c}
v+h \\
0
\end{array}\right) \\
\Rightarrow \mathcal{L}_{\text {fermion-Higgs }}^{\text {up }} & =-\frac{1}{\sqrt{2}} \lambda_{u}\left(\bar{\psi}_{L}^{\prime}, \bar{\psi}_{L}\right)\left(\begin{array}{c}
v+h \\
0
\end{array}\right) \psi_{R}^{\prime}+h . c . \\
& =-\frac{1}{\sqrt{2}} \lambda_{u} v \bar{\psi}_{L}^{\prime} \psi_{R}^{\prime}-\frac{1}{\sqrt{2}} \lambda_{u} \bar{\psi}_{L}^{\prime} \psi_{R}^{\prime} h+h . c . \\
& =-\frac{1}{\sqrt{2}} \lambda_{u} v \bar{\psi}^{\prime} \psi^{\prime}-\frac{1}{\sqrt{2}} \lambda_{u} \bar{\psi}^{\prime} \psi^{\prime} h
\end{aligned}
$$

Thus, the masses $m_{u}=\frac{1}{\sqrt{2}} \lambda_{u} v$ for both down-type and the up-type are generated from fermion-Higgs interaction. 


\subsection{QCD}

The theory of the strong interaction between quarks mediated by the gluons, via color charges, is called Quantum Chromodynamics (QCD) [13, 14]. It is described by the $\mathrm{SU}(3)_{C}$ group, which has eight spin-1 massless gluon fields $G_{\mu}^{a}(\mathrm{a}=1, \ldots, 8)$. The quarks $Q$ transform as triplets under the $\mathrm{SU}(3)_{C}$ group:

$$
\mathcal{L}_{Q C D}=\bar{Q}\left(i \gamma^{\mu} D_{\mu}-m\right) Q-\frac{1}{4} G_{\mu \nu}^{a} G_{a}^{\mu \nu}
$$

where $G_{\mu \nu}^{a}=\partial_{\mu} G_{\nu}^{a}-\partial_{\nu} G_{\mu}^{a}+g_{s} f^{a b c} G_{\nu}^{b} G_{\nu}^{c}$ is the field strength tensor of $G_{\mu}^{a}, g_{s}$ is the strong coupling, and $f^{a b c}$ are the associated structure constants of the $\mathrm{SU}(3)_{C}$ algebra:

$$
\left[t^{a}, t^{b}\right]=i f^{a b c} t^{c}
$$

The eight $t^{a}$ are one-half times the $3 \times 3$ Gell-Mann matrices $\lambda^{a}$, the generators of $\mathrm{SU}(3)_{C}$. Similar to $\mathrm{SU}(2)_{L}$, the non-commuting $\mathrm{SU}(3)_{C}$ algebra results in selfinteraction among the gluons fields.

In terms of number of colors $N_{C}=3$ and the strong coupling constant $\alpha_{s}=\frac{g_{s}^{2}}{4 \pi}$, the structure of QCD gives rise to three types of vertices whose square gives the following three basic amplitudes:

- gluon bremsstrahlung from quarks with strength $=\frac{N_{C}^{2}-1}{2 N_{C}} \cdot \alpha_{S}=\frac{4}{3} \alpha_{S}$,

- gluon bremsstrahlung from gluons with strength $=N_{C} \cdot \alpha_{S}=3 \alpha_{S}$, and

- gluon splitting into a quark-antiquark pair with strength $=\frac{1}{2} \alpha_{S}$.

\subsection{Higgs boson at the LHC}

There are four main production modes of the Higgs boson at the LHC: the Feynman diagrams for which are shown in Figure 2.1. 
- The dominant mode is gluon-gluon fusion ( $g g \rightarrow H$, denoted $g g F)$, in which a top- and b-quark loop mediates an effective coupling between the Higgs and two initial state gluons.

- The Vector Boson Fusion (VBF) production mode occurs through the process $q \bar{q} \rightarrow q^{\prime} \bar{q}^{\prime} V^{(*)} V^{(*)} \rightarrow q^{\prime} \bar{q}^{\prime} H\left(V^{(*)}=W^{(*)}, Z^{(*)}\right)$, where the vector bosons are radiated off of two initial state quarks and fuse to give a Higgs boson.

- There is a mode where a Higgs is produced in association with a vector boson $(V H, V=W, Z)$, also known as Higgs-strahlung, which occurs through the process $q \bar{q} \rightarrow V^{*} \rightarrow V H$.

- Finally, there is the $t \bar{t} H$ mode which proceedes through the process $g g / q \bar{q} \rightarrow$ $t \bar{t} H$.

The production cross sections versus the mass of the Higgs Boson $\left(m_{H}\right)$ for these processes at the center-of-mass energies of 8 and $13 \mathrm{TeV}$ are shown in Figure 2.2. The cross section for the major production processes for $m_{H}=125 \mathrm{GeV}$ is shown in Table $2.2[15]$.

\begin{tabular}{|c|c|c|c|c|c|}
\hline \multicolumn{6}{|c|}{ Production cross sections $\left(\mathrm{pb}, m_{H}=125 \mathrm{GeV}\right)$} \\
\hline & $\operatorname{ggF}$ & $\overline{\mathrm{VBF}}$ & WH & $\mathrm{ZH}$ & $t \bar{t} H$ \\
\hline 7 & 15.32 & 1.222 & 0.5729 & 0.3158 & 0.0863 \\
\hline 8 & 19 & 1.578 & 0.6966 & 0.3943 & 0.1302 \\
\hline $13 \mathrm{Te}$ & 43.92 & 3.748 & 1.380 & 0.8696 & 0.5085 \\
\hline $14 \mathrm{TeV}$ & 49.85 & 4.180 & 1.504 & 0.8830 & 0.6113 \\
\hline
\end{tabular}

Table 2.2: Cross sections for the major production processes assuming $m_{H}=125$ $\mathrm{GeV}$

For a Higgs boson with a mass of $125 \mathrm{GeV}$, the SM predicts a mean life time of about $1.6 \times 10^{-22} \mathrm{~s}$. The life time of Higgs boson being very small at masses larger than a few $\mathrm{GeV}$, it decays immediately into final state fermion or boson pairs. The decay into pairs of bosons can be divided into two-, three-, and four-body final 


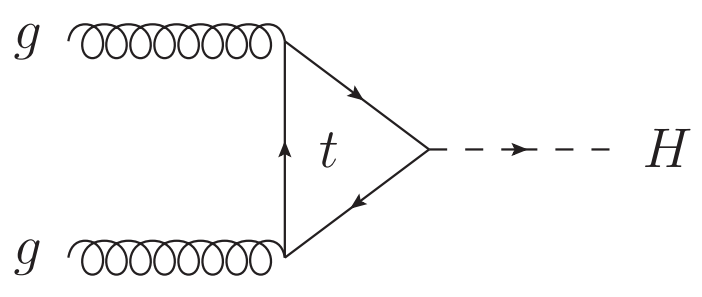

(a) $g g F$

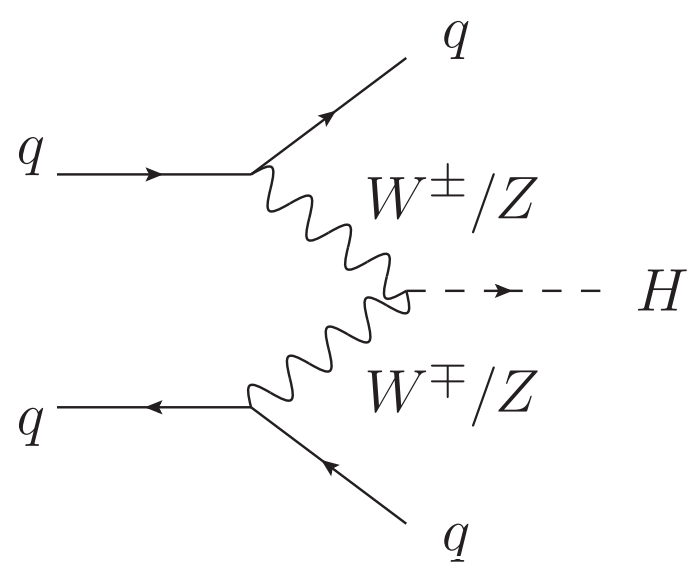

(c) $\mathrm{VBF}$

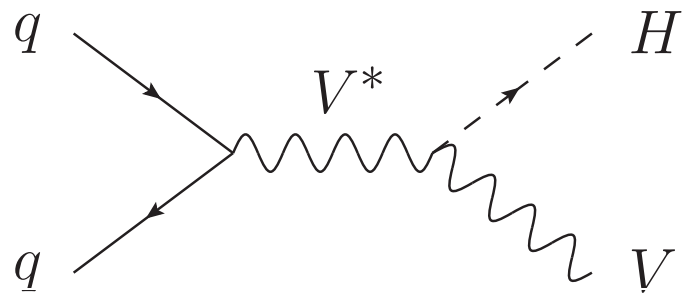

(b) $\mathrm{VH}$

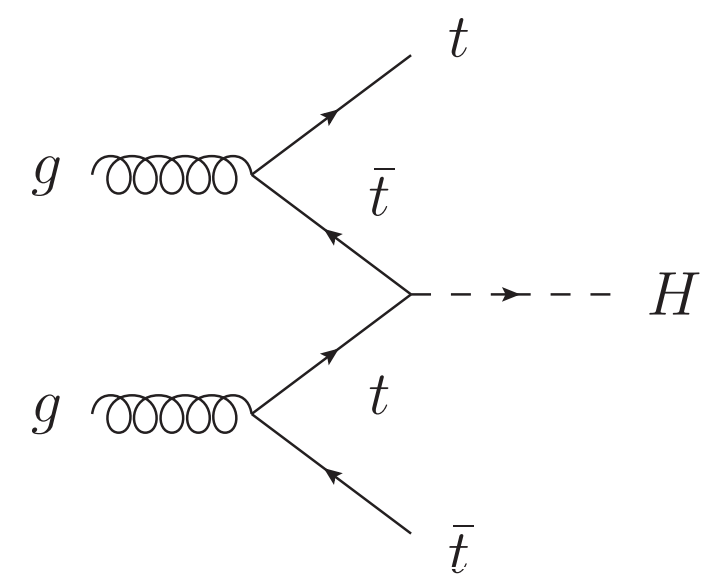

(d) $\mathrm{ttH}$

Figure 2.1: Feynman diagrams for the Higgs production modes accessible at the LHC 

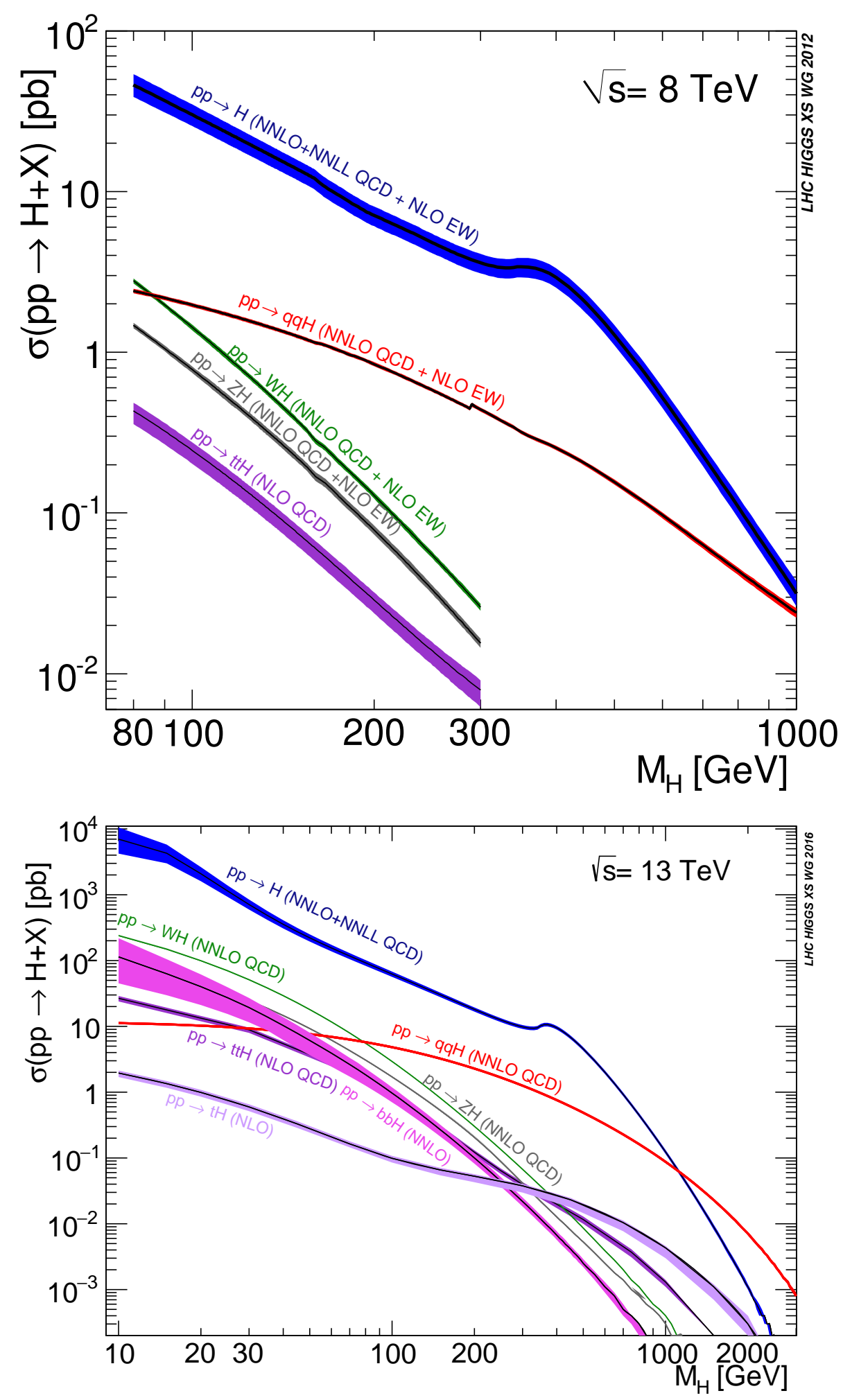

Figure 2.2: Production cross section for various production modes as a function of the Higgs mass $m_{H}$ for both 8 and $13 \mathrm{TeV}$ center-of-mass energies [16]. 


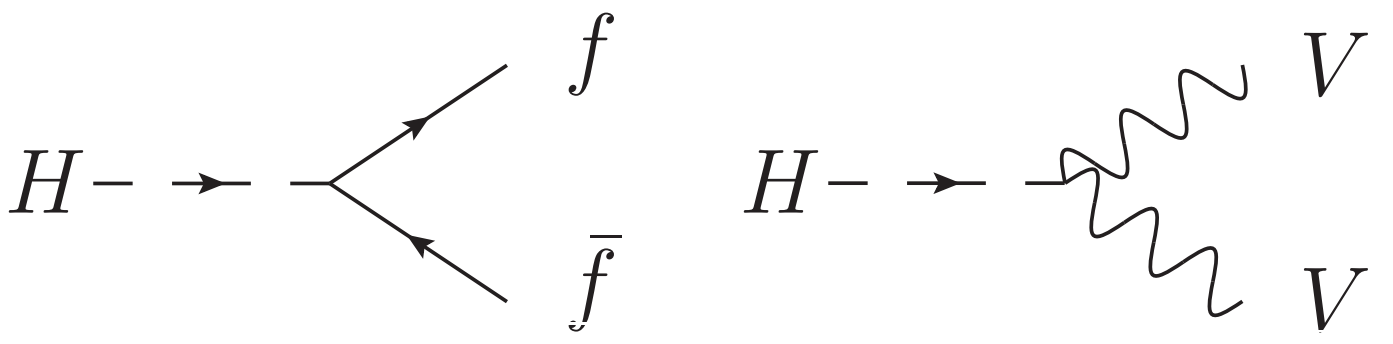

(a) $H \rightarrow f \bar{f}$

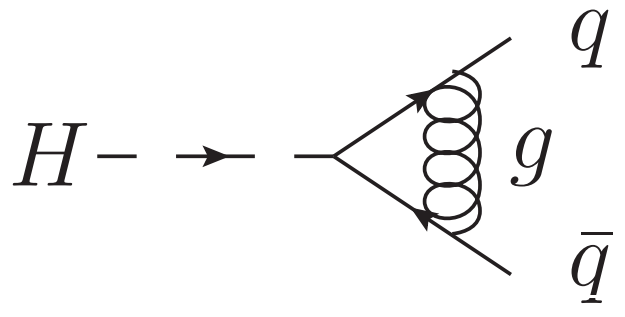

(c) $H \rightarrow q \bar{q}$ with QCD correction

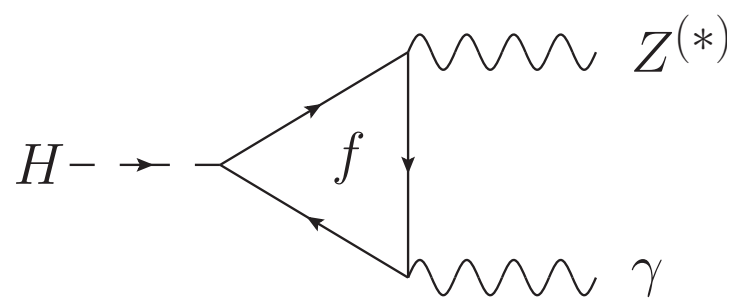

(e) $H \rightarrow Z^{(*)} \gamma$ (b) $H \rightarrow V V$

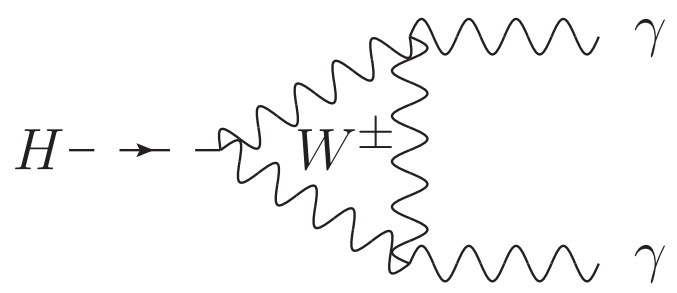

(d) $H \rightarrow \gamma \gamma$

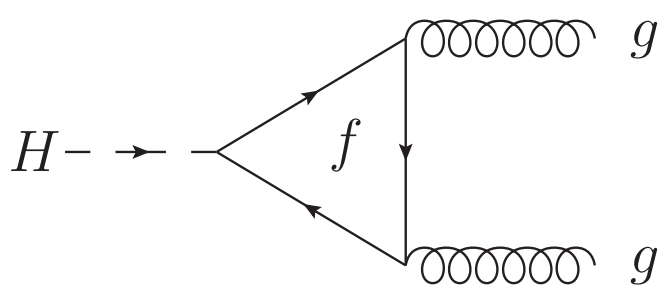

(f) $H \rightarrow g g$

Figure 2.3: Possible Higgs boson decays in the SM: (a) shows the leading-order decay into fermion pairs, (b) shows the two-body decay into vector bosons, (c) shows decay the into light quarks with QCD corrections, (d) shows the three-body decay into photon pairs through a $W^{ \pm, *}$ loop, (e) shows the decay into $Z^{(*)} \gamma$ through a fermion loop, and (f) shows the decay into gluon pairs through a top-quark loop.

state decays, while other decays into photon pairs, gluon pairs, or $Z+$ photon proceeds through loop vertices. Feynman diagrams for possible decays are shown in Figure 2.3.

The leading-order partial width to fermions can be written as a function of the fermion mass $[17,18]$ :

$$
\Gamma(H \rightarrow f \bar{f})=\frac{1}{4 \pi \sqrt{2}} G_{\mu} N_{c} m_{H} m_{f}^{2} \beta_{f}^{3}
$$

where $G_{\mu}$ is the Fermi constant, $N_{c}$ is the color factor, which is 3 (1) for quarks 

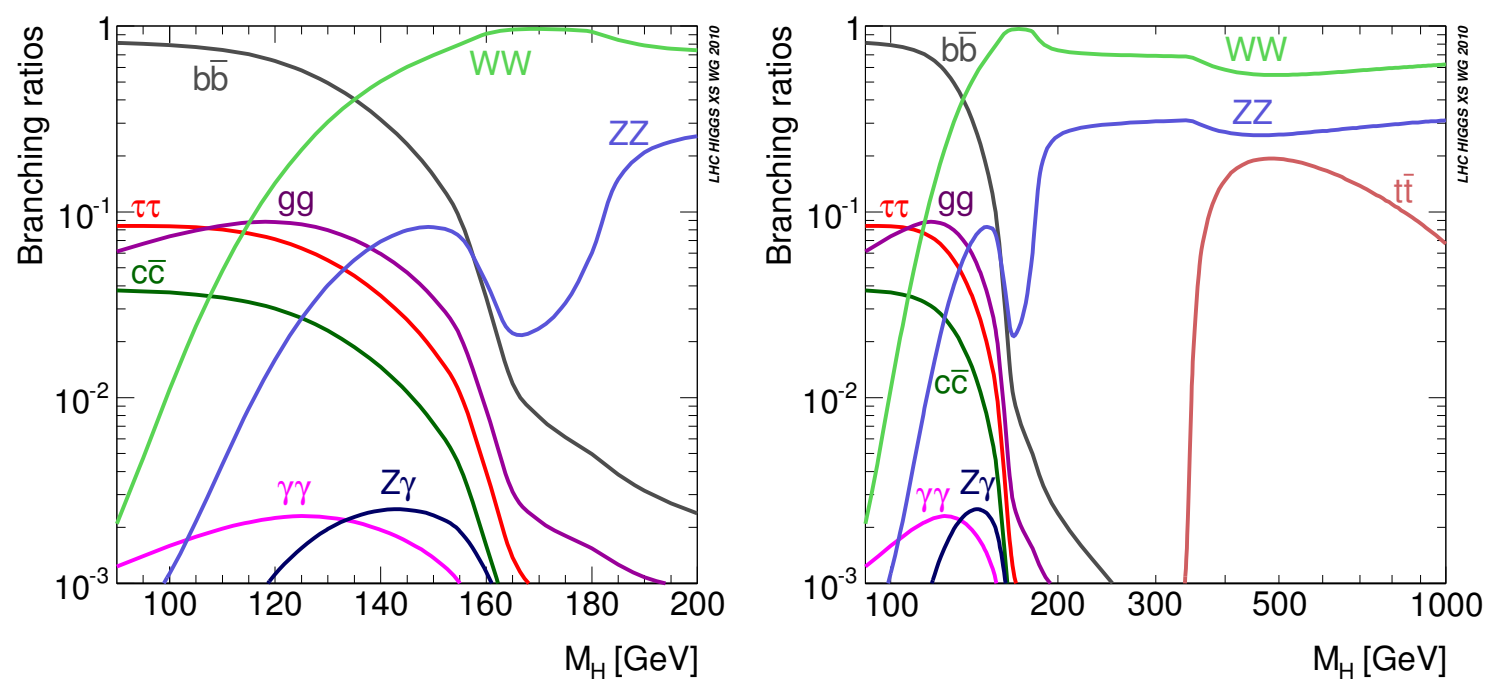

Figure 2.4: Branching ratio for various decay modes as a function of the Higgs mass $m_{H}$. The range of the left plot has an upper bound of $200 \mathrm{GeV}$ to reveal the structure of the curves at low $m_{H}[19]$.

(leptons), $m_{f}$ is the mass of the fermion, and $\beta_{f}=\left(1-\frac{4 m_{f}}{m_{H}}\right)^{1 / 2}$ is the fermion velocity in the final state.

The branching ratio to any single mode is the ratio of the partial width to the total width, where the total width is the sum of all possible partial widths:

$$
\mathcal{B}(H \rightarrow X X)=\frac{\Gamma(H \rightarrow X X)}{\sum_{i} \Gamma\left(H \rightarrow X_{i} X_{i}\right)}
$$

The branching fraction for various modes as a function of the Higgs mass is shown in Figure 2.4. The values of the branching ratios for various decay modes assuming $m_{H}=125$ are shown in Table 2.3.

\begin{tabular}{ccccccc}
\hline \hline \multicolumn{7}{c}{ Branching ratios $\left(m_{H}=125 \mathrm{GeV}\right)$} \\
\hline$H \rightarrow W W^{(*)}$ & $H \rightarrow \gamma \gamma$ & $H \rightarrow Z Z^{(*)}$ & $H \rightarrow b \bar{b}$ & $H \rightarrow \tau^{+} \tau^{-}$ & $H \rightarrow Z \gamma$ & $H \rightarrow g g$ \\
\hline 0.215 & 0.00228 & 0.0264 & 0.577 & 0.0632 & 0.00154 & 0.0857 \\
\hline
\end{tabular}

Table 2.3: Branching ratios for various decay modes assuming $m_{H}=125 \mathrm{GeV}$ [19] 


\subsection{Lepton flavour violating decays}

Following the discovery of the Higgs Boson at LHC [20, 21], there has been considerable interest in search for its exotic decay modes. The lepton flavour violating (LFV) decays are unambiguous signatures which predict the discovery of new physics beyond Standard Model (BSM). LFV decays of Higgs boson are absent in the SM, but highly anticipated in a large class of new physics models.

The Yukawa Largangian describing the $Y_{i j}^{a}$ couplings between multiple Higgs fields $\left(h^{a}\right)$ to a pair of fermions $\left(f^{i}, f^{j}\right)$ is given by $[22,23]$ :

$$
\mathcal{L}_{Y}=-m_{i} \bar{f}_{L}^{i} f_{R}^{i}-Y_{i j}^{a}\left(\bar{f}_{L}^{i} f_{R}^{j}\right) h^{a}+\text { h.c. }
$$

where the index $a$ runs over all the scalars and $Y_{i j}^{a}$ is imaginary for pseudoscalar Higgs.

In a single Higgs theory $\left(h^{a}=H\right)$, partial widths, $\Gamma$, are related to the couplings by ${ }^{1}$ :

$$
\Gamma(H \rightarrow \ell \tau)=\frac{m_{H}}{8 \pi}\left(\left|Y_{\tau \ell}\right|^{2}+\left|Y_{\ell \tau}\right|^{2}\right)
$$

Using $m_{H}=125 \mathrm{GeV}$ and $\Gamma_{\mathrm{SM}}=4.1 \mathrm{MeV}[24]$, the branching ratio $(\mathcal{B})$ is defined as:

$$
\mathcal{B}(H \rightarrow \ell \tau)=\frac{\Gamma(H \rightarrow \ell \tau)}{\Gamma(H \rightarrow \ell \tau)+\Gamma_{\mathrm{SM}}}
$$

Non-diagonal Yukawa couplings can also induce LFV decays: $\tau \rightarrow \ell \gamma$, partial width of which can be calculated as [23]:

$$
\left.\Gamma(\tau \rightarrow \ell \gamma)=\frac{\alpha m_{\tau}^{5}}{64 \pi^{4}}\left(\left|c_{L}\right|^{2}+\left|c_{R}\right|^{2}\right)\right)
$$

\footnotetext{
${ }^{1}$ Unless explicitly mentioned otherwise, leptons (denoted by $\ell$ or $\ell^{\prime}$ ) refer to electrons or muons althroughout this thesis.
} 
where the Wilson coefficients have been calculated to one loop as:

$$
c_{L}^{1 \text { loop }} \simeq \frac{1}{12 m_{h}^{2}} Y_{\tau \tau} Y_{\tau \mu}^{*}\left(-4+3 \log \frac{m_{h}^{2}}{m_{\tau}^{2}}\right), \quad c_{R}^{1 \text { loop }} \simeq \frac{1}{12 m_{h}^{2}} Y_{\mu \tau} Y_{\tau \tau}\left(-4+3 \log \frac{m_{h}^{2}}{m_{\tau}^{2}}\right)
$$

The 2-loop contributions are obtained numerically to be as:

$$
c_{L}^{2 \text { loop }}=Y_{\tau \mu}^{*}\left(-0.082 Y_{t t}+0.11\right) \frac{1}{(125 \mathrm{GeV})^{2}}=0.055 Y_{\tau \mu}^{*} \frac{1}{(125 \mathrm{GeV})^{2}},
$$

where in the last step the top Yukawa coupling is taken as $Y_{t t}=\left(Y_{t t}\right)_{S M}=\bar{m}_{t} / v=$ 0.67, and the results have been normalized to $m_{h}$ for easier comparison. Here $\bar{m}_{t}$ denotes the top quark mass parameter in the $\overline{\mathrm{MS}}$ renormalization scheme, $\bar{m}_{t} \simeq$ $164 \mathrm{GeV}$.

The current experimental bounds on LFV decays of the $\tau$ lepton $\mathcal{B}(\tau \rightarrow \mu \gamma)<$ $4.4 \times 10^{-8}[25]$ can thus be used to predict an indirect limit on the LFV decays of the Higgs boson $\mathcal{B}(H \rightarrow \mu \tau) \simeq 10 \%$, which is within the reach of experimental sensitivity of the ATLAS experiment at the Large Hadron Collider (LHC). In this thesis, direct searches for LFV decays $H \rightarrow e \tau$ and $H \rightarrow \mu \tau$ of the Higgs boson are presented. 


\section{CHAPTER III}

\section{LHC \& ATLAS}

\subsection{The Large Hadron Collider}

The Large Hadron Collider (LHC) is located at the European Organization for Nuclear Research (CERN), just outside of Geneva, on the border of France and Switzerland. The LHC is the world's largest particle collider, designed to produce high energy proton-proton and heavy ion collisions [26].

There are four primary detectors along the ring, shown in Figure 3.1: ALICE, LHCb, ATLAS, and CMS. ALICE (A Large Ion Collider Experiment) is designed to look at heavy ion collisions. LHCb (Large Hadron Collider beauty) is a dedicated b-physics detector that operates at low luminosity. ATLAS (A Toroidal LHC ApparatuS) and CMS (Compact Muon Solenoid) are general purpose detectors with design goals to find new physics in proton-proton interactions, but are also capable of reconstructing heavy ion collisions and b-quark decays.

The LHC began operation in 2010. Data with substantial luminosity profile has been taken at the center of mass energies $\sqrt{s}=7 \mathrm{TeV}$ and $8 \mathrm{TeV}$ during 2011 and 2012, respectively, referred to as Run 1. The LHC had a long shutdown from 2013 until 2014, at which time it underwent upgrades aimed to increase the center of mass energy. From 2015 to 2018, it operated at a center of mass energy of $13 \mathrm{TeV}$, referred to as Run 2. At the end of 2018, it entered a two-year shutdown period to further 


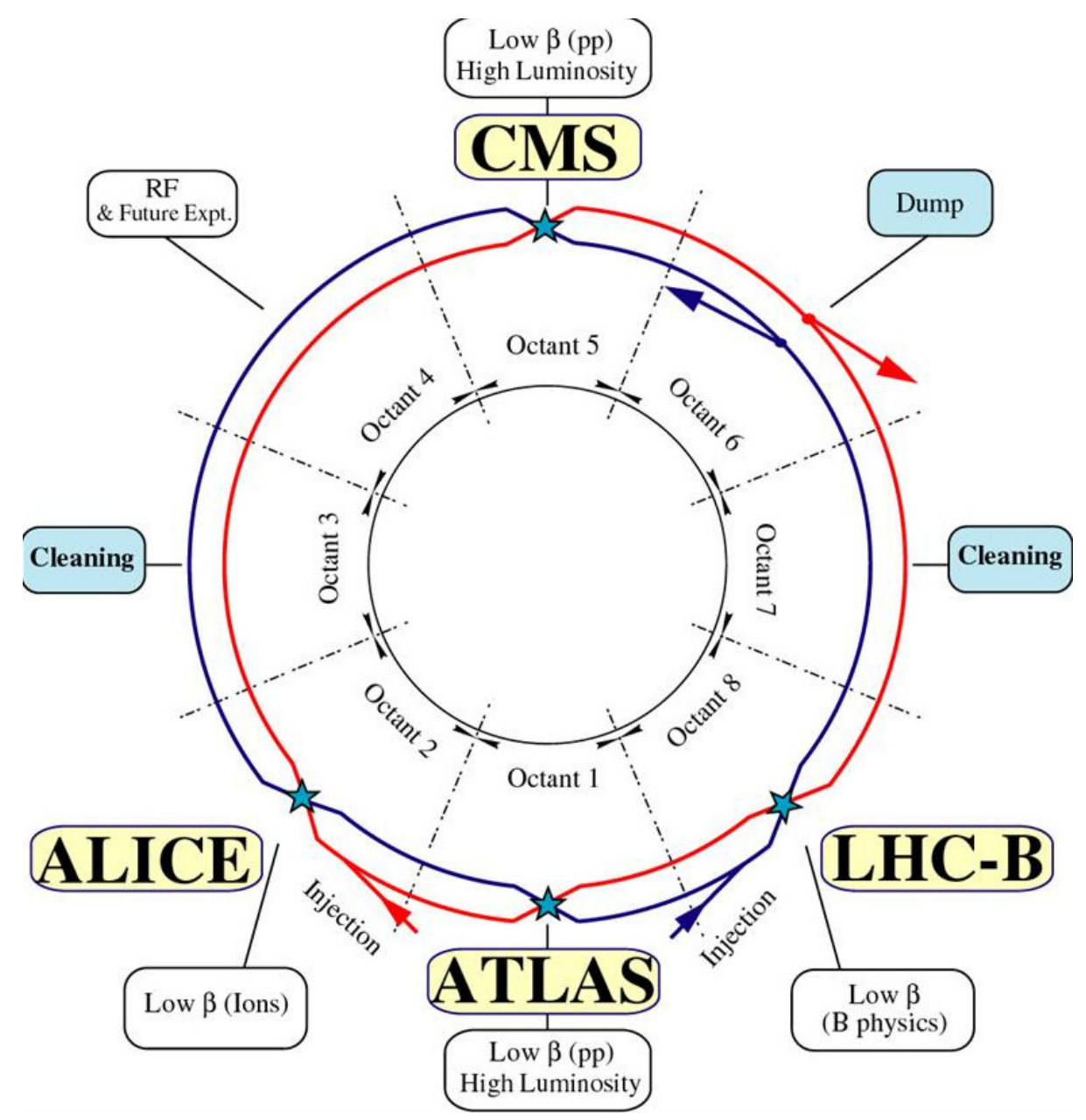

Figure 3.1: Layout of the LHC ring showing the positions of each experiment at the collision points as well as injection, beam dump, and beam cleaning regions(Courtesy: Reference [27]).

upgrade the center of mass energy to $14 \mathrm{TeV}$.

The circulating proton beams in the LHC cross paths at the four experimental interaction points where the main LHC experiments are located. The design instantaneous luminosity $\mathcal{L}$ at LHC is $10^{34} \mathrm{~cm}^{-2} \mathrm{~s}^{-1}=10 \mathrm{nb}^{-1} \mathrm{~s}^{-1}$, given as a function of the beam parameters:

$$
\mathcal{L}=\frac{N_{b}^{2} n_{b} f_{\text {rev }} \gamma_{r}}{4 \pi \epsilon_{n} \beta^{*}} F
$$

where $N_{b}$ is the number of particles collected together as a bunch, $n_{b}$ is the number of bunches per beam, $f_{\text {rev }}$ is the revolution frequency, $\gamma_{r}$ is the relativistic Lorentz factor of the beam, $\epsilon_{n}$ is the normalized transverse beam emittance (area in position- 
momentum phase space), $\beta^{*}$ is the beta function at the collision point. The beta function depends on the bunch cross section, $\sigma$, and the transverse beam emittance, $\epsilon, \beta=\pi \sigma^{2} / \epsilon$. The beams are squeezed as they approach the interaction point, decreasing the amplitude such that $\beta^{*}$ is a smaller value of $\beta$ than at other points. Finally, the $F$ is the geometric luminosity reduction factor due to the crossing angle at the interaction point, given by:

$$
F=\left(1+\left(\frac{\theta_{c} \sigma_{z}}{2 \sigma^{*}}\right)\right)^{-1 / 2}
$$

where $\theta_{c}$ is the full crossing angle at the interaction point, $\sigma_{z}$ is the RMS bunch length, and $\sigma^{*}$ is the transverse RMS beam size at the interaction point [26].

Nominal design values for these quantities are given in the Table 3.1, which shows the successful operations of the accelerator teams' at the LHC during Run 2 [26, 28].

\begin{tabular}{llr}
\hline Parameter & Symbol & LHC Run 2 Value \\
\hline LHC circumference & & $26,659 \mathrm{~m}$ \\
LHC design beam energy & & $7 \mathrm{TeV}$ \\
LHC beam energy in Run 2 & $6.5 \mathrm{TeV}$ \\
Number of protons per bunch & $N_{b}$ & $1.15 \times 10^{11}$ \\
Number of proton bunches per beam & $n_{b}$ & 2,808 \\
Revolution frequency & $f_{\mathrm{rev}}$ & $11.245 \mathrm{kHz}$ \\
Lorentz factor (design) & $\gamma_{r}$ & 7462.69 \\
Lorentz factor at $\sqrt{s}=13 \mathrm{TeV}$ & & 6929.64 \\
Normalized transverse beam emittance & $\epsilon_{n}$ & $3.75 \mu \mathrm{m}$ \\
Collision point beta function & $\beta^{*}$ & $0.55 \mathrm{~m}$ \\
Full crossing angle & $\theta_{c}$ & $285 \mu \mathrm{rad}$ \\
RMS bunch length & $\sigma_{z}$ & $7.55 \times 10^{-2} \mathrm{~m}$ \\
Transverse RMS beam size & $\sigma^{*}$ & $16.6 \mu \mathrm{m}$ \\
\hline Peak design machine luminosity at 14 TeV & $\mathcal{L}$ & $10 \mathrm{nb}^{-1} \mathrm{~s}^{-1}$ \\
Peak design machine luminosity at 13 TeV & & $9 \mathrm{nb}^{-1} \mathrm{~s}^{-1}$ \\
Peak ATLAS recorded machine luminosity & & $21 \mathrm{nb}^{-1} \mathrm{~s}^{-1}$ \\
\hline
\end{tabular}

Table 3.1: Nominal design values of LHC operations parameters at ATLAS for $25 \mathrm{~ns}$ bunch crossing spacing. Design and ATLAS recorded values of the machine luminosity are also given for LHC Run 2 operations. 


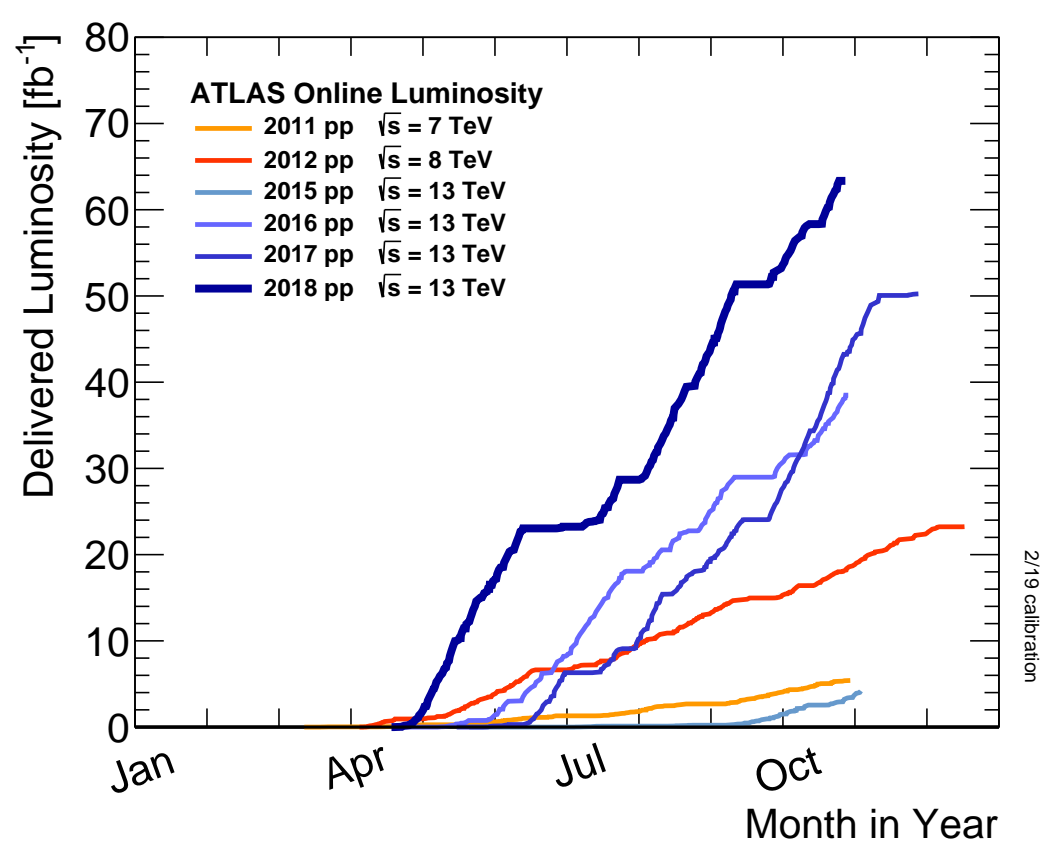

Figure 3.2: Delivered Luminosity versus time for 2011-2018 (p-p data only) (Courtesy: Reference [29]).

The cumulative luminosity versus day delivered to ATLAS during stable beams during 2011-2018 and for high energy p-p collisions is shown in Figure 3.2. The work in this thesis was done with $36.1 \mathrm{fb}^{-1}$ of $13 \mathrm{TeV}$ of data collected with the ATLAS detector during 2015 and 2016.

\section{$3.2 \quad$ ATLAS Detector}

The ATLAS detector $[30,31]$ is one of two general purpose detectors on the LHC ring. ATLAS is the largest of the LHC experiments with dimensions of $44 \mathrm{~m}$ in length and $25 \mathrm{~m}$ in height.

The layout of the detector is shown in Figure 3.3. Starting from the beam pipe and working outward, ATLAS is comprised of an inner detector inside a superconducting solenoid magnet for vertex and track reconstruction, a liquid argon (LAr) electromagnetic (EM) calorimeter for EM particle energy measurement, LAr and tile hadronic calorimeters for hadronic particle energy measurement, and a muon spectrometer for 


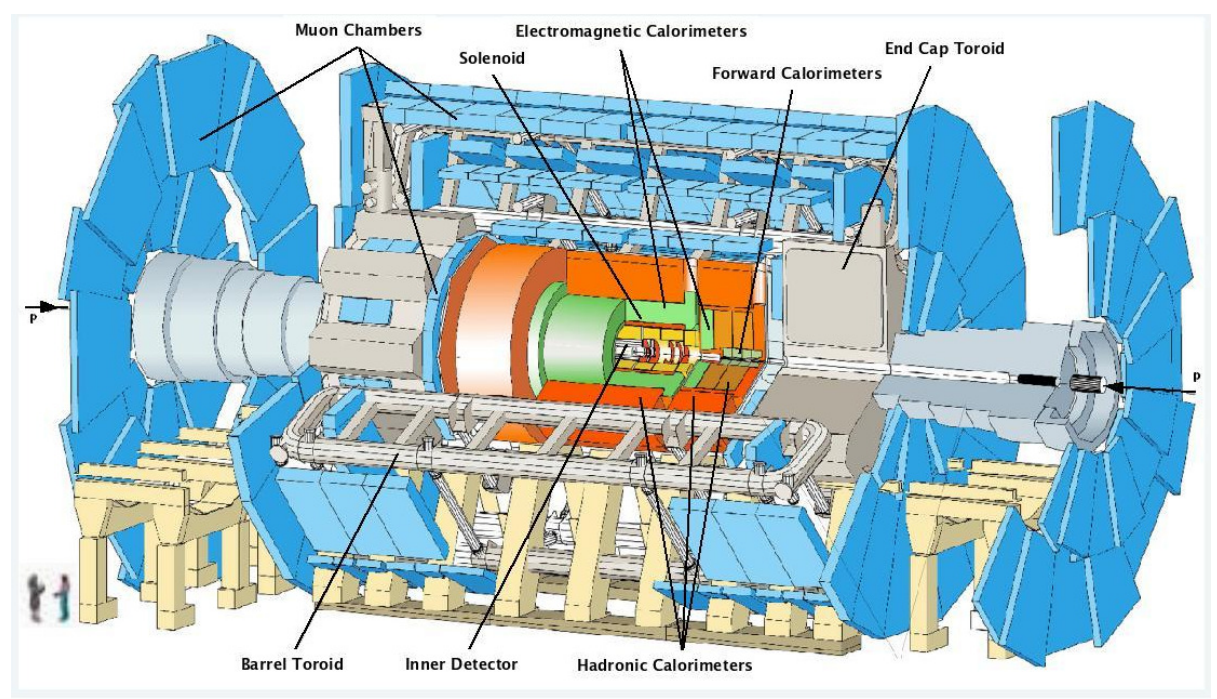

Figure 3.3: Layout of the ATLAS detector showing the major subdetectors (Courtesy: Reference [32]).

the reconstruction of muon tracks.

The inner detector is surrounded by a $2 \mathrm{~T}$ superconducting solenoid magnet and provides excellent tracking coverage in $|\eta|<2.5$. The inner detector is further bracketed at each end by end-cap toroid magnets and the entire barrel of the detector out through the calorimeters is enclosed in a toroid magnet system. These two toroid systems are both constructed such that they exhibit an eight-fold azimuthal symmetry.

The following detector coordinate directions are applied: the $z$ axis points along the beam axis; $x$ and $y$ axes define a plane perpendicular to the beam axis; $\phi$ is the azimuthal angle around the beam axis and $\theta$ is the polar angle from the beam axis. The positive $x$ direction is defined as from the interaction point to the centre of the LHC ring and the positive $y$ direction as upwards. It is more common for the angle in $\theta$ to be expressed in terms of pseudorapidity, $\eta=-\ln \left[\tan \left(\frac{\theta}{2}\right)\right]$. This is a useful spatial coordinate as it approximates to a particles rapidity, $\frac{1}{2} \ln \left(\frac{E+p_{z}}{E-p_{z}}\right)$ for light particles. The rapidity difference between two particles is Lorentz invariant under transformations along the longitudinal axis. 


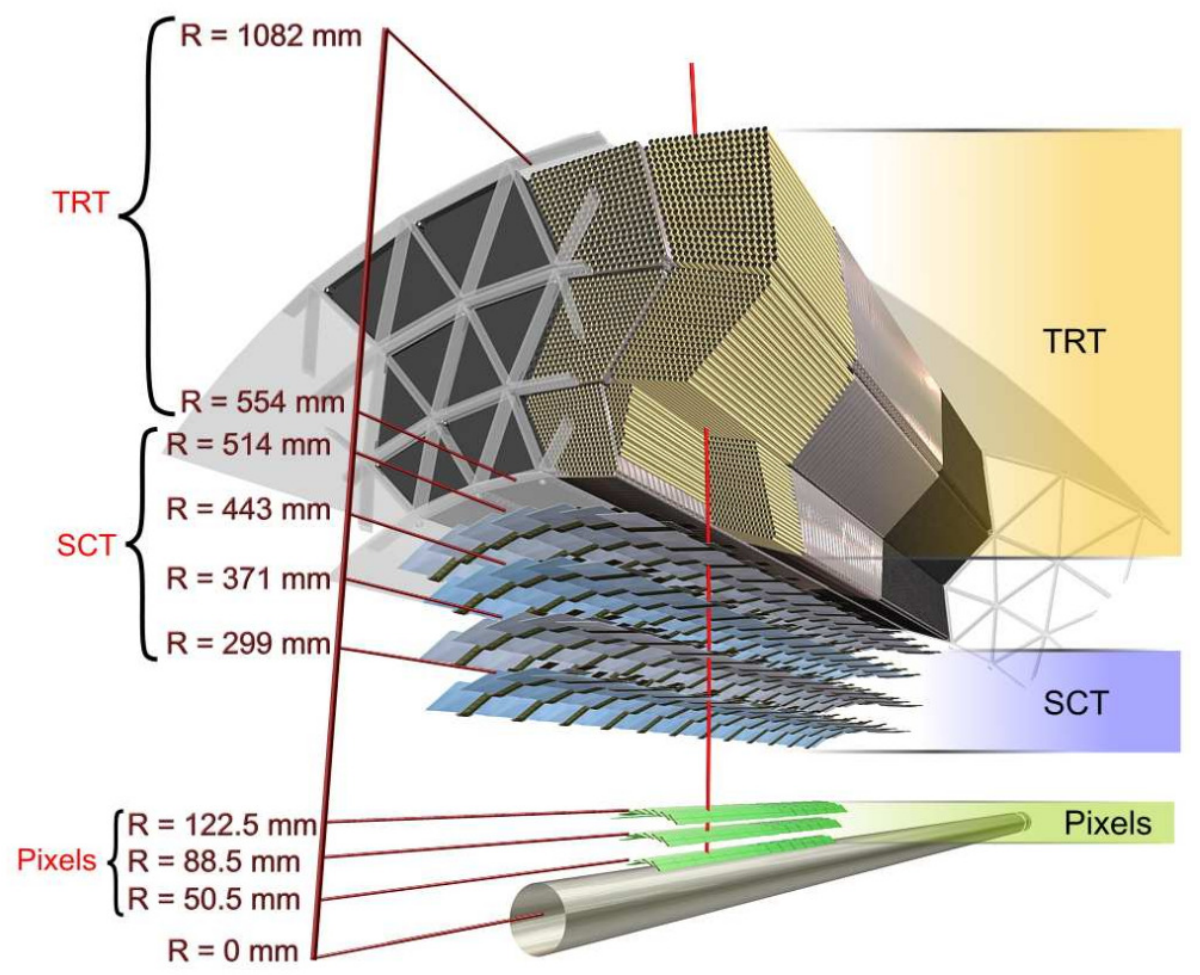

Figure 3.4: Layout of the inner detector in the barrel showing the Pixel, SCT, and TRT detectors and their geometrical configuration (Courtesy: Reference [33]).

\subsubsection{Inner Detector}

The ATLAS inner detector (ID) is designed to provide accurate reconstruction of the charged particle tracks used for the high resolution momentum measurements and particle identification, as well as the reconstruction of the primary and secondary vertices. As shown in Figure 3.4, the ID consists of three complementary sub-systems, the Pixel Detector (PD), Silicon Semiconductor Tracker (SCT) and Transition Radiation Tracker (TRT). The latter also provides an electron identification for $0.5 \mathrm{GeV}$ $<p_{T}<150 \mathrm{GeV}$ in the region of $|\eta|<2.0$. All sub-systems are fully contained within the homogeneous magnetic field of $2 \mathrm{~T}$ produced by the ATLAS solenoid magnet. Parameters of each subsystem along with typical resolution are listed in Table 3.2. 


\begin{tabular}{llcc}
\hline System & Position & Resolution $(\mu \mathrm{m})$ & $\eta$ coverage \\
\hline Pixel detector & 1 B-layer & $r-\phi=12, z=66$ & \pm 2.5 \\
& 2 barrel layers & $r-\phi=12, z=66$ & \pm 1.7 \\
& 3 end-cap disks (each side) & $r-\phi=12, z=77$ & $1.7-2.5$ \\
\hline SCT & 4 barrel layers & $r-\phi=16, z=580$ & \pm 1.4 \\
& 9 end-cap wheels (each side) & $r-\phi=16, z=580$ & $1.4-2.5$ \\
\hline TRT & Axial barrel straws & 170 (per straw) & \pm 0.7 \\
& Radial end-cap straws & 170 (per straw) & $0.7-2.5$ \\
\hline
\end{tabular}

Table 3.2: Parameters of the Inner Detector for each subsystem. For the resolution, typical values are quoted [34]. The actual resolution in each detector depends on the impact angle.

\subsubsection{Calorimeter}

The ATLAS calorimeter system, shown in Figure 3.5, provides excellent energy deposition measurements for particles with coverage up to $|\eta|<4.9$ with different calorimetry subsystems for various physics processes. In the pseudorapidity range of the inner detector $(|\eta|<2.5)$ the high granularity electromagnetic (EM) liquid argon calorimeter system provides measurement of electrons and photons. The more coarse resolution of the hadronic calorimeter systems provides measurements for jet reconstruction and missing transverse momentum, in conjunction with the large pseudorapidity coverage. These calorimeter designs are both "sampling calorimeters," where the "active" materials that provide the signals are different from the "absorber" materials that reduce the particle energy and cause showering. The calorimeter subsystems are also designed to be sufficiently thick as to contain the electromagnetic and hadronic showers that originate inside them, and to limit punch-through to the muon systems.

The electromagnetic calorimeter system consists of lead-liquid argon detectors with a characteristically unique "accordion" lead absorber plate design that allows for continuous coverage in $\phi$ with folding angles of the accordion "waves" that vary with the radius to keep the gap constant. Liquid argon (LAr) is the active detector 
material for the EM calorimeters as it has a linear behavior, very stable response over time, and is intrinsically radiation-hard. In the barrel region the LAr EM calorimeter is split into symmetric half-barrels, and in the end-caps the LAr EM calorimeter exists as two coaxial wheels, respectively covering the regions of $1.375<|\eta|<2.5$ and $2.5<|\eta|<3.2$.

The hadronic calorimeter system is composed of the tile calorimeters in the barrel region, and the LAr Hadronic End-cap Calorimeter (HEC) and LAr Forward Calorimeter (FCal) in the end-cap region. The tile sampling calorimeter resides outside the EM calorimeter system and provides coverage to $|\eta|<1.7$ and radial coverage from $2.28 \mathrm{~m}$ to $4.25 \mathrm{~m}$. The tile calorimeter absorber material is steel and uses scintillating tiles as the active material, which are read out using wavelength shifting fibers into photomultiplier tubes. The HEC exists as two wheels in each end-cap behind the end-cap EM calorimeter, extending the coverage in the end-caps to $|\eta|<3.2$. The copper absorber plates of the HEC are interleaved with $8.5 \mathrm{~mm}$ spacers of LAr providing active material. The FCal extends coverage from $3.1<|\eta|<4.9$ and is composed of three modules in each end-cap: a copper module optimized for electromagnetic measurements, and then two made of tungsten for hadronic interaction measurements. The modules are a metal matrix with regularly spaced longitudinal channels consisting of tubes with a concentric rod and LAr filling the gap between them.

Parameters of each subsystem along with typical resolution are listed in Table 3.3.

\begin{tabular}{|c|ccc|}
\hline Calorimeter & ECAL & HCAL & FCal \\
\hline Energy resolution $\frac{\sigma(E)}{E}$ & $\frac{10 \%}{\sqrt{E[\mathrm{GeV}]}} \oplus 0.7 \%$ & $\frac{5 \%}{\sqrt{E[\mathrm{GeV}]}} \oplus 3 \%$ & $\frac{100 \%}{\sqrt{E[\mathrm{GeV}]}} \oplus 10 \%$ \\
\hline
\end{tabular}

Table 3.3: The design energy resolution of the ECAL, HCAL (barrel and end-caps) and the FCal $[36,37]$. Here the symbol $\oplus$ means that the errors have been added in quadrature. 


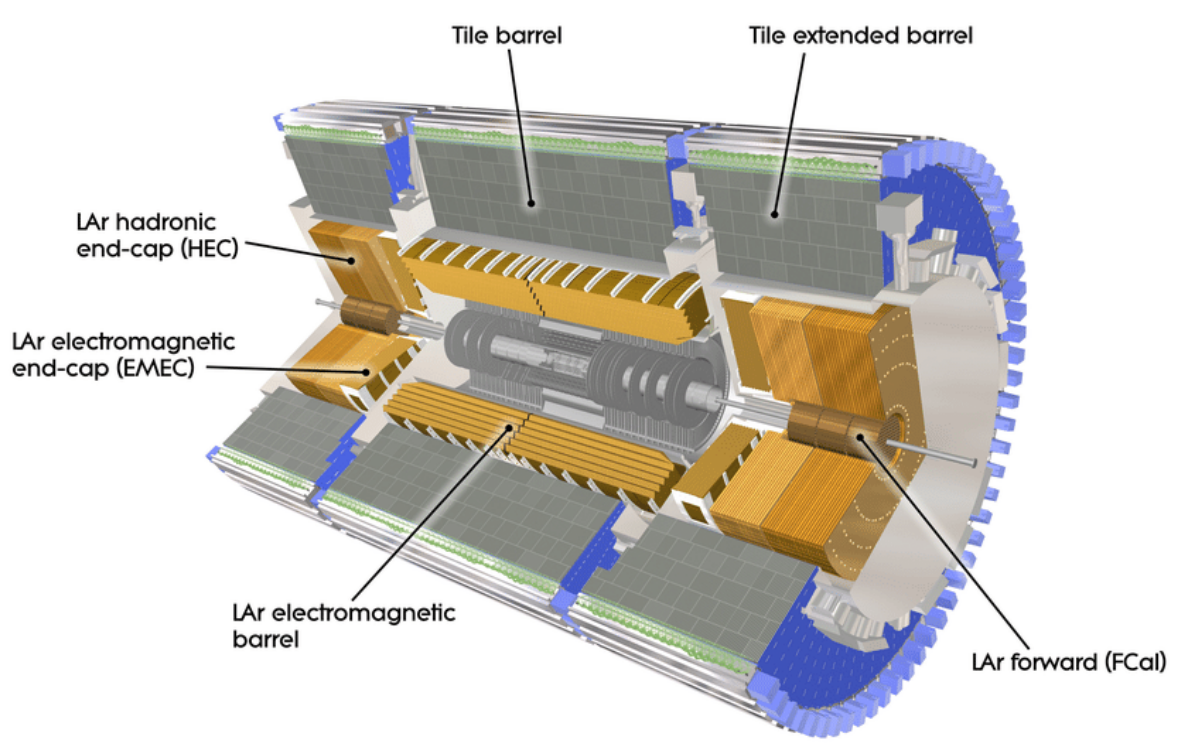

Figure 3.5: Layout of the calorimetry system (Courtesy: Reference [35]).

\subsubsection{Muon Spectrometer}

The ATLAS Muon Spectrometer measures coincident hits from particles that are curved within the field of the toroidal magnet. The spectrometer consists of a barrel that extends to $|\eta|=1.05$ and two end-caps that cover the range $1.05<|\eta|<2.7$ Muon momenta are measured with precision in three layers of monitored drift tubes (MDT). Additionally an inner layer exists equipped with cathode strip chambers (CSC), which each consist of four sensitive layers. Three doublet layers of resistive plate chambers (RPC) covering $|\eta|<1.05$ together with one triplet layer and two doublet layers of thin gap chambers (TPC) covering $1.05<|\eta|<2.4$ are used for triggering and the measurement of $\eta$ and $\phi$ coordinates. These detectors sit above or below the MDT chambers as in Figure 3.6.

\subsection{Trigger System}

The trigger system is an essential component of any hadron collider experiment. It is responsible for deciding whether or not to record a given beam crossing for later 


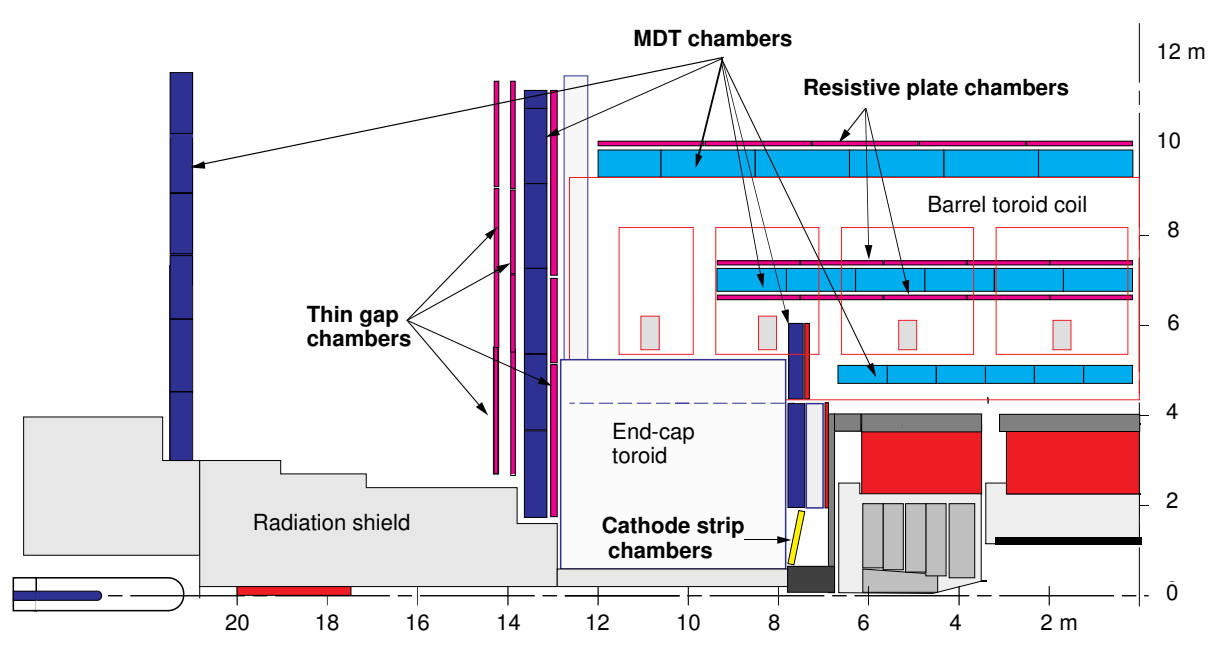

Figure 3.6: Cross section of the muon spectrometer in both barrel and end-cap [38].

study. Due to the high bunch crossing rate of $40 \mathrm{MHz}$, the interaction rate is expected to be approximately $10^{9} \mathrm{~Hz}$ at the design luminosity. It is not possible to store all the events. For permanent storage, the rate of selected events must be reduced to about $200 \mathrm{~Hz}$. An overall rejection factor of about $10^{7}$ against minimum-bias events must be applied on the one hand, while on the other hand, excellent efficiency must be retained for interesting physics processes with very low expected cross sections, such as the Higgs boson decays or SUSY processes. These are the challenging tasks for the ATLAS trigger and data-acquisition (DAQ) system [39].

In Run 2 the ATLAS detector has a two-level trigger system. The first-level trigger (Level-1 trigger, L1) is implemented in hardware and uses a subset of the detector information to reduce the rate of accepted events from an input rate of up to 40 $\mathrm{MHz}$ to maximally $100 \mathrm{kHz}$. This is followed by a software-based trigger (high-level trigger, HLT) that reduces the rate of recorded events to $1 \mathrm{kHz}$ on average. The event reconstruction at the HLT of objects such as leptons or jets happens only to the extent required by the executed trigger algorithms. Event selections in the HLT are referred to simply as triggers and the collection of all triggers is called the trigger menu.

During Run 2, the LHC delivered a peak luminosity close to $\mathrm{L}=1.7 \times 10^{34} \mathrm{~cm}^{-2} \mathrm{~s}^{-1}$ 
during most of the data-taking period, and briefly exceeded $\mathrm{L}=2.0 \times 10^{34} \mathrm{~cm}^{-2} \mathrm{~s}^{-1}$. The primary triggers that comprise the standard physics trigger menu and their measured rates are shown in Figure 3.7. 


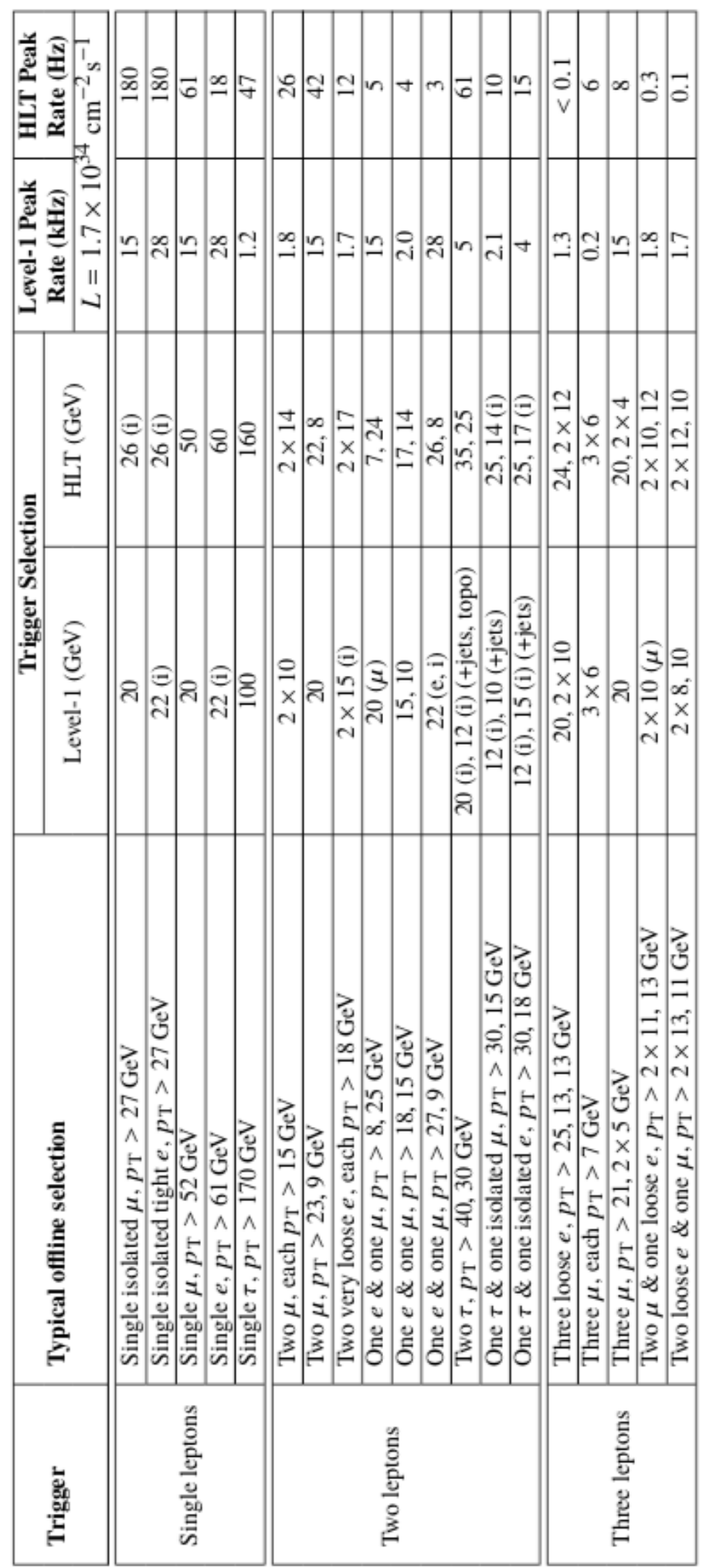

Figure 3.7: The observed rates per trigger are reported at a luminosity of $1.7 \times$ $10^{34} \mathrm{~cm}^{-2} \mathrm{~s}^{-1}$ and $\langle\mu\rangle \simeq 50$ as raw rates, including rate overlapping between triggers [40]. 


\section{CHAPTER IV}

\section{ITk UPGRADE OF THE ATLAS EXPERIMENT}

\subsection{Introduction}

During Run 2 the LHC has been operating at an instantaneous luminosity of $10^{34} \mathrm{~cm}^{-2} \mathrm{~s}^{-1}$. As shown in Figure 4.1, the high luminosity upgrade of the LHC (HLLHC) has been approved to start from 2024 with a goal of increasing the luminosity by a factor of 10 beyond the LHC's design value. This requires an upgrade of the ATLAS detector, known as the Phase II upgrade. Design of the upgraded ATLAS detector is underway to deal with the expected increase in luminosity, which also corresponds to an increase in the average number of interactions per bunch crossing $(\langle\mu\rangle)$, known as pile-up, by a factor of 10 from its present value of 20 up to 200, as shown in Figure 4.2. Design considerations for a new ITk for the ATLAS Phase II upgrade at HL-LHC are:

- radiation damage: to cope with 10 times higher integrated luminosity up to $3000 \mathrm{fb}^{-1}$

- high flux: to cope with 7 times increase in instantaneous luminosity up to $7.5 \times 10^{34} \mathrm{~cm}^{-2} \mathrm{~s}^{-1}$

- high granularity: to cope with 10 times higher pile-up. 


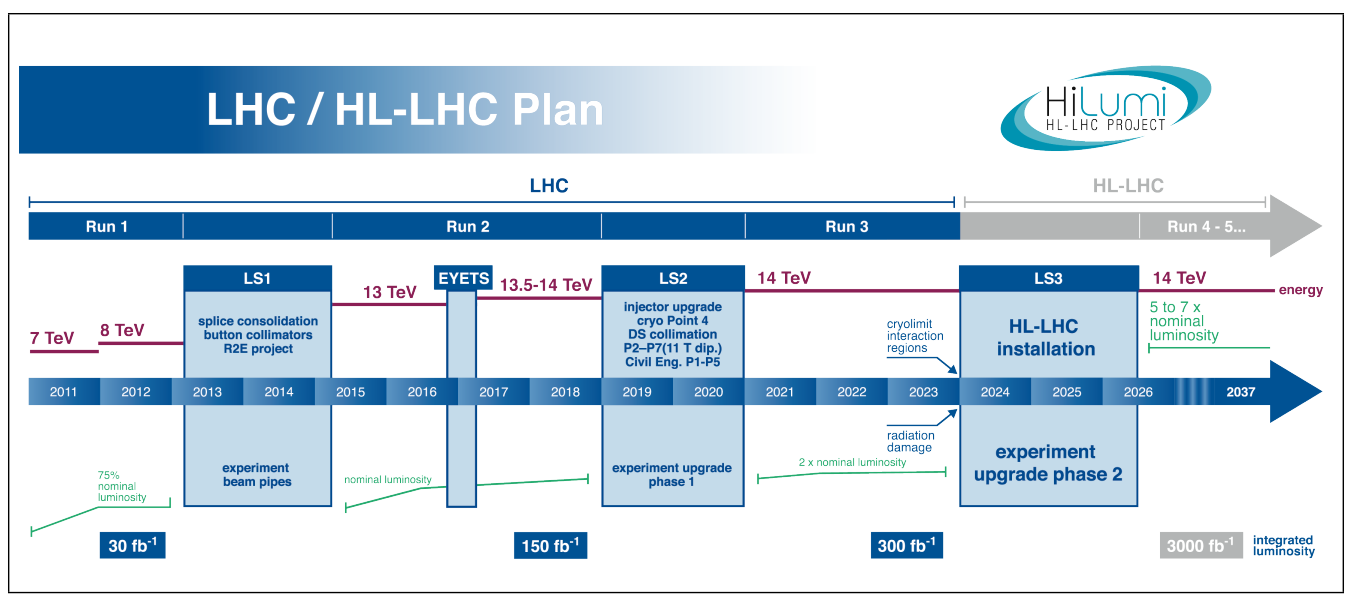

Figure 4.1: Schedule for High Luminosity LHC (HL-LHC) upgrade (Courtesy: Reference [41]).

An all silicon based new Inner Tracker (ITk) with fine granularity and increased fiducial coverage of pseudo-rapidity $(\eta)$ up to 4 units is an integral part of this Phase II upgrade.

Benchmarking of timing studies of track reconstruction in the Inner Detector (ID) used in Run 2 versus the new proposal of upgraded ITK are presented in this chapter [42]. Such studies are critical to the development and optimization of a new detector planned for the Phase II upgrade. In order to have stable results, it is not appropriate to benchmark the CPU requirements in a shared computing environment. These studies were performed in a standalone environment run in a single-user mode after installing the ATLAS software in a new computing environment acquired in 2017 by the Physics Department from a generous gift by an UofL alumni, Sam Lord, consisting of Intel Xeon Phi 1.3 GHz 64-core CPU, 116 GB RAM memory, a 200 GB SSD disk and a 5.5 TB raid array. This study measures the track reconstruction times in terms of HEP-SPEC06 benchmarking units as a function of increasing pile-up for each algorithm of the tracking software. 


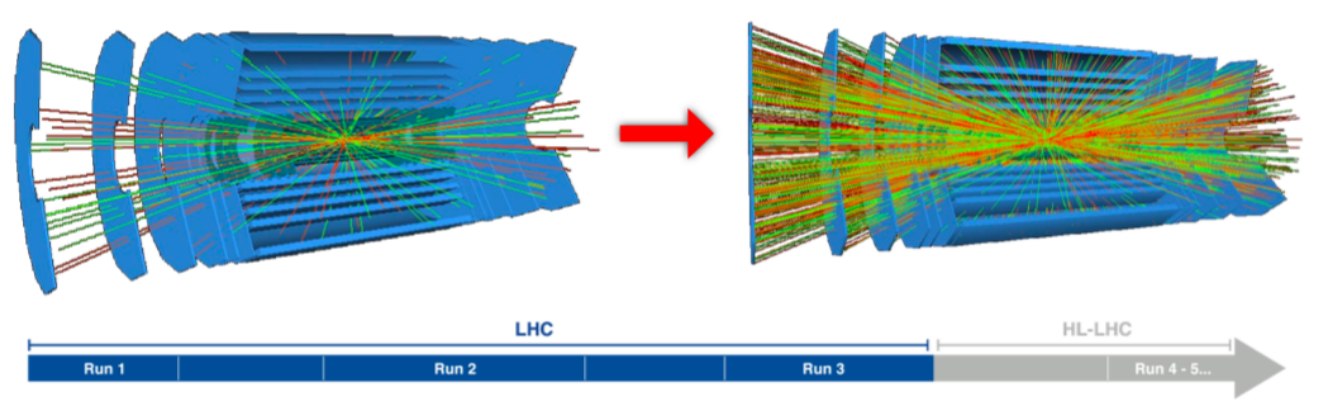

Factor of 10 increase in Pile-Up

(\# of interactions per bunch crossing)
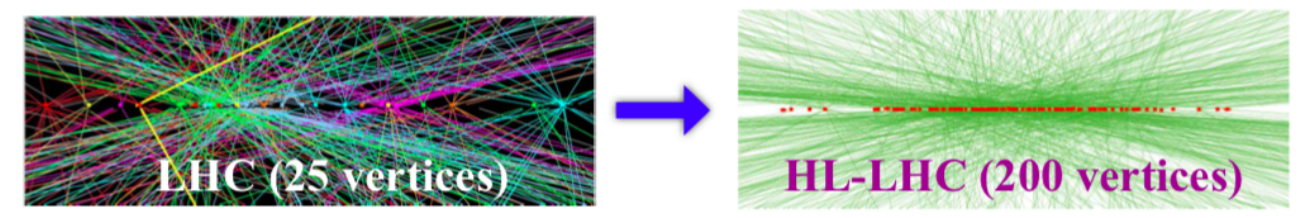

Figure 4.2: More primary vertices seen due to higher pile-up.

\subsection{The ATLAS Inner Tracker(ITk) Layout}

The ITk is an all-silicon detector consisting of a Pixel subsystem with a pseudorapidity coverage of $|\eta|<4.0$ and a Strip subsystem covering the interval $|\eta|<2.7$. The Pixel sub-system consists of five inner flat barrel layers and five layers of inclined or vertical rings providing coverage in the forward region. The Strip subsystem comprises four outer strip module layers in the barrel region and six disks in the end-caps. This combination is designed to have at least 9 precision measurements per track for the full width of the expected beam spot size, which is assumed to have a Gaussian shape with a width of $50 \mathrm{~mm}$, for all charged particles with $p_{\mathrm{T}}>1 \mathrm{GeV}$ traversing through the detector within $|\eta|<4.0$.

A simulated view of the new ITk layout is shown in Figure 4.3. The position of layers in radius $(R)$ versus distance along beam pipe $(z)$ in the ITk layout is compared with the Inner Detector currently being used in Run 2 (ID-Run2) in Figure 4.4. The salient differences are: 


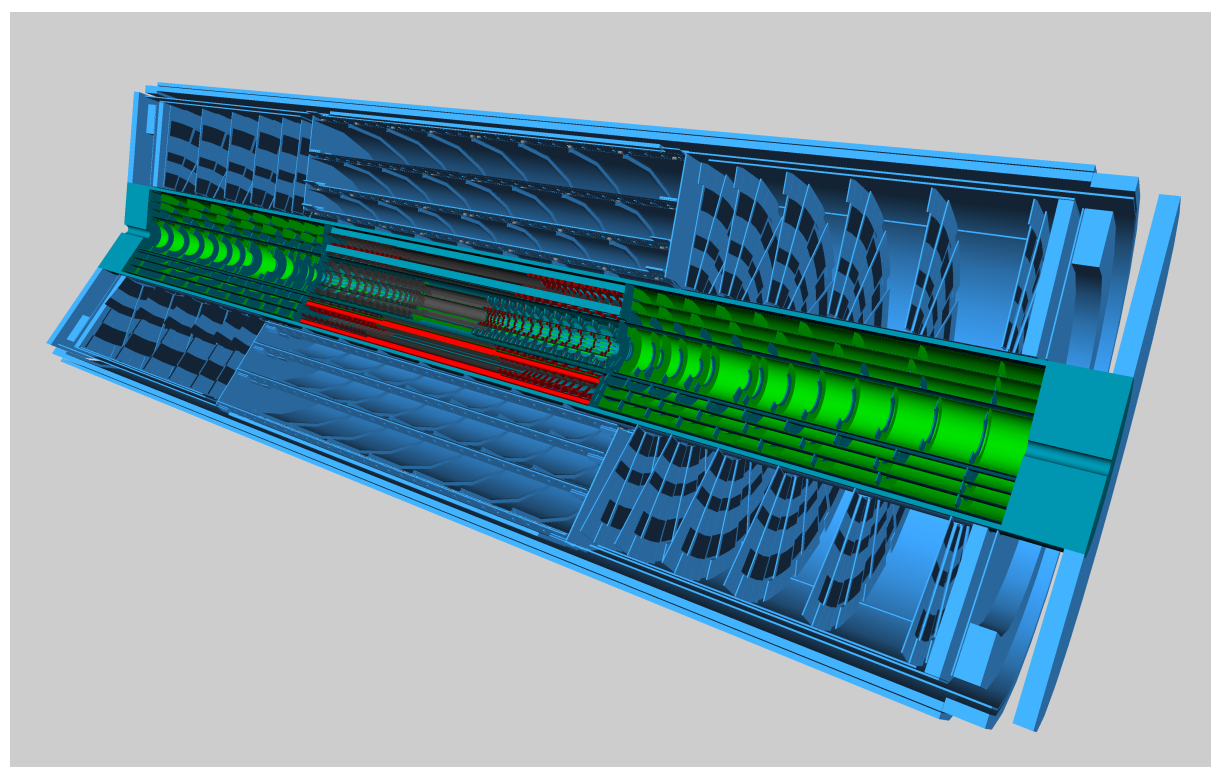

Figure 4.3: 3D split view of the ATLAS Phase-II Inner Tracker ITk with the Inclined Duals detector layout (Courtesy: Reference [43]).

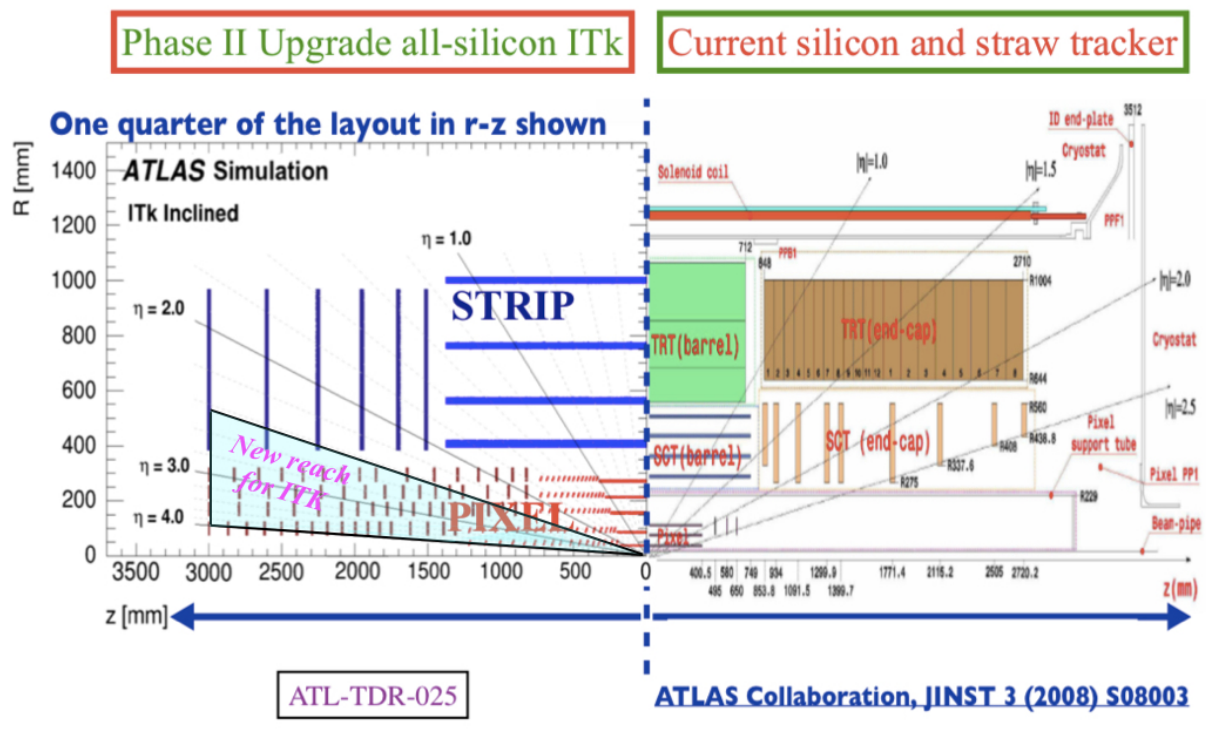

Figure 4.4: $R$ vs. $z$ layout of ITk (left) and ID-Run2 (right). 
- ID-Run2 has 3 sub-components: Pixel, Strip (SCT) and Transition Radiation straw detector (TRT). The all-silicon based ITK will have only the first two sub-components.

- Angular coverage of ID-Run2 is up to $|\eta|<2.5$, whereas the new ITk will extend the coverage up to $|\eta|<4.0$.

- Pixel module size in ID-Run2 is $50 \times 250 \mu m^{2}$ in the innermost layer and $50 \mathrm{x}$ $400 \mu \mathrm{m}^{2}$ for remaining layers, whereas the ITK currently plans to use 50 x 50 $\mu m^{2}$.

- Readout bandwidth for ID-Run2 is about 160 Mbyte/s, whereas for ITk it is expected to be 5 Gbyte/s.

\subsection{Simulation and Track Reconstruction}

ITk simulation helps to choose optimal detector layout. Numerous improvements have been made in the description of the passive material, resulting in a more realistic estimate of the ITk tracking material budget. Figure 4.5 shows the simulated locations of detector material for one quadrant of the detector, while comparisons of the material description of all detector elements used in the simulation for ID-Run2 and ITk are shown in Figure 4.6. The difference of factor of three in the y-axis shows the extent of the optimization of material budget in the new ITk, which is greatly reduced to cope up with the high radiation dose.

\subsubsection{Simulation samples}

The production of simulated samples follows the following steps: Monte Carlo event generation, detector simulation using Geant4, digitisation of simulated energy deposits into the actual detector read-out data format, and event reconstruction starting from the charged tracks seen in the detector. This offline software chain has been 


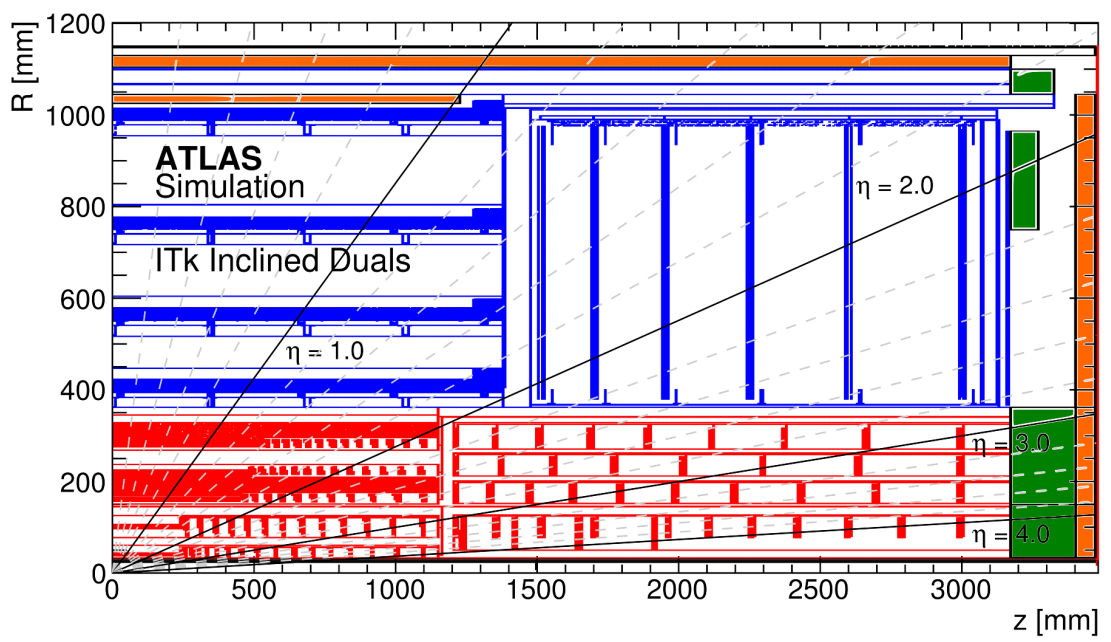

Figure 4.5: Location of the material for one quadrant of the ITk Layout. The Pixel detector is shown in red, and the strip detector in blue (Courtesy: Reference [43]).
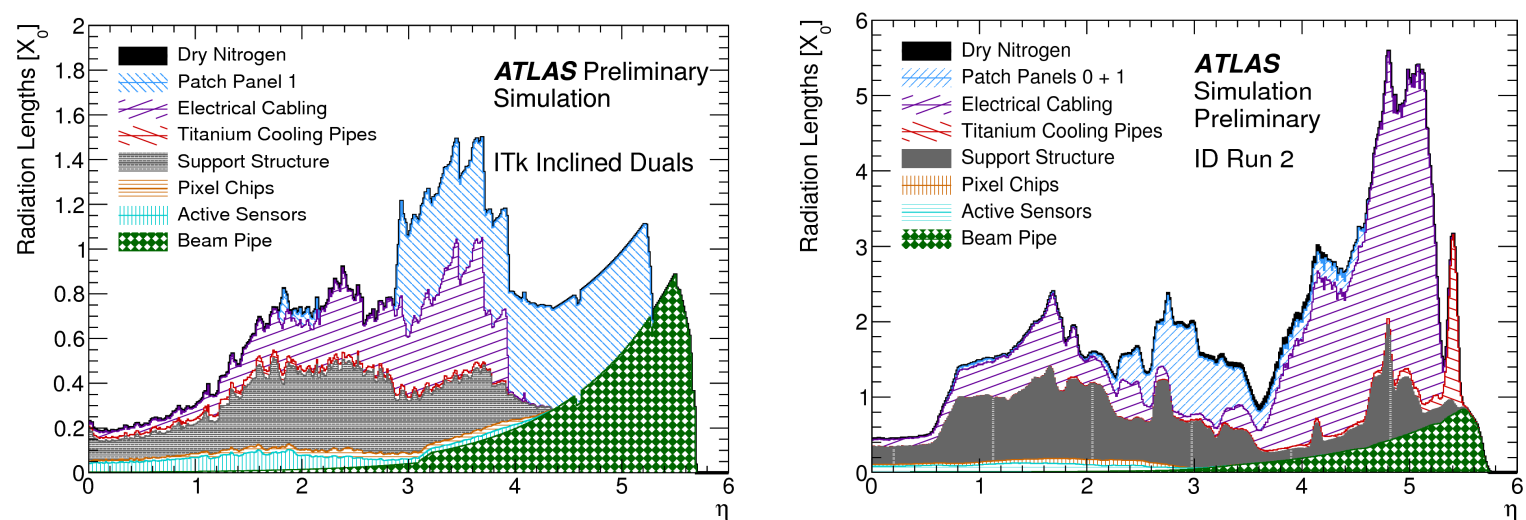

Figure 4.6: Material map of ITk (left) and ID-Run2 (right) vs. $\eta$. 
adapted for the upgrade ITk Detector, including a dedicated tuning of the track reconstruction software.

To benchmark the CPU requirements, $t \bar{t}$ events were generated using PowhegBox $[44,45,46,47,48,49]$ at $\langle\mu\rangle=0,60,140$ and 200 with ITk and at 0,20 and 60 with ID-Run2.

\subsubsection{Track Reconstruction}

The track reconstruction is important as input for the reconstruction of charged particles such as muons, electrons, taus and jets. The track reconstruction is broadly divided into four stages:

- The first step of event reconstruction in ITk is the formation of clusters from individual channels with a hit in the Pixel and Strip detectors, respectively. For the Pixel detector, the cluster formation starts by grouping hits in adjacent pixels to form clusters. Two algorithms are used to determine the cluster position. The first algorithm (digital clustering) only uses the information that a pixel has a hit to determine the cluster position, while the second algorithm (analogue clustering) makes use of the analogue information capabilities of the readout chip to further refine the precision of the cluster position, interpolating the charge measurement of the first and last pixel hit in both directions.

- The next step after the clustering is the space point (SP) formation. Here, the strip cluster information from both sides of a barrel stave or end-cap petal is used to construct strip combinations, exploiting the small stereo angle to obtain precise information. The pixel and strip space points are then used in the seeding stage. Seeds are formed by three space points in the Pixel or Strip detector, labelled as PPP and SSS. For each triplet combination, all space points are required to be on a straight line in $R$ vs. $z$. They form a circle in $R$ vs. $\phi$, 
allowing an estimate of the initial helix parameters. After applying initial $p_{\mathrm{T}}$ and impact parameter cuts, a confirmation of the seed in a $4^{\text {th }}$ layer is made to further reject fake combinations. Finally, seeds are accepted after a duplicate removal.

- The next step is track finding. The track seeding starts with strip space point triplet combinations in the first iteration and then makes use of the pixel information to find additional track candidates. For each accepted seed, a search road is defined, in which a combinatorial Kalman filter is used to find one or more track candidates.

- The last stage is ambiguity resolution stage. The aim of this stage is to reject incomplete and duplicate tracks, resolve situations with shared clusters between several tracks, and remove the fake tracks. This is achieved by scoring the track candidates based on the presence or absence of hits when crossing the sensor layers. Each track candidate considered is fitted using the Global $\chi^{2}$ track fit. The procedure is repeated iteratively, attributing shared clusters to the higher scoring track candidate, and refitting candidates that got modified, to obtain the final set of tracks.

\subsubsection{CPU Timing}

The large multiplicity of tracks in Phase II tracking environment with an average pile-up of up to 200 presents a computing challenge in terms of CPU time required in the reconstruction sequence. Acceptance of seeds used to start the combinatorial Kalman track finder in the third stage and ambiguity resolution in the last stage are two of the most time consuming components in the pattern recognition. From the very beginning of the detector optimization process, the timing performance of each sub-algorithm has been carefully taken into account with an aim of minimiz- 
ing the combinatorial complexity in track reconstruction, and hence the total CPU requirement.

List of the major blocks in the reconstruction sequence used for both ITk and ID-Run2, respectively, are listed below:

- ITk: Total $=$ Si Pre-Processing + SpacePoint + Track Finding + Ambiguity + Vertexing + General + Non-ITk

- ID-Run2: Total $=$ Si Pre-Processing + SpacePoint + Pattern + Ambiguity + Back-tracking + Vertexing + General + Low-beta + Non-ID

The list of the all algorithms for these blocks for both ITk and ID-Run2 are detailed in Tables 4.1 and 4.2 respectively.

\subsubsection{Experimental Procedure}

Reconstruction times should preferably be benchmarked in a controlled computing setup instead of a shared-CPU environment. This study is performed in a single-user mode in the Intel Xenon 7210 machine with $1.3 \mathrm{GHz}, 64$ core CPU, 116 GB RAM, where a dedicated scheduler is used to run the computing jobs without external interference or interruptions. The traditional way to present CPU times use a standard HEP-Spec06 benchmarking suite. Accordingly, the reconstructed CPU times reported here are multiplied by a HS06 factor of 3.5, which has been obtained by separately benchmarking our cluster.

The procedure is:

- reconstruction jobs are split into 20 sets of 50 events each.

- Time taken by $1^{\text {st }}$ event in each set is averaged over 5 separate jobs.

- Averaged initialization time used by the $1^{\text {st }}$ event is subtracted to get total time used by the remaining 49 events in each set. 


\begin{tabular}{|c|c|}
\hline Detector & ITk \\
\hline Si Pre-Processing & $\begin{array}{c}\text { InDetSCT_Clusterization + } \\
\text { InDetPixelClusterization }\end{array}$ \\
\hline SP Formation & InDetSiTrackerSpacePointFinder \\
\hline Track Finding & InDetSiSpTrackFinderSLHC \\
\hline Ambiguity Solution & InDetAmbiguitySolverSLHC \\
\hline Primary Vertex & InDetPriVxFinder \\
\hline Non ITk & MuonCombinedInDetCandidateAlg \\
\hline ITk General & $\begin{array}{c}\text { InDetCaloClusterROISelector } \\
\text { InDetCopyAlg } \\
\text { InDetTrackCollectionMerger } \\
\text { InDetTrackParticles } \\
\text { InDetVxLinkSetter } \\
\text { InDetRecStatistics }\end{array}$ \\
\hline
\end{tabular}

Table 4.1: All algorithms for track reconstruction in the full detector with ITk layout. 


\begin{tabular}{|c|c|}
\hline Detector & ID-Run2 \\
\hline Si Pre-Processing & $\begin{array}{c}\text { InDetSCT_Clusterization + } \\
\text { InDetPixelClusterization }\end{array}$ \\
\hline SP Formation & InDetSiTrackerSpacePointFinder \\
\hline Track Finding & $\begin{array}{c}\text { InDetSiSpTrackFinderSLHC + } \\
\text { InDetSiSpTrackFinderForwardTracks }+ \\
\text { InDetTRT_Extension }+ \\
\text { InDetTRT_SeededTrackFinder }\end{array}$ \\
\hline Ambiguity Solution & $\begin{array}{c}\text { InDetAmbiguitySolver }+ \\
\text { InDetAmbiguitySolverForwardTracks }+ \\
\text { InDetExtensionProcessor }+ \\
\text { InDetTRT_SeededAmbiguitySolver }\end{array}$ \\
\hline Back-Tracking & $\begin{array}{c}\text { InDetTRT_RIO_Maker + } \\
\text { InDetTRT_TrackSegmentsFinder + } \\
\text { InDetTRT_StandaloneTrackFinder }\end{array}$ \\
\hline Primary Vertex & InDetPriVxFinder \\
\hline Non ID & MuonCombinedInDetCandidateAlg \\
\hline ID General & $\begin{array}{c}\text { InDetCaloClusterROISelector } \\
\text { InDetSegmentPRD_Association } \\
\text { InDetTRTonly_PRD_Association } \\
\text { InDetPRD_AssociationForwardTracks } \\
\text { InDetTrackCollectionMerger } \\
\text { InDetTrackSlimmer } \\
\text { InDetTrackParticles } \\
\text { InDetForwardTrackParticles } \\
\text { InDetVxLinkSetter } \\
\text { InDetBCM_ZeroSuppression } \\
\text { InDetCosmicsEventPhase } \\
\text { InDetRecStatistics }\end{array}$ \\
\hline
\end{tabular}

Table 4.2: All algorithms for track reconstruction in the full detector with ID-Run2 layout. 
- Gaussian fit is performed to the distribution of (time/event) over each of the 20 sets.

- Final result is quoted as (fitted mean \pm standard deviation $/ \sqrt{20}$ ).

Gaussian fit to distribution of time/event (seconds) over 20 sets in various pileup values for adding all individual algorithm for ITk and ID-Run2 are shown in Figures 4.7 and 4.8 , respectively.

One crucial requirement on the track reconstruction software chain is that the total processing time per event must be minimised, to stay within the limited computing time budget available for event reconstruction even in the presence of an average of 200 pileup collisions per event. Results of benchmarking the full Detector for ITk in software release 20.20.12.3 for step 3 using analogue clustering with pitch size 50x50 $\mu m^{2}$ are shown in Table 4.3, and for ID-Run2 are shown in Table 4.4.

Due to algorithmic improvements and the move to a full silicon tracker, even with the current version of the ITk software, the CPU time taken up by track reconstruction at $\langle\mu\rangle=200$ is expected to be smaller than the corresponding number at $\langle\mu\rangle=60$ for the present detector, as shown in the summary plot Figure 4.9 [42]. 
for $\langle\mu\rangle=0$ (left) and 60 (right)
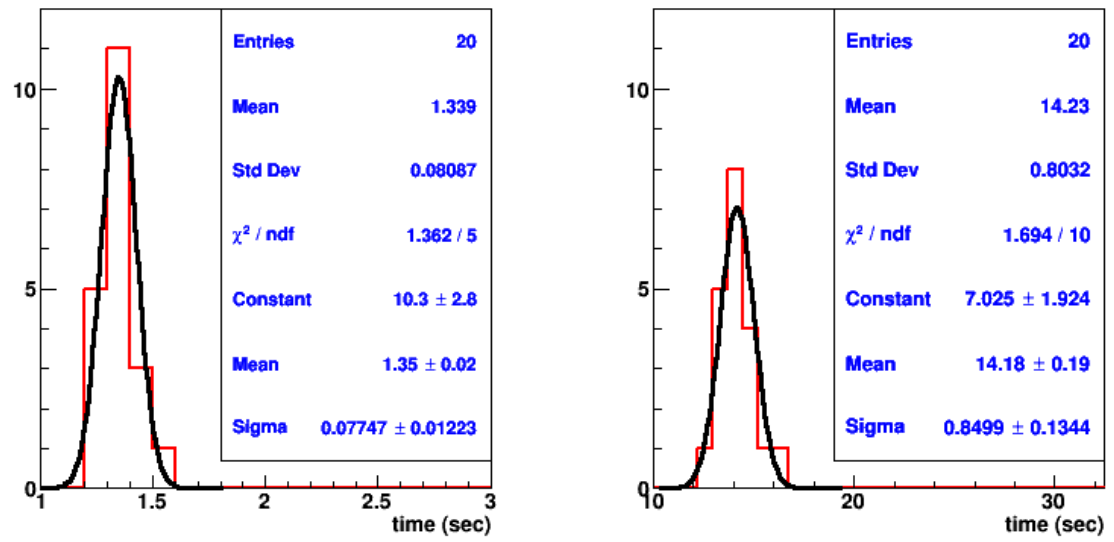

for $\langle\mu\rangle=140$ (left) and 200 (right)
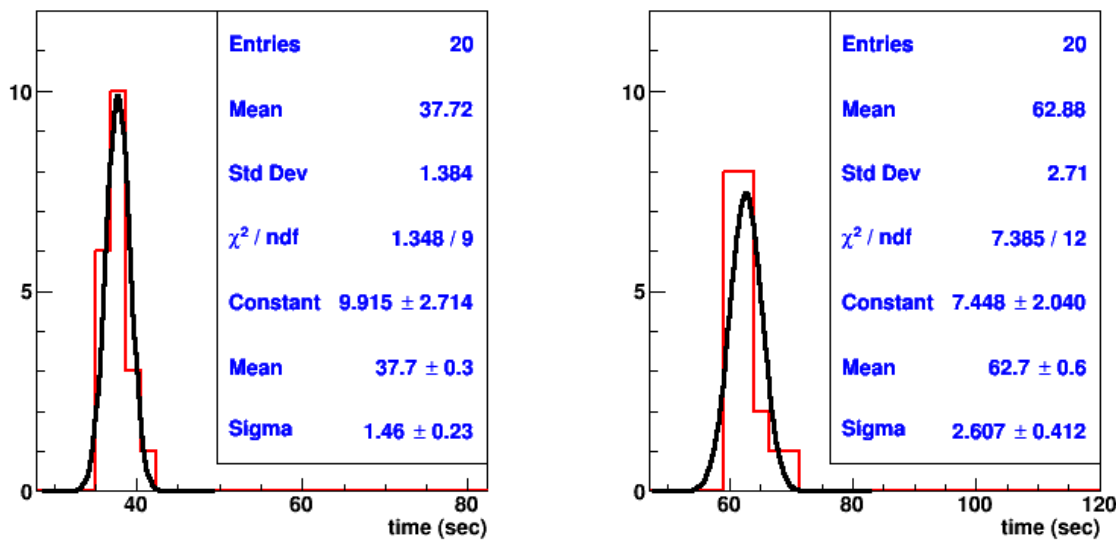

Figure 4.7: Reconstruction times studied from RAWtoESD output in analoge clustering for ITk studies for all Algorithms.

for $\langle\mu\rangle=20$ (left) and 60 (right)
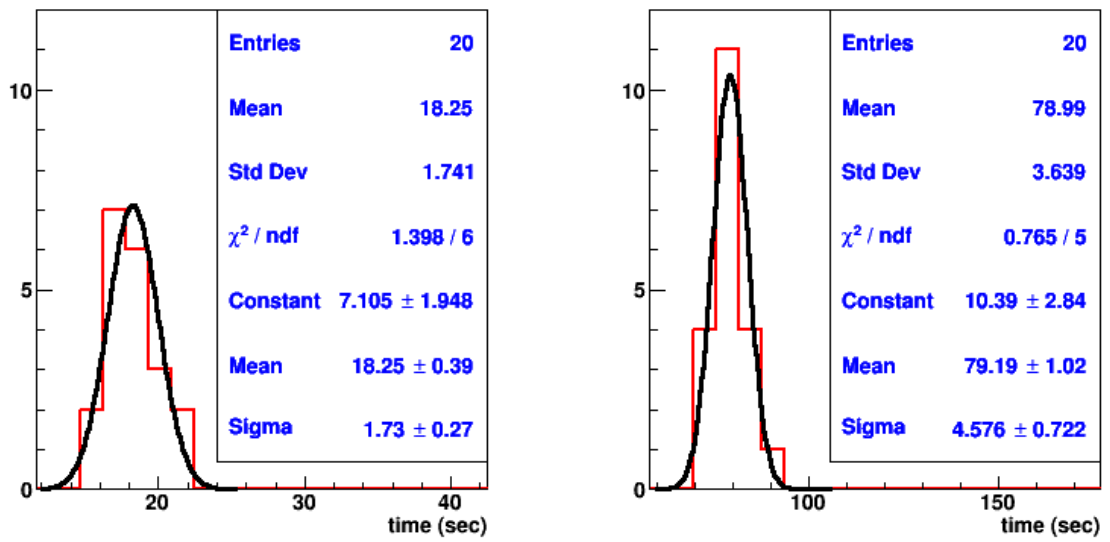

Figure 4.8: Reconstruction times studied from RAWtoESD output ID-Run2 for all algorithms. 


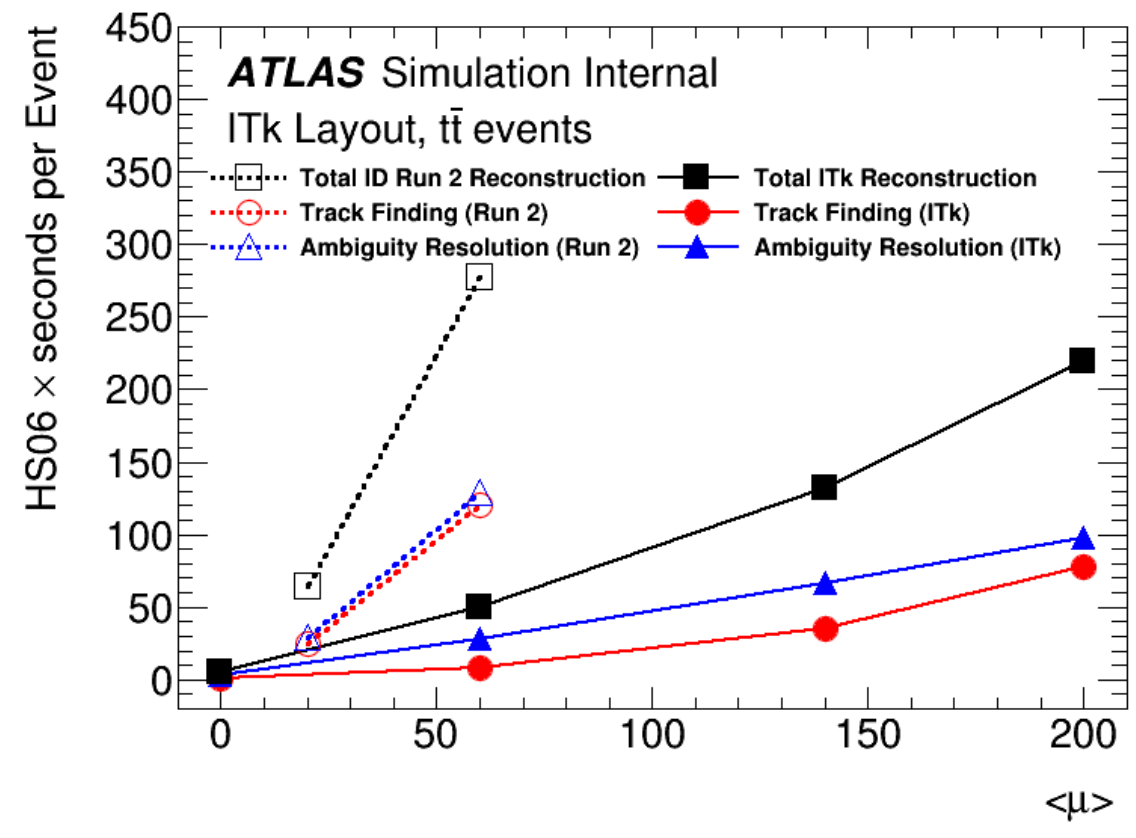

Figure 4.9: The total CPU time in HS06 x seconds required to reconstruct a $t \bar{t}$ event as well as the CPU time needed for the silicon track finding and for the ambiguity resolution steps as a function of $\langle\mu\rangle$ for the ITk Layout with a $50 \times 50 \mu \mathrm{m}^{2}$ pixel pitch. For comparison the corresponding performance for the current ID-Run2 detector track reconstruction is shown for an average of 20 and 60 pileup events. 


\begin{tabular}{|c|c|c|c|c|}
\hline 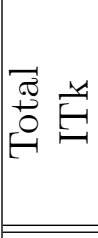 & 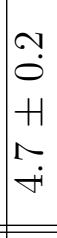 & $\begin{array}{l}\tilde{N} \\
\sim \\
H \\
H \\
0 \\
\dot{H} \\
\mathscr{H}\end{array}$ & 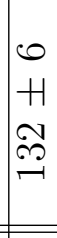 & $\begin{array}{l}0 \\
-1 \\
+1 \\
0 \\
\stackrel{-}{\sim}\end{array}$ \\
\hline 党 & $\begin{array}{l}20 \\
8 \\
8 \\
0 \\
0 \\
H \\
+1 \\
0 \\
0 \\
\dot{0}\end{array}$ & $\begin{array}{l}\not \\
\delta \\
0 \\
0 \\
H \\
+ \\
\dot{0}\end{array}$ & $\begin{array}{l}\sigma \\
0 \\
0 \\
H \\
+1 \\
0\end{array}$ & $\begin{array}{l}\overrightarrow{0} \\
\dot{0} \\
H \\
+ \\
0 \\
0\end{array}$ \\
\hline 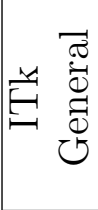 & $\begin{array}{l}\sigma \\
0 \\
0 \\
H \\
\infty \\
0\end{array}$ & $\begin{array}{l}\breve{0} \\
0 \\
H \\
0 \\
\dot{v}\end{array}$ & $\begin{array}{l}? \\
0 \\
H \\
H \\
0 \\
0\end{array}$ & $\begin{array}{l}\vec{H} \\
0 \\
+1 \\
0\end{array}$ \\
\hline 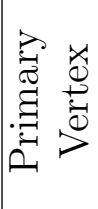 & $\begin{array}{l}0 \\
0 \\
0 \\
H \\
1 \\
0 \\
0\end{array}$ & $\begin{array}{l}- \\
0 \\
H \\
\sim\end{array}$ & $\begin{array}{l}0 \\
0 \\
H \\
+1 \\
\infty \\
\dot{H}\end{array}$ & $\begin{array}{l}\dddot{0} \\
\dot{\theta} \\
0\end{array}$ \\
\hline 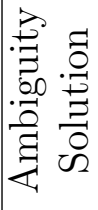 & $\begin{array}{l}\breve{b} \\
0 \\
H \\
0 \\
0 \\
ن\end{array}$ & $\begin{array}{l}\stackrel{\sim}{\sim} \\
\stackrel{-}{H} \\
\infty \\
\sim \\
\sim\end{array}$ & $\begin{array}{l}\infty \\
H \\
0 \\
0\end{array}$ & $\begin{array}{l}\sim \\
+ \\
H \\
N \\
\hat{\sigma}\end{array}$ \\
\hline 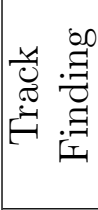 & $\begin{array}{l}m \\
0 \\
0 \\
H \\
0 \\
0\end{array}$ & $\begin{array}{l}\vec{H} \\
0 \\
H \\
1 \\
\infty \\
\infty\end{array}$ & 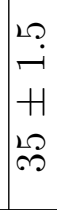 & $\begin{array}{l}20 \\
\infty \\
+1 \\
\infty \\
1\end{array}$ \\
\hline कि & $\begin{array}{l}\overrightarrow{0} \\
\dot{0} \\
+1 \\
20 \\
\stackrel{0}{0}\end{array}$ & $\begin{array}{l}\overrightarrow{-} \\
0 \\
H \\
-1 \\
-1\end{array}$ & 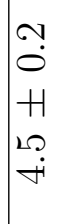 & $\begin{array}{l}3 \\
0 \\
+1 \\
+ \\
0\end{array}$ \\
\hline 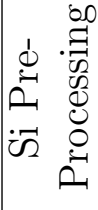 & $\begin{array}{l}\stackrel{2}{0} \\
\dot{0} \\
H \\
1 \\
0 \\
0\end{array}$ & $\begin{array}{l}\dddot{m} \\
0 \\
H \\
\sim \\
0\end{array}$ & $\begin{array}{l}\sim \\
0 \\
H \\
0 \\
-1\end{array}$ & $\begin{array}{l}\vec{H} \\
H \\
\vec{H} \\
\stackrel{2}{\sim}\end{array}$ \\
\hline$\stackrel{\varrho}{\oplus}$ & 0 & 8 & $\stackrel{ }{\stackrel{9}{1}}$ & 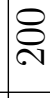 \\
\hline 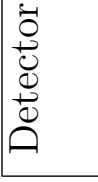 & 至 & 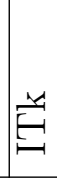 & $\underline{E}$ & 㟧 \\
\hline
\end{tabular}




\begin{tabular}{|c|c|c|}
\hline 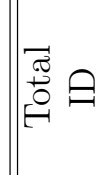 & $\begin{array}{l}\infty \\
+1 \\
\overrightarrow{0}\end{array}$ & $\begin{array}{l}\stackrel{\sim}{\sim} \\
+ \\
\stackrel{N}{N} \\
\stackrel{N}{N}\end{array}$ \\
\hline$\theta$ & $\begin{array}{l}2 \\
\tilde{8} \\
\dot{0} \\
+1 \\
20 \\
0 \\
0 \\
0\end{array}$ & $\begin{array}{l}5 \\
0 \\
0 \\
H \\
10 \\
\stackrel{2}{0} \\
0\end{array}$ \\
\hline 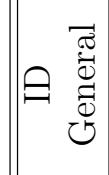 & 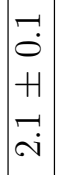 & $\begin{array}{l}+4 \\
0 \\
+1 \\
\infty \\
\infty \\
\infty\end{array}$ \\
\hline 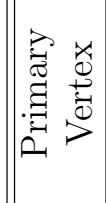 & 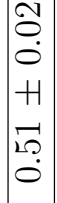 & $\begin{array}{l}-1 \\
0 \\
+1 \\
\infty\end{array}$ \\
\hline 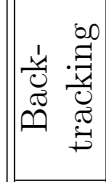 & 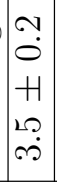 & $\begin{array}{l}\infty \\
0 \\
+1 \\
\infty \\
0 \\
0\end{array}$ \\
\hline 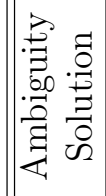 & $\begin{array}{l}\hat{\imath} \\
\dot{0} \\
+1 \\
\stackrel{2}{\Omega}\end{array}$ & \begin{tabular}{l}
0 \\
$H$ \\
2 \\
\multirow{2}{*}{} \\
-1
\end{tabular} \\
\hline 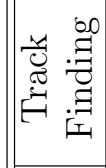 & $\begin{array}{l}\stackrel{\sim}{\sim} \\
\stackrel{-}{H} \\
\stackrel{2}{\sim} \\
\stackrel{2}{\sim}\end{array}$ & \begin{tabular}{l}
20 \\
+1 \\
0 \\
\multirow{2}{1}{} \\
\end{tabular} \\
\hline 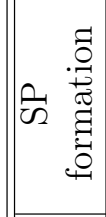 & $\begin{array}{l}\dddot{2} \\
\dot{0} \\
+1 \\
\tilde{R} \\
\dot{0} \\
\end{array}$ & \begin{tabular}{l}
28 \\
0 \\
0 \\
+1 \\
\multirow{2}{*}{} \\
-1 \\
\end{tabular} \\
\hline 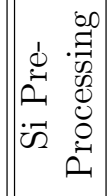 & $\begin{array}{l}-1 \\
0 \\
+1 \\
20 \\
-1 \\
-i\end{array}$ & $\begin{array}{l}10 \\
\stackrel{1}{0} \\
+1 \\
0 \\
0 \\
0\end{array}$ \\
\hline 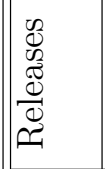 & 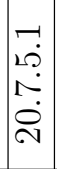 & 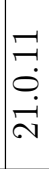 \\
\hline 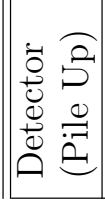 & 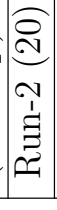 & 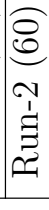 \\
\hline
\end{tabular}




\section{CHAPTER V}

\section{BELLL II DETECTOR}

\section{$5.1 \quad$ Introduction}

Modern electron-positron colliders constitute an effective tool for discovery science, as demonstrated by the important role played by the Belle and BABAR experiments in the citation of 2008 Nobel Prize in Physics. These electron-positron annihilations experiments, popularly known as $B$ factories, also serve as $\tau$ factories, thanks to the large and comparable cross-sections for the production of $B$ and $\tau$ pair events of $1.110 \pm 0.008 \mathrm{nb}$ and $0.919 \pm 0.003 \mathrm{nb}$, respectively, at a center-of-mass (CM) energy of $10.58 \mathrm{GeV}[50]$. While the above two experiments recorded collisions at an instantaneous luminosity of $10^{34} \mathrm{~cm}^{-2} \mathrm{~s}^{-1}$ delivered by the KEKB and PEP-II colliders, the next generation SuperKEKB collider in Japan has now been designed to go up by almost two orders of magnitude, delivering a peak luminosity of $8 \times 10^{35} \mathrm{~cm}^{-2} \mathrm{~s}^{-1}$.

The Belle II experiment located at this worlds highest luminosity accelerator started its data-taking with the full detector configuration in March 2019 as the lone successor to the previous generation of prominent $B$ and $\tau$ factory experiments. With an ultimate goal of recording $50 \mathrm{ab}^{-1}$ of data over the next few years, which will be a factor of 50 and 100 more than Belle and BABAR, respectively, the Belle II experiment will record the worlds largest sample of $10^{11}$ tau-pair events in a pristine 
environment and will play a significant role in moving the field forward.

Compared to its precursor experiment, the Belle II detector operates at 40 times higher event rates, while the backgrounds rates are higher by a factor of 10 to 20 [51]. In order to maintain excellent performance of the detector, the critical issues are to mitigate the effects of higher background levels, which lead to an increase in occupancy and radiation damage, as well as higher fake hits and large pile-up noise in the electromagnetic calorimeter, and to larger amounts from neutron induced backgrounds hits in the muon detector. Also, hadron identification needs to be improved, and uniform hermeticity is required at least as good as in the original Belle detector.

\subsection{Belle II detector}

While the new detector fits the same shell as its predecessor along with the superconducting soleniod magnet and the iron return yoke, all components of the Belle II detector are either new or considerably upgraded [51]. The complete layout of the new and upgraded Belle II detector is shown in Figure 5.1. The design parameters of the Belle II experiment are summarised in Table 5.1, Some critical components are discussed below in some detail.

\subsubsection{Vertex detector (VXD)}

The new vertex detector comprises of two layers of the silicon Pixel Detector (PXD) and and four layers of Silicon Vertex Detector (SVD). These six layers surround a $2 \mathrm{~cm}$ diameter Berrylium beam pipe, as shown in Figure 5.2). The two layers of PXD with 8 and 12 ladders with 40 sensors in total are placed at radii of $r=14 \mathrm{~mm}$ and $r=22 \mathrm{~mm}$ and made up of pixelated sensors of the DEPFET type [52, 53]. The four layers of SVD are placed at radii of $r=38 \mathrm{~mm}, 80 \mathrm{~mm}, 115 \mathrm{~mm}$, and $140 \mathrm{~mm}$ and are equipped with double-sided silicon microstrip sensors.

Currently Belle II is operating in the "Phase 3 commissoning period" from March 


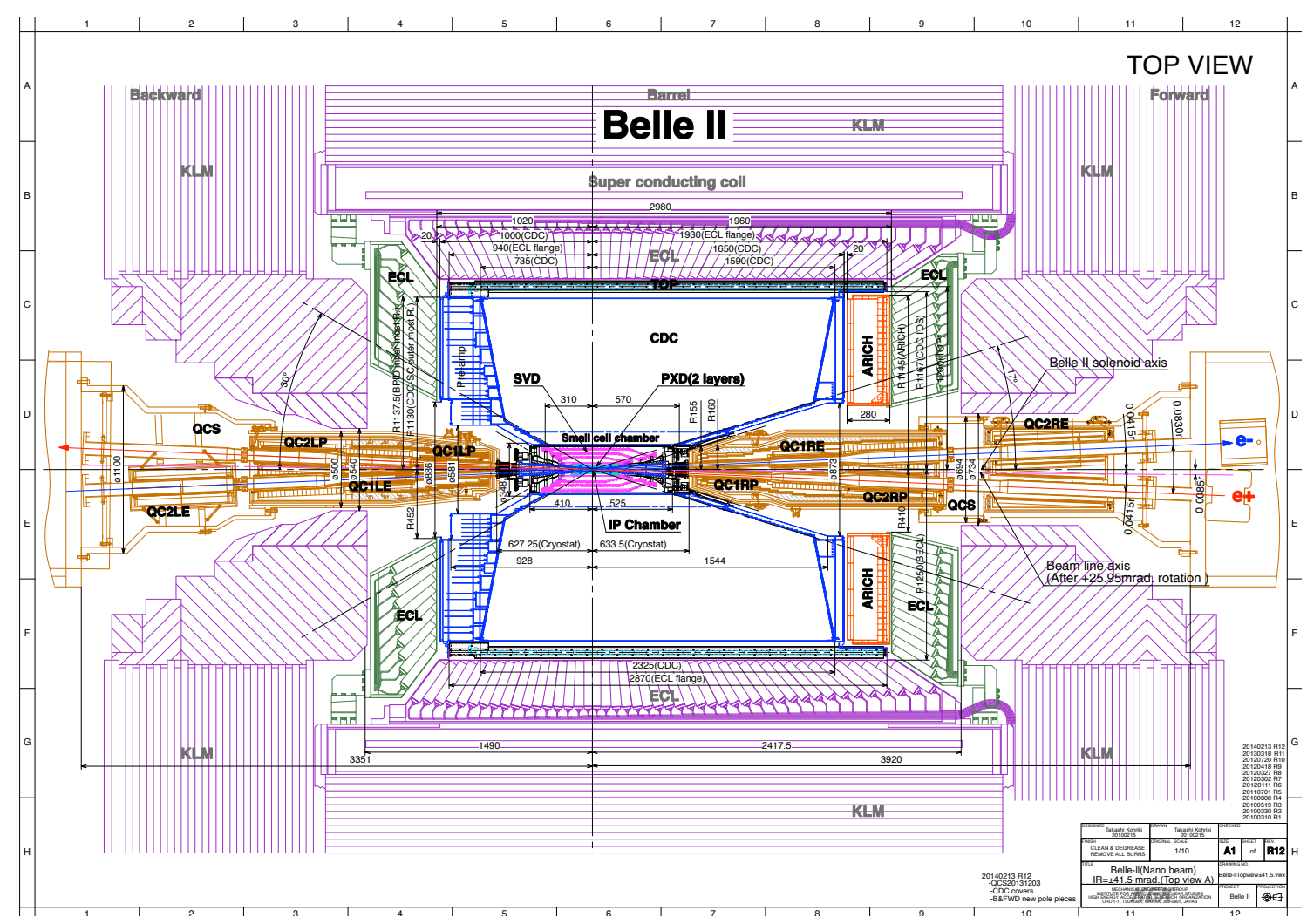

Figure 5.1: Belle II top view (Courtesy: Reference [50]). 


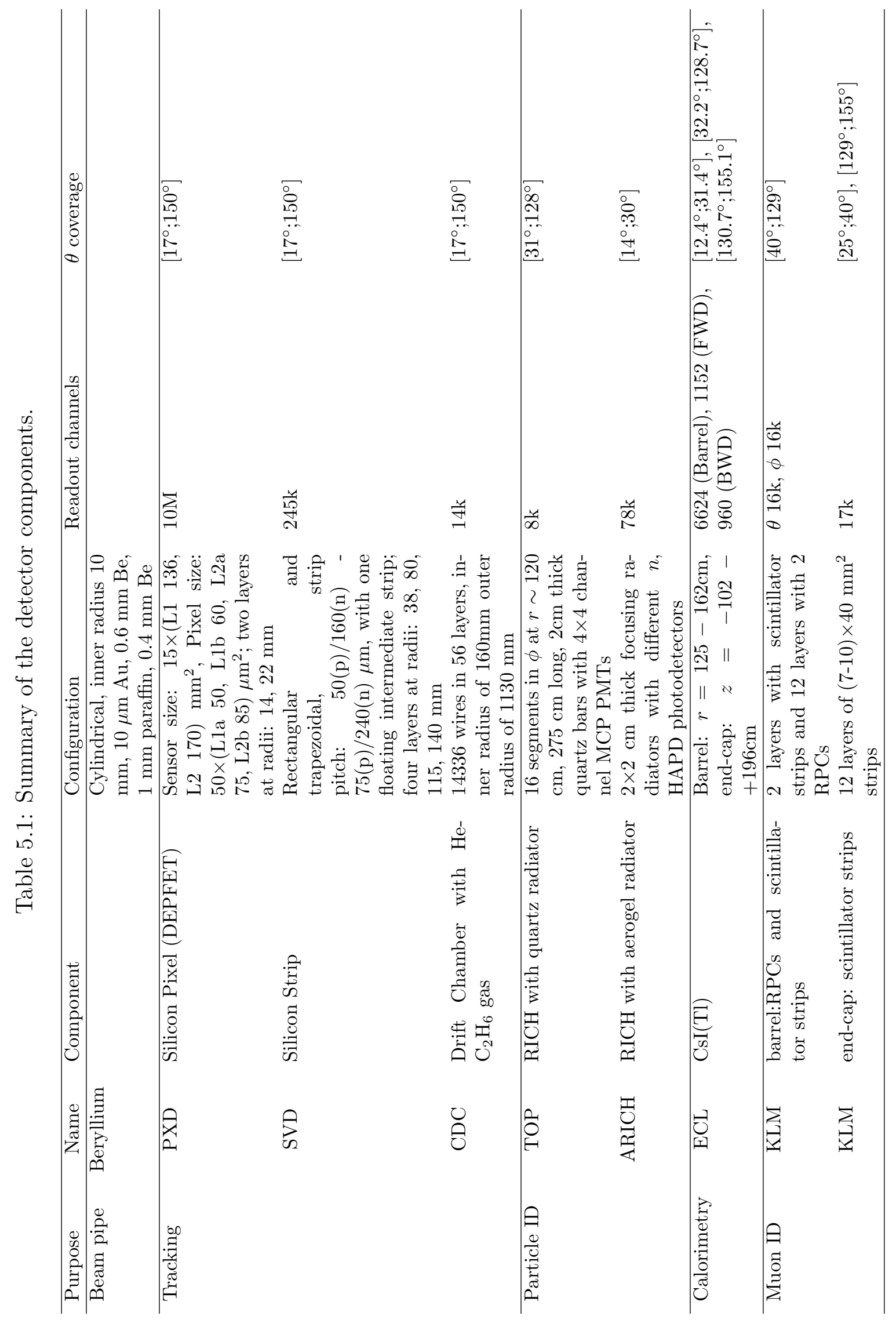


2019 where the installation of PXD has full 8 ladders in the first layer and 2 ladders with 20 sensors in total. Complete installation is planned during a long shutdown in 2022.

The summary table (Table 5.1) lists the sensor strip pitch sizes of the Belle II detector. In comparison, for the Belle experiment the beam pipe was at $15 \mathrm{~mm}$, the innermost layer was at $20 \mathrm{~mm}$ and the outermost layer of a 4 layer vertex detector layer was at a radius of $88 \mathrm{~mm}$. Compared to the Belle vertex detector, the beam pipe and the first two detector layers are closer to the interaction point, and the outermost layer is at a considerably larger radius. Significant improvement in the vertex resolution is expected with respect to Belle as a result of these updates. The reconstruction efficiency for $K_{S}^{0} \rightarrow \pi^{+} \pi^{-}$decays from hits in the vertex detector is expected to improve as well [51].
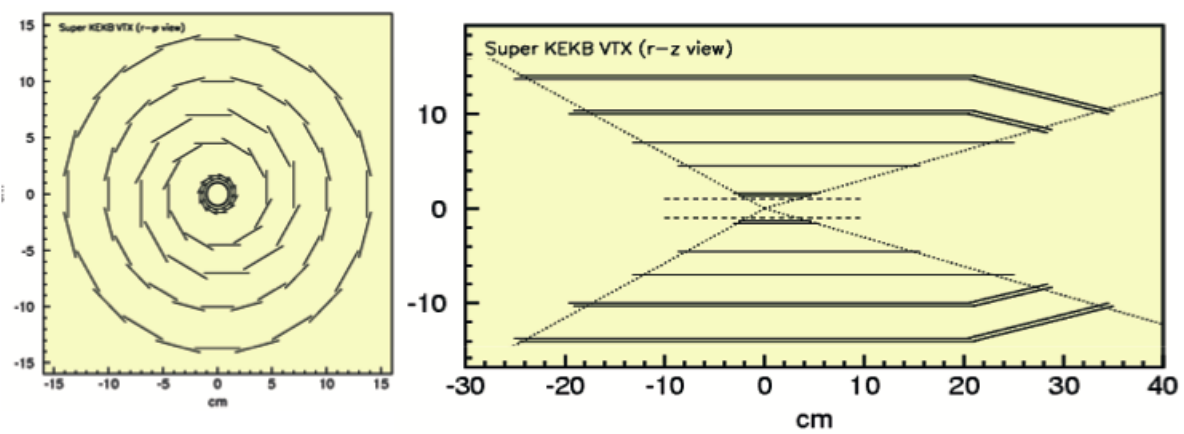

Figure 5.2: A schematic view of the Belle II vertex detector with a Be beam pipe, two pixelated layers and four layers with silicon strip sensors (Courtesy: Reference [50]).

\subsubsection{Central Drift Chamber (CDC)}

A central tracking device with a large volume drift chamber with small drift cells known as the CDC is one of the core instruments of the Belle II spectrometer. Due to the upgrade to a much thinner particle identification device in the barrel region, the CDC extends to a larger radius of $1130 \mathrm{~mm}$ in Belle II as compared to $880 \mathrm{~mm}$ in Belle. The CDC has smaller drift cells than the one used in Belle in order to be 


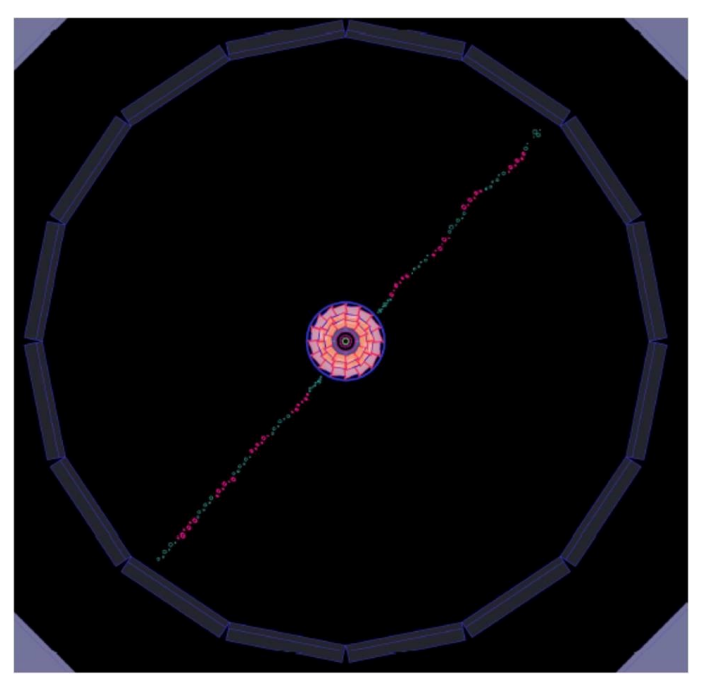

Figure 5.3: A cosmic muon as recorded by the Belle II Central Drift Chamber (CDC).

able to operate at high event rates with increased background levels in Belle II. In total, the CDC contains 14336 sense wires arranged in 56 layers, either in "axial" orientation, e.g. aligned with the solenoidal magnetic field, or in "stereo" orientation, e.g. skewed with respect to the axial wires. By combining information from axial and stereo layers, a full 3D helix track can be reconstructed. The gas chamber in CDC is filled with a $\mathrm{He}-\mathrm{C}_{2} \mathrm{H}_{6} 50: 50$ mixture, which has an average drift velocity of $3.3 \mathrm{~cm} / \mu \mathrm{s}$ and a maximum drift time of about $350 \mathrm{~ns}$ for $17 \mathrm{~mm}$ cell size.

The fully constructed drift chamber has been commissioned with cosmic rays in the Belle II detector. Figure 5.3 shows one such cosmic ray passing through the CDC.

\subsubsection{Particle identification system (TOP and ARICH)}

Particle Identification (PID) consists of a time-of-propagation (TOP) counter in the barrel region $[54,55]$ and a proximity focusing Cherenkov ring imaging detector with aerogel as Cherenkov radiator $(\mathrm{ARICH})$ in the forward end-cap region. The design requirements include good separation of pions and kaons up to about $4 \mathrm{GeV} / c$.

The TOP detector is comprised of 16 modules, as one shown in Figure 5.4. Each module is composed of four parts glued together: two fused silica bars of dimensions 


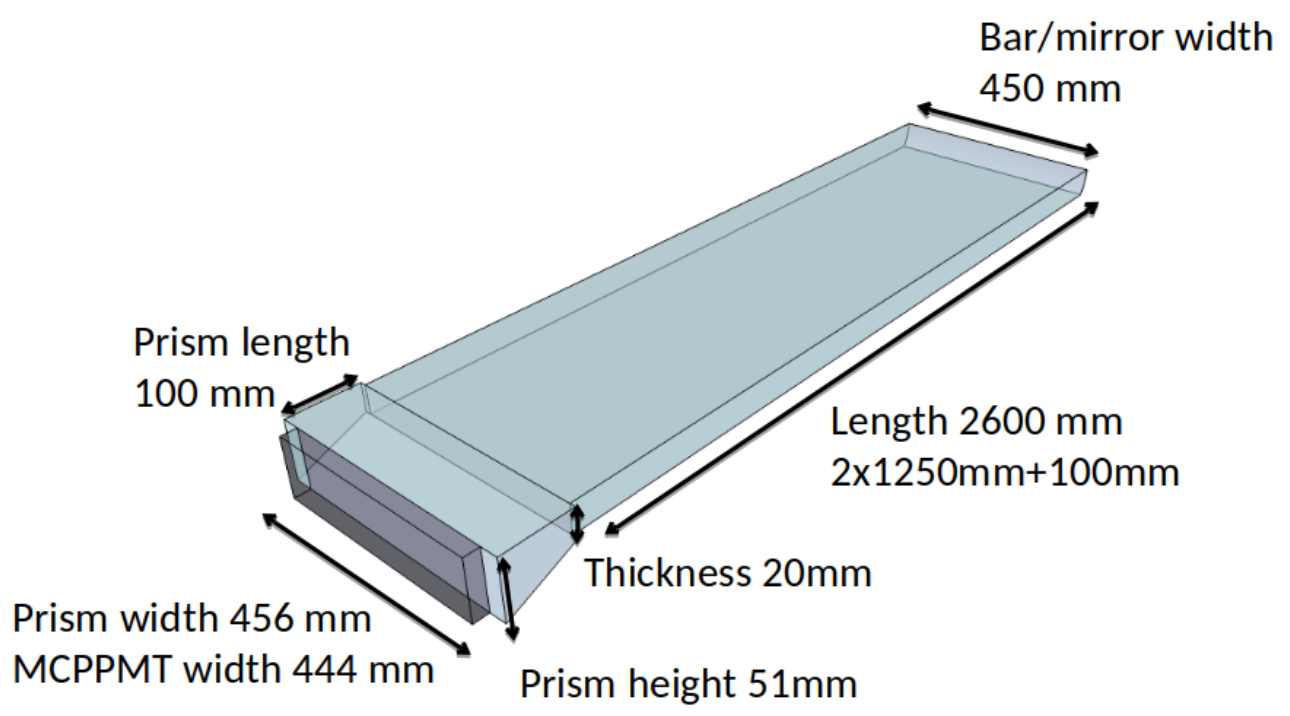

Figure 5.4: One of the 16 modules of the TOP detector (Courtesy: Reference [50]).

$(125 \times 45 \times 2) \mathrm{cm}$ acting as Cherenkov radiator, a mirror located at the forward end of the bars, and a $10 \mathrm{~cm}$ long prism that couples the bar with an array of micro-channelplate photo-multiplier tubes (MCP-PMT) [55, 56]. The TOP detector utilizes total internal reflection of Cherenkov photons produced in a quartz radiator and measures the position and precise arrival time of the propagated photons at the radiator ends. The effect of the mirror is to focus parallel rays of photons into a single pixel of a photo-sensor while the chromatically dispersed rays are detected by separate channels instead of a single channel. For such identification to work, the particle production time has to be known with a precision of about 50 ps, which is indeed challenging, but has already been achieved for the time-of-flight (TOF) counter of Belle [57].

The ARICH detector uses 2 layers of tiles of aerogel for the particle to transition through and a photon detector on the opposite side to measure the Cherenkov radiation. Each layer has a different level of refractive index, given by: $n_{1}=1.045$ and $n_{2}=1.55$, respectively. As shown in Figure 5.5, a hybrid avalanche photon detector (HAPD) is used for the single photon sensitive high granularity sensor, developed 


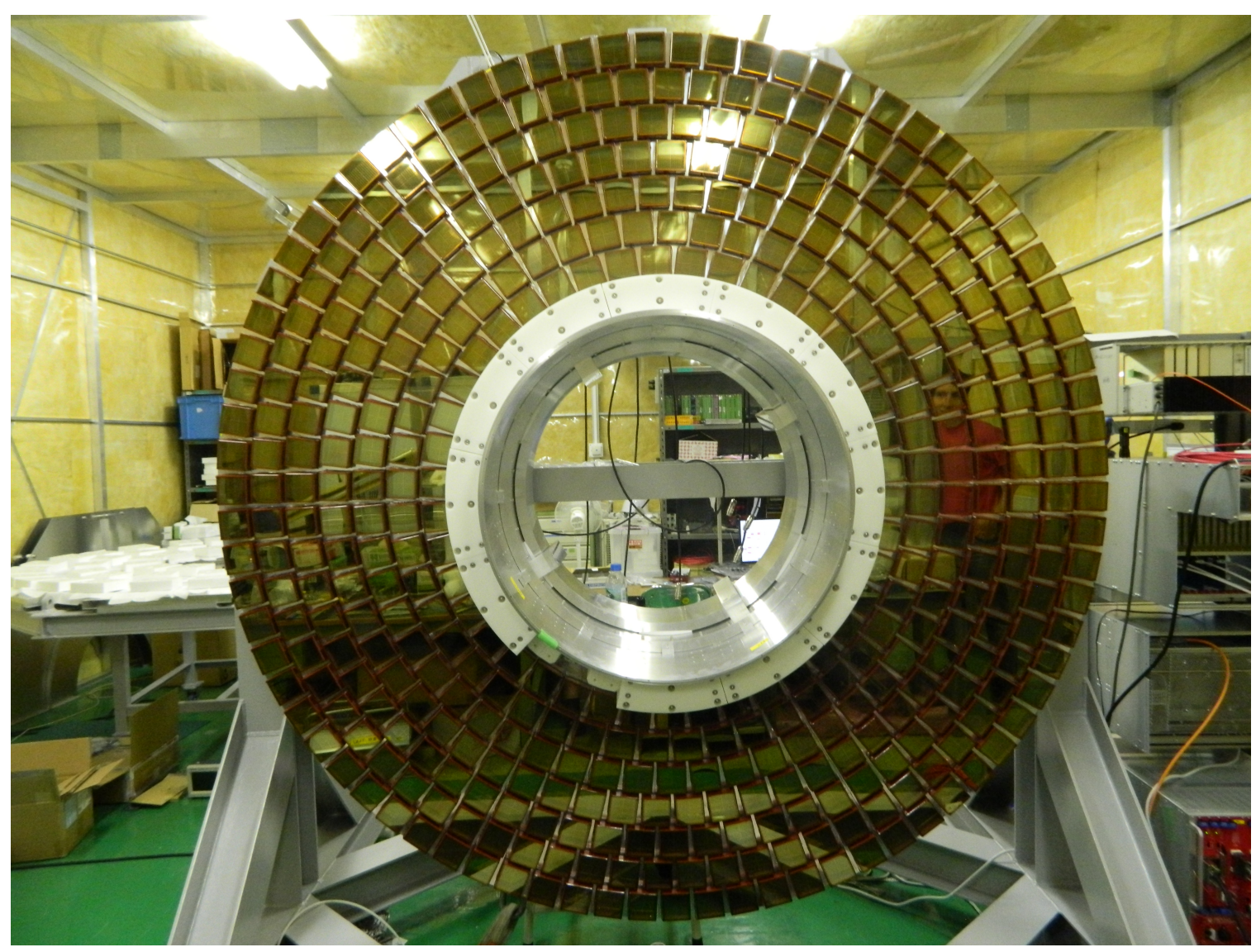

Figure 5.5: Photon detector plane with HAPD sensors of the ARICH (Courtesy: Reference [50]).

jointly with Hamamatsu $[58,59]$. HAPD sensors have dimensions of $73 \times 73 \mathrm{~mm}^{2}$ and consists of 144 channels. The photo-electrons are accelerated over a potential difference of $8 \mathrm{kV}$, and are detected in avalanche photodiodes.

\subsubsection{Electromagnetic Calorimeter (ECL)}

The electromagnetic calorimeter (ECL) [60] is a highly-segmented array of thalliumdoped caesium iodide $\mathrm{CsI}(\mathrm{Tl})$ crystals assembled in a projective geometry, as shown in Figure 5.1. All three detector regions, barrel as well as the forward and backward end-caps, are instrumented with a total of 8736 crystals, covering about $90 \%$ of the solid angle in the centre-of-mass system. Performance similar to the the Belle experiment is expected, where the energy resolution observed with the same calorimeter 
was $\sigma_{E} / E=4 \%$ at $100 \mathrm{MeV}, 1.6 \%$ at $8 \mathrm{GeV}$, and the angular resolution was $13 \mathrm{mrad}$ $(3 \mathrm{mrad})$ at low (high) energies; $\pi^{0}$ mass resolution was $4.5 \mathrm{MeV} / c^{2}$ [61].

\subsection{5 $K_{\mathrm{L}}-$ Muon Detector (KLM)}

The $K_{L}^{0}$ and muon detector (KLM) at Belle was based on glass-electrode resistive plate chambers (RPC). Since large backgrounds are expected in a high luminosity environment, the KLM system at Belle II consists of RPC only in some parts of the barrel. The two innermost layers and the endcaps at Belle II consist of layers of scintillator strips with wavelength shifting fibers, read out by silicon photomultiplier (SiPMs, Geiger mode operated APDs) as light sensors [62]. Although the high neutron background will also cause damage to the SiPMs, and will thus increase the dark count rate in the light sensor, such a detector system has been shown to operate reliably by appropriately setting the discrimination threshold during irradiation tests.

\subsection{Trigger System}

A Belle II trigger extends the list of physics analysis probed in the previous generation B-factories to a wider range of new physics potentials, including the single photon trigger for dark sector searches, and the two- and three- photon triggers for axion-like particle searches.

The Belle II trigger system is composed of two levels: hardware based low level trigger (L1) and software based high level trigger (HLT). While the nominal L1 trigger has a latency of $5 \mu \mathrm{s}$, and maximum trigger output rate of $30 \mathrm{kHz}$, it is limited by the read-in rate of the data acquisition system (DAQ). The HLT suppresses the event rate to $15 \mathrm{kHz}$ firstly with the information from the CDC track finding and ECL reconstruction. A total of $6000 \mathrm{CPU}$ cores are employed to process at the nominal $30 \mathrm{kHz}$ rate. The HLT also reconstructs the event with offline reconstruction algorithms, thereby allowing access to full granularity event reconstruction using all 
detectors except for the PXD. Only the events passing this first step are considered for full event reconstruction. Finally, the recorded event rate is further reduced to $10 \mathrm{kHz}$ by using full reconstruction information. 


\section{CHAPTER VI}

\section{DATA QUALITY MONITORING OF THE MUON DETECTOR}

\subsection{Introduction}

KLM is the largest and the outermost subdetector of Belle II [63]. Its purpose is to detect muons $(\mu)$ and long-lived neutral kaons $K_{L}^{0}$. It is an updated version of the Belle KLM detector [64, 65].

\subsection{Structure of the KLM Detector}

The KLM consists of large-area thin planar detectors interleaved with passive material consisting of iron plates which are $4.7 \mathrm{~cm}$ thick, All the active detector elements are located outside the superconducting solenoid. The iron plates serve as the magnetic flux return up to $1.5 \mathrm{~T}$ for the solenoid, providing 3.9 interaction lengths of material, beyond the 0.8 interaction lengths of the calorimeter. In the Belle experiment, gaps in the segmented flux return of the superconducting solenoidal magnet system, providing a central magnetic flux of $1.5 \mathrm{~T}$, were populated with RPCs. The main update of Belle II KLM over Belle KLM is the endcaps and two inner layers of the barrel resistive plate chambers (RPCs) were replaced with scintillatorbased detectors with silicon photomultiplier readout via wavelength shifting fiber light 


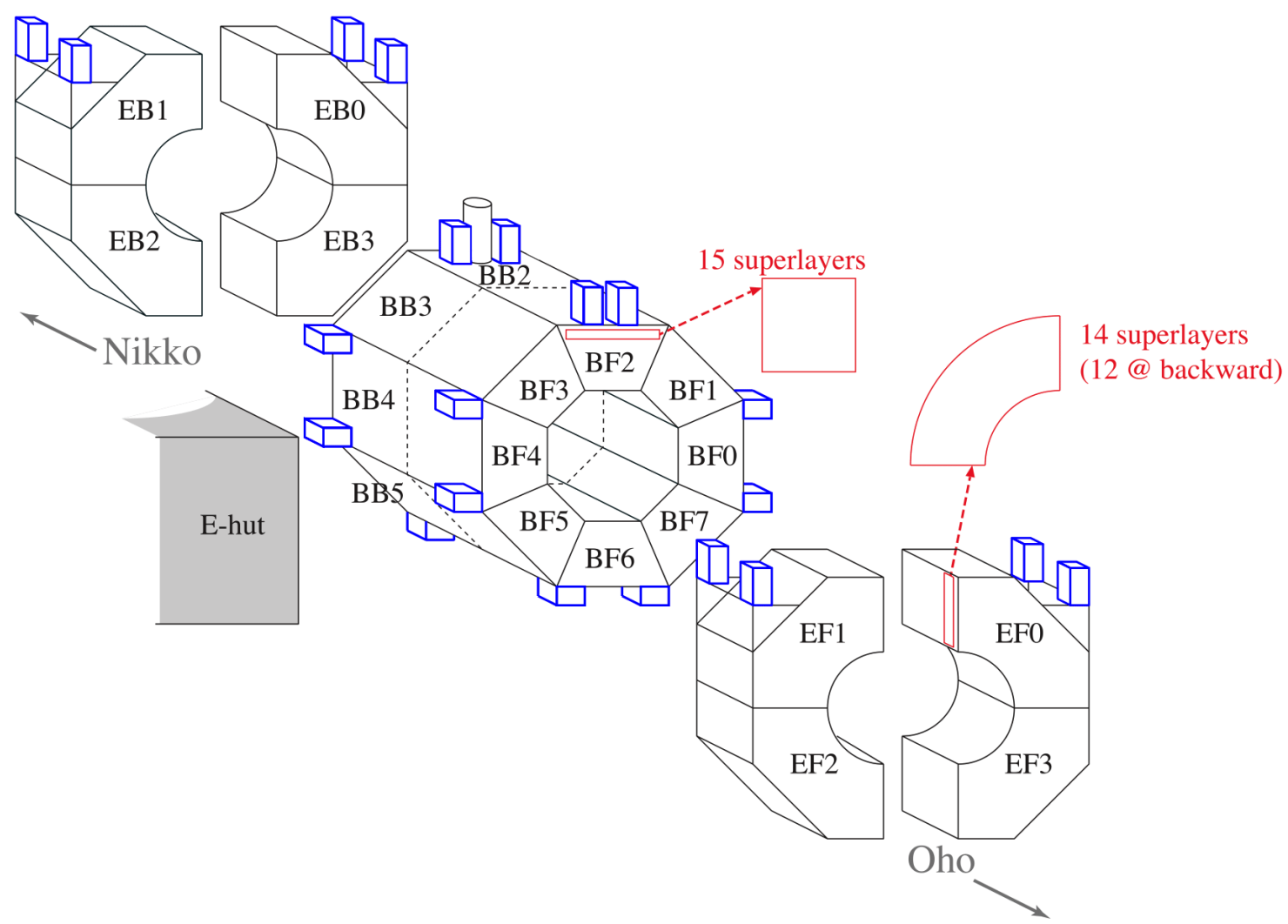

Figure 6.1: Schematics of the barrel and endcap sections of the KLM detector (Courtesy: Reference [66]).

collection. This is because of the relatively large deadtime of the RPCs and high levels of anticipated backgrounds at the Belle II experiment.

KLM is divided into a barrel (BKLM) and endcaps (EKLM), as shown in Figure 6.1. The barrel is divided into forward and backward halves, eight sectors (octants) in each half, 15 layers in each sector. Layers 0 and 1 are composed of scintillator strips, while layers 2-14 are RPCs. Endcaps are divided into four sectors (quadrants) each, 14 (12) layers in the forward (backward) endcap.

\begin{tabular}{ccc}
\hline \hline Range of $\theta$ (degrees) & Range of $z(\mathrm{~cm})$ & BKLM or EKLM \\
\hline $37<\theta<130$ & $-180<z<275$ & BKLM \\
$18<\theta<47$ or $122<\theta<155$ & $z<-180$ or $z>275$ & EKLM \\
\hline
\end{tabular}

Table 6.1: $\theta$ and $z$ discrimination for the BKLM and EKLM 


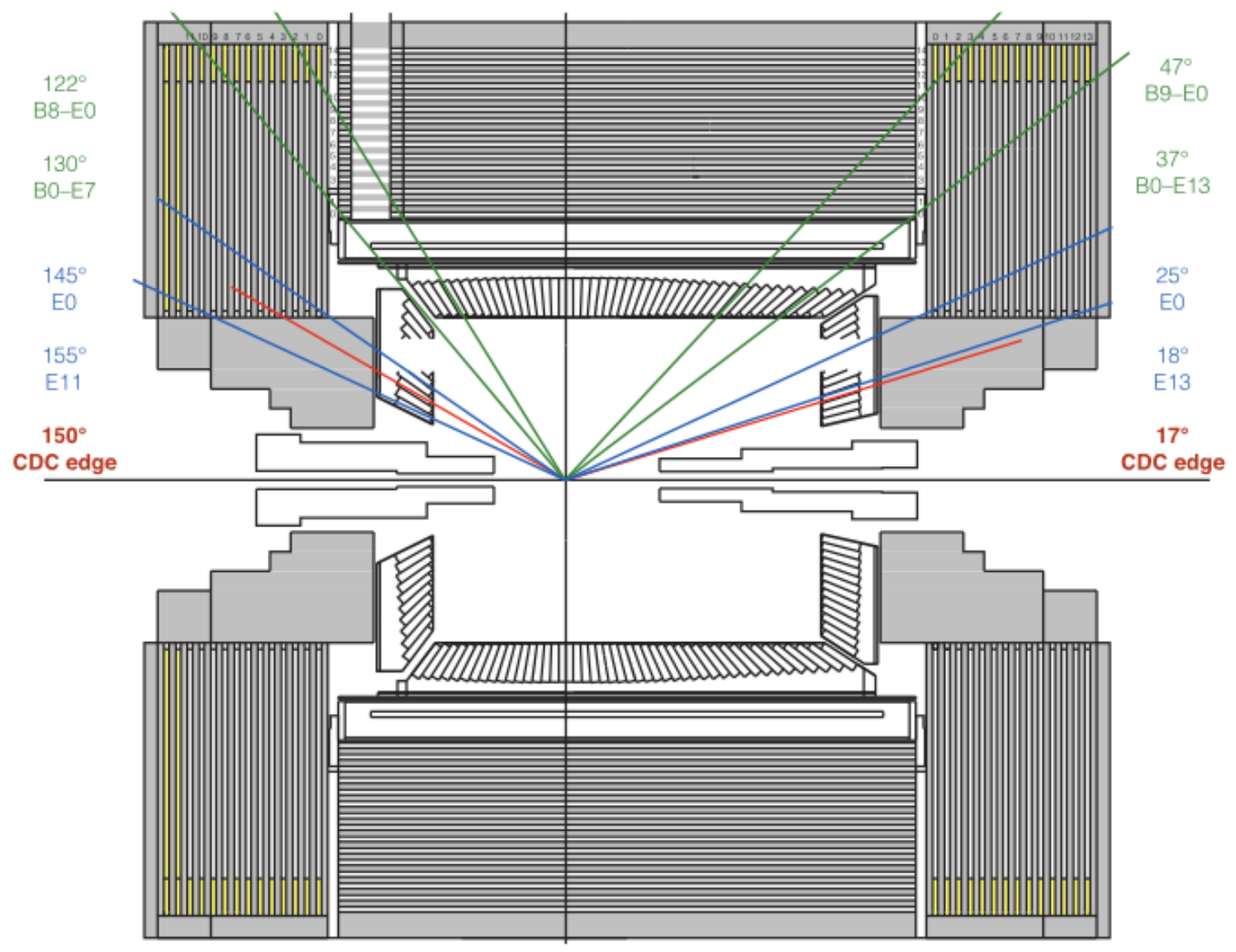

Figure 6.2: Side view of the KLM detector (Courtesy: Reference [50]). 
The KLM side view is shown in the Figure 6.2. The range of theta $(\theta)$ and $z$ are shown in Table 6.1 for BKLM and EKLM. There are $166 \mathrm{U}$ crates with RPC front-end electronics (FEE), located all around the detector. The scintillator readout electronics for the barrel are located in four $9 \mathrm{U}$ crates on top of the detector. The scintillator readout electronics for the endcap are located in eight $9 \mathrm{U}$ crates which are mounted in short racks on top of the endcap doors.

The readout of the KLM detector starts with analog signals being digitized in the FEE boards. The digitized data from ASIC-based readouts from the scintillators and discriminator-based readouts from the resistive plate chambers (RPC) are merged into Data Concentrators (DC). In the barrel (endcaps) all FEE boards of the same sector are connected to one (two) Data Concentrators (DC). The data collection from all sub-detectors at Belle II are handled by a Common Pipelined Platform for Electronics Readout (COPPER), which is a versatile data acquisition (DAQ) board equipped with various I/O cards and CPUs. Each DC is connected to (i) a High-Speed Serial Link Boards (HSLB) on a COPPER via Belle2Links, (ii) a front-end timing switch board (FTSW) for trigger-timing distribution (TTD) and (iii) a universal trigger board version 3 (UT3) which generates a KLM trigger for the Global Decision Logic (GDL) where the final online trigger decision is performed.

\subsection{KLM Data Quality}

In order to scrutinize the performance of the KLM detector in various parts, we need to create data quality monitoring (DQM) plots for KLM detector. Some of the KLM data quality plots are shown in this section.

The Figure 6.3 shows the KLM has altogther eight nodes; 4 for the BKLM and 4 for the EKLM. It also shows the 1D and 2D hits for BKLM and EKLM. The raw data are converted to bklmDigits using sector, layer, channel, time and charge informations. Finally, the 7 BKLM Digits are coverted to 1 BKLM 1D hits and 2 BKLM 

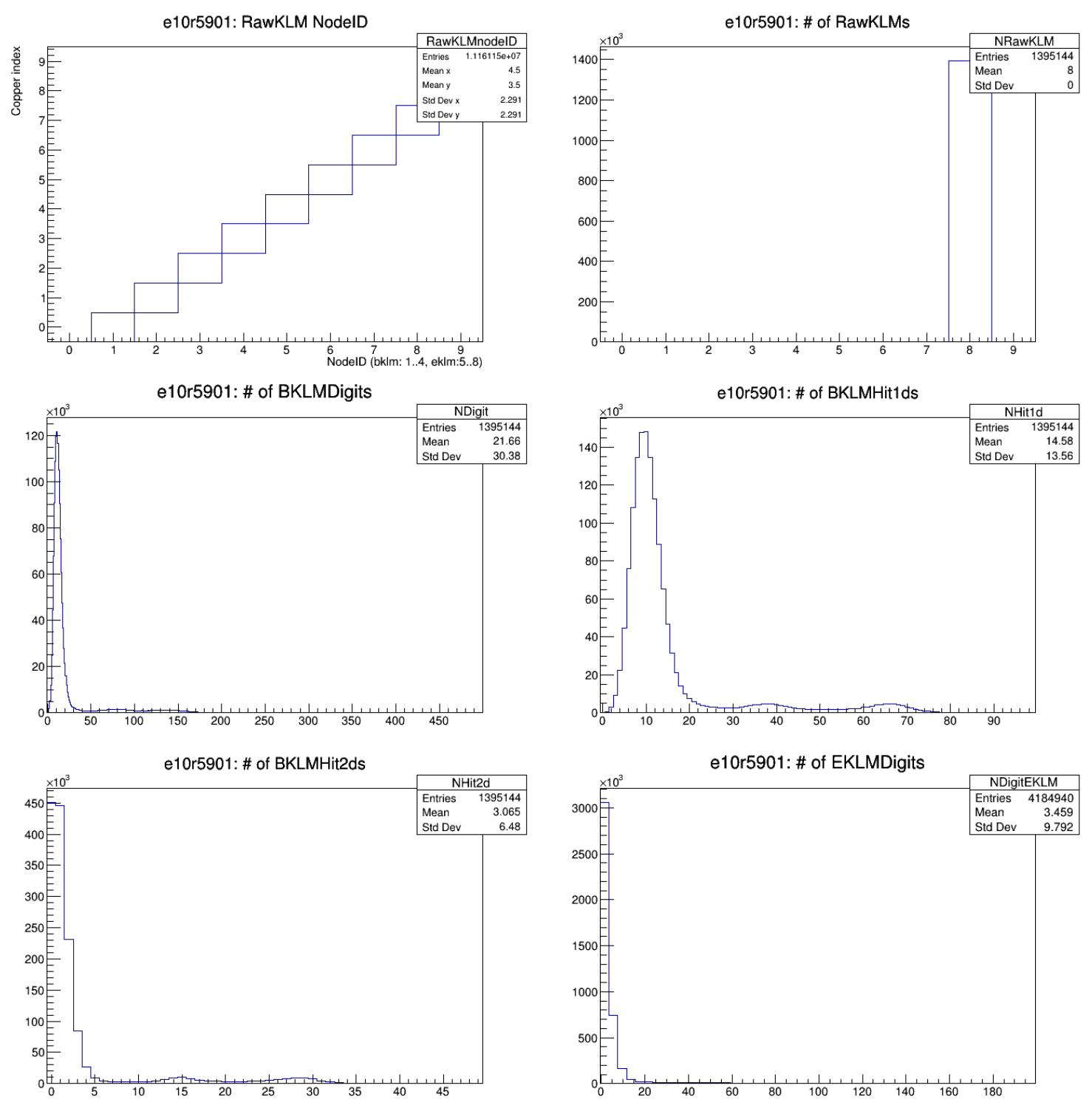

Figure 6.3: RawKLMs for Barrel and Endcap

1D Hits are converted to 1 BKLM 2D Hits.

The sector occupancy for BKLM and EKLM are shown in Figure 6.4. The $x$ vs $y$ views of hit occupancy for the BKLM, EKLM backward and EKLM forward are shown in Figures 6.5, 6.6 and 6.7, respectively. The Figures 6.3 to 6.7 correspond to experiment 10 with run number 5901. 

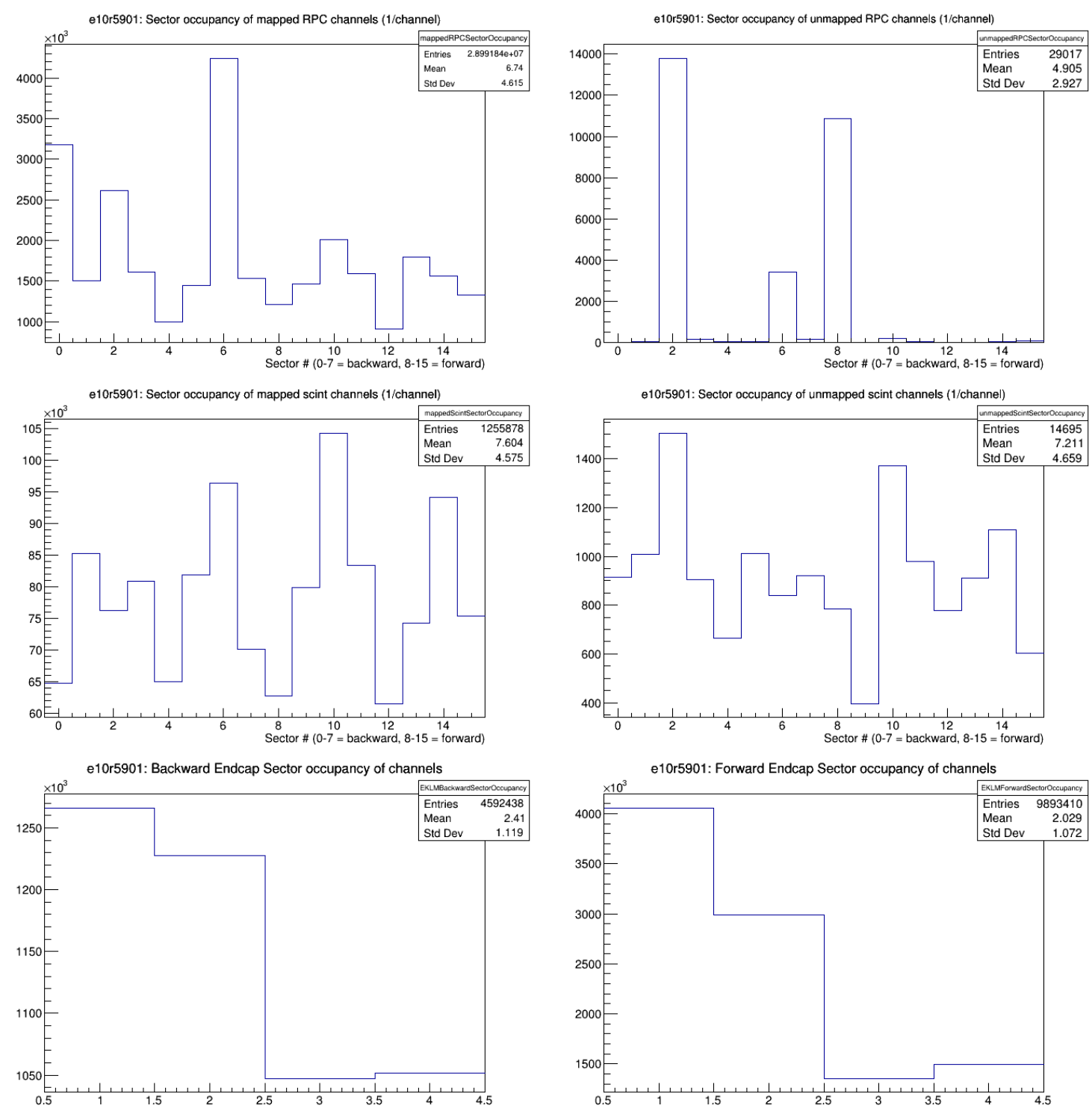

Figure 6.4: Sector occupancy for Barrel and Endcap

\subsection{Expert-level DQM webpages for KLM}

The expert-level DQM webpage has been created for the following reasons:

- to scrutinize the performance of the detector in various parts without logging in to KEKCC. 

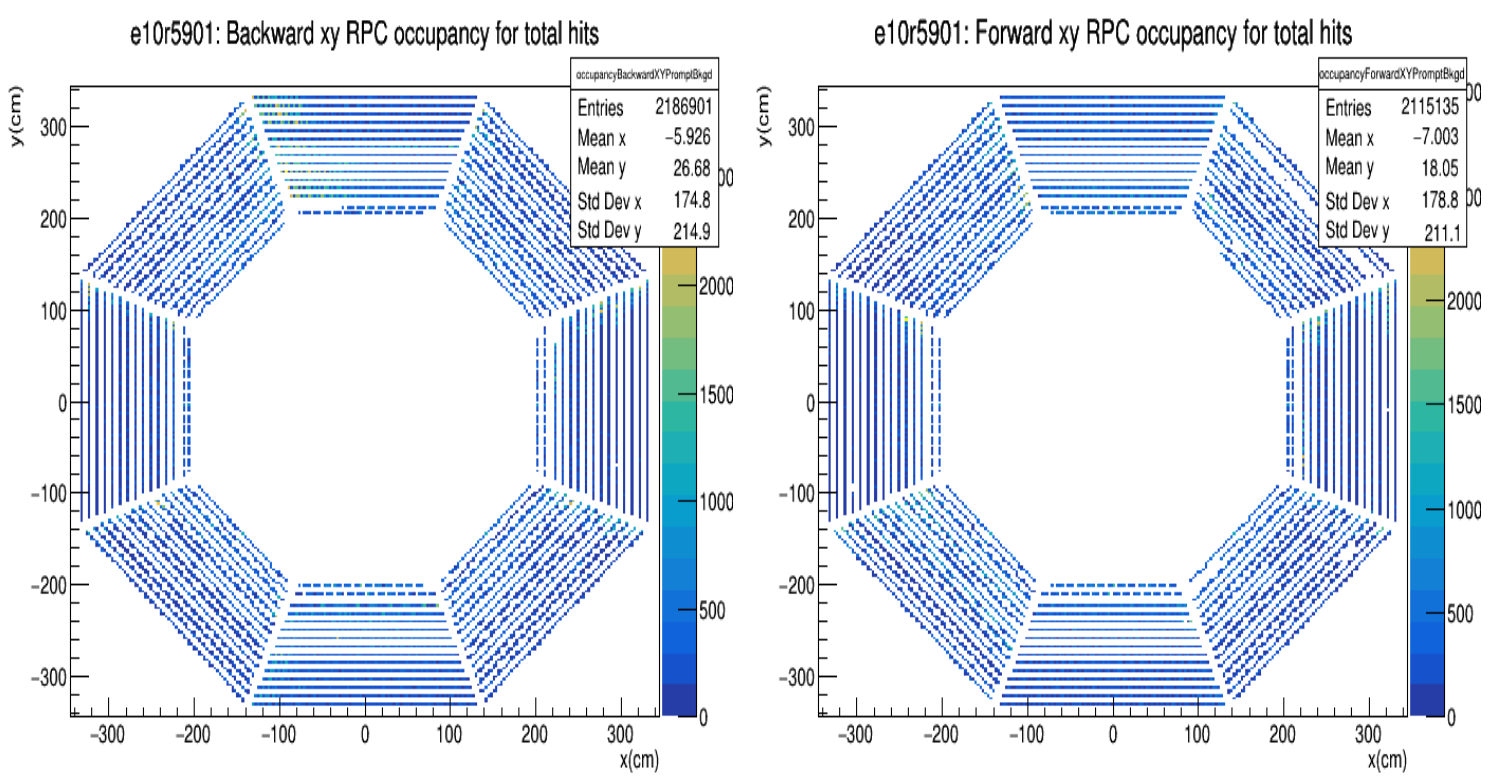

Figure 6.5: Hit occupancy for Barrel in $x$ vs $y$ view

- to generate the DQM plots dynamically, so that one can change the setting of the plots just clicking some options from inside the web-interface.

- to enable access via any computer/laptop or a mobile device for ease of portability.

I have prepared an offline library (https://dqm.belle2.org $/ \mathrm{klm} /$ ) of expertlevel DQM of the performance of both the barrel and endcap parts of the KLM detector for all runs taken during 2019 and 2020 so far. The overhead size is quite small, being of the order of 2 MBytes. Thus, a whole year of runs with more than $1 \mathrm{M}$ events can be displayed before archiving the page.

Six sequential example snapshots from a mobile device of the web-interface are shown in Figure 6.8. Such plots are crucial to diagnose the changes in data-taking conditions and check the performance of the KLM detector due to changes in threshold settings, readout calibration and trigger settings. The DQM plots have been categorized in different areas to help experts look into the specific areas if any development is needed and also prepare summary pdfs for each run. I have been regularly updating 

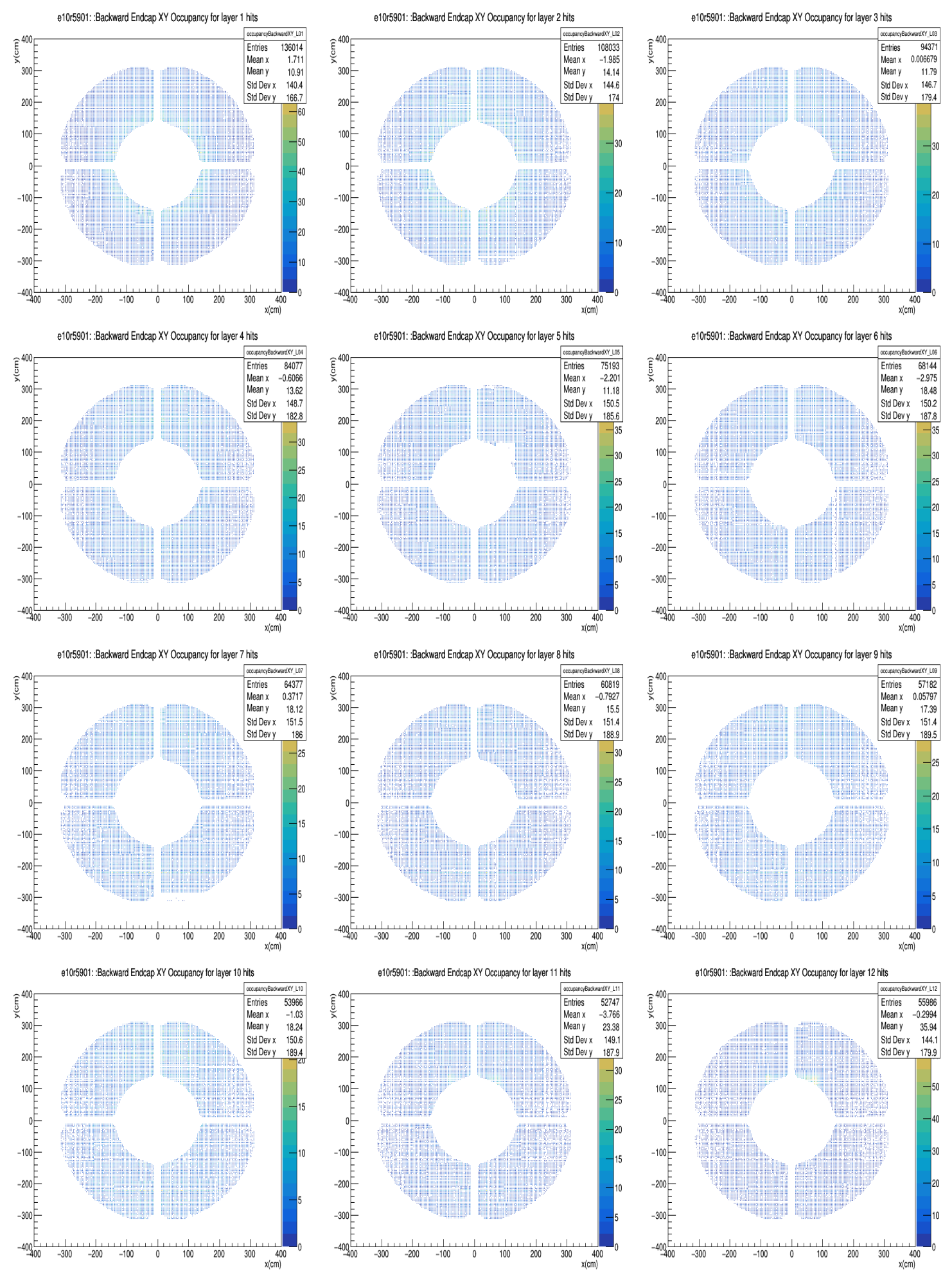

Figure 6.6: Hit occupancy for Endcap backward in $x$ vs $y$ view 

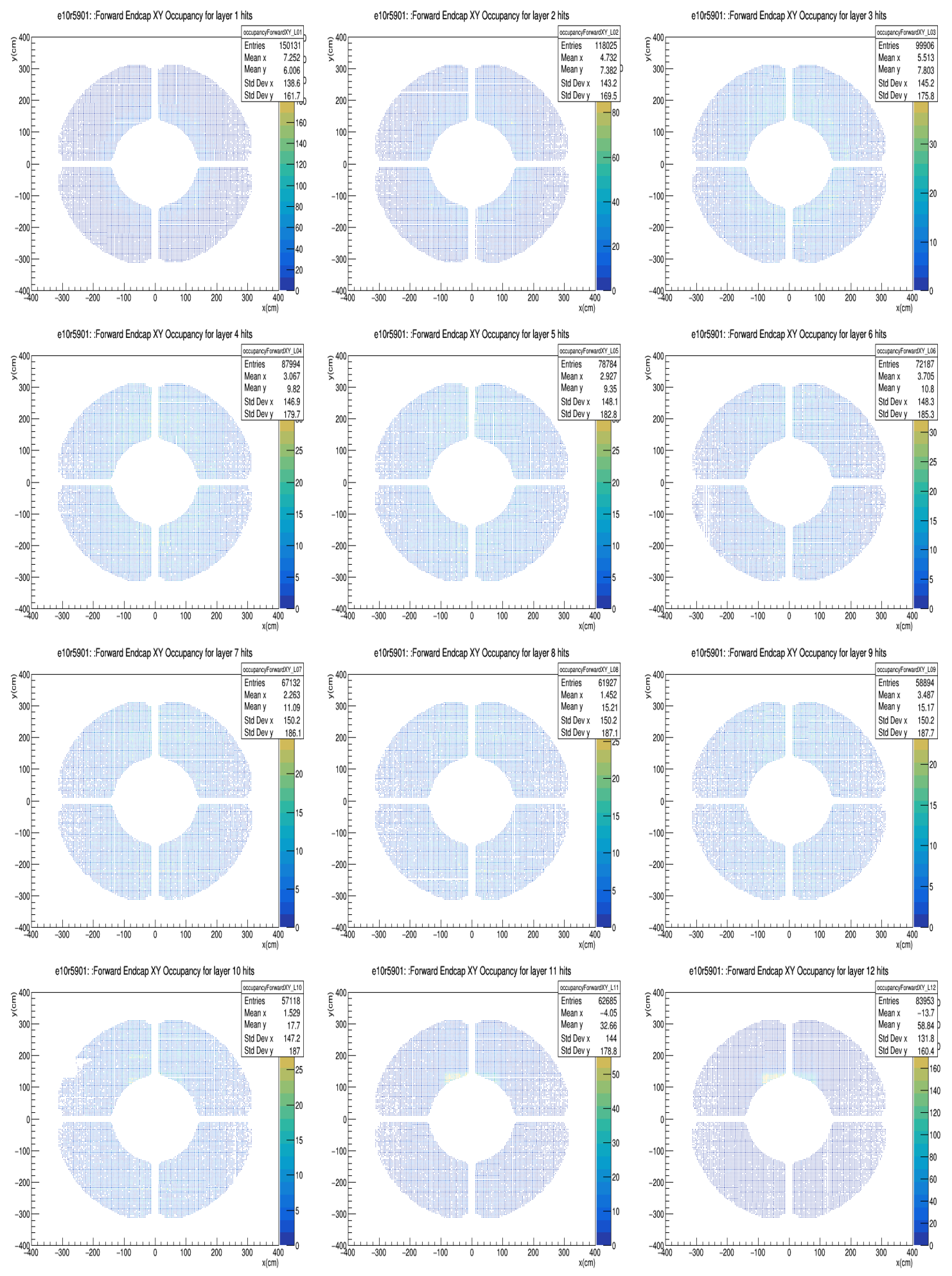

Figure 6.7: Hit occupancy for Endcap forward in $x$ vs $y$ view 
the reference set of histograms to act as a baseline for comparison with newer runs in the same data-taking period. Recently, I also updated the offline DQM webpage with the diagnostic plots from recoverable unmarked runs during 2020 data-taking period as a posterior check of KLM operations. 

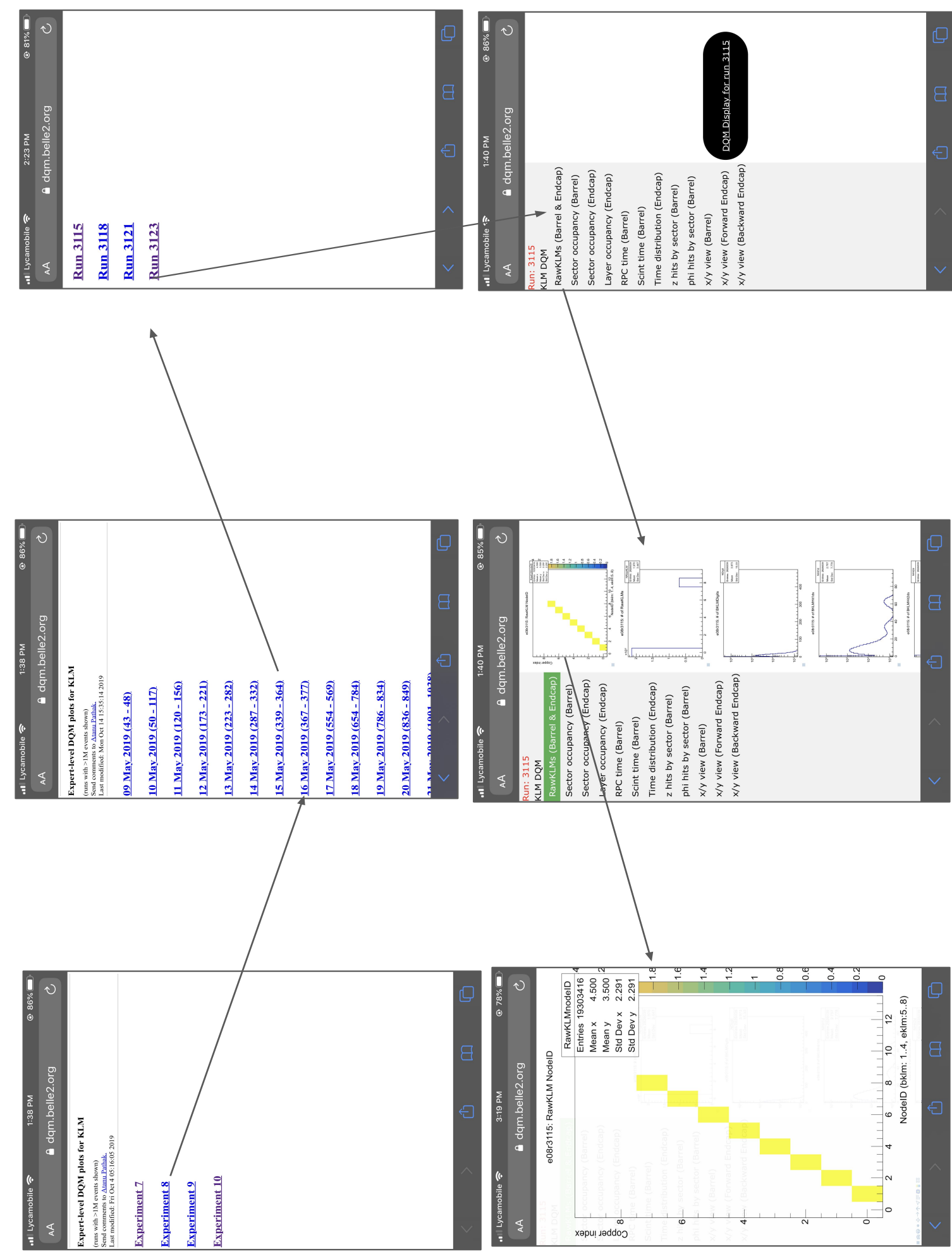

Figure 6.8: Expert-level DQM webpages for KLM. 


\section{CHAPTER VII}

\section{MODELLING OF $\tau$ DECAYS AT THE BELLE II EXPERIMENT}

\section{$7.1 \tau$ decays}

The $\tau$ lepton is an elementary particle. It is the heavier cousin of the electron with the same negative charge and spin of $\frac{1}{2}$. The decays of the $\tau$ lepton occurs through the weak interaction. The $\tau$ can decay into leptons $\left(\tau^{-} \rightarrow \ell^{-} \nu_{\tau} \bar{\nu}_{\ell}\right)$ as well as hadrons $\left(\tau^{-} \rightarrow h^{-} \nu_{\tau}\right)^{1}$ being the only lepton that can decay into hadrons. Taking into account the color charge, the naive expectation for branching fraction of $\tau$ into the electron or the muon decay channel is $20 \%$, while that of the hadronic decay mode accompanied predominantely by charged and neutral pions, and sometimes by kaons, is 60\%. Taking into account QED and QCD corrections, these branching ratios are somewhat modified, as listed in Table 7.1. These are the latest results from the PDG2020 version of a global fit to all available $\tau$ branching fraction measurements, subject to the unitarity constraint, performed by the Particle Data Group [67]. In some cases, the coefficient for each decay as in the last column of the Table 7.1 is not equal to unity, necessary to avoid double counting of similar final states appearing in decay modes containing or excluding hadronic resonances. Modes containing two

\footnotetext{
${ }^{1}$ Charge conjugate modes are implied throughout.
} 
long-lived neutral kaons $K_{L}^{0}$ are assumed to contribute as much as modes containing two short-lived neutral kaons $K_{S}^{0}$, as required by isospin symmetry. The sum of strange decay modes containing a kaon is about 3\%, while the remaining part of the hadronic decays proceed through non-strange decays. The $\tau$ decay can also be classified as 1-prong and 3-prong decays depending on the number of tracks from charged decay products. The 1-prong decays sum up to about $85 \%$, while the 3 prong decays are about $15 \%$ of all the $\tau$ decays. A small fraction of decays contains 5 charged tracks. Seven or higher prong $\tau$ decays have not been observed yet.

\begin{tabular}{lcc}
\hline \hline Decay mode & fit result (\%) & coefficient \\
\hline$\mu^{-} \bar{\nu}_{\mu} \nu_{\tau}$ & $17.3937 \pm 0.0384$ & 1.0000 \\
$e^{-} \bar{\nu}_{e} \nu_{\tau}$ & $17.8175 \pm 0.0399$ & 1.0000 \\
$\pi^{-} \nu_{\tau}$ & $10.8164 \pm 0.0512$ & 1.0000 \\
$K^{-} \nu_{\tau}$ & $0.6964 \pm 0.0096$ & 1.0000 \\
$\pi^{-} \pi^{0} \nu_{\tau}$ & $25.4941 \pm 0.0893$ & 1.0000 \\
$K^{-} \pi^{0} \nu_{\tau}$ & $0.4328 \pm 0.0148$ & 1.0000 \\
$\pi^{-} 2 \pi^{0} \nu_{\tau}\left(e x . K^{0}\right)$ & $9.2595 \pm 0.0964$ & 1.0021 \\
$K^{-} 2 \pi^{0} \nu_{\tau}\left(e x . K^{0}\right)$ & $0.0647 \pm 0.0218$ & 1.0000 \\
$\pi^{-} 3 \pi^{0} \nu_{\tau}\left(e x . K^{0}\right)$ & $1.0429 \pm 0.0707$ & 1.0000 \\
$K^{-} 3 \pi^{0} \nu_{\tau}\left(e x . K^{0}, \eta\right)$ & $0.0478 \pm 0.0212$ & 1.0000 \\
$h^{-} 4 \pi^{0} \nu_{\tau}\left(e x . K^{0}, \eta\right)$ & $0.1118 \pm 0.0391$ & 1.0000 \\
$\pi^{-} \bar{K}^{0} \nu_{\tau}$ & $0.8384 \pm 0.0138$ & 1.0000 \\
$K^{-} K^{0} \nu_{\tau}$ & $0.1486 \pm 0.0034$ & 1.0000 \\
$\pi^{-} \bar{K}^{0} \pi^{0} \nu_{\tau}$ & $0.3817 \pm 0.0129$ & 1.0000 \\
$K^{-} \pi^{0} K^{0} \nu_{\tau}$ & $0.1500 \pm 0.0070$ & 1.0000 \\
$\pi^{-} \bar{K}^{0} 2 \pi^{0} \nu_{\tau}\left(e x . K^{0}\right)$ & $0.0263 \pm 0.0226$ & 1.0000 \\
$\pi^{-} K_{S}^{0} K_{S}^{0} \nu_{\tau}$ & $0.0235 \pm 0.0006$ & 2.0000 \\
$\pi^{-} K_{S}^{0} K_{L}^{0} \nu_{\tau}$ & $0.1081 \pm 0.0241$ & 1.0000 \\
\hline \hline
\end{tabular}




\begin{tabular}{|c|c|c|}
\hline Decay mode & fit result $(\%)$ & coefficient \\
\hline$\pi^{-} \pi^{0} K_{S}^{0} K_{S}^{0} \nu_{\tau}$ & $0.0018 \pm 0.0002$ & 2.0000 \\
\hline$\pi^{-} \pi^{0} K_{S}^{0} K_{L}^{0} \nu_{\tau}$ & $0.0325 \pm 0.0119$ & 1.0000 \\
\hline $\bar{K}^{0} h^{-} h^{-} h^{+} \nu_{\tau}$ & $0.0247 \pm 0.0199$ & 1.0000 \\
\hline$\pi^{-} \pi^{-} \pi^{+} \nu_{\tau}\left(e x . K^{0}, \omega\right)$ & $8.9868 \pm 0.0513$ & 1.0021 \\
\hline$\pi^{-} \pi^{-} \pi^{+} \pi^{0} \nu_{\tau}\left(e x . K^{0}, \omega\right)$ & $2.7404 \pm 0.0710$ & 1.0000 \\
\hline$h^{-} h^{-} h^{+} 2 \pi^{0} \nu_{\tau}\left(e x . K^{0}, \omega, \eta\right)$ & $0.0981 \pm 0.0356$ & 1.0000 \\
\hline$\pi^{-} K^{-} K^{+} \nu_{\tau}$ & $0.1435 \pm 0.0027$ & 1.0000 \\
\hline$\pi^{-} K^{-} K^{+} \pi^{0} \nu_{\tau}$ & $0.0061 \pm 0.0018$ & 1.0000 \\
\hline$\pi^{-} \pi^{0} \eta \nu_{\tau}$ & $0.1389 \pm 0.0072$ & 1.0000 \\
\hline$K^{-} \eta \nu_{\tau}$ & $0.0155 \pm 0.0008$ & 1.0000 \\
\hline$K^{-} \pi^{0} \eta \nu_{\tau}$ & $0.0048 \pm 0.0012$ & 1.0000 \\
\hline$\pi^{-} \bar{K}^{0} \eta \nu_{\tau}$ & $0.0094 \pm 0.0015$ & 1.0000 \\
\hline$\pi^{-} \pi^{+} \pi^{-} \eta \nu_{\tau}\left(e x . K^{0}\right)$ & $0.0220 \pm 0.0013$ & 1.0000 \\
\hline$K^{-} \omega \nu_{\tau}$ & $0.0410 \pm 0.0092$ & 1.0000 \\
\hline$h^{-} \pi^{0} \omega \nu_{\tau}$ & $0.4085 \pm 0.0419$ & 1.0000 \\
\hline$K^{-} \phi \nu_{\tau}$ & $0.0044 \pm 0.0016$ & 0.8320 \\
\hline$\pi^{-} \omega \nu_{\tau}$ & $1.9494 \pm 0.0645$ & 1.0000 \\
\hline$K^{-} \pi^{-} \pi^{+} \nu_{\tau}\left(e x . K^{0}, \omega\right)$ & $0.2927 \pm 0.0068$ & 1.0000 \\
\hline$K^{-} \pi^{-} \pi^{+} \pi^{0} \nu_{\tau}\left(e x . K^{0}, \omega, \eta\right)$ & $0.0394 \pm 0.0142$ & 1.0000 \\
\hline$\pi^{-} 2 \pi^{0} \omega \nu_{\tau}\left(e x . K^{0}\right)$ & $0.0072 \pm 0.0016$ & 1.0000 \\
\hline $2 \pi^{-} \pi^{+} 3 \pi^{0} \nu_{\tau}\left(e x . K^{0}, \eta, \omega, f_{1}\right)$ & $0.0014 \pm 0.0027$ & 1.0000 \\
\hline $3 \pi^{-} 2 \pi^{+} \nu_{\tau}\left(e x . K^{0}, \omega, f_{1}\right)$ & $0.0775 \pm 0.0030$ & 1.0000 \\
\hline$K^{-} 2 \pi^{-} 2 \pi^{+} \nu_{\tau}\left(e x . K^{0}\right)$ & $0.0001 \pm 0.0001$ & 1.0000 \\
\hline $2 \pi^{-} \pi^{+} \omega \nu_{\tau}\left(e x . K^{0}\right)$ & $0.0084 \pm 0.0006$ & 1.0000 \\
\hline $3 \pi^{-} 2 \pi^{+} \pi^{0} \nu_{\tau}\left(e x . K^{0}, \eta, \omega, f_{1}\right)$ & $0.0038 \pm 0.0009$ & 1.0000 \\
\hline$K^{-} 2 \pi^{-} 2 \pi^{+} \pi^{0} \nu_{\tau}\left(e x . K^{0}\right)$ & $0.0001 \pm 0.0001$ & 1.0000 \\
\hline
\end{tabular}




\begin{tabular}{lcc}
\hline \hline Decay mode & fit result (\%) & coefficient \\
\hline$\pi^{-} f_{1} \nu_{\tau}\left(f_{1} \rightarrow 2 \pi^{-} 2 \pi^{+}\right)$ & $0.0052 \pm 0.0004$ & 1.0000 \\
$\pi^{-} 2 \pi^{0} \eta \nu_{\tau}$ & $0.0195 \pm 0.0038$ & 1.0000 \\
\hline \hline
\end{tabular}

Table 7.1: $\tau$ branching fractions from PDG2020 version [67].

\subsection{TAUOLA-BBB Monte Carlo}

In the current version of Belle II software (release-04-02-08), $e^{-} e^{+} \rightarrow \tau^{-} \tau^{+}$ events are generated using KKMC [68] and subsequent $\tau$ decays are handled by TAUOLA [69] and PYTHIA [70]. Here update of the TAUOLA Monte Carlo to the TAUOLA-BBB version [71] in the Belle II software is presented [72]. This version allows users to choose between different parameterizations of the underlying current and pre-sample the available phase space with higher precision necessary avoid soft and collinear divergences in the calculation of invariant mass of $e^{-} e^{+}$pairs [73]. Belle II initialization of the TAUOLA-BBB version to the PDG2020 version of $\tau$ branching fractions [67] for 92 $\tau^{-}$decay modes and their branching fractions $(\mathrm{BF})$ are listed in Table 7.2.

\begin{tabular}{lcc}
\hline \hline Decay mode & TauBBMode & BF \\
\hline$\tau^{-} \rightarrow e^{-} \bar{\nu}_{e} \nu_{\tau}$ & 1 & $0.178175 \pm 0.000399$ \\
\hline$\tau^{-} \rightarrow e^{-} e^{-} e^{+} \bar{\nu}_{e} \nu_{\tau}$ & 5 & $0.000028 \pm 0.000015$ \\
\hline$\tau^{-} \rightarrow \mu^{-} \bar{\nu}_{\mu} \nu_{\tau}$ & 2 & $0.173937 \pm 0.000384$ \\
\hline$\tau^{-} \rightarrow \pi^{-} \nu_{\tau}$ & 303 & $0.108164 \pm 0.000512$ \\
\hline$\tau^{-} \rightarrow \rho^{-}\left(\rightarrow \pi^{-} \gamma\right) \nu_{\tau}$ & 342 & $0.000115 \pm 0.000001$ \\
\hline$\tau^{-} \rightarrow a_{1}^{-}\left(\rightarrow \pi^{-} \gamma\right) \nu_{\tau}$ & 341 & $0.000238 \pm 0.0000002$ \\
\hline$\tau^{-} \rightarrow K^{-} \nu_{\tau}$ & 304 & $0.006964 \pm 0.0000096$ \\
\hline$\tau^{-} \rightarrow \pi^{-} \pi^{0} \nu_{\tau}$ & 163 & $0.254941 \pm 0.000893$ \\
\hline$\tau^{-} \rightarrow \pi^{-} \omega\left(\rightarrow \pi^{0} \gamma\right) \nu_{\tau}$ & 110 & $0.001789 \pm 0.005914$ \\
\hline \hline
\end{tabular}




\begin{tabular}{|c|c|c|}
\hline Decay mode & TauBBMode & $\mathrm{BF}$ \\
\hline$\tau^{-} \rightarrow K^{-} \pi^{0} \nu_{\tau}$ & 165 & $0.004328 \pm 0.000148$ \\
\hline$\tau^{-} \rightarrow \pi^{-} 2 \pi^{0} \nu_{\tau}$ & 111 & $0.092595 \pm 0.000964$ \\
\hline$\tau^{-} \rightarrow \pi^{-} \pi^{0} \omega\left(\rightarrow \pi^{0} \gamma\right) \nu_{\tau}$ & 132 & $0.000374 \pm 0.000038$ \\
\hline$\tau^{-} \rightarrow K^{-} 2 \pi^{0} \nu_{\tau}$ & 106 & $0.000647 \pm 0.000218$ \\
\hline$\tau^{-} \rightarrow \pi^{-} 3 \pi^{0} \nu_{\tau}$ & 4 & $0.010429 \pm 0.000707$ \\
\hline$\tau^{-} \rightarrow K^{-} 3 \pi^{0} \nu_{\tau}$ & 9 & $0.000478 \pm 0.000212$ \\
\hline$\tau^{-} \rightarrow \pi^{-} 4 \pi^{0} \nu_{\tau}$ & 67 & $0.001118 \pm 0.000391$ \\
\hline$\tau^{-} \rightarrow \pi^{-} K_{S}^{0} \nu_{\tau}$ & 226 & $0.004192 \pm 0.000069$ \\
\hline$\tau^{-} \rightarrow \pi^{-} K_{L}^{0} \nu_{\tau}$ & 227 & $0.004192 \pm 0.000069$ \\
\hline$\tau^{-} \rightarrow K^{-} K_{S}^{0} \nu_{\tau}$ & 228 & $0.000743 \pm 0.000017$ \\
\hline$\tau^{-} \rightarrow K^{-} K_{L}^{0} \nu_{\tau}$ & 229 & $0.000743 \pm 0.000017$ \\
\hline$\tau^{-} \rightarrow \pi^{-} K_{S}^{0} \pi^{0} \nu_{\tau}$ & 126 & $0.001909 \pm 0.000065$ \\
\hline$\tau^{-} \rightarrow \pi^{-} K_{L}^{0} \pi^{0} \nu_{\tau}$ & 127 & $0.001909 \pm 0.000065$ \\
\hline$\tau^{-} \rightarrow K^{-} \pi^{0} K_{S}^{0} \nu_{\tau}$ & 124 & $0.000750 \pm 0.000035$ \\
\hline$\tau^{-} \rightarrow K^{-} \pi^{0} K_{L}^{0} \nu_{\tau}$ & 125 & $0.000750 \pm 0.000035$ \\
\hline$\tau^{-} \rightarrow \pi^{-} 2 \pi^{0} K_{S}^{0} \nu_{\tau}$ & 30 & $0.000131 \pm 0.000113$ \\
\hline$\tau^{-} \rightarrow \pi^{-} 2 \pi^{0} K_{L}^{0} \nu_{\tau}$ & 31 & $0.000131 \pm 0.000113$ \\
\hline$\tau^{-} \rightarrow \pi^{-} K_{S}^{0} K_{S}^{0} \nu_{\tau}$ & 121 & $0.000235 \pm 0.000006$ \\
\hline$\tau^{-} \rightarrow \pi^{-} K_{L}^{0} K_{L}^{0} \nu_{\tau}$ & 122 & $0.000235 \pm 0.000006$ \\
\hline$\tau^{-} \rightarrow \pi^{-} K_{S}^{0} K_{L}^{0} \nu_{\tau}$ & 123 & $0.001081 \pm 0.000241$ \\
\hline$\tau^{-} \rightarrow \pi^{-} \pi^{0} K_{S}^{0} K_{S}^{0} \nu_{\tau}$ & 34 & $0.000018 \pm 0.000002$ \\
\hline$\tau^{-} \rightarrow \pi^{-} \pi^{0} K_{L}^{0} K_{L}^{0} \nu_{\tau}$ & 35 & $0.000018 \pm 0.000002$ \\
\hline$\tau^{-} \rightarrow \pi^{-} \pi^{0} K_{S}^{0} K_{L}^{0} \nu_{\tau}$ & 36 & $0.000325 \pm 0.000119$ \\
\hline$\tau^{-} \rightarrow \pi^{-} \pi^{0} \eta(\rightarrow \gamma \gamma) \nu_{\tau}$ & 133 & $0.000547 \pm 0.000028$ \\
\hline$\tau^{-} \rightarrow \pi^{-} \pi^{0} \eta\left(\rightarrow \pi^{0} \pi^{0} \pi^{0}\right) \nu_{\tau}$ & 134 & $0.000454 \pm 0.000024$ \\
\hline
\end{tabular}




\begin{tabular}{|c|c|c|}
\hline Decay mode & TauBBMode & $\mathrm{BF}$ \\
\hline$\tau^{-} \rightarrow \pi^{-} \pi^{0} \eta\left(\rightarrow \pi^{-} \pi^{+} \pi^{0}\right) \nu_{\tau}$ & 135 & $0.000318 \pm 0.000017$ \\
\hline$\tau^{-} \rightarrow \pi^{-} \pi^{0} \eta\left(\rightarrow \pi^{-} \pi^{+} \gamma\right) \nu_{\tau}$ & 136 & $0.000069 \pm 0.000004$ \\
\hline$\tau^{-} \rightarrow K^{-} \eta(\rightarrow \gamma \gamma) \nu_{\tau}$ & 242 & $0.000061 \pm 0.000003$ \\
\hline$\tau^{-} \rightarrow K^{-} \eta\left(\rightarrow \pi^{0} \pi^{0} \pi^{0}\right) \nu_{\tau}$ & 243 & $0.000050 \pm 0.000003$ \\
\hline$\tau^{-} \rightarrow K^{-} \eta\left(\rightarrow \pi^{-} \pi^{+} \pi^{0}\right) \nu_{\tau}$ & 244 & $0.000036 \pm 0.000002$ \\
\hline$\tau^{-} \rightarrow K^{-} \eta\left(\rightarrow \pi^{-} \pi^{+} \gamma\right) \nu_{\tau}$ & 245 & $0.000008 \pm 0.000001$ \\
\hline$\tau^{-} \rightarrow K^{-} \pi^{0} \eta(\rightarrow \gamma \gamma) \nu_{\tau}$ & 137 & $0.000019 \pm 0.000005$ \\
\hline$\tau^{-} \rightarrow K^{-} \pi^{0} \eta\left(\rightarrow \pi^{0} \pi^{0} \pi^{0}\right) \nu_{\tau}$ & 138 & $0.000016 \pm 0.000004$ \\
\hline$\tau^{-} \rightarrow K^{-} \pi^{0} \eta\left(\rightarrow \pi^{-} \pi^{+} \pi^{0}\right) \nu_{\tau}$ & 139 & $0.000011 \pm 0.000003$ \\
\hline$\tau^{-} \rightarrow K^{-} \pi^{0} \eta\left(\rightarrow \pi^{-} \pi^{+} \gamma\right) \nu_{\tau}$ & 140 & $0.000002 \pm 0.000001$ \\
\hline$\tau^{-} \rightarrow \pi^{-} K_{S}^{0} \eta(\rightarrow \gamma \gamma) \nu_{\tau}$ & 141 & $0.000019 \pm 0.000003$ \\
\hline$\tau^{-} \rightarrow \pi^{-} K_{S}^{0} \eta\left(\rightarrow \pi^{0} \pi^{0} \pi^{0}\right) \nu_{\tau}$ & 142 & $0.000015 \pm 0.000003$ \\
\hline$\tau^{-} \rightarrow \pi^{-} K_{S}^{0} \eta\left(\rightarrow \pi^{-} \pi^{+} \pi^{0}\right) \nu_{\tau}$ & 143 & $0.000011 \pm 0.000002$ \\
\hline$\tau^{-} \rightarrow \pi^{-} K_{S}^{0} \eta\left(\rightarrow \pi^{-} \pi^{+} \gamma\right) \nu_{\tau}$ & 144 & $0.000002 \pm 0.000001$ \\
\hline$\tau^{-} \rightarrow \pi^{-} K_{L}^{0} \eta(\rightarrow \gamma \gamma) \nu_{\tau}$ & 145 & $0.000019 \pm 0.000003$ \\
\hline$\tau^{-} \rightarrow \pi^{-} K_{L}^{0} \eta\left(\rightarrow \pi^{0} \pi^{0} \pi^{0}\right) \nu_{\tau}$ & 146 & $0.000015 \pm 0.000003$ \\
\hline$\tau^{-} \rightarrow \pi^{-} K_{L}^{0} \eta\left(\rightarrow \pi^{-} \pi^{+} \pi^{0}\right) \nu_{\tau}$ & 147 & $0.000011 \pm 0.000002$ \\
\hline$\tau^{-} \rightarrow \pi^{-} K_{L}^{0} \eta\left(\rightarrow \pi^{-} \pi^{+} \gamma\right) \nu_{\tau}$ & 148 & $0.000002 \pm 0.000001$ \\
\hline$\tau^{-} \rightarrow 2 \pi^{-} \pi^{+} \nu_{\tau}$ & 112 & $0.089868 \pm 0.000513$ \\
\hline$\tau^{-} \rightarrow \pi^{-} \omega\left(\rightarrow \pi^{-} \pi^{+}\right) \nu_{\tau}$ & 237 & $0.000298 \pm 0.000010$ \\
\hline$\tau^{-} \rightarrow \pi^{-} \omega\left(\rightarrow \pi^{-} \pi^{+} \pi^{0}\right) \nu_{\tau}$ & 3 & $0.044812 \pm 0.000936$ \\
\hline$\tau^{-} \rightarrow \pi^{-} \pi^{0} \omega\left(\rightarrow \pi^{-} \pi^{+}\right) \nu_{\tau}$ & 131 & $0.000063 \pm 0.000006$ \\
\hline$\tau^{-} \rightarrow \pi^{-} \pi^{0} \omega\left(\rightarrow \pi^{-} \pi^{+} \pi^{0}\right) \nu_{\tau}$ & 66 & $0.004629 \pm 0.000532$ \\
\hline$\tau^{-} \rightarrow \pi^{-} K^{-} K^{+} \nu_{\tau}$ & 103 & $0.001435 \pm 0.000027$ \\
\hline$\tau^{-} \rightarrow \pi^{-} K^{-} K^{+} \pi^{0} \nu_{\tau}$ & 23 & $0.000061 \pm 0.000018$ \\
\hline
\end{tabular}




\begin{tabular}{|c|c|c|}
\hline Decay mode & TauBBMode & $\mathrm{BF}$ \\
\hline$\tau^{-} \rightarrow 2 \pi^{-} \pi^{+} K_{S}^{0} \nu_{\tau}$ & 32 & $0.000123 \pm 0.000099$ \\
\hline$\tau^{-} \rightarrow 2 \pi^{-} \pi^{+} K_{L}^{0} \nu_{\tau}$ & 33 & $0.000123 \pm 0.000099$ \\
\hline$\tau^{-} \rightarrow 2 \pi^{-} \pi^{+} \eta(\rightarrow \gamma \gamma) \nu_{\tau}$ & 41 & $0.000037 \pm 0.000006$ \\
\hline$\tau^{-} \rightarrow 2 \pi^{-} \pi^{+} \eta\left(\rightarrow \pi^{0} \pi^{0} \pi^{0}\right) \nu_{\tau}$ & 42 & $0.000031 \pm 0.000005$ \\
\hline$\tau^{-} \rightarrow 2 \pi^{-} \pi^{+} \eta\left(\rightarrow \pi^{-} \pi^{+} \pi^{0}\right) \nu_{\tau}$ & 43 & $0.000022 \pm 0.000003$ \\
\hline$\tau^{-} \rightarrow 2 \pi^{-} \pi^{+} \eta\left(\rightarrow \pi^{-} \pi^{+} \gamma\right) \nu_{\tau}$ & 44 & $0.000005 \pm 0.000001$ \\
\hline$\tau^{-} \rightarrow 2 \pi^{-} \pi^{+} \omega\left(\rightarrow \pi^{-} \pi^{+} \pi^{0}\right) \nu_{\tau}$ & 45 & $0.000075 \pm 0.000005$ \\
\hline$\tau^{-} \rightarrow 2 \pi^{-} \pi^{+} \omega\left(\rightarrow \pi^{-} \pi^{+}\right) \nu_{\tau}$ & 46 & $0.000001 \pm 0.000001$ \\
\hline$\tau^{-} \rightarrow 2 \pi^{-} \pi^{+} \omega\left(\rightarrow \pi^{0} \gamma\right) \nu_{\tau}$ & 47 & $0.000008 \pm 0.000001$ \\
\hline$\tau^{-} \rightarrow K^{-} \omega\left(\rightarrow \pi^{-} \pi^{+} \pi^{0}\right) \nu_{\tau}$ & 250 & $0.000366 \pm 0.000082$ \\
\hline$\tau^{-} \rightarrow K^{-} \omega\left(\rightarrow \pi^{-} \pi^{+}\right) \nu_{\tau}$ & 251 & $0.000006 \pm 0.000001$ \\
\hline$\tau^{-} \rightarrow K^{-} \omega\left(\rightarrow \pi^{0} \gamma\right) \nu_{\tau}$ & 252 & $0.000038 \pm 0.000008$ \\
\hline$\tau^{-} \rightarrow K^{-} \phi\left(\rightarrow K^{-} K^{+}\right) \nu_{\tau}$ & 230 & $0.000022 \pm 0.000013$ \\
\hline$\tau^{-} \rightarrow K^{-} \phi\left(\rightarrow K_{S}^{0} K_{L}^{0}\right) \nu_{\tau}$ & 231 & $0.000015 \pm 0.000013$ \\
\hline$\tau^{-} \rightarrow K^{-} \pi^{-} \pi^{+} \nu_{\tau}$ & 107 & $0.002927 \pm 0.000068$ \\
\hline$\tau^{-} \rightarrow K^{-} \pi^{-} \pi^{+} \pi^{0} \nu_{\tau}$ & 13 & $0.000394 \pm 0.000142$ \\
\hline$\tau^{-} \rightarrow \pi^{-} 2 \pi^{0} \omega\left(\rightarrow \pi^{-} \pi^{+} \pi^{0}\right) \nu_{\tau}$ & 48 & $0.000064 \pm 0.000014$ \\
\hline$\tau^{-} \rightarrow \pi^{-} 2 \pi^{0} \omega\left(\rightarrow \pi^{-} \pi^{+}\right) \nu_{\tau}$ & 49 & $0.000001 \pm 0.000001$ \\
\hline$\tau^{-} \rightarrow \pi^{-} 2 \pi^{0} \omega\left(\rightarrow \pi^{0} \gamma\right) \nu_{\tau}$ & 50 & $0.000007 \pm 0.000001$ \\
\hline$\tau^{-} \rightarrow 2 \pi^{-} \pi^{+} 3 \pi^{0} \nu_{\tau}$ & 85 & $0.000014 \pm 0.000027$ \\
\hline$\tau^{-} \rightarrow 3 \pi^{-} 2 \pi^{+} \nu_{\tau}$ & 68 & $0.000775 \pm 0.000030$ \\
\hline$\tau^{-} \rightarrow K^{-} 2 \pi^{-} 2 \pi^{+} \nu_{\tau}$ & 69 & $0.000001 \pm 0.000001$ \\
\hline$\tau^{-} \rightarrow 3 \pi^{-} 2 \pi^{+} \pi^{0} \nu_{\tau}$ & 84 & $0.000038 \pm 0.000009$ \\
\hline$\tau^{-} \rightarrow K^{-} 2 \pi^{-} 2 \pi^{+} \pi^{0} \nu_{\tau}$ & 89 & $0.000001 \pm 0.000001$ \\
\hline$\tau^{-} \rightarrow \pi^{-} f_{1}\left(\rightarrow 2 \pi^{-} 2 \pi^{+}\right) \nu_{\tau}$ & 233 & $0.000052 \pm 0.000004$ \\
\hline
\end{tabular}




\begin{tabular}{lcc}
\hline \hline Decay mode & TauBBMode & BF \\
\hline$\tau^{-} \rightarrow \pi^{-} f_{1}\left(\rightarrow \pi^{-} \pi^{+} \eta(\rightarrow \gamma \gamma)\right) \nu_{\tau}$ & 253 & $0.000050 \pm 0.000003$ \\
\hline$\tau^{-} \rightarrow \pi^{-} f_{1}\left(\rightarrow \pi^{-} \pi^{+} \eta\left(\rightarrow 3 \pi^{0}\right)\right) \nu_{\tau}$ & 254 & $0.000041 \pm 0.000003$ \\
\hline$\tau^{-} \rightarrow \pi^{-} f_{1}\left(\rightarrow \pi^{-} \pi^{+} \eta\left(\rightarrow \pi^{-} \pi^{+} \pi^{0}\right)\right) \nu_{\tau}$ & 255 & $0.000029 \pm 0.000002$ \\
\hline$\tau^{-} \rightarrow \pi^{-} f_{1}\left(\rightarrow \pi^{-} \pi^{+} \eta\left(\rightarrow \pi^{-} \pi^{+} \gamma\right)\right) \nu_{\tau}$ & 256 & $0.000006 \pm 0.000001$ \\
\hline$\tau^{-} \rightarrow \pi^{-} 2 \pi^{0} \eta(\rightarrow \gamma \gamma) \nu_{\tau}$ & 37 & $0.000077 \pm 0.000015$ \\
\hline$\tau^{-} \rightarrow \pi^{-} 2 \pi^{0} \eta\left(\rightarrow \pi^{0} \pi^{0} \pi^{0}\right) \nu_{\tau}$ & 38 & $0.000064 \pm 0.000012$ \\
\hline$\tau^{-} \rightarrow \pi^{-} 2 \pi^{0} \eta\left(\rightarrow \pi^{-} \pi^{+} \pi^{0}\right) \nu_{\tau}$ & 39 & $0.000044 \pm 0.000009$ \\
\hline$\tau^{-} \rightarrow \pi^{-} 2 \pi^{0} \eta\left(\rightarrow \pi^{-} \pi^{+} \gamma\right) \nu_{\tau}$ & 40 & $0.000010 \pm 0.000002$ \\
\hline \hline
\end{tabular}

Table 7.2: List of 92 initialized $\tau^{-}$decay modes with their BF.

\subsection{Modelling of visible mass in $\tau$ decays}

Validation of the modeling of visible mass $m_{v i s}$ distribution for $92 \tau^{-}$decays whose branching fractions add up to unity are presented in Figures 7.1 through 7.92 [72]. They are obtained by generating 92 different samples of 10000 events with JAK1 and JAK2 parameters set to the TauBBMode value listed in Table 7.2.

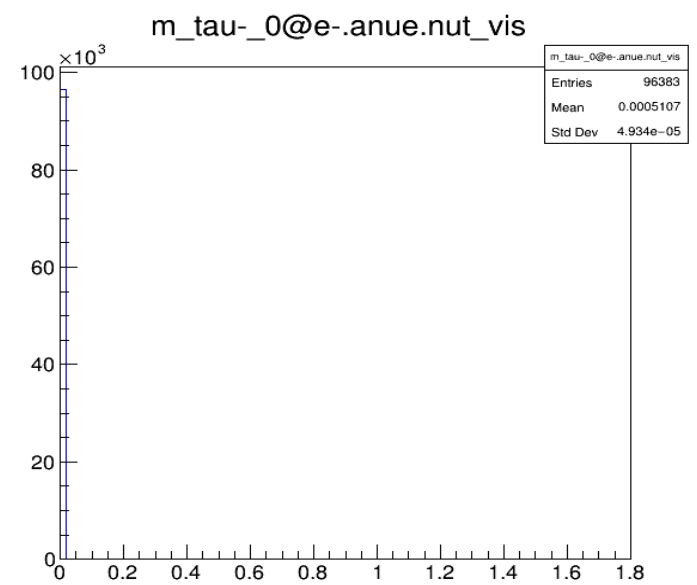

Figure 7.1: $m_{v i s}$ for $\tau^{-} \rightarrow e^{-} \bar{\nu}_{e} \nu_{\tau}$

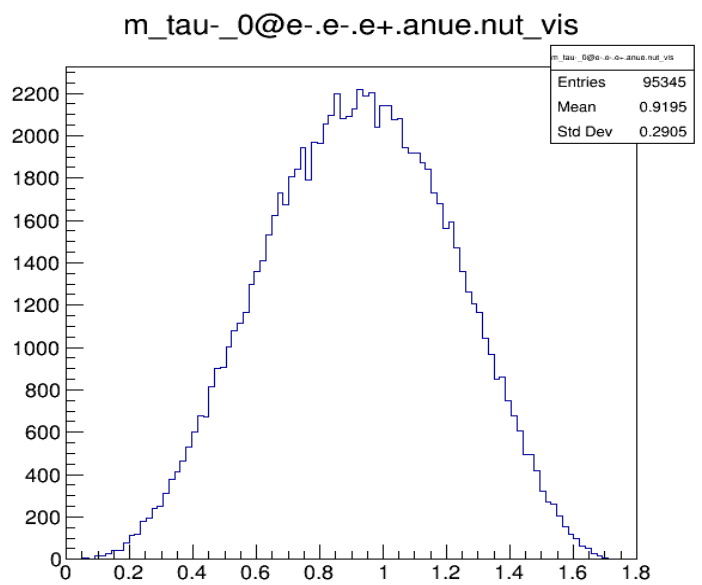

Figure 7.2: $m_{v i s}$ for $\tau^{-} \rightarrow e^{-} e^{-} e^{+} \bar{\nu}_{e} \nu_{\tau}$ 
m_tau-_0@mu-.anumu.nut_vis

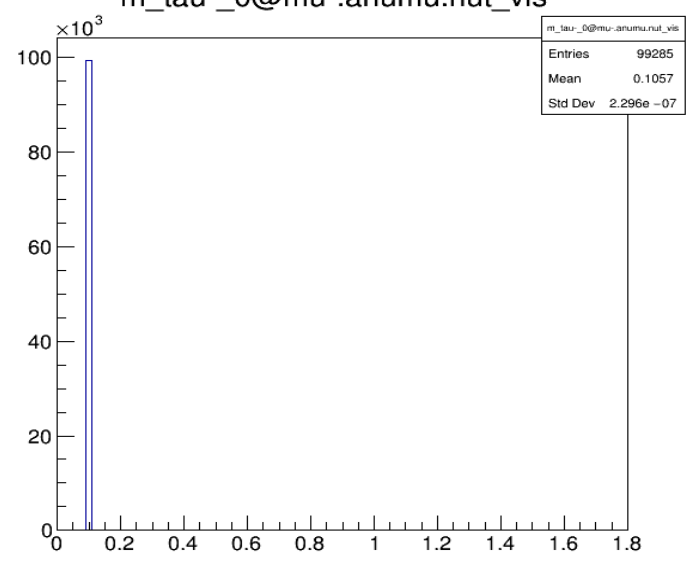

Figure 7.3: $m_{v i s}$ for $\tau^{-} \rightarrow \mu^{-} \bar{\nu}_{\mu} \nu_{\tau}$

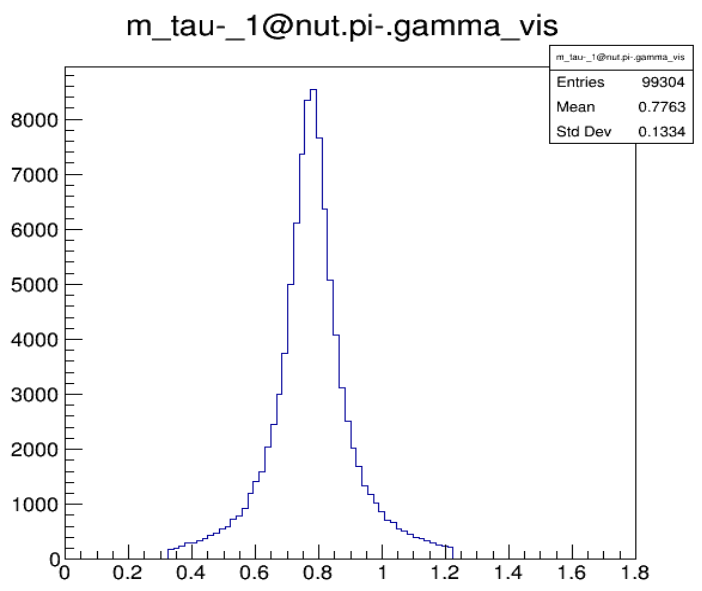

Figure 7.5: $m_{v i s}$ for $\tau^{-} \rightarrow \rho^{-}\left(\rightarrow \pi^{-} \gamma\right) \nu_{\tau}$

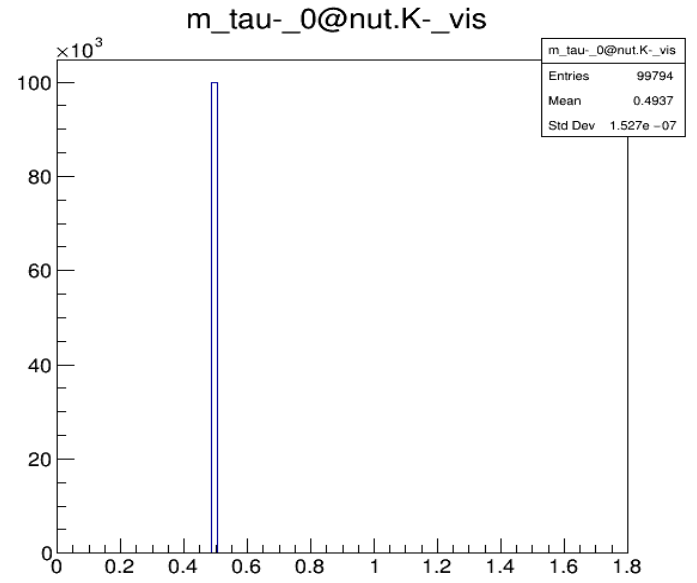

Figure 7.7: $m_{v i s}$ for $\tau^{-} \rightarrow K^{-} \nu_{\tau}$

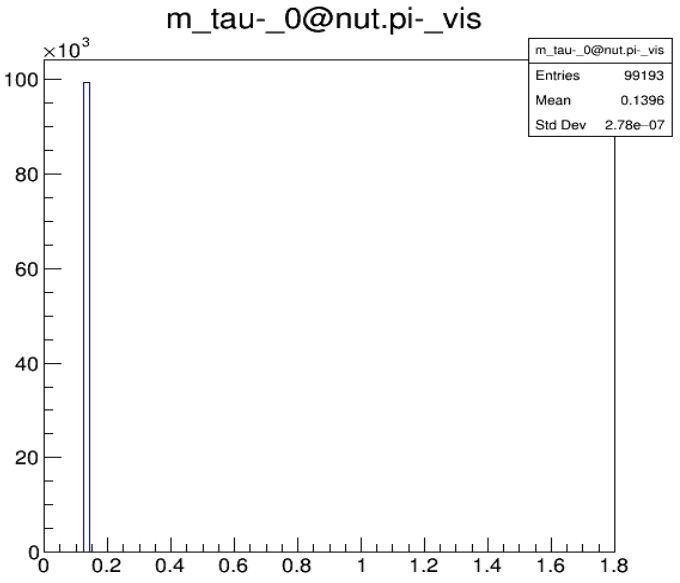

Figure 7.4: $m_{v i s}$ for $\tau^{-} \rightarrow \pi^{-} \nu_{\tau}$

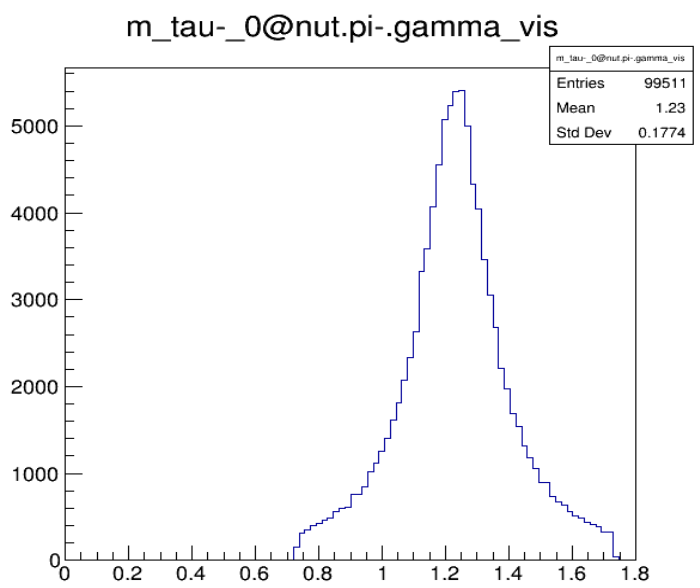

Figure 7.6: $m_{v i s}$ for $\tau^{-} \rightarrow a_{1}^{-}\left(\rightarrow \pi^{-} \gamma\right) \nu_{\tau}$

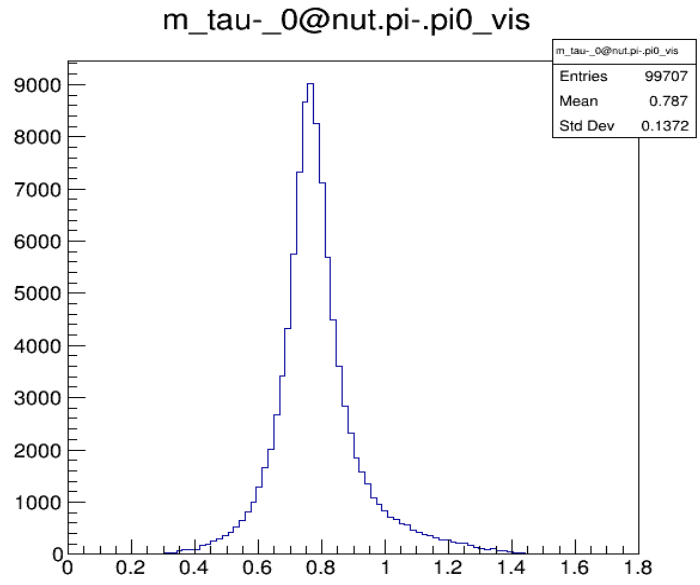

Figure 7.8: $m_{v i s}$ for $\tau^{-} \rightarrow \pi^{-} \pi^{0} \nu_{\tau}$ 
m tau-1@nut.pi-.pi0.gamma vis

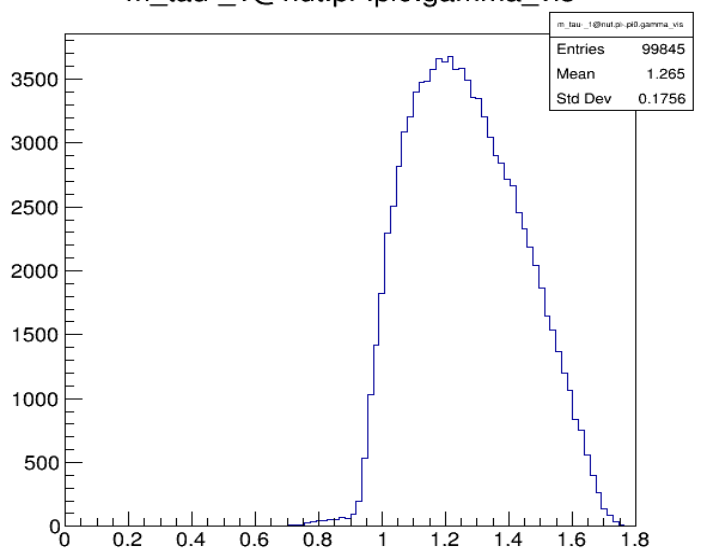

Figure 7.9: $m_{v i s}$ for $\tau^{-} \rightarrow \pi^{-} \omega(\rightarrow$ $\left.\pi^{0} \gamma\right) \nu_{\tau}$

m_tau-_0@nut.pi-.pi0.pi0_vis

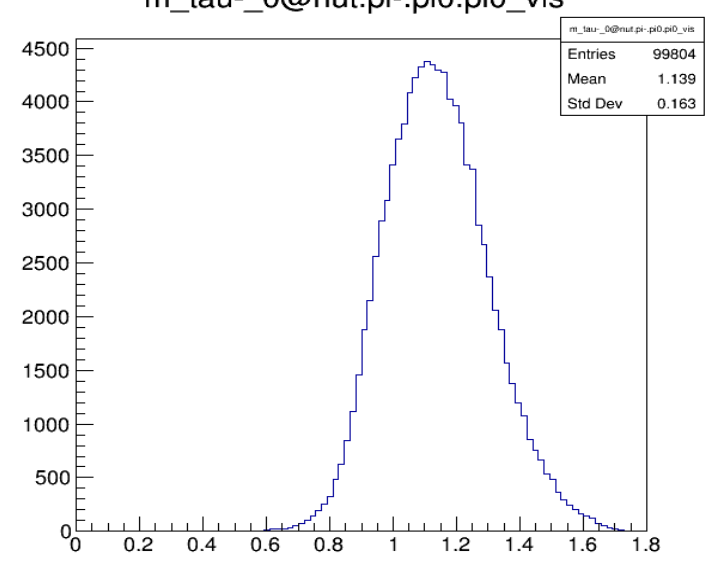

Figure 7.11: $m_{v i s}$ for $\tau^{-} \rightarrow \pi^{-} 2 \pi^{0} \nu_{\tau}$

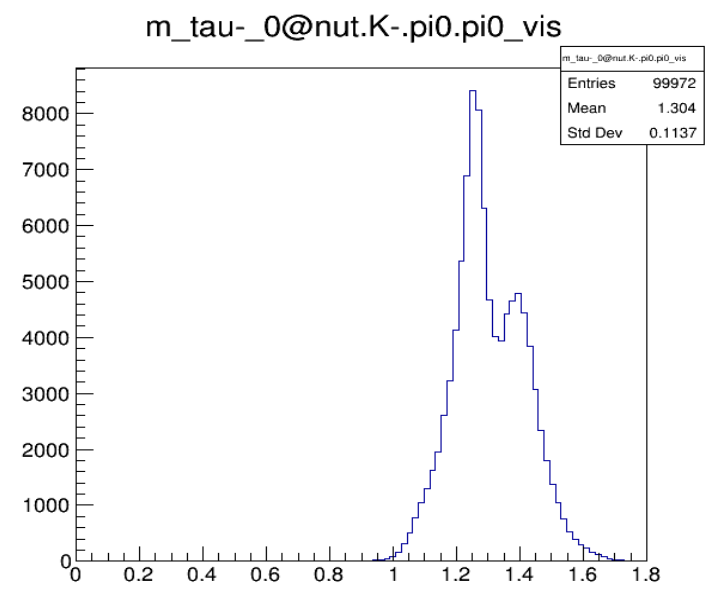

Figure 7.13: $m_{v i s}$ for $\tau^{-} \rightarrow K^{-} 2 \pi^{0} \nu_{\tau}$

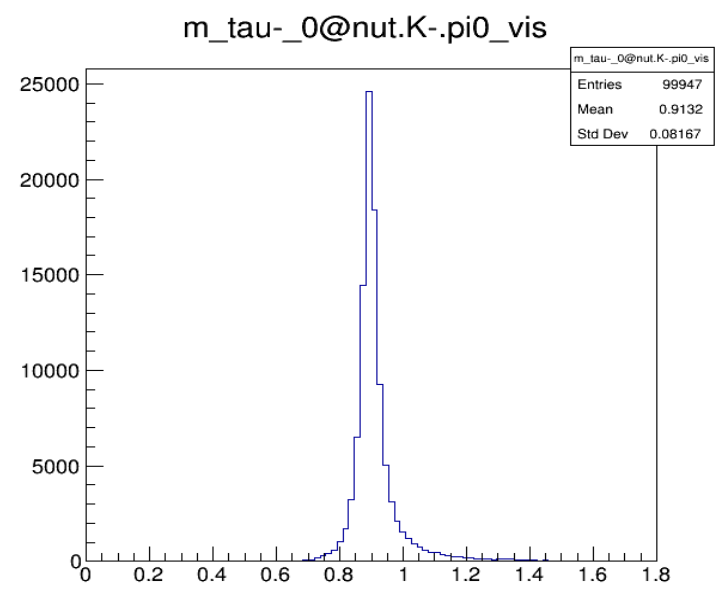

Figure 7.10: $m_{v i s}$ for $\tau^{-} \rightarrow K^{-} \pi^{0} \nu_{\tau}$

m_tau-_0@nut.pi-.pi0.pi0.gamma_vis

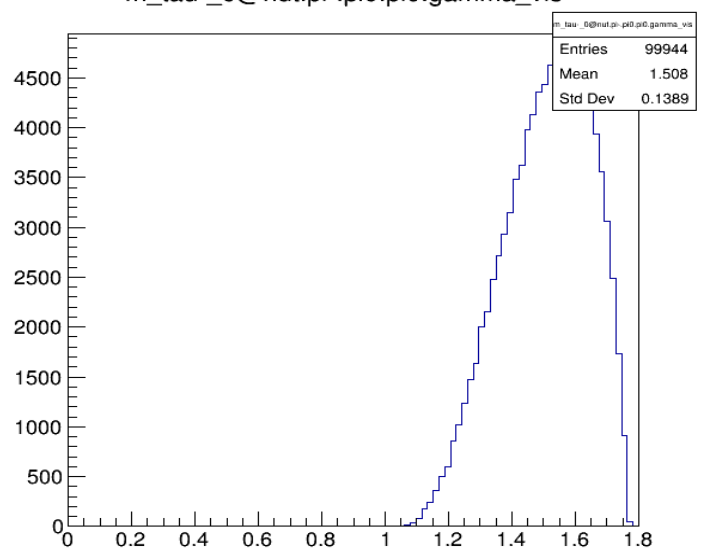

Figure 7.12: $m_{v i s}$ for $\tau^{-} \rightarrow \pi^{-} \pi^{0} \omega(\rightarrow$ $\left.\pi^{0} \gamma\right) \nu_{\tau}$

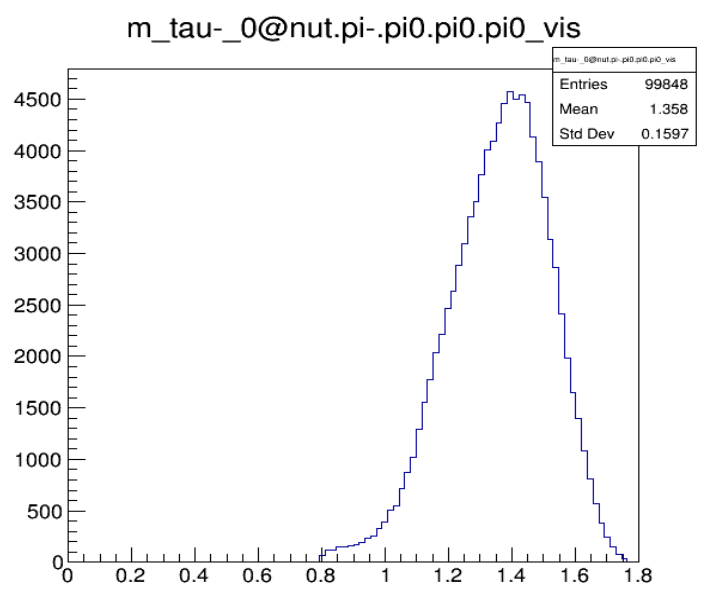

Figure 7.14: $m_{v i s}$ for $\tau^{-} \rightarrow \pi^{-} 3 \pi^{0} \nu_{\tau}$ 


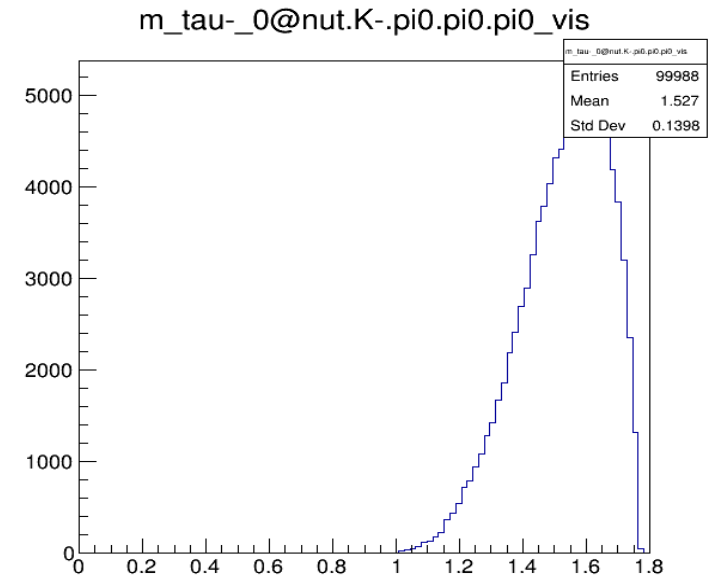

Figure 7.15: $m_{v i s}$ for $\tau^{-} \rightarrow K^{-} 3 \pi^{0} \nu_{\tau}$

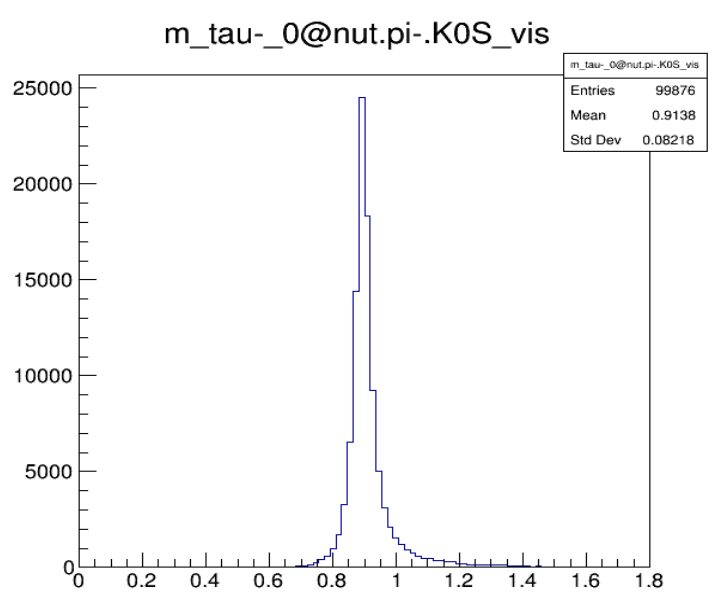

Figure 7.17: $m_{v i s}$ for $\tau^{-} \rightarrow \pi^{-} K_{S}^{0} \nu_{\tau}$

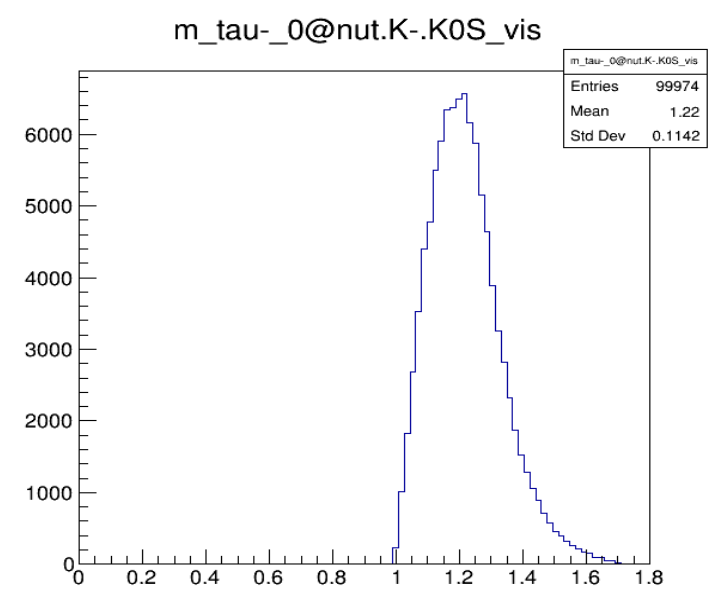

Figure 7.19: $m_{v i s}$ for $\tau^{-} \rightarrow K^{-} K_{S}^{0} \nu_{\tau}$

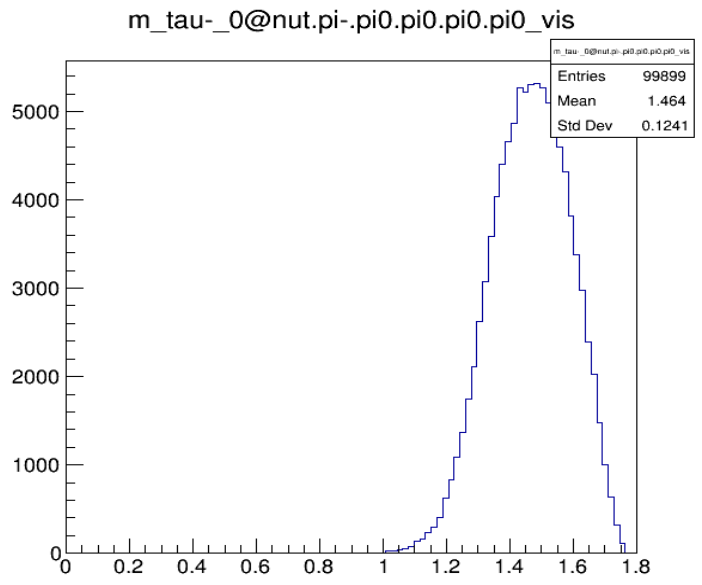

Figure 7.16: $m_{v i s}$ for $\tau^{-} \rightarrow \pi^{-} 4 \pi^{0} \nu_{\tau}$

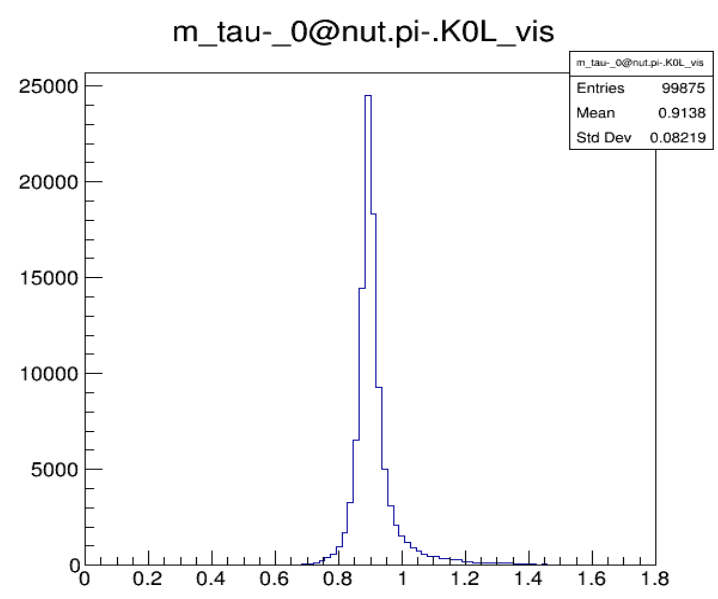

Figure 7.18: $m_{v i s}$ for $\tau^{-} \rightarrow \pi^{-} K_{L}^{0} \nu_{\tau}$

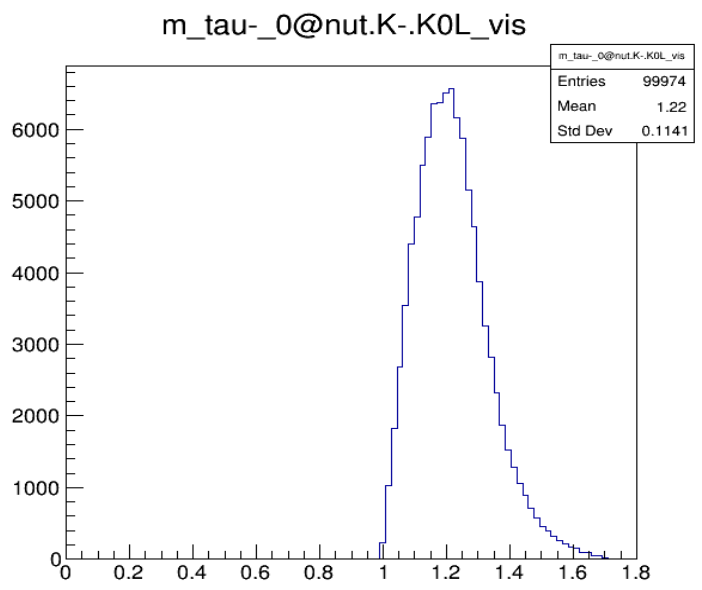

Figure 7.20: $m_{v i s}$ for $\tau^{-} \rightarrow K^{-} K_{L}^{0} \nu_{\tau}$ 
m_tau-_0@nut.pi-.pi0.K0S_vis

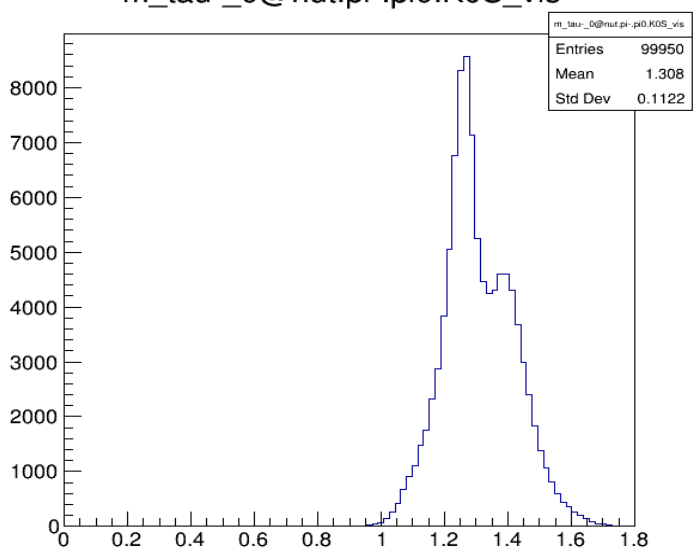

Figure 7.21: $m_{v i s}$ for $\tau^{-} \rightarrow \pi^{-} K_{S}^{0} \pi^{0} \nu_{\tau}$

m_tau-_0@nut.K-.pi0.KoS_vis

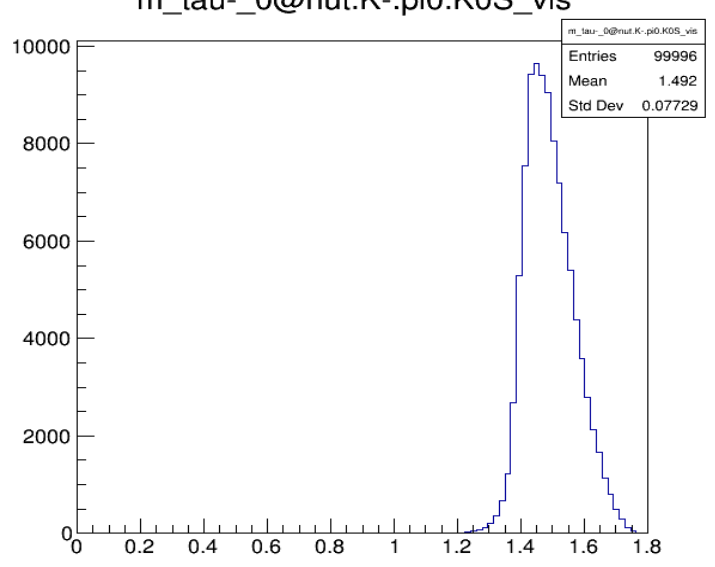

Figure 7.23: $m_{v i s}$ for $\tau^{-} \rightarrow K^{-} \pi^{0} K_{S}^{0} \nu_{\tau}$

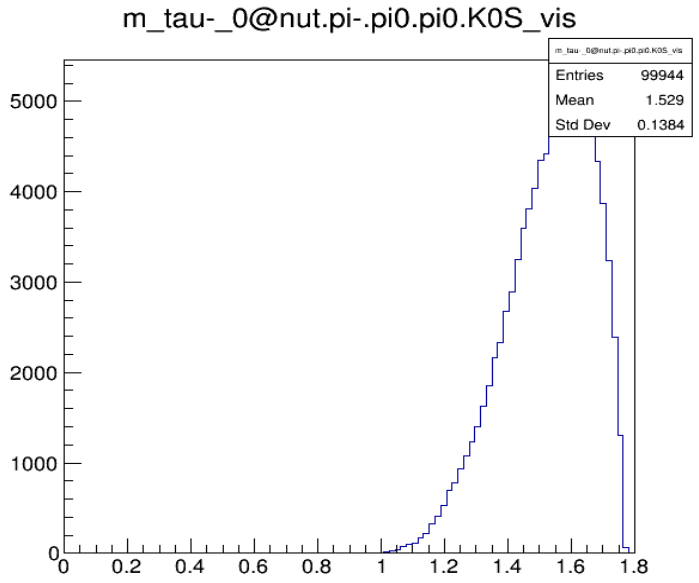

Figure 7.25: $m_{v i s}$ for $\tau^{-} \rightarrow \pi^{-} 2 \pi^{0} K_{S}^{0} \nu_{\tau}$

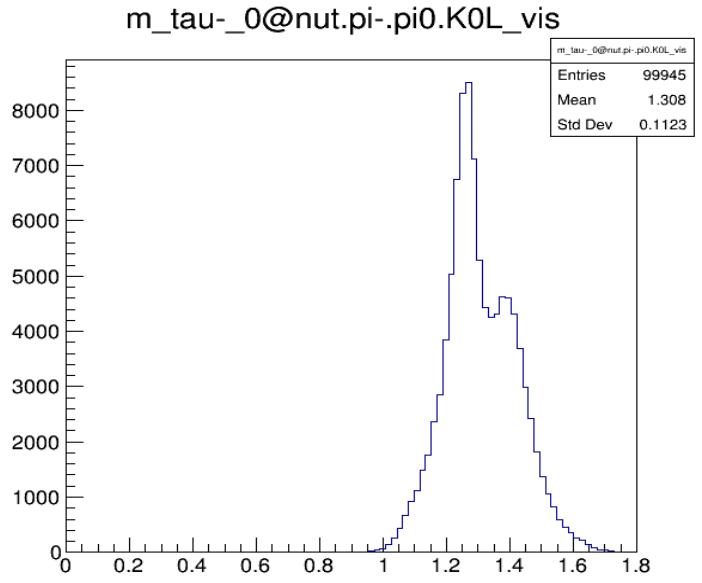

Figure 7.22: $m_{v i s}$ for $\tau^{-} \rightarrow \pi^{-} K_{L}^{0} \pi^{0} \nu_{\tau}$

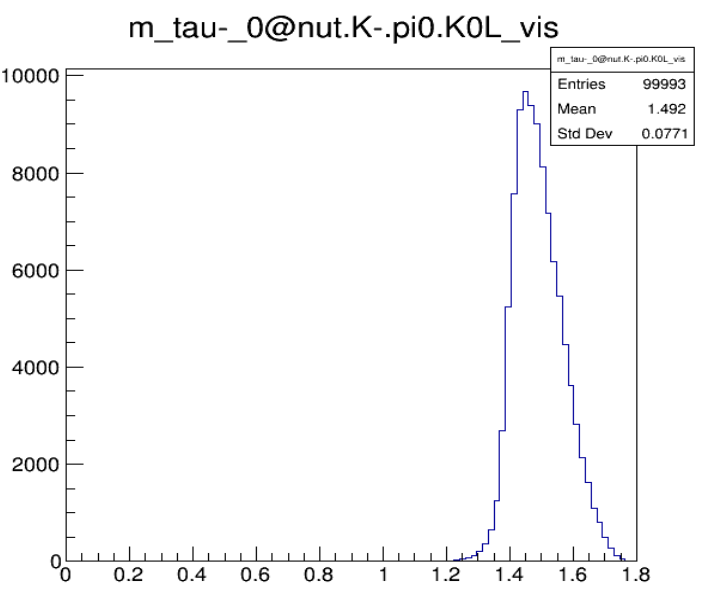

Figure 7.24: $m_{v i s}$ for $\tau^{-} \rightarrow K^{-} \pi^{0} K_{L}^{0} \nu_{\tau}$

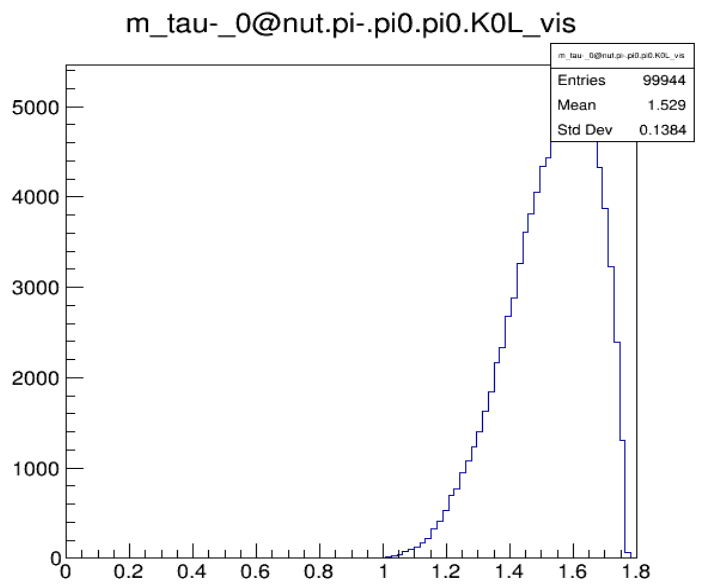

Figure 7.26: $m_{v i s}$ for $\tau^{-} \rightarrow \pi^{-} 2 \pi^{0} K_{L}^{0} \nu_{\tau}$ 


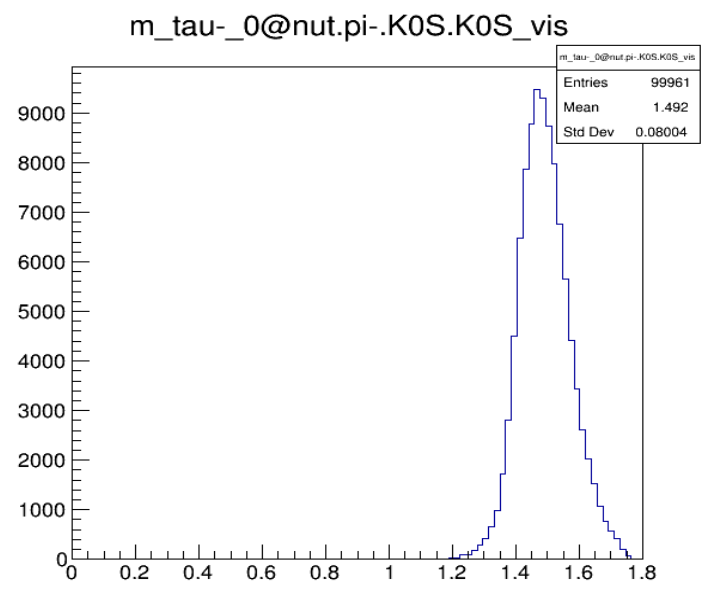

Figure 7.27: $m_{v i s}$ for $\tau^{-} \rightarrow \pi^{-} K_{S}^{0} K_{S}^{0} \nu_{\tau}$

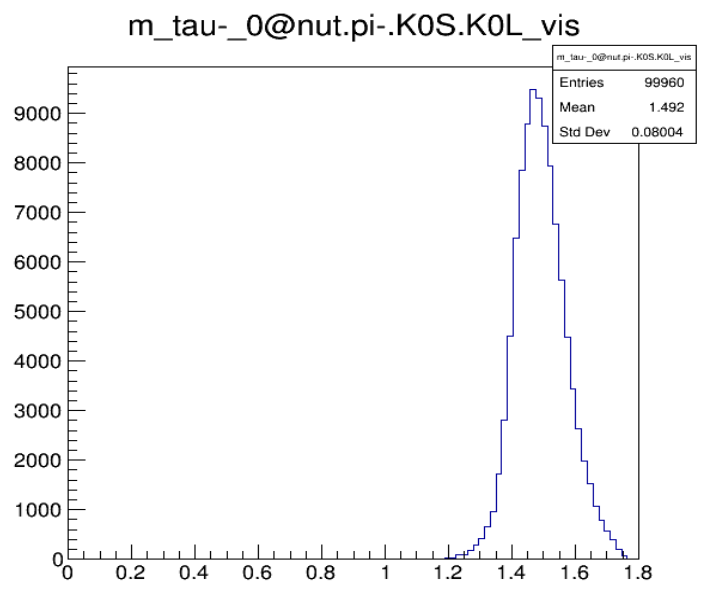

Figure 7.29: $m_{v i s}$ for $\tau^{-} \rightarrow \pi^{-} K_{S}^{0} K_{L}^{0} \nu_{\tau}$

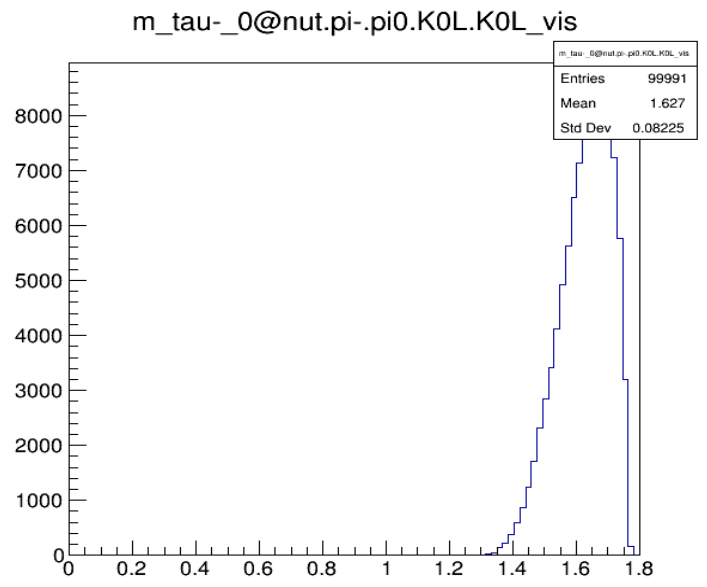

Figure 7.31: $m_{v i s}$ for $\tau^{-} \rightarrow$ Figure 7.32: $m_{v i s}$ for $\tau^{-} \rightarrow$ $\pi^{-} \pi^{0} K_{L}^{0} K_{L}^{0} \nu_{\tau}$

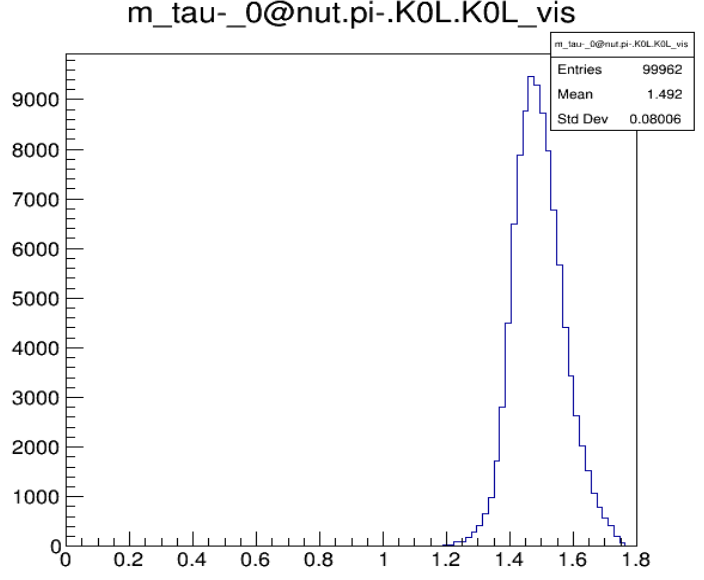

Figure 7.28: $m_{v i s}$ for $\tau^{-} \rightarrow \pi^{-} K_{L}^{0} K_{L}^{0} \nu_{\tau}$

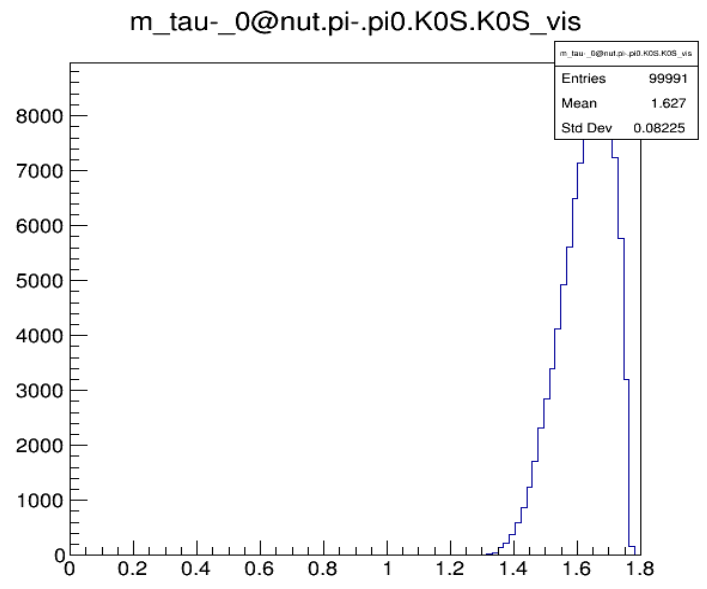

Figure 7.30: $m_{v i s}$ for $\tau^{-} \quad \rightarrow$ $\pi^{-} \pi^{0} K_{S}^{0} K_{S}^{0} \nu_{\tau}$

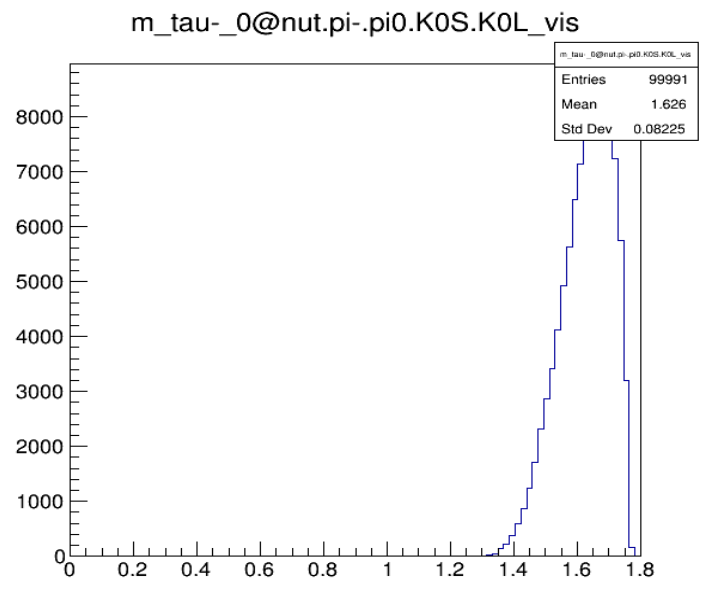

$\pi^{-} \pi^{0} K_{S}^{0} K_{L}^{0} \nu_{\tau}$

87 
m tau-1@nut.pi-.pio.gamma.gamma vis

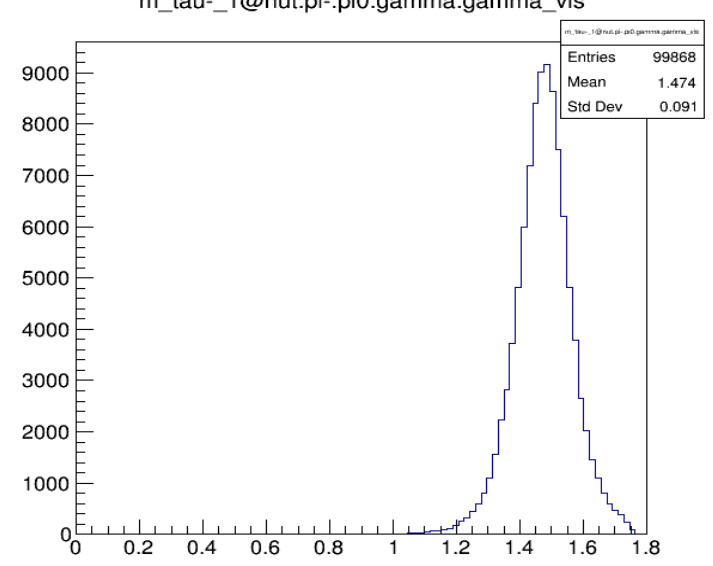

Figure 7.33: $m_{v i s}$ for $\tau^{-} \rightarrow \pi^{-} \pi^{0} \eta(\rightarrow$ $\gamma \gamma) \nu_{\tau}$

m_tau-_0@nut.pi-.pi-.pi+.pio.pio_vis

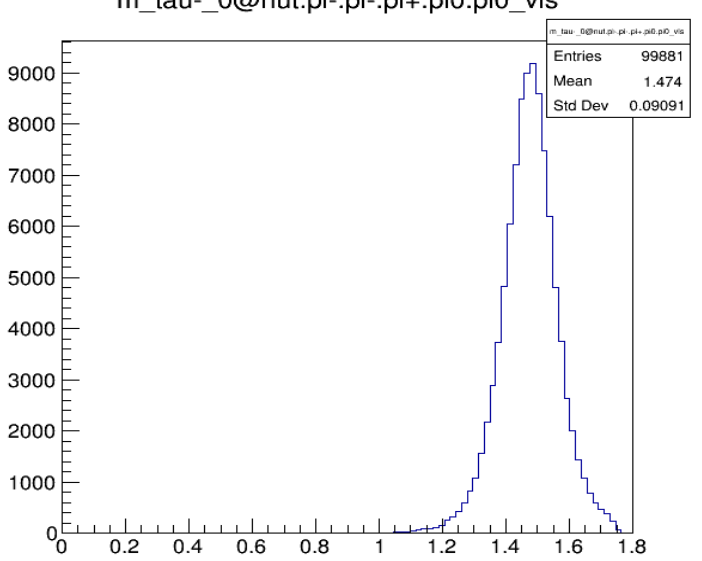

Figure 7.35: $m_{v i s}$ for $\tau^{-} \rightarrow \pi^{-} \pi^{0} \eta(\rightarrow$ $\left.\pi^{+} \pi^{-} \pi^{0}\right) \nu_{\tau}$

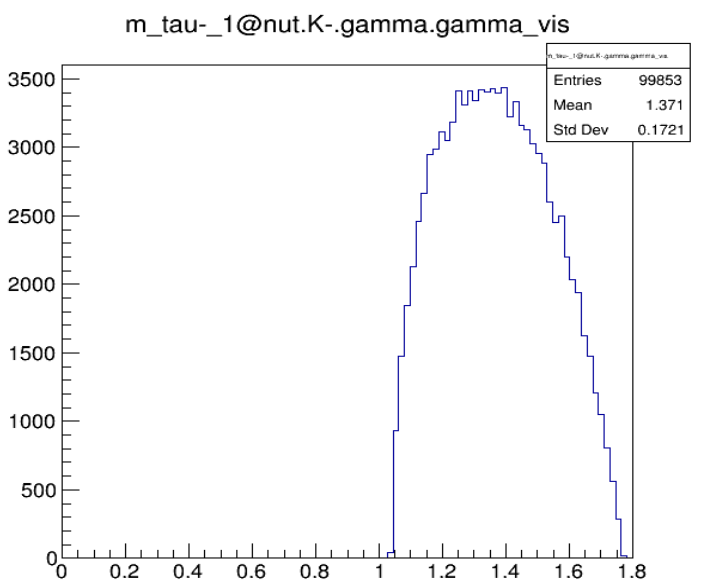

Figure 7.37: $m_{v i s}$ for $\tau^{-} \rightarrow K^{-} \eta(\rightarrow$ $\gamma \gamma) \nu_{\tau}$ m tau-0@nut.pi-.pi0.pi0.pi0.pi0_vis

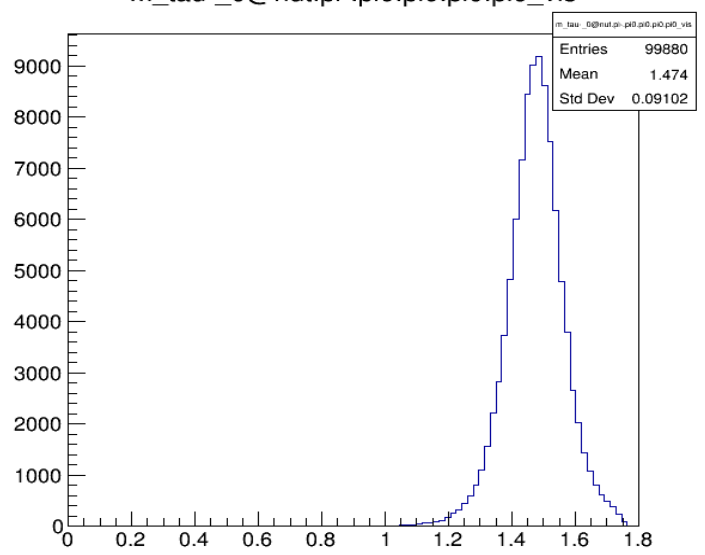

Figure 7.34: $m_{v i s}$ for $\tau^{-} \rightarrow \pi^{-} \pi^{0} \eta(\rightarrow$ $\left.\pi^{0} \pi^{0} \pi^{0}\right) \nu_{\tau}$

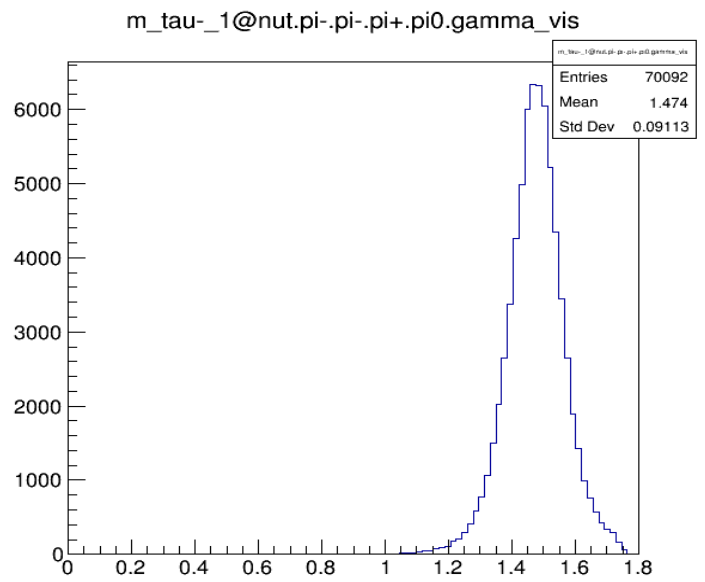

Figure 7.36: $m_{v i s}$ for $\tau^{-} \rightarrow \pi^{-} \pi^{0} \eta(\rightarrow$ $\left.\pi^{+} \pi^{-} \gamma\right) \nu_{\tau}$

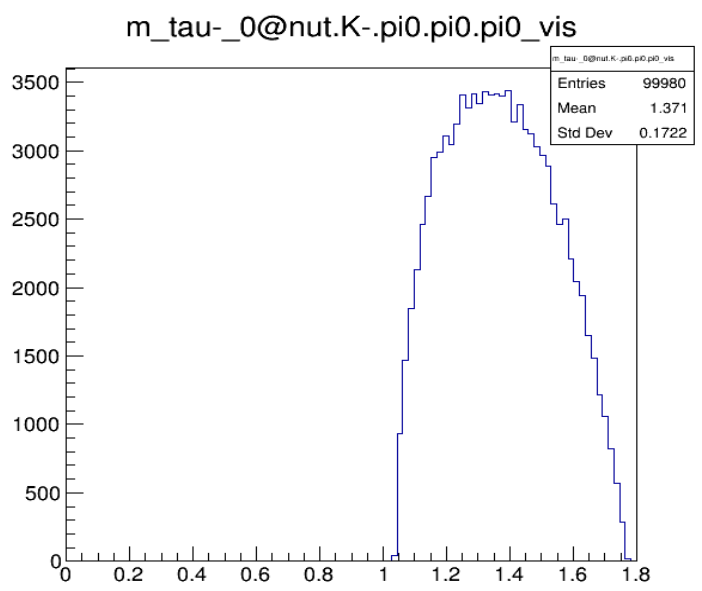

Figure 7.38: $m_{\text {vis }}$ for $\tau^{-} \rightarrow K^{-} \eta(\rightarrow$ $\left.\pi^{0} \pi^{0} \pi^{0}\right) \nu_{\tau}$ 
m_tau-_0@nut.pi-.pi+.K-.pio_vis

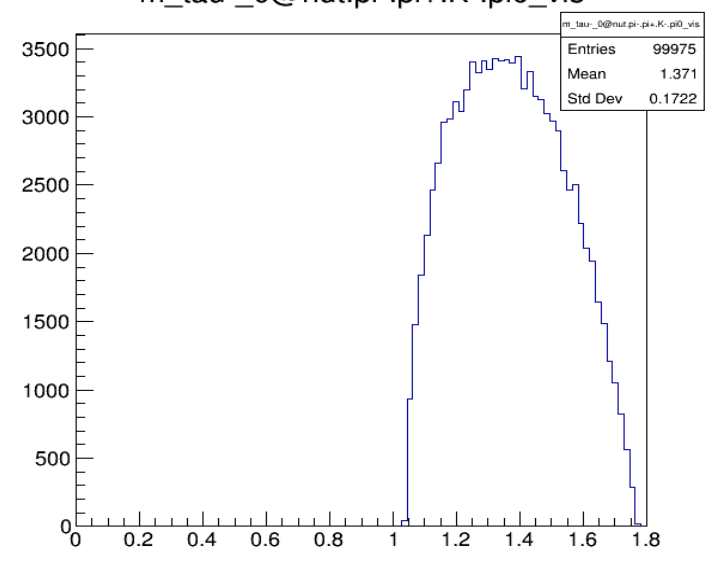

Figure 7.39: $m_{v i s}$ for $\tau^{-} \rightarrow K^{-} \eta(\rightarrow$ $\left.\pi^{+} \pi^{-} \pi^{0}\right) \nu_{\tau}$

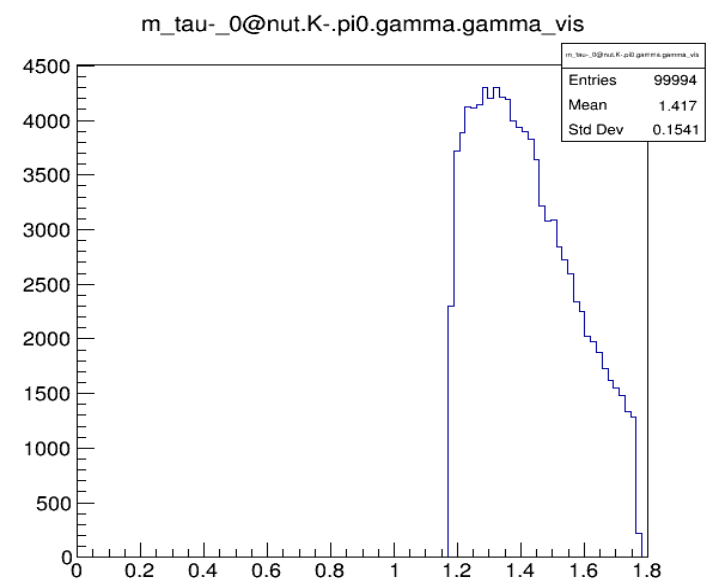

Figure 7.41: $m_{v i s}$ for $\tau^{-} \rightarrow K^{-} \pi^{0} \eta(\rightarrow$ $\gamma \gamma) \nu_{\tau}$

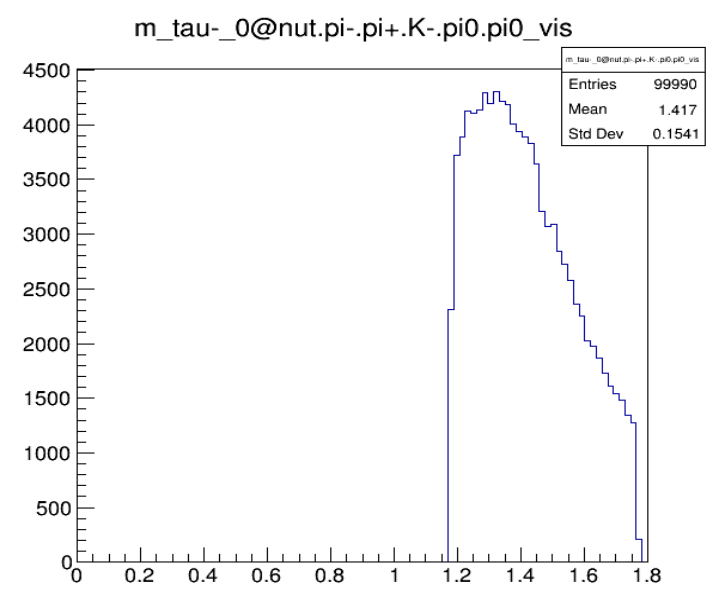

Figure 7.43: $m_{v i s}$ for $\tau^{-} \rightarrow K^{-} \pi^{0} \eta(\rightarrow$ $\left.\pi^{+} \pi^{-} \pi^{0}\right) \nu_{\tau}$

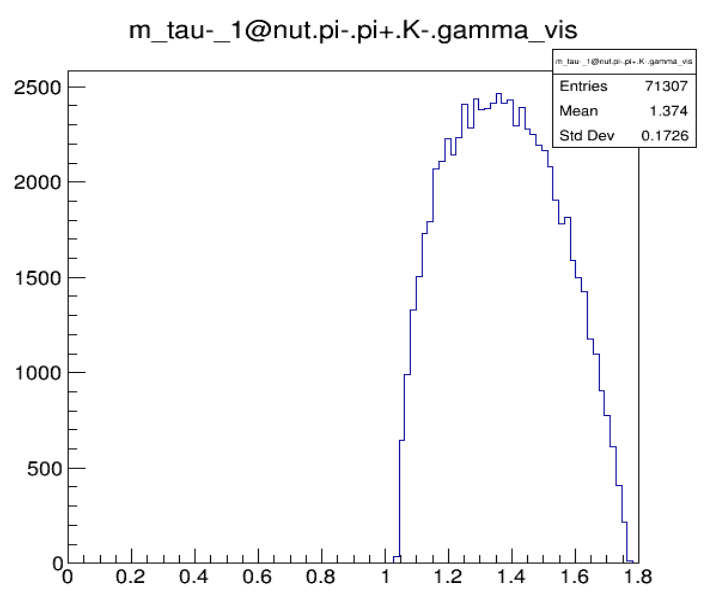

Figure 7.40: $m_{v i s}$ for $\tau^{-} \rightarrow K^{-} \eta(\rightarrow$ $\left.\pi^{+} \pi^{-} \gamma\right) \nu_{\tau}$

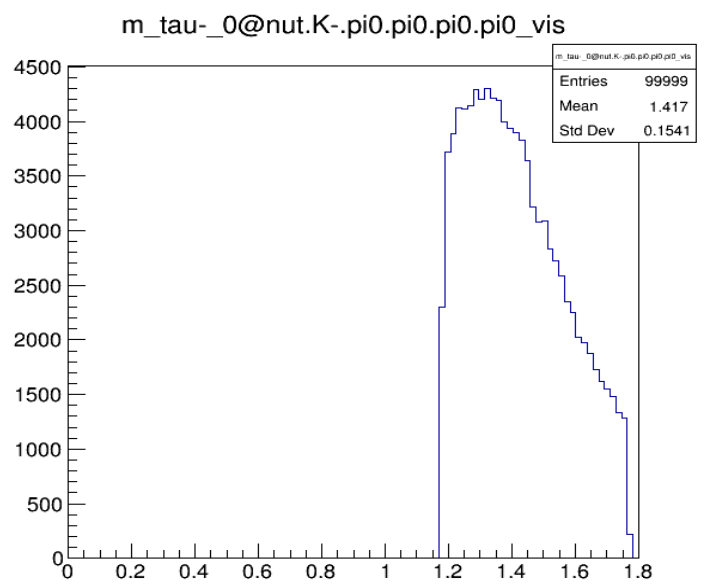

Figure 7.42: $m_{v i s}$ for $\tau^{-} \rightarrow K^{-} \pi^{0} \eta(\rightarrow$ $\left.\pi^{0} \pi^{0} \pi^{0}\right) \nu_{\tau}$

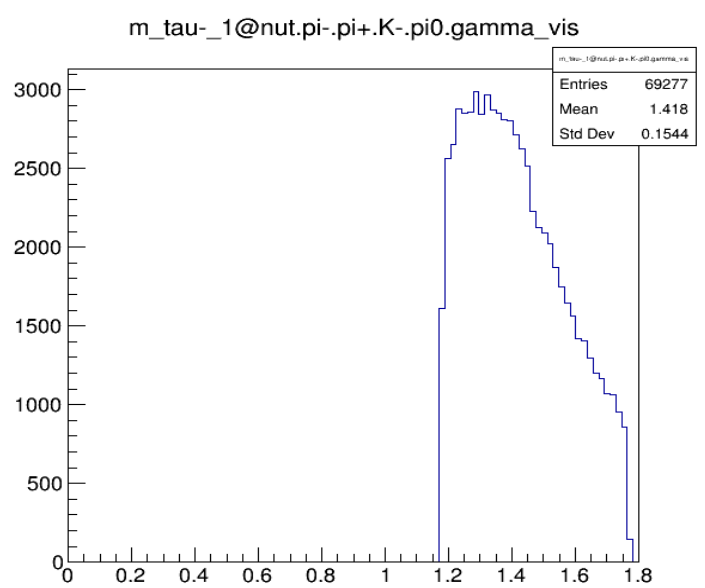

Figure 7.44: $m_{v i s}$ for $\tau^{-} \rightarrow K^{-} \pi^{0} \eta(\rightarrow$ $\left.\pi^{+} \pi^{-} \gamma\right) \nu_{\tau}$ 
m_tau-_0@nut.pi-.KoS.gamma.gamma_vis

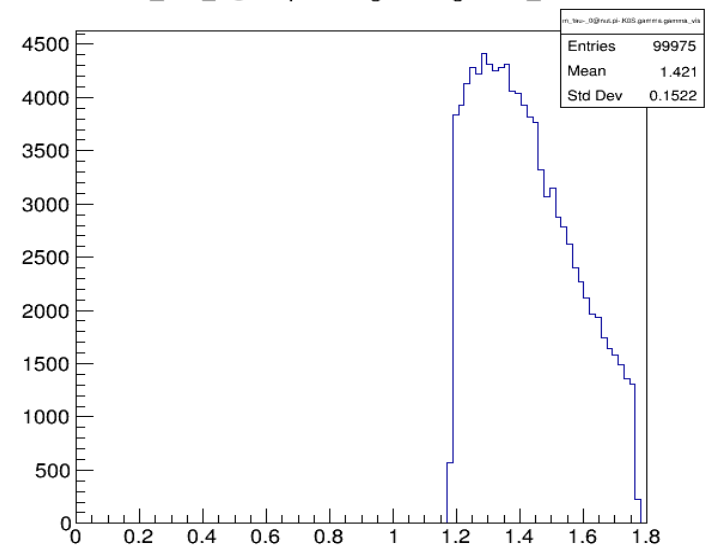

Figure 7.45: $m_{v i s}$ for $\tau^{-} \rightarrow \pi^{-} K_{S}^{0} \eta(\rightarrow$ $\gamma \gamma) \nu_{\tau}$

m_tau-_0@nut.pi-.pi-.pi+.pi0.KoS_vis

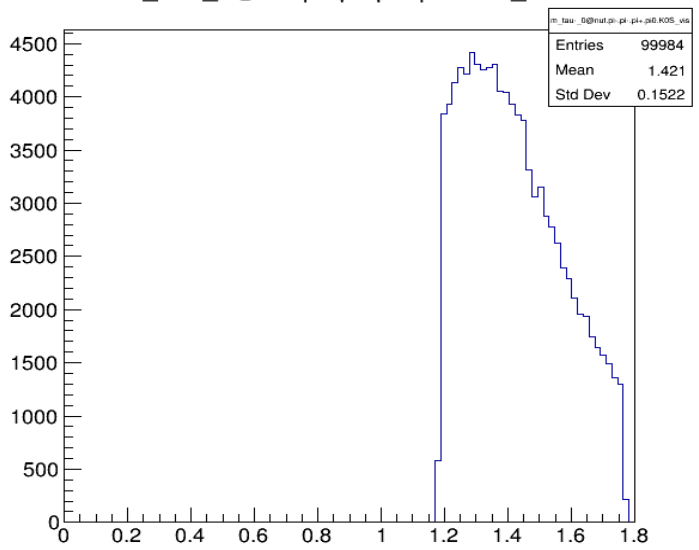

Figure 7.47: $m_{v i s}$ for $\tau^{-} \rightarrow \pi^{-} K_{S}^{0} \eta(\rightarrow$ $\left.\pi^{+} \pi^{-} \pi^{0}\right) \nu_{\tau}$

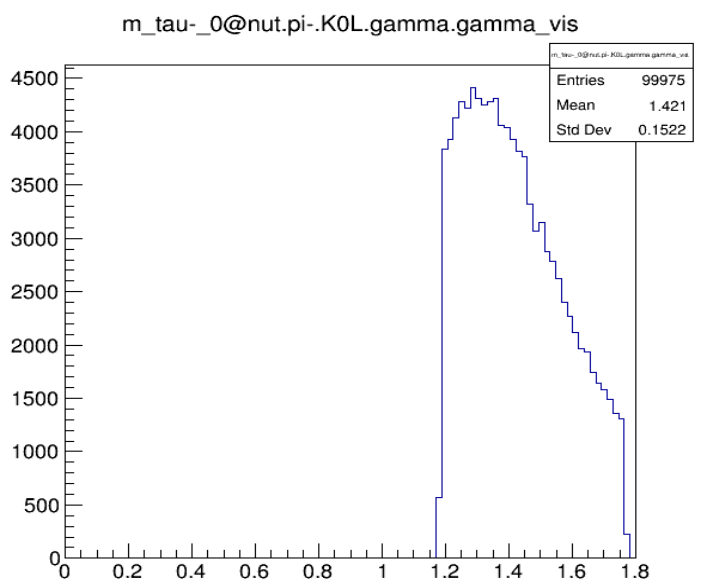

Figure 7.49: $m_{v i s}$ for $\tau^{-} \rightarrow \pi^{-} K_{L}^{0} \eta(\rightarrow$ $\gamma \gamma) \nu_{\tau}$ m tau-0@nut.pi-.pi0.pi0.pi0.Kos vis

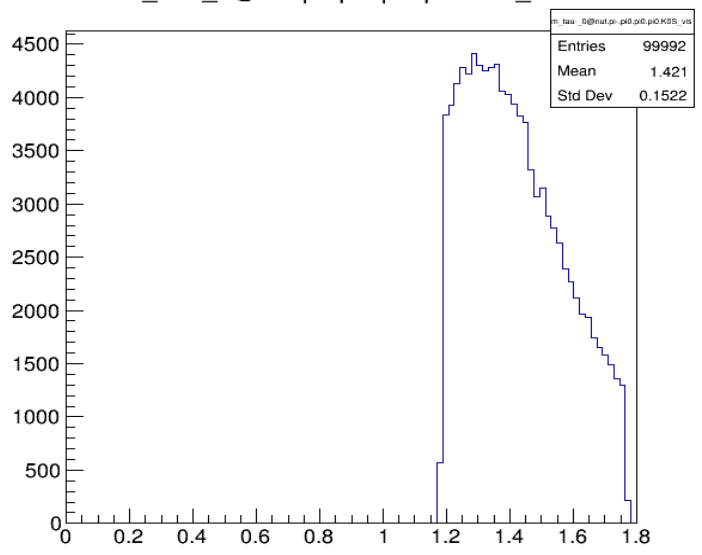

Figure 7.46: $m_{v i s}$ for $\tau^{-} \rightarrow \pi^{-} K_{S}^{0} \eta(\rightarrow$ $\left.\pi^{0} \pi^{0} \pi^{0}\right) \nu_{\tau}$

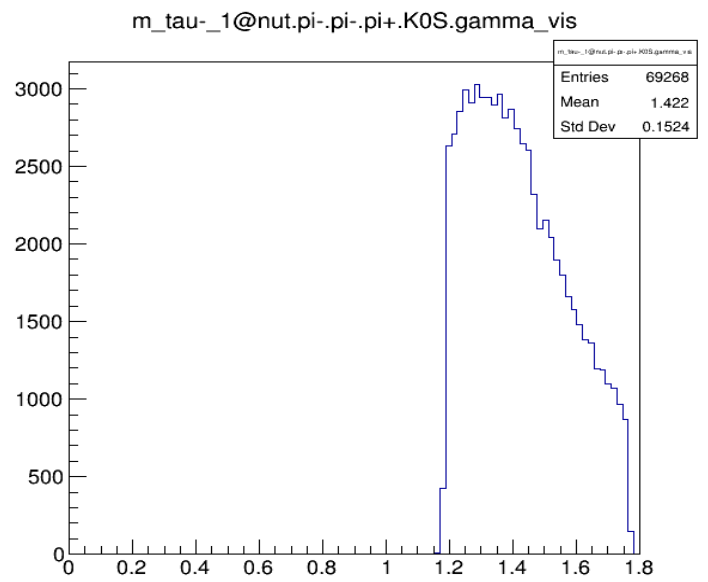

Figure 7.48: $m_{v i s}$ for $\tau^{-} \rightarrow \pi^{-} K_{S}^{0} \eta(\rightarrow$ $\left.\pi^{+} \pi^{-} \gamma\right) \nu_{\tau}$

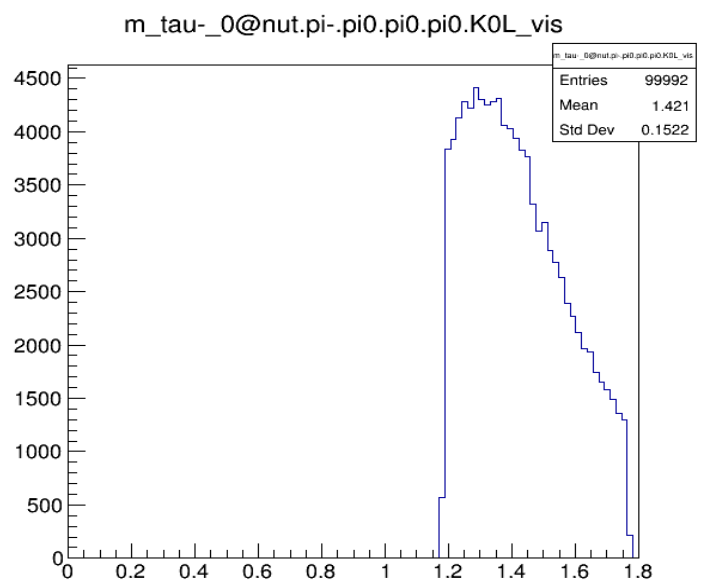

Figure 7.50: $m_{v i s}$ for $\tau^{-} \rightarrow \pi^{-} K_{L}^{0} \eta(\rightarrow$ $\left.\pi^{0} \pi^{0} \pi^{0}\right) \nu_{\tau}$ 
m tau-0@nut.pi-.pi-.pi+.pi0.K0L vis

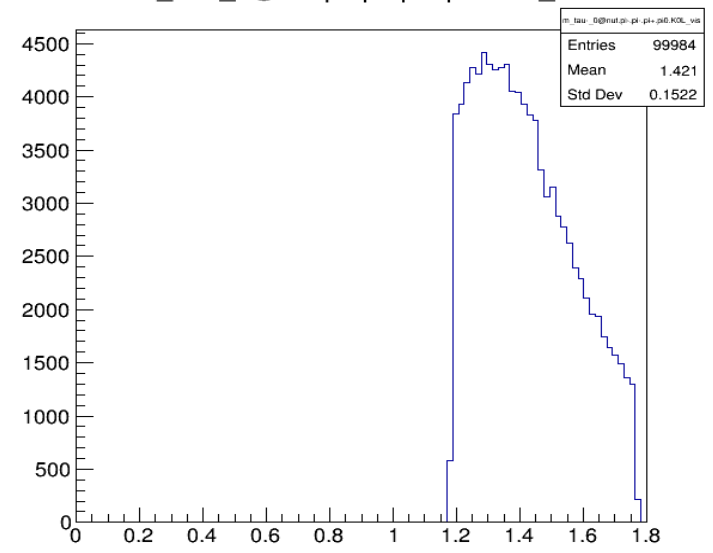

Figure 7.51: $m_{v i s}$ for $\tau^{-} \rightarrow \pi^{-} K_{L}^{0} \eta(\rightarrow$ $\left.\pi^{+} \pi^{-} \pi^{0}\right) \nu_{\tau}$

m_tau-_0@nut.pi-.pi-.pi+_vis

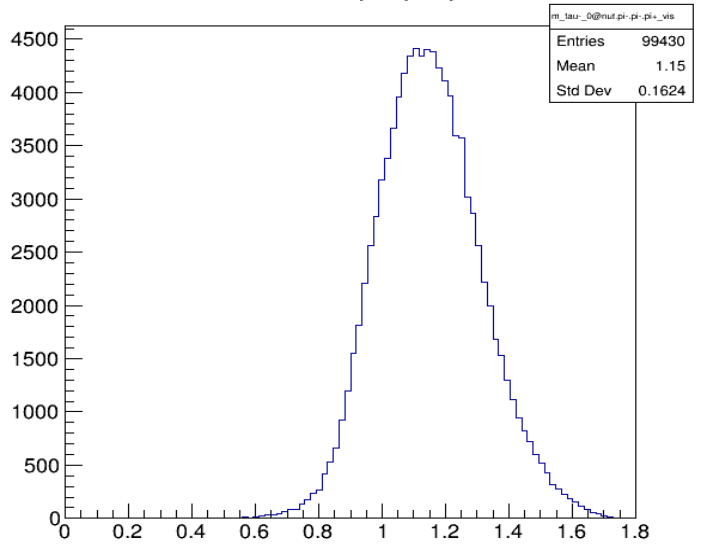

Figure 7.53: $m_{v i s}$ for $\tau^{-} \rightarrow 2 \pi^{-} \pi^{+} \nu_{\tau}$

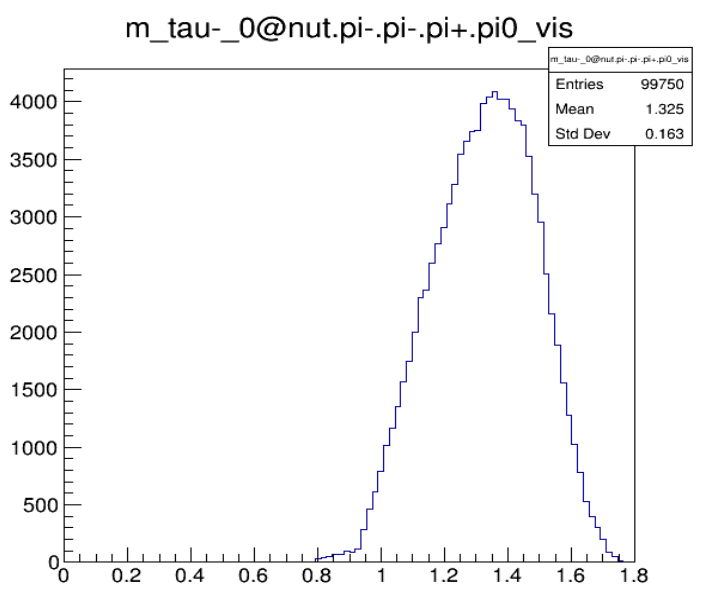

Figure 7.55: $m_{v i s}$ for $\tau^{-} \rightarrow 2 \pi^{-} \pi^{+} \pi^{0} \nu_{\tau}$

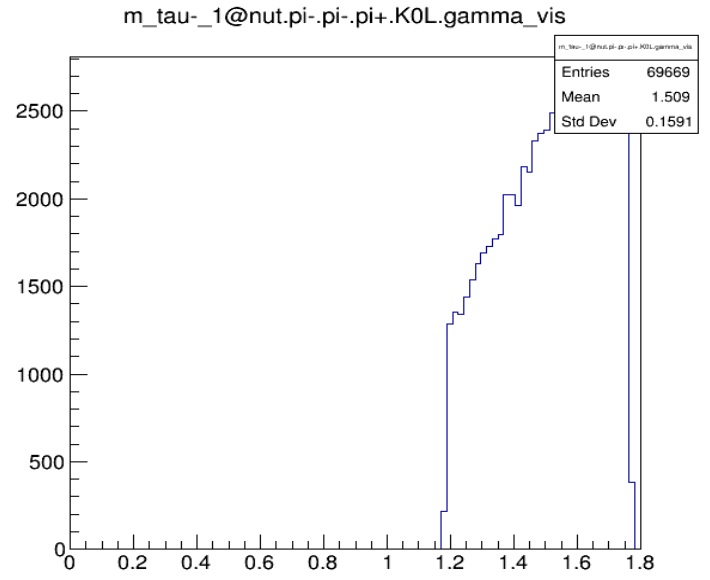

Figure 7.52: $m_{v i s}$ for $\tau^{-} \rightarrow \pi^{-} K_{L}^{0} \eta(\rightarrow$ $\left.\pi^{+} \pi^{-} \gamma\right) \nu_{\tau}$

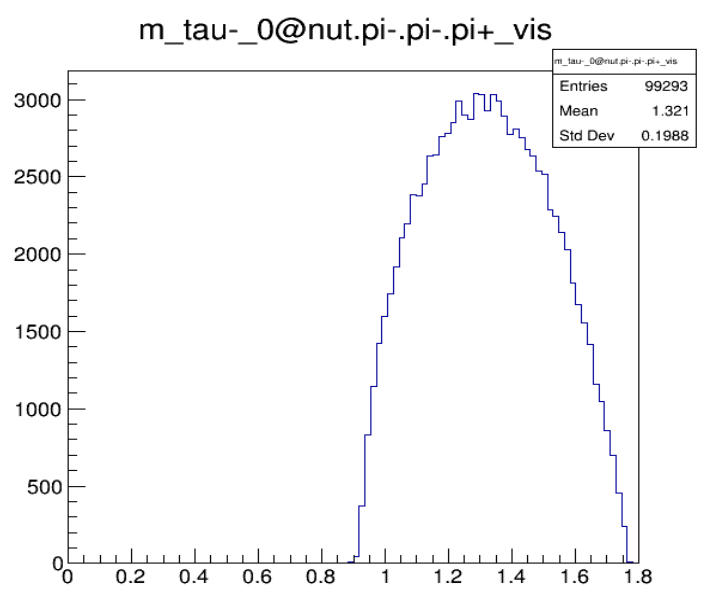

Figure 7.54: $m_{v i s}$ for $\tau^{-} \rightarrow \pi^{-} \omega(\rightarrow$ $\left.\pi^{+} \pi^{-}\right) \nu_{\tau}$

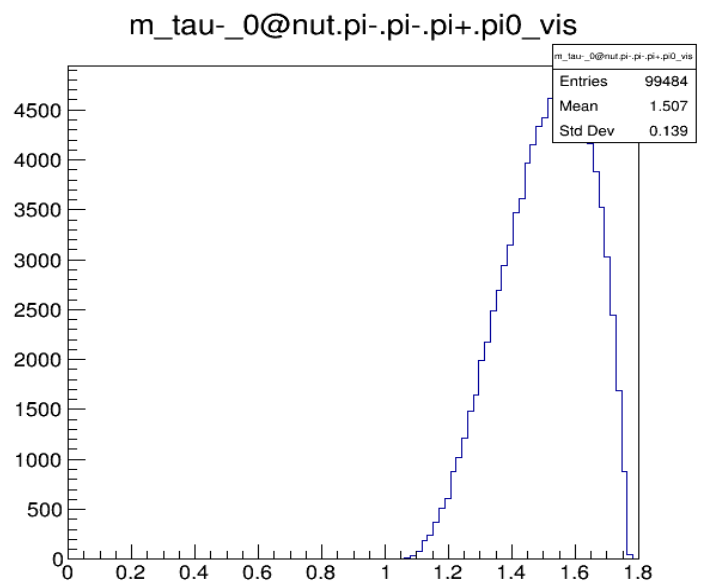

Figure 7.56: $m_{v i s}$ for $\tau^{-} \rightarrow \pi^{-} \pi^{0} \omega(\rightarrow$ $\left.\pi^{+} \pi^{-}\right) \nu_{\tau}$ 

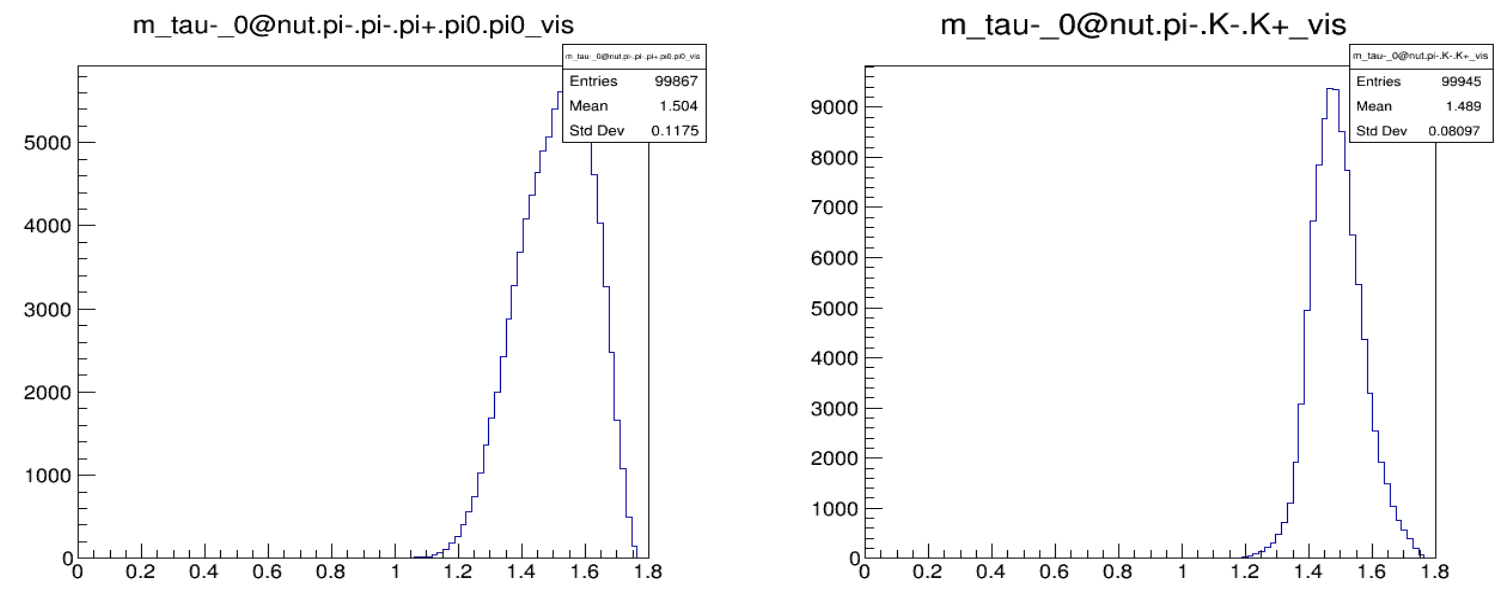

Figure 7.57: $m_{v i s}$ for $\tau^{-} \rightarrow 2 \pi^{-} \pi^{+} 2 \pi^{0} \nu_{\tau}$

Figure 7.58: $m_{v i s}$ for $\tau^{-} \rightarrow \pi^{-} K^{-} K^{+} \nu_{\tau}$
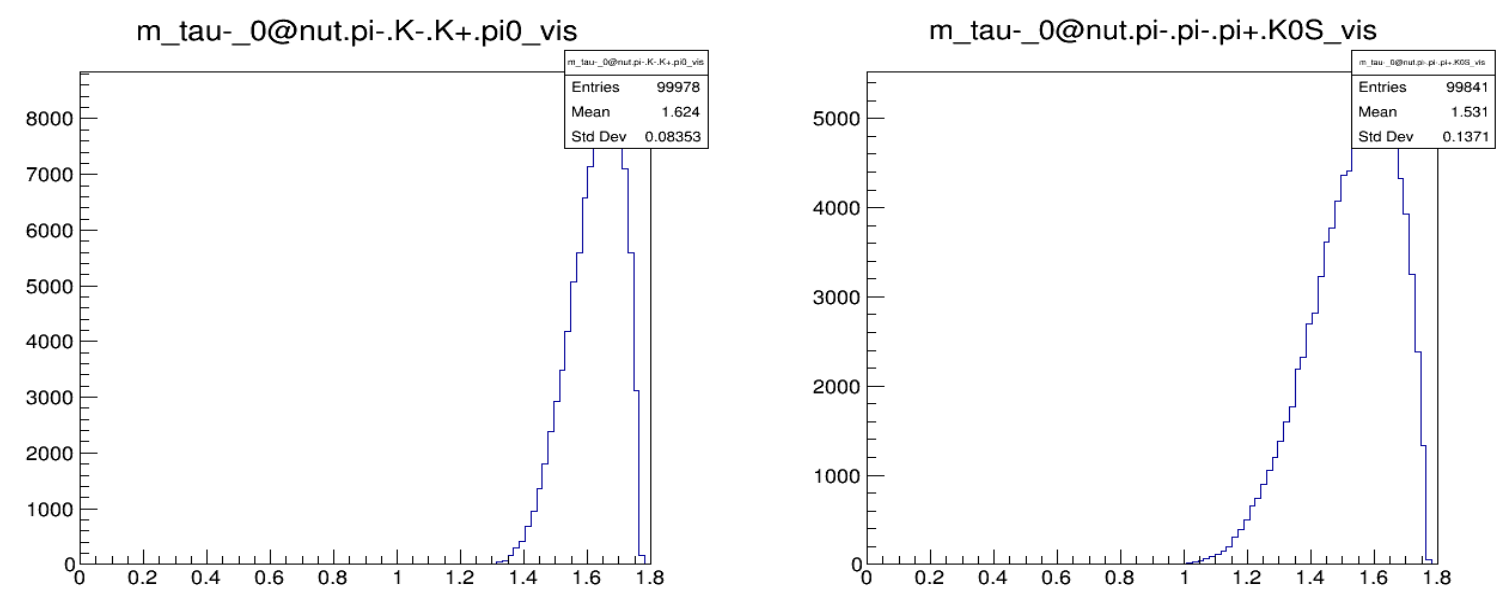

Figure 7.59: $m_{v i s}$ for $\tau^{-} \rightarrow$ Figure 7.60: $m_{v i s}$ for $\tau^{-} \rightarrow$ $\pi^{-} K^{-} K^{+} \pi^{0} \nu_{\tau}$ $\pi^{-} \pi^{+} \pi^{-} K_{S}^{0} \nu_{\tau}$
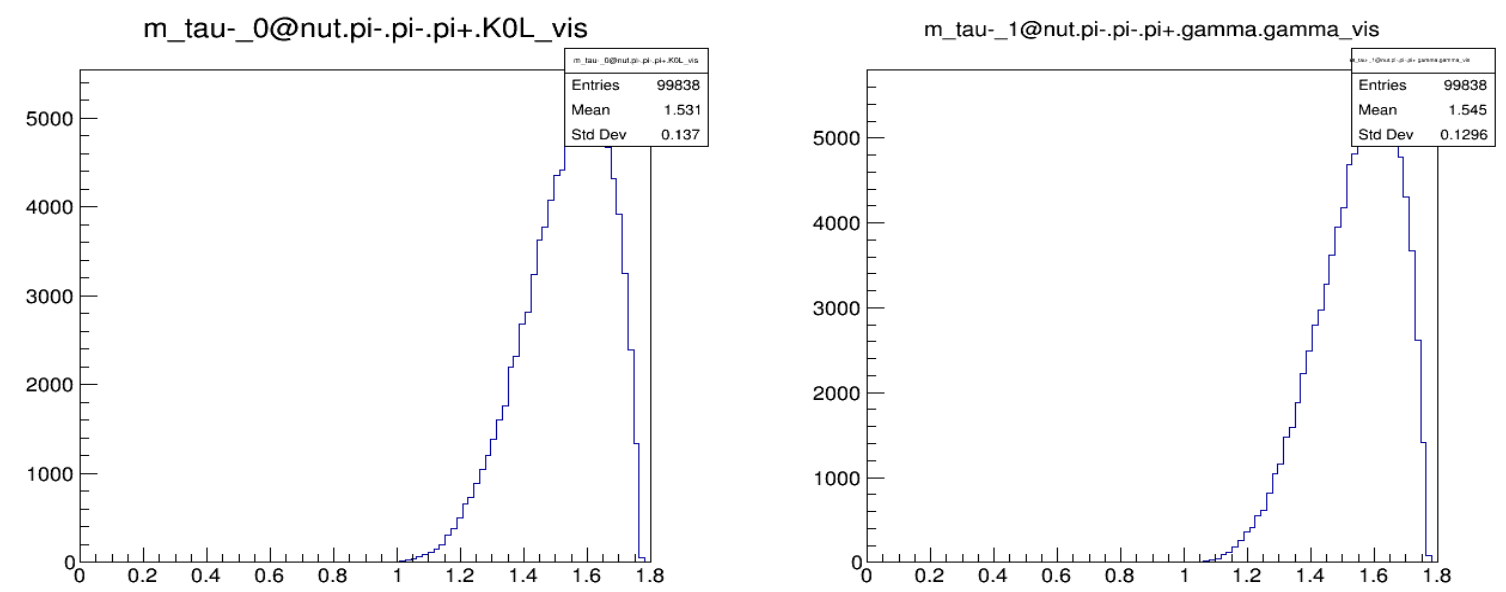

Figure 7.61: $m_{v i s}$ for $\tau^{-} \rightarrow$ $\pi^{-} \pi^{+} \pi^{-} K_{L}^{0} \nu_{\tau}$

Figure 7.62: $m_{v i s}$ for $\tau^{-} \rightarrow 2 \pi^{-} \pi^{+} \eta(\rightarrow$ $\gamma \gamma) \nu_{\tau}$ 
m tau-0@nut.pi-.pi-.pi+.pi0.pi0.pi0_vis

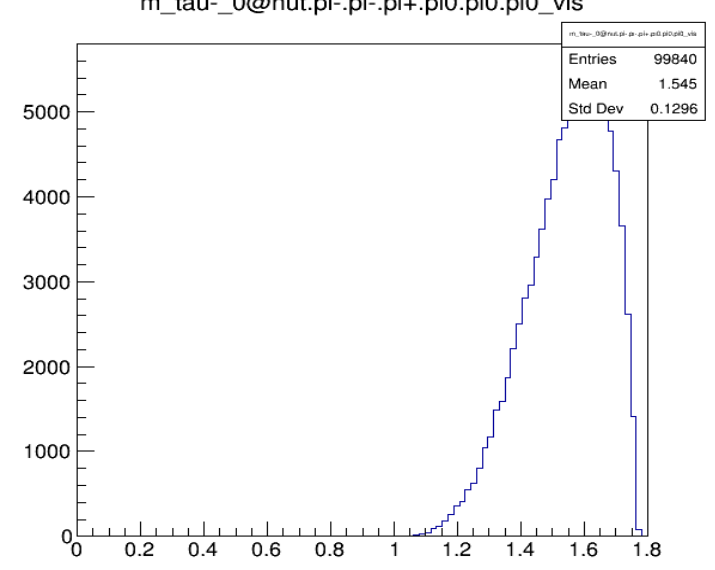

Figure 7.63: $m_{v i s}$ for $\tau^{-} \rightarrow 2 \pi^{-} \pi^{+} \eta(\rightarrow$ $\left.\pi^{0} \pi^{0} \pi^{0}\right) \nu_{\tau}$

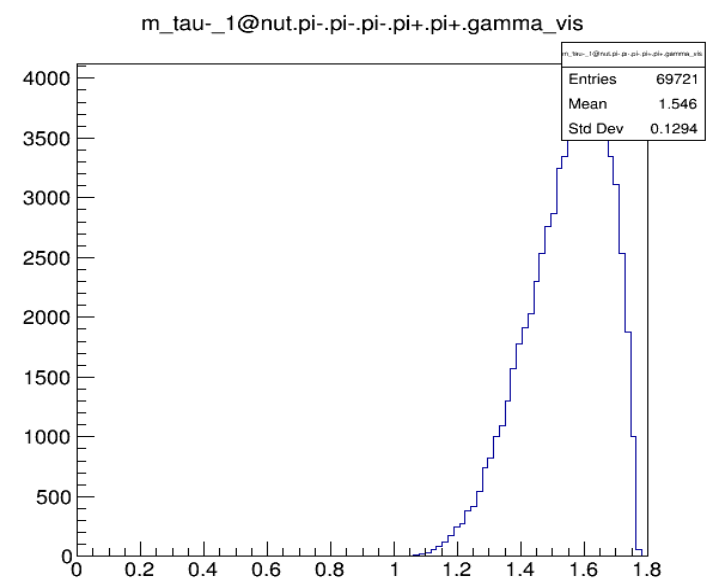

Figure 7.65: $m_{v i s}$ for $\tau^{-} \rightarrow 2 \pi^{-} \pi^{+} \eta(\rightarrow$ $\left.\pi^{+} \pi^{-} \gamma\right) \nu_{\tau}$

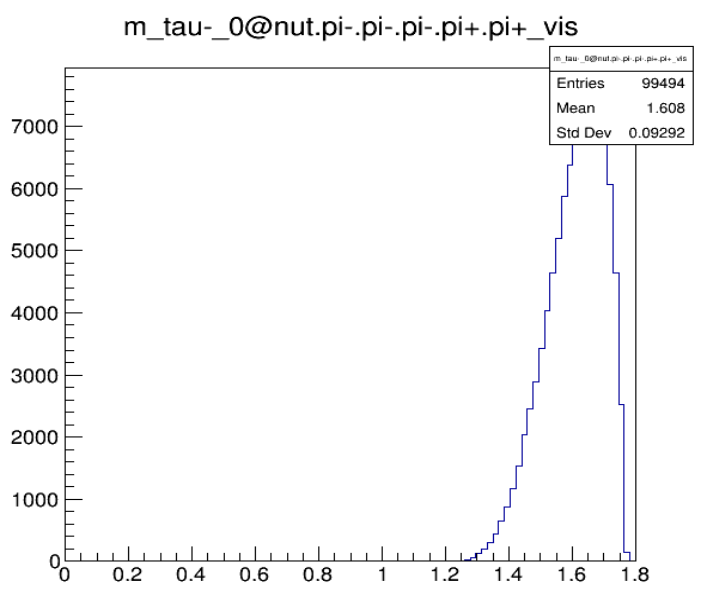

Figure 7.67: $m_{v i s}$ for $\tau^{-} \rightarrow 2 \pi^{-} \pi^{+} \omega(\rightarrow$ $\left.\pi^{+} \pi^{-}\right) \nu_{\tau}$ m tau-0@nut.pi-.pi-.pi-.pi+.pi+.pi0 vis

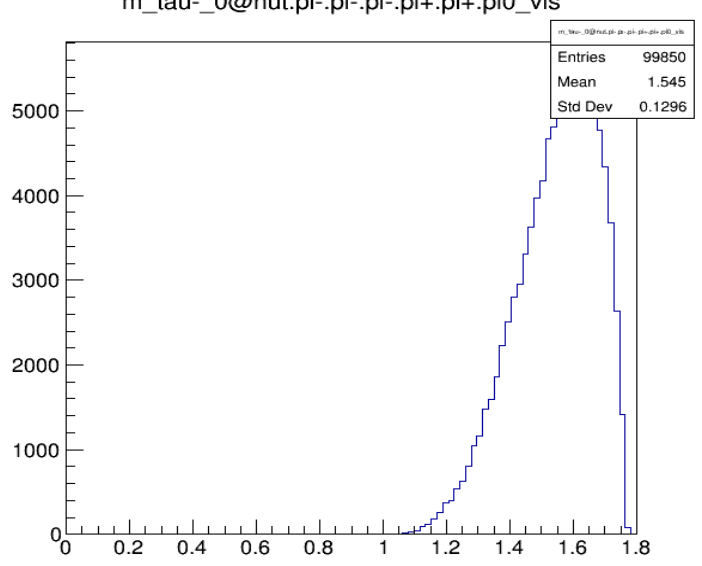

Figure 7.64: $m_{v i s}$ for $\tau^{-} \rightarrow 2 \pi^{-} \pi^{+} \eta(\rightarrow$ $\left.\pi^{+} \pi^{-} \pi^{0}\right) \nu_{\tau}$

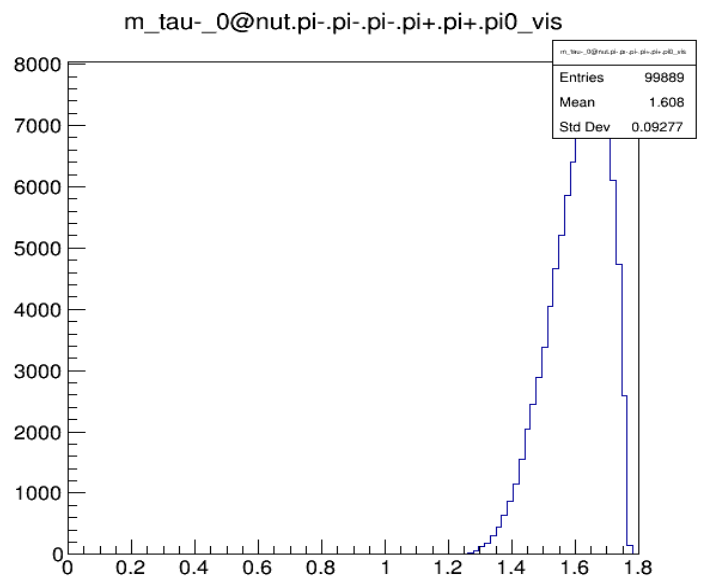

Figure 7.66: $m_{v i s}$ for $\tau^{-} \rightarrow 2 \pi^{-} \pi^{+} \omega(\rightarrow$ $\left.\pi^{+} \pi^{-} \pi^{0}\right) \nu_{\tau}$

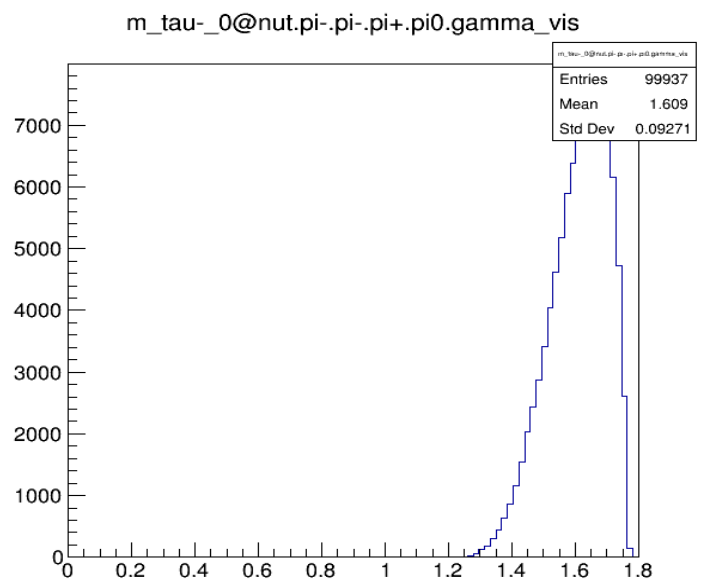

Figure 7.68: $m_{v i s}$ for $\tau^{-} \rightarrow 2 \pi^{-} \pi^{+} \omega(\rightarrow$ $\left.\pi^{0} \gamma\right) \nu_{\tau}$ 


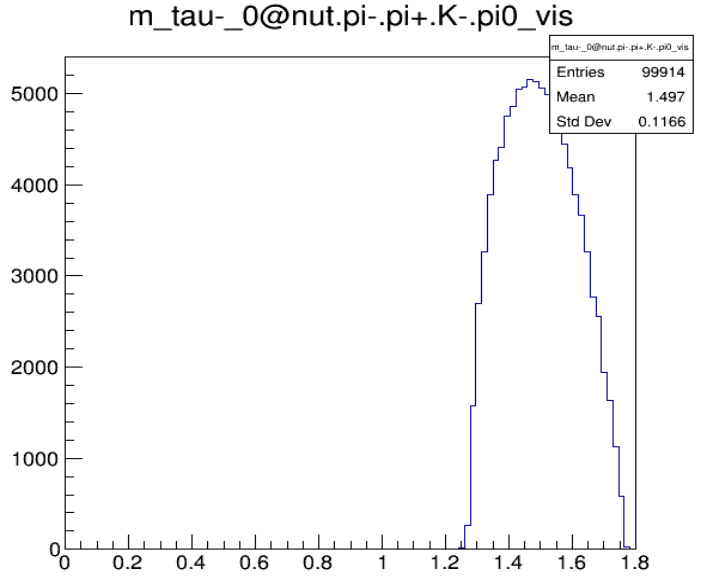

Figure 7.69: $m_{v i s}$ for $\tau^{-} \rightarrow K^{-} \omega(\rightarrow$ $\left.\pi^{+} \pi^{-} \pi^{0}\right) \nu_{\tau}$

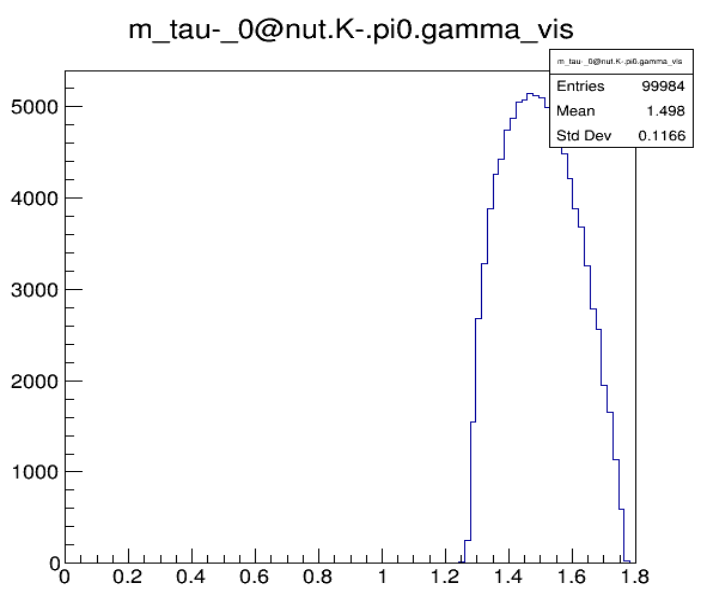

Figure 7.71: $m_{v i s}$ for $\tau^{-} \rightarrow K^{-} \omega(\rightarrow$ $\left.\pi^{0} \gamma\right) \nu_{\tau}$

m_tau-_0@nut.K-.KOS.KOL_vis

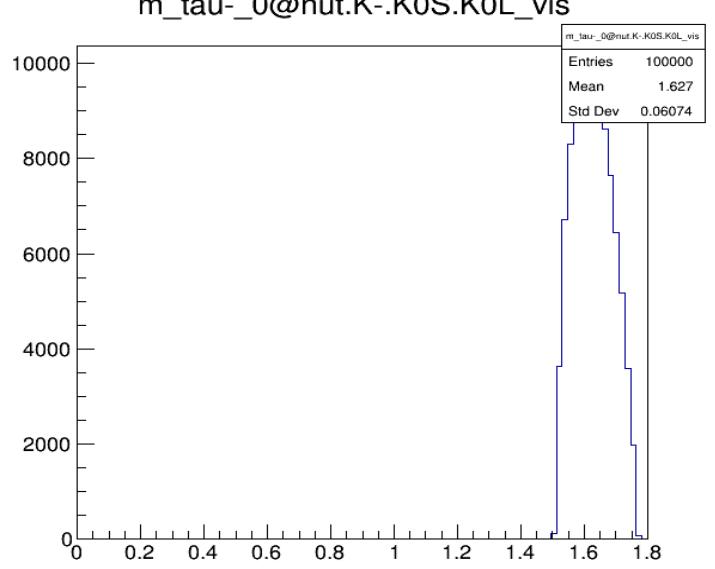

Figure 7.73: $m_{v i s}$ for $\tau^{-} \rightarrow K^{-} \phi(\rightarrow$ $\left.K_{S}^{0} K_{L}^{0}\right) \nu_{\tau}$
m_tau-_0@nut.pi-.pi+.K-_vis

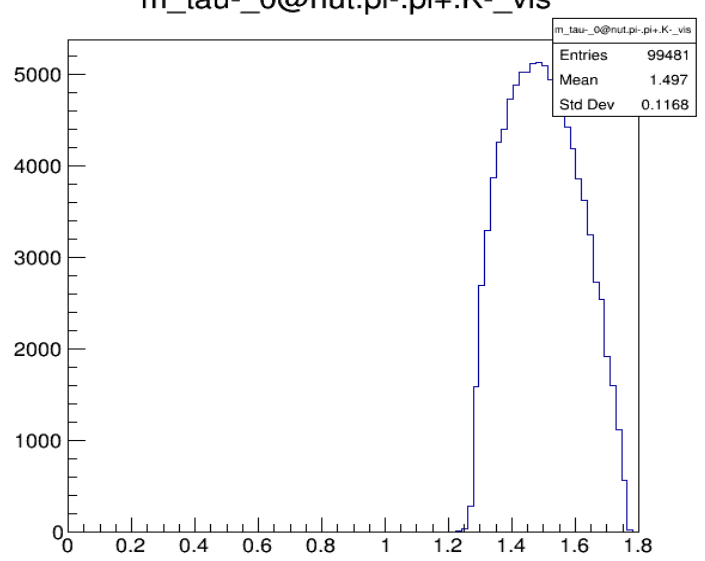

Figure 7.70: $m_{v i s}$ for $\tau^{-} \rightarrow K^{-} \omega(\rightarrow$ $\left.\pi^{+} \pi^{-}\right) \nu_{\tau}$

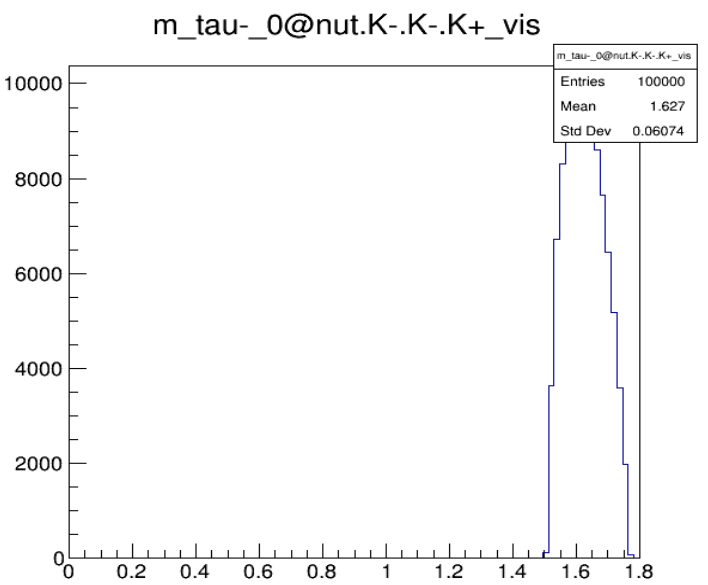

Figure 7.72: $m_{v i s}$ for $\tau^{-} \rightarrow K^{-} \phi(\rightarrow$ $\left.K^{+} K^{-}\right) \nu_{\tau}$

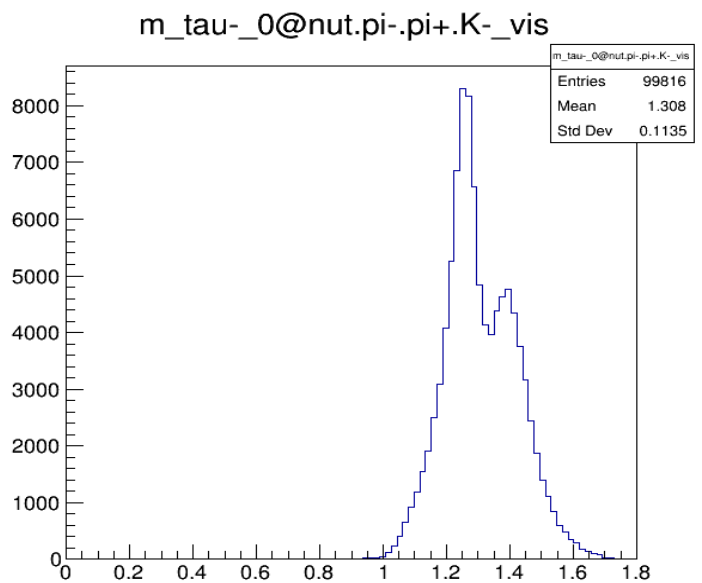

Figure 7.74: $m_{v i s}$ for $\tau^{-} \rightarrow K^{-} \pi^{-} \pi^{+} \nu_{\tau}$ 
m tau-0@nut.pi-.pi+.K-.pi0_vis

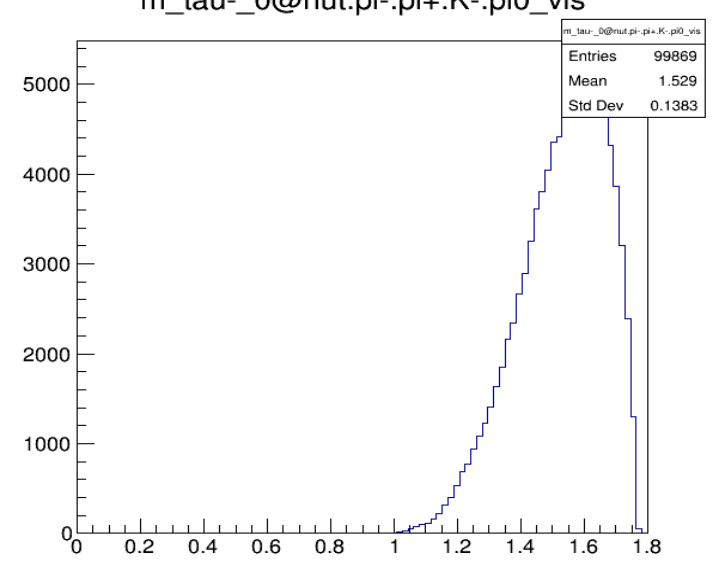

Figure 7.75: $m_{\text {vis }}$ for $\tau^{-} \rightarrow$ $K^{-} \pi^{-} \pi^{+} \pi^{0} \nu_{\tau}$

m_tau-_0@nut.pi-.pi-.pi+.pio.pio_vis

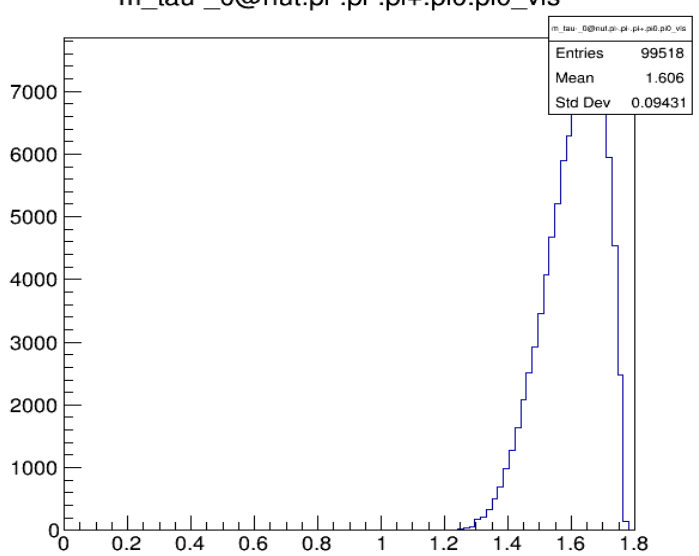

Figure 7.77: $m_{v i s}$ for $\tau^{-} \rightarrow \pi^{-} 2 \pi^{0} \omega(\rightarrow$ $\left.\pi^{+} \pi^{-}\right) \nu_{\tau}$

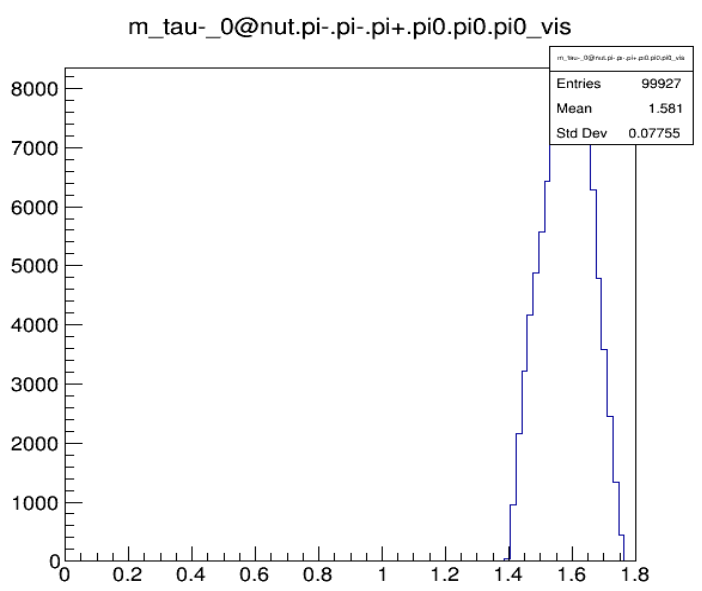

Figure 7.79: $m_{v i s}$ for $\tau^{-} \rightarrow 2 \pi^{-} \pi^{+} 3 \pi^{0} \nu_{\tau}$

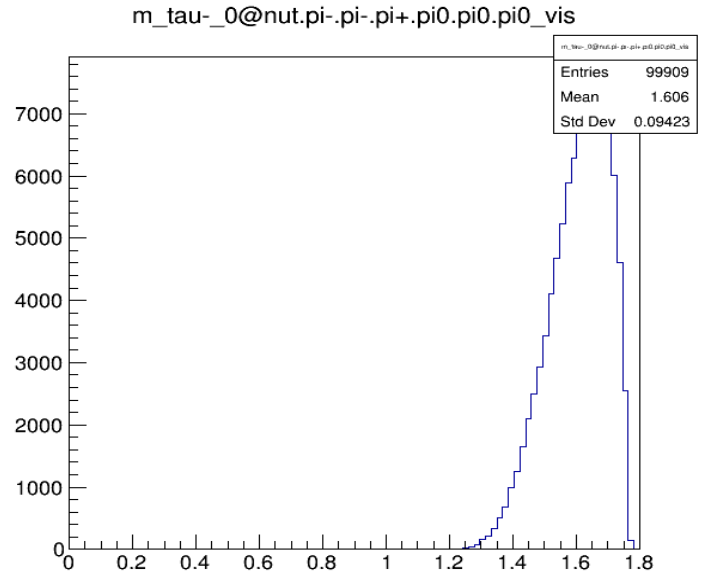

Figure 7.76: $m_{v i s}$ for $\tau^{-} \rightarrow \pi^{-} 2 \pi^{0} \omega(\rightarrow$ $\left.\pi^{+} \pi^{-} \pi^{0}\right) \nu_{\tau}$

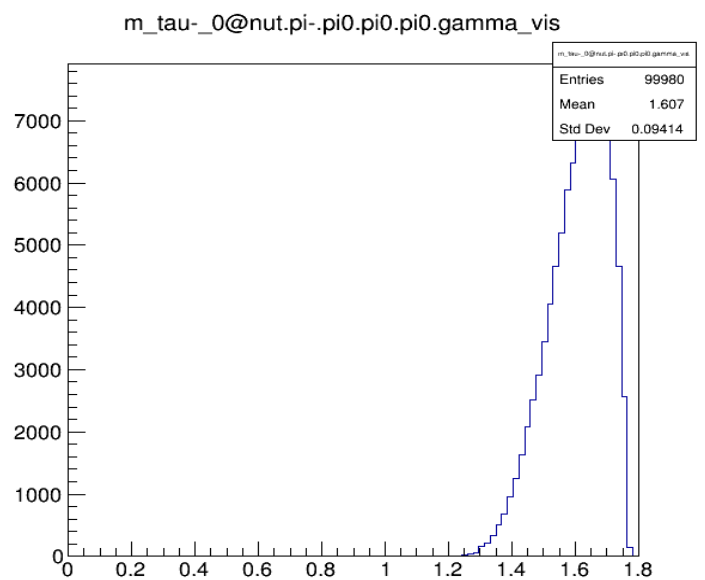

Figure 7.78: $m_{v i s}$ for $\tau^{-} \rightarrow \pi^{-} 2 \pi^{0} \omega(\rightarrow$ $\left.\pi^{0} \gamma\right) \nu_{\tau}$

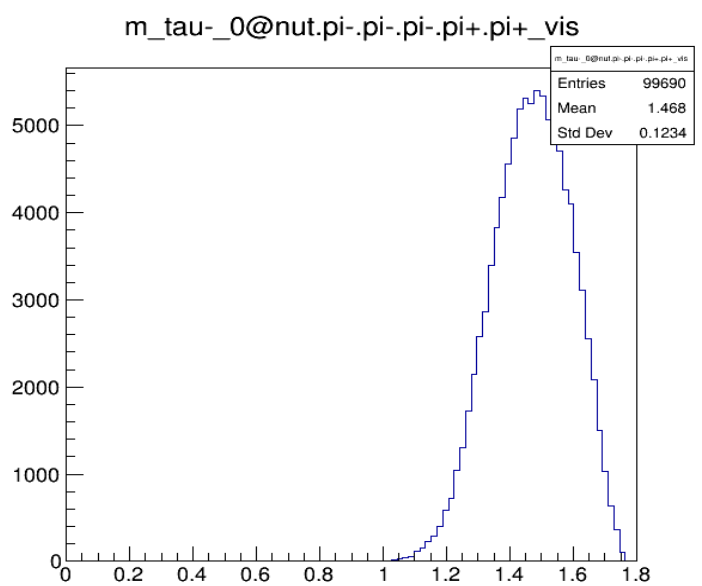

Figure 7.80: $m_{v i s}$ for $\tau^{-} \rightarrow 3 \pi^{-} 2 \pi^{+} \nu_{\tau}$ 
m tau-0@nut.pi-.pi-.pi+.pi+.K- vis

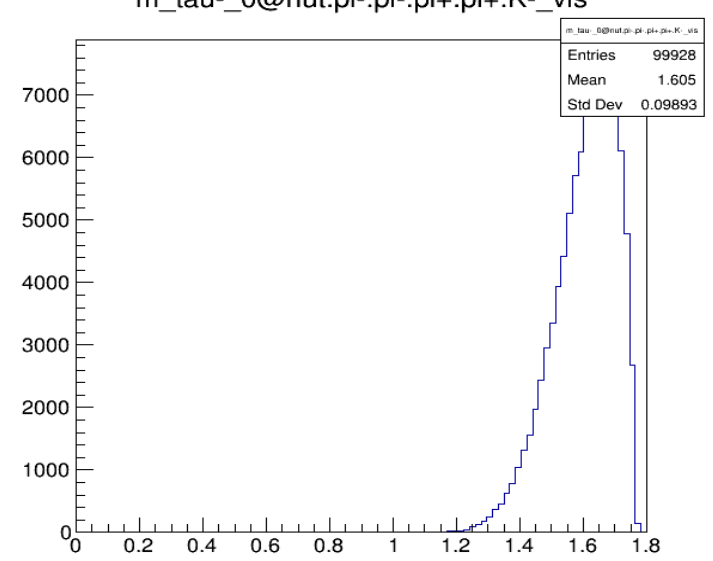

Figure 7.81: $m_{v i s}$ for $\tau^{-} \rightarrow$ $K^{-} 2 \pi^{-} 2 \pi^{+} \nu_{\tau}$

m_tau-_0@nut.pi-.pi-.pi+.pi+.K-.pi0_vis

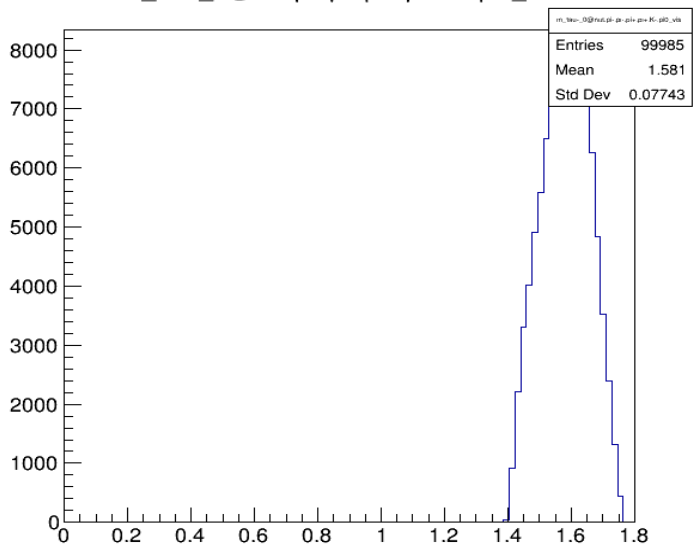

Figure 7.83: $m_{v i s}$ for $\tau^{-} \rightarrow$ $K^{-} 2 \pi^{-} 2 \pi^{+} \pi^{0} \nu_{\tau}$

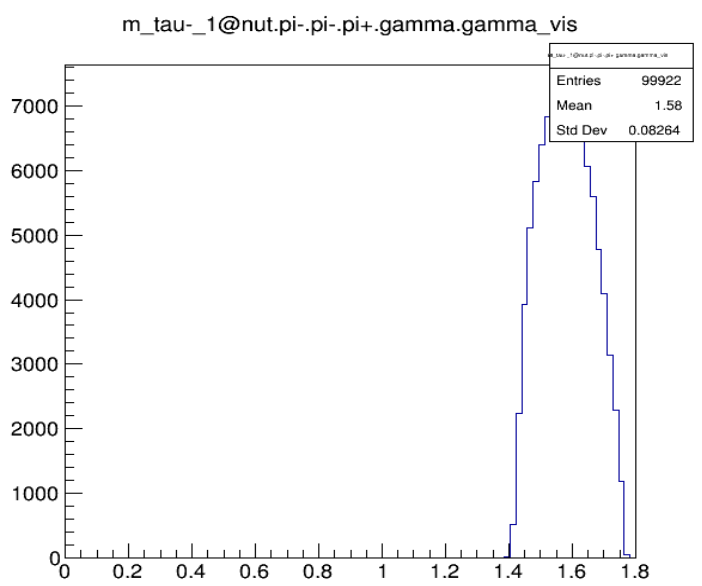

Figure 7.85: $m_{v i s}$ for $\tau^{-} \rightarrow \pi^{-} f_{1}(\rightarrow$ $\left.\pi^{-} \pi^{+} \eta\right) \nu_{\tau}$, where $\eta \rightarrow \gamma \gamma$ m tau-0@nut.pi-.pi-.pi-.pi+.pi+.pi0 vis

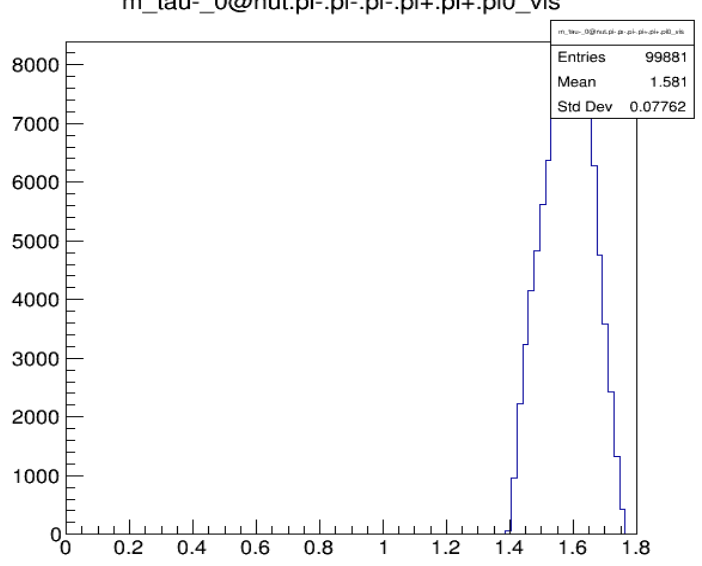

Figure 7.82: $m_{v i s}$ for $\tau^{-} \rightarrow 3 \pi^{-} 2 \pi^{+} \pi^{0} \nu_{\tau}$ m_tau-_0@nut.pi-.pi-.pi-.pi+.pi+_vis

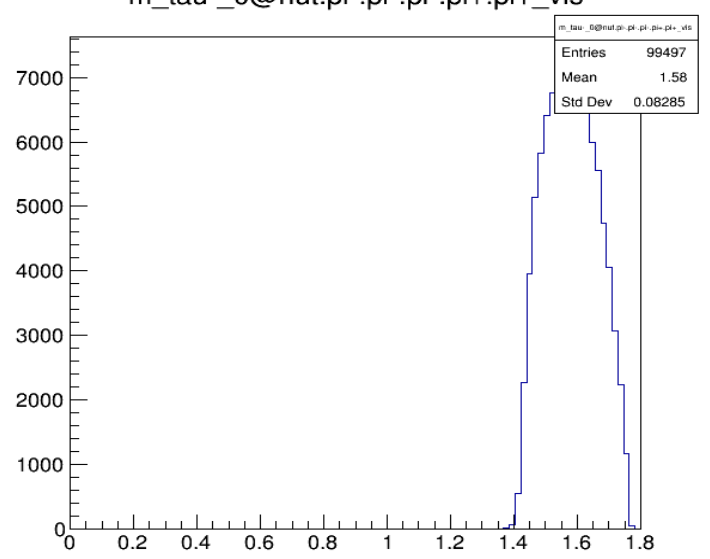

Figure 7.84: $m_{v i s}$ for $\tau^{-} \rightarrow \pi^{-} f_{1}(\rightarrow$ $\left.2 \pi^{-} 2 \pi^{+}\right) \nu_{\tau}$

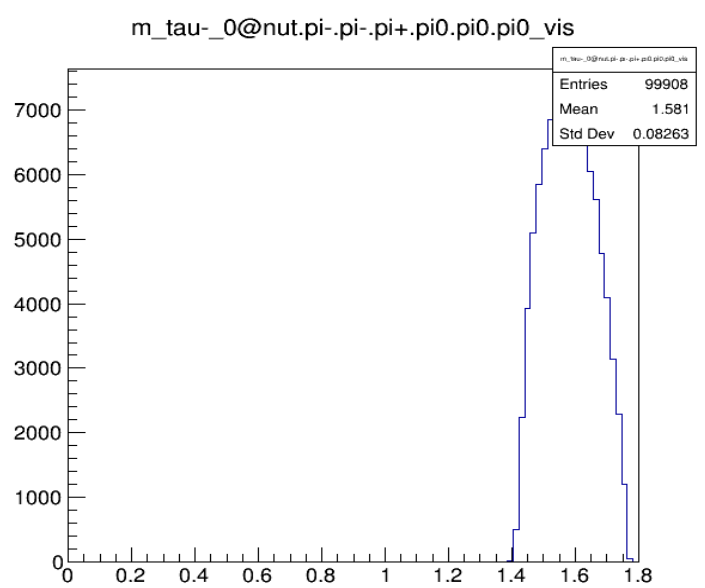

Figure 7.86: $m_{v i s}$ for $\tau^{-} \rightarrow \pi^{-} f_{1}(\rightarrow$ $\left.\pi^{-} \pi^{+} \eta\right) \nu_{\tau}$, where $\eta \rightarrow 3 \pi^{0}$ 
m tau-0@nut.pi-.pi-.pi-.pi+.pi+.pi0 vis

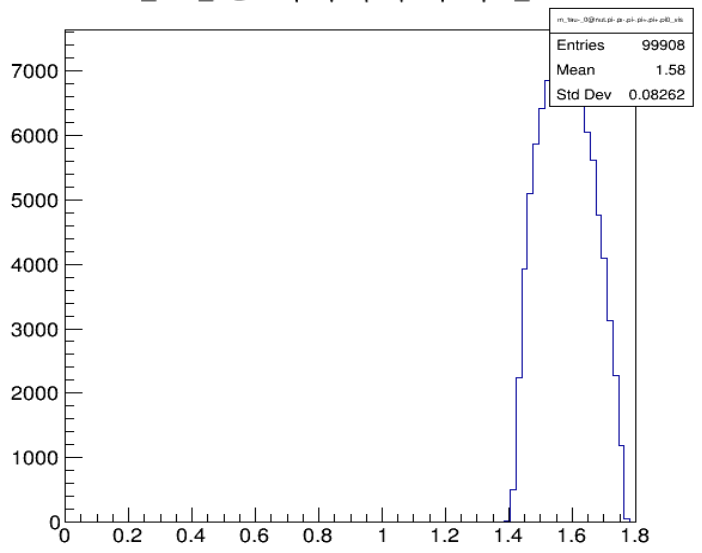

Figure 7.87: $m_{v i s}$ for $\tau^{-} \rightarrow \pi^{-} f_{1}(\rightarrow$ $\left.\pi^{-} \pi^{+} \eta\right) \nu_{\tau}$, where $\eta \rightarrow \pi^{-} \pi^{+} \pi^{0}$

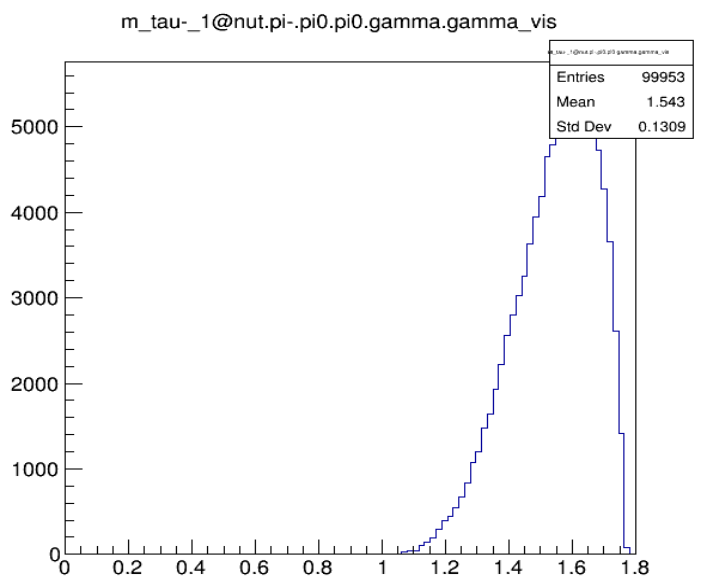

Figure 7.89: $m_{v i s}$ for $\tau^{-} \rightarrow \pi^{-} 2 \pi^{0} \eta \nu_{\tau}$, where $\eta \rightarrow \gamma \gamma$

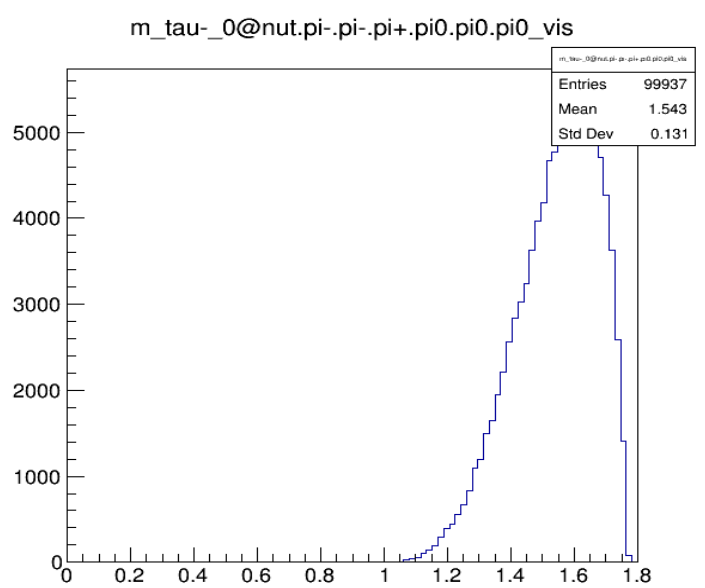

Figure 7.91: $m_{v i s}$ for $\tau^{-} \rightarrow \pi^{-} 2 \pi^{0} \eta \nu_{\tau}$, where $\eta \rightarrow \pi^{-} \pi^{+} \pi^{0}$

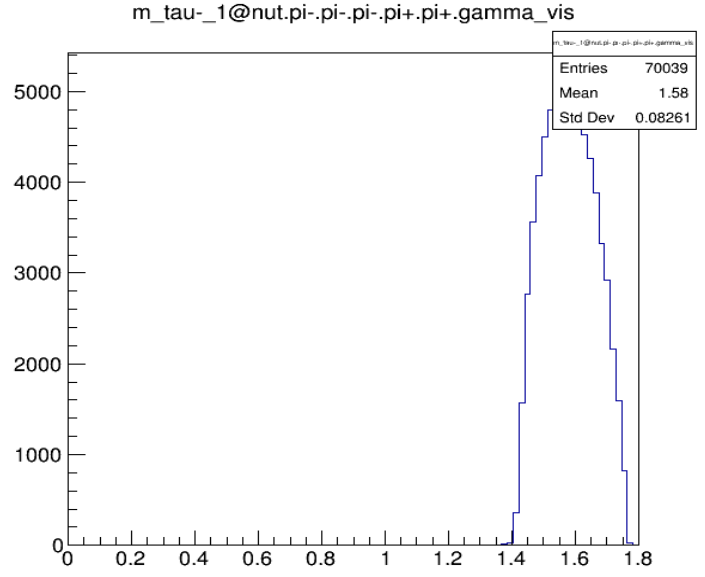

Figure 7.88: $m_{v i s}$ for $\tau^{-} \rightarrow \pi^{-} f_{1}(\rightarrow$ $\left.\pi^{-} \pi^{+} \eta\right) \nu_{\tau}$, where $\eta \rightarrow \pi^{-} \pi^{+} \gamma$

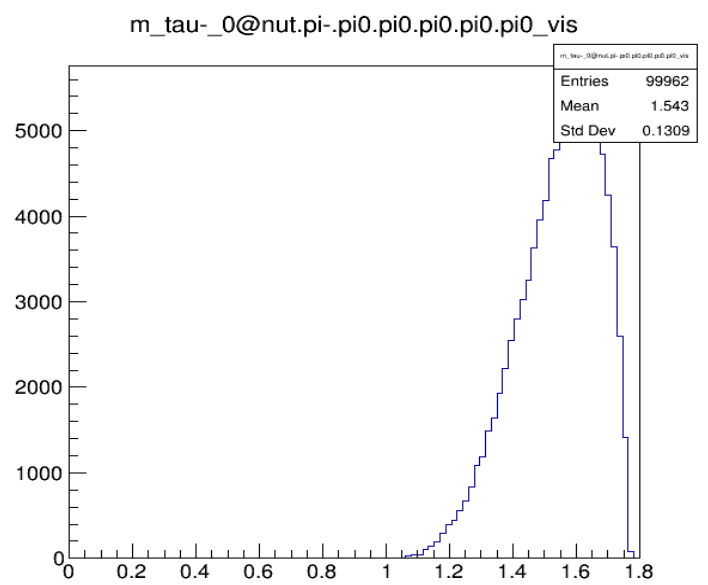

Figure 7.90: $m_{v i s}$ for $\tau^{-} \rightarrow \pi^{-} 2 \pi^{0} \eta \nu_{\tau}$, where $\eta \rightarrow 3 \pi^{0}$

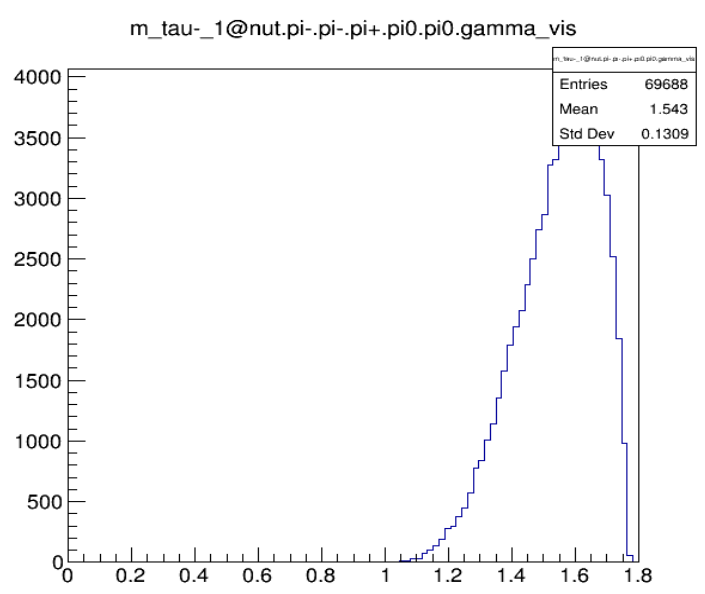

Figure 7.92: $m_{v i s}$ for $\tau^{-} \rightarrow \pi^{-} 2 \pi^{0} \eta \nu_{\tau}$, where $\eta \rightarrow \pi^{-} \pi^{+} \gamma$ 
CHAPTER VIII

\section{SEARCHES FOR LEPTON-FLAVOR-VIOLATING DECAYS OF THE HIGGS BOSON WITH THE ATLAS EXPERIMENT}

\subsection{Introduction}

Although indirect theoretical predictions on the LFV decays of the Higgs boson are $\mathcal{B}(H \rightarrow \ell \tau) \simeq 10 \%$ [22, 23], direct searches at the LHC impose much stricter limits. Using Run 1 data collected at the centre-of-mass energy $\sqrt{s}=8 \mathrm{TeV}$ corresponding to an integrated luminosity of $20.3 \mathrm{fb}^{-1}$, ATLAS searches [74] put a limit of $1.43 \%$ (1.04\%) on the $H \rightarrow \mu \tau(H \rightarrow e \tau)$ branching fraction with a $95 \%$ confidence level (CL). With a similar dataset at Run 1, the CMS collaboration has observed a $2.4 \sigma$ excess of events in their search for the decay $H \rightarrow \mu \tau$ [75]. Their best fit value for $\operatorname{BR}(H \rightarrow \mu \tau)$ was found to be $(0.9 \pm 0.4) \%$, and their observed limit was $\mathrm{BR}(H \rightarrow \mu \tau)<1.57 \%$. The observed $95 \% \mathrm{CL}$ limit on $\mathrm{BR}(H \rightarrow e \tau)$ is $0.69 \%$. The CMS Collaboration has recently provided 95\% CL upper limits on these branching ratios of $0.61 \%$ and $0.25 \%$, respectively, using data collected at $\sqrt{s}=13 \mathrm{TeV}$, with an integrated luminosity of $35.9 \mathrm{fb}^{-1}[76]$.

In this thesis, searches for LFV decays $H \rightarrow \mu \tau$ and $H \rightarrow e \tau$ of the Higgs boson at the LHC are presented. Studies are based on data recorded with the ATLAS detector 

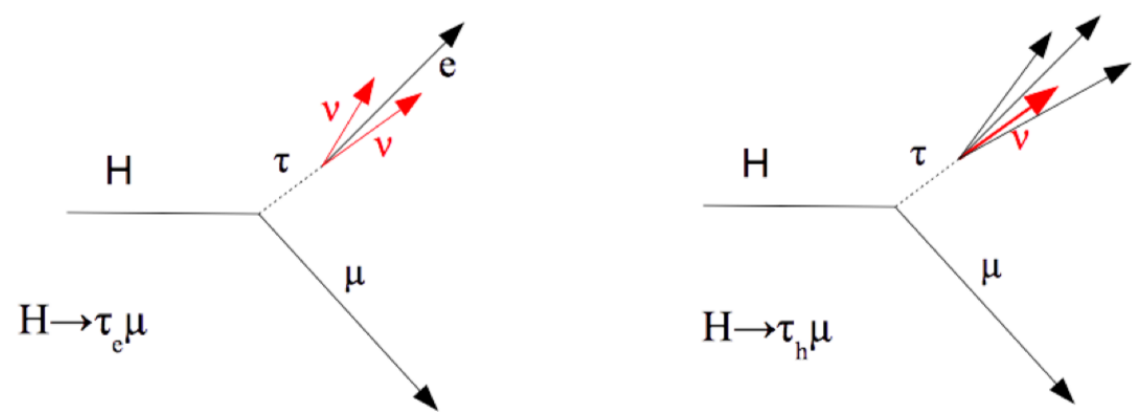

Figure 8.1: Final states in search for LFV decays $H \rightarrow \mu \tau$.

in 2015 and 2016 from $p p$ collision at $\sqrt{s}=13 \mathrm{TeV}$. The dataset corresponds to an integrated luminosity of $36.1 \mathrm{fb}^{-1}$.

The searches presented here involve both leptonic and hadronic decays of $\tau$ leptons, denoted $\tau_{\text {lep }}$ and $\tau_{\text {had }}$ respectively, as illustrated for $H \rightarrow \mu \tau$ in Figure 8.1. The dilepton final state $\ell \tau_{\ell^{\prime}}$ only considers pairs of different-flavour leptons. Sameflavour lepton pairs are not considered due to the large lepton pair-production from decays of the $Z$ boson known as the Drell-Yan background. Thus, two channels are considered for each of the two searches: $e \tau_{\mu}$ and $e \tau_{\text {had }}$ for the $H \rightarrow e \tau$ search, $\mu \tau_{e}$ and $\mu \tau_{\text {had }}$ for the $H \rightarrow \mu \tau$ search.

Potential overlap between the $H \rightarrow e \tau$ and $H \rightarrow \mu \tau$ searches are designed to be negligible by construction. The two searches are very similar, allowing the analyses to be almost identical. There are however some key differences in the composition of the backgrounds. $Z / \gamma^{*} \rightarrow \tau \tau$ is one of the main backgrounds for both, followed by top-quark production in the $\ell \tau_{\ell^{\prime}}$ channel and by the fake- $\tau$ backgrounds in the $\ell \tau_{\text {had }}$ channel. The analysis was thoroughly reviewed by the collaboration [77], before proceeding to the unblinding of the phase space of the signal region with highest signal expectation. The main results are based on a Multi-Variate Analysis (MVA). A cut-based analysis (CBA) cross-check is also performed.

Data and Monte Carlo samples are discussed in Section 8.2, followed by a discussion on object reconstruction in Section 8.3. Event selection is described in Section 8.4 
and background modelling is discussed in Section 8.5. More details of the analysis, particularly the background modelling are presented for the $\ell \tau_{\text {had }}$ channels, as it one of my primary contributions. Systematic uncertainities and pre-fit results appear in Section 8.6. Statistical fit, final results and conclusions are presented in Sections 8.7, 8.8 and 8.9 respectively.

\subsection{Data Samples and Monte Carlo Simulation}

The data used in this analysis were recorded during Run 2 in proton-proton collisions at the LHC where proton bunches collided every 25 ns at $\sqrt{s}=13 \mathrm{TeV}$. A combination of several triggers for single leptons and hadronically decaying $\tau$-lepton were used to record the data for this analysis, depending on the analysis channel. After data quality requirements, the sample used for this measurement consist of 3.2 $\mathrm{fb}^{-1}$ of data recorded in 2015, with an average of 14 interactions per bunch crossing, and $32.9 \mathrm{fb}^{-1}$ recorded in 2016, with an average of 25 interactions per bunch crossing ${ }^{1}$.

\subsubsection{Simulation samples}

Samples of Monte Carlo (MC) simulated events are used to optimize the event selection, and to model the signal and several of the background processes. The samples were produced with the ATLAS simulation infrastructure [78] using the full detector simulation performed by the GEANT4 [79] toolkit. The Higgs boson mass was set to $m_{H}=125 \mathrm{GeV}[80]$. The four leading Higgs boson production mechanisms are considered: the gluon-gluon fusion ( $\mathrm{ggF})$, vector-boson fusion (VBF) and two associated production modes $(W H, Z H)$, while the others having negligible contributions are

\footnotetext{
${ }^{1}$ The good run lists used are data15_13TeV.periodAllYear_DetStatus-v79-repro20-02_ DQDefects-00-02-02_PHYS_StandardGRL_All_Good_25ns.xml for 2015 data and data16_13TeV. periodAllYear_DetStatus-v88-pro20-21_DQDefects-00-02-04_PHYS_StandardGRL_AlI_Good_ 25ns for 2016 data.
} 
ignored. The cross-sections of all Higgs boson production processes were normalized to the SM predictions [81]. The LFV Higgs boson decays as well as the $H \rightarrow \tau \tau$ and $H \rightarrow W W$ background decays were modelled with Pythia 8 [82]. Other background processes involve electroweak production of $W / Z$ bosons via VBF, Drell-Yan production of $W / Z$ in association with jet(s) as well as diboson, single top-quark and top-quark pair $(t \bar{t})$ production. The MC generators used for the SM $H \rightarrow \tau \tau$ crosssection measurement [83] were also employed. The generators and parton shower models used to simulate different processes are summarized in Table 8.1. 


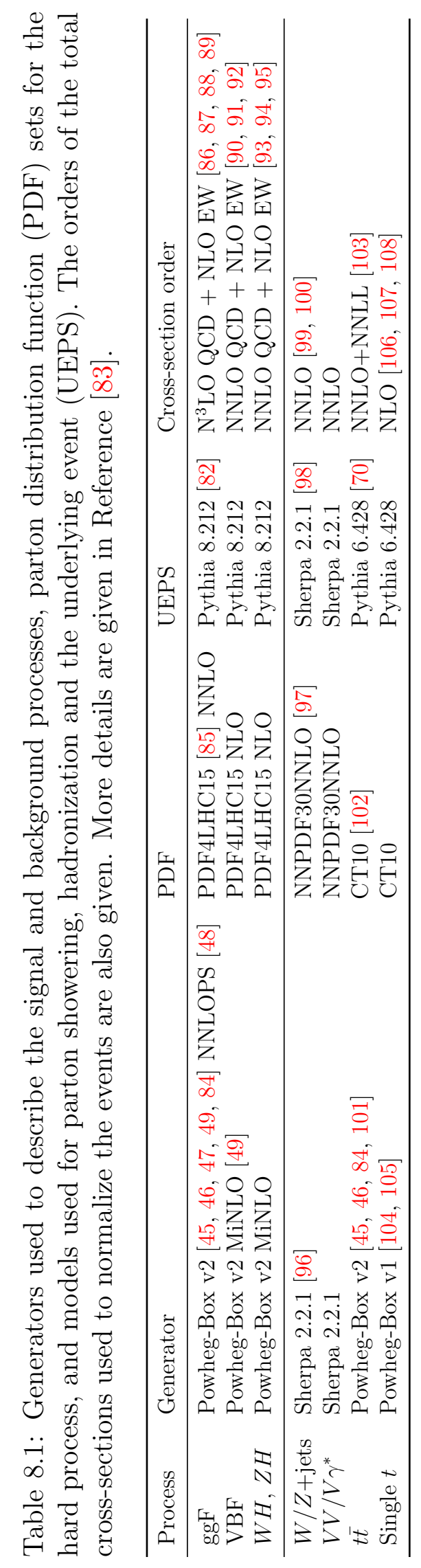




\subsection{Object Reconstruction}

The correct identification of $H \rightarrow \ell \tau$ events requires reconstruction of most physics objects (electrons, muons, $\tau$-leptons, jets) and the missing transverse momentum $\vec{E}_{\mathrm{T}}^{\mathrm{miss}}$. In order to remove ambiguities in the object reconstruction, an overlap removal (OLR) procedure is applied between nearby objects based on $\Delta R$ and/or shared reconstructed tracks [83].

\subsubsection{Electrons}

Electron reconstruction begins with clustered energy deposits in the electromagnetic calorimeter that are matched to tracks in the inner detector. Electron candidates are required to pass a "loose" likelihood-based identification selection point, have $p_{\mathrm{T}}>15 \mathrm{GeV}$ and to be in the fiducial volume of the detector, $|\eta|<2.47$. The transition region between the barrel and end-cap calorimeters $(1.37<|\eta|<1.52)$ is excluded. After the overlap removal is performed, a medium identification and gradient isolation criteria are used for the electrons in this analysis, corresponding to an efficiency of $87 \%$ at $p_{\mathrm{T}}=20 \mathrm{GeV}[109]$.

\subsubsection{Muons}

Muon candidates are identified by tracks reconstructed in the muon spectrometer and matched to tracks reconstructed in the inner detector. Loose identification [110], $p_{\mathrm{T}}>10 \mathrm{GeV}$ and $|\eta|<2.5$ requirements are applied. Medium identification (efficiency of $96.1 \%$ for muons with $p_{\mathrm{T}}>20 \mathrm{GeV}$ ) [110] is imposed for the baseline muon selection. Similarly to electron definition, a loose muon identification is used for the overlap removal, but it is later tightened to medium identification and gradient isolation in the analysis selection. The gradient working point is used, featuring an

efficiency of $90 \%$ (99\%) obtained for leptons with $p_{\mathrm{T}}>25 \mathrm{GeV}(60 \mathrm{GeV})$ originating 
from the $Z \rightarrow \ell \ell$ process $[109,110]$.

\subsubsection{Jets}

Jets are reconstructed using the anti- $k_{t}$ algorithm [111] as implemented by the FastJet [112] package. The algorithm is applied to topological clusters of calorimeter cells [113] with a radius parameter $R=0.4$. Only jets with $p_{\mathrm{T}}>20 \mathrm{GeV}$ and $|\eta|<4.5$ are considered. Jets from other $p p$ interactions in the same and neighbouring bunch crossings (pile-up) are suppressed using jet vertex tagger (JVT) algorithms [114, 115]. Jets containing $b$-hadrons ( $b$-jets) are identified by the MV2c20 algorithm $[116,117]$ in the central region $(|\eta|<2.4)$. A working point corresponding to $85 \%$ average efficiency determined for $b$-jets in $t \bar{t}$ simulated events is chosen. Rejection factors are 2.8 and 28 against $c$-jets and light-flavour jets respectively.

\subsection{4 $\tau$ decays}

Leptonic $\tau$-decays are reconstructed as electrons or muons. The reconstruction of the object formed by the visible products of the $\tau_{\text {had }}$ decay $\left(\tau_{\text {had-vis }}\right)$ begins from jets reconstructed by the anti- $k_{t}$ jet algorithm with a radius parameter $R=0.4$. Information from the inner detector tracks associated with the energy deposits in the calorimeter is incorporated in the reconstruction. Only $\tau_{\text {had-vis }}$ candidates with $p_{\mathrm{T}}>20 \mathrm{GeV}$ and $|\eta|<2.5$ are considered. ${ }^{2}$ One or three associated tracks with an absolute total charge $|q|=1$ are required. An identification algorithm [118, 119] based on boosted decision trees (BDT) $[120,121,122]$ is used to reject $\tau_{\text {had-vis }}$ candidates arising from misidentification of jets or from decays of hadrons with $b$ - or $c$-quark content. Unless otherwise indicated, a tight identification (ID) working point is used for the $\tau_{\text {had-vis }}$, corresponding to an efficiency of $60 \%$ (45\%) for 1-prong (3-prong) candidates. Jets corresponding to identified $\tau_{\text {had-vis }}$ candidates are removed from the

\footnotetext{
${ }^{2}$ The transition region in $\eta$ is excluded, similar to electrons.
} 
jet collection. The $\tau_{\text {had-vis }}$ candidates with one track overlapping with an electron candidate with high ID score, as determined by a multivariate (MVA) approach, are rejected.

\subsubsection{Missing transverse energy}

The missing transverse energy $\left(\vec{E}_{\mathrm{T}}^{\text {miss }}\right)$ definition used in this analysis is an objectbased definition [123]. It is computed using the fully calibrated and reconstructed

physics objects as described above. The SoftTerm of the $\vec{E}_{\mathrm{T}}^{\text {miss }}$ is computed using the TrackSoftTerm (TST) algorithm as it is the default for Run 2 analysis.

\subsubsection{Overlap removal}

Geometric overlap between objects passing the above selection creates ambiguity in the identity of the objects. Thus an overlap removal is applied between the objects whose $\Delta R$ (defined as: $\Delta R \equiv \sqrt{\Delta \phi^{2}+\Delta \eta^{2}}$ ) is less than a certain threshold. When two objects do not match this requirement, the one that is kept is following this order: muons, electron, taus and jets. This strategy follows the general recommendations for overlap removal and the recommendations of the TauCP group.

The $\Delta R$ threshold is not the same for the different combinations and hence, is defined below:

- Jets within a $\Delta R=0.2$ cone of the leading $p_{\mathrm{T}} \tau_{\text {had }}$ are excluded.

- Jets within a $\Delta R=0.2$ cone of an electron or muon are excluded.

- $\tau_{\text {had }}$ within a $\Delta R=0.2$ cone of electrons or muons are excluded.

- Electrons within a $\Delta R=0.2$ cone of muons are excluded.

The muon definition for the overlap removal are chosen more loose than the object definitions given above. Here, muons are required to pass the "loose" identification 
and a minimum $p_{\mathrm{T}}$ of $2 \mathrm{GeV}$. The details of the removal of hadronic taus overlapping with electrons is described above in the tau object definition.

\subsection{7 $\ell \tau$ mass reconstruction}

A schematic description of the process $H \rightarrow \mu \tau$ is shown in Figure 8.2.

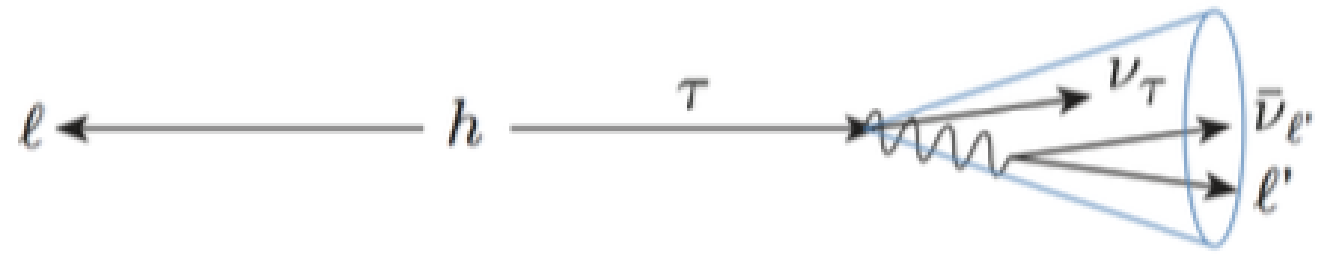

Figure 8.2: Schematic description of a signal process. In this example a leptonic decay of the $\tau$ is considered.

In the Higgs rest frame, the $\tau$ and the light lepton are produced back to back and the $\tau$ decay products are boosted along the same direction of the $\tau$.

The invariant mass of the Higgs boson reconstructed under the $H \rightarrow \ell \tau$ decay hypothesis exhibits the highest signal-to-background separation power and it helps to distinguish LFV signal from $H \rightarrow \tau \tau$ and $H \rightarrow W W$ backgrounds.

Two mass reconstruction methods are considered in the analysis:

- The so-called collinear approximation assumes that the direction of the $\tau$ is estimated from the direction of its visible decay products, and the momentum

of the $\tau$ is estimated starting from its visible decay product and the $\vec{E}_{\mathrm{T}}^{\mathrm{miss}}$ of the event:

$$
\begin{array}{r}
\ell \tau_{\ell^{\prime}}: \quad{\overrightarrow{p_{\mathrm{T}}}}^{\tau}={\overrightarrow{p_{\mathrm{T}}}}^{\ell^{\prime}}+{\overrightarrow{E_{\mathrm{T}}}}^{\text {miss }} \\
\ell \tau_{\text {had }}: \quad{\overrightarrow{p_{\mathrm{T}}}}^{\tau}={\overrightarrow{p_{\mathrm{T}}}}^{\tau_{\text {had-vis }}+{\overrightarrow{E_{\mathrm{T}}}}^{\text {miss }}}
\end{array}
$$


The collinear approximation allows to calculate the invariant mass of a system, called collinear mass $\left(m_{\text {coll }}\right)$. The rapidity $(y)$ and transverse mass $\left(m_{T}\right)$ of a particle are given by:

$$
y=\frac{1}{2} \ln \left(\frac{E+p_{z}}{E-p_{z}}\right), \quad m_{\mathrm{T}}=\sqrt{m^{2}+p_{\mathrm{T}}^{2}},
$$

Accordingly, the four-momentum, $p=\left(E, p_{x}, p_{y}, p_{z}\right)$ can be expressed as:

$$
p=\left(m_{\mathrm{T}} \cosh (y), p_{\mathrm{T}} \cos (\phi), p_{\mathrm{T}} \sin (\phi), m_{\mathrm{T}} \sinh (y)\right) .
$$

In the case of a mass-less particle $(m=0)$ or a particle with negligible mass, the four-momentum can be written as:

$$
p=p_{\mathrm{T}}(\cosh (\eta), \cos (\phi), \sin (\phi), \sinh (\eta)),
$$

where $\phi$ is the azimuthal angle and $\eta$ is the pseudo-rapidity.

Using the formula for the Lorentz invariant $s=p_{H}^{2}=\left(p_{\ell}+p_{\tau}\right)^{2}$ and Eq. 8.5, it is possible to calculate the invariant mass of the system as:

$$
m_{H}=\sqrt{2 p_{\mathrm{T}}^{\ell} p_{\mathrm{T}}^{\tau}(\cosh (\Delta \eta)-\cos (\Delta \phi))}
$$

where $\Delta \eta$ and $\Delta \phi$ are the difference in rapidity and azimuthal angle between the leading lepton and the sub-leading lepton, either a light lepton or a $\tau_{\text {had-vis }}$ for the $\ell \tau_{\ell^{\prime}}$ and $\ell \tau_{\text {had }}$ channels, respectively. The collinear approximation is finally used to close the kinematics. From Eqs. 8.5 and 8.6 the collinear mass can be 
defined as follows:

$$
\begin{aligned}
& \ell \tau_{\ell^{\prime}}: m_{\text {coll }}=\sqrt{2 p_{\mathrm{T}}^{\ell_{\text {ead }}}\left(p_{\mathrm{T}}^{\ell_{\text {sub-lead }}}+E_{\mathrm{T}}^{\text {miss }}\right)(\cosh (\Delta \eta)-\cos (\Delta \phi))} \\
& \ell \tau_{\text {had }}: m_{\text {coll }}=\sqrt{2 p_{\mathrm{T}}^{\ell}\left(p_{\mathrm{T}}^{\tau_{\text {had-vis }}}+E_{\mathrm{T}}^{\text {miss }}\right)(\cosh (\Delta \eta)-\cos (\Delta \phi))}
\end{aligned}
$$

- The Missing Mass Calculator (MMC) [124] is a reconstruction method firstly used for the mass reconstruction of the $H \rightarrow \tau^{+} \tau^{-}$decay. The method is adapted to LFV decays of the Higgs boson by assuming that all the source of missing momentum originates from the neutrinos in the single tau decay of the event, reducing the complexity of the method with respect to the $H \rightarrow \tau^{+} \tau^{-}$ decay.

The invariant mass is reconstructed using the missing mass solving an underconstrained system. The unknowns include the $x$-, $y$-, and $z$-components of the momentum carried by the neutrinos of the tau lepton in the event. The calculation uses the constraints from the measured $x$ - and $y$-components of the missing transverse momentum, and the visible masses of both the tau and light lepton candidates. A scan is performed over the two components of the missing transverse momentum vector and the yet undetermined variables. Each scan point is weighted by its probability according to the $\vec{E}_{\mathrm{T}}^{\text {miss }}$ resolution and the tau decay topologies. The estimator for the $\tau \ell$ mass is defined as the most probable value of the scan points. The estimator for the final discriminant, the mass of the two leptons system $m_{M M C}$, is then defined as the maximum of the histogram that has been filled with the weighted scan points.

The performance of MMC is highly correlated with the $\vec{E}_{\mathrm{T}}^{\mathrm{miss}}$ resolution. In order to compensate this effect, MMC is adjusted to allow for possible mismeasurements in $\vec{E}_{\mathrm{T}}^{\text {miss }}$ by increasing the dimensionality of the parameter space in which the scanning is performed to include the two components of the $\vec{E}_{\mathrm{T}}^{\text {miss }}$ 
resolution (for $E_{\mathrm{x}}^{\mathrm{miss}}$ and $E_{\mathrm{y}}^{\mathrm{miss}}$ ). Thus the event probability, accounts for the corresponding resolution functions for the $\vec{E}_{\mathrm{T}}^{\text {miss }}$. The MMC algorithm provides a solution for more than $99 \%$ of the $H \rightarrow \tau \ell$ events.

Based on the observed resolution of the simulated reconstructed signal events passing the event selection described in Section 8.4, the MMC mass is found to be optimally performant for $\ell \tau_{\ell^{\prime}}$ channel, while the the collinear mass is found be optimal for the $\ell \tau_{\text {had }}$ channel.

\subsubsection{BDT variable}

The analysis exploits the Boosted Decision Tree (BDT) algorithms [120, 121, 122] to enhance the signal separation from the background in the individual channels and categories. The parameters scanned over for the BDT are:

- Max depth controls the maximum depth on any given decision tree. Deeper networks are more sensitive but risk overtraining.

- Number of trees controls the number of boosts

- One effective way to slow down the learning in a gradient boosting model is to use a learning rate, also called shrinkage.

The definition of the BDT parameters for $\ell \tau_{\ell^{\prime}}$ and $\ell \tau_{\text {had }}$ channels are shown in Table 8.2 and 8.3 , respectively. The algorithm divides-up the space into signal-like and background-like regions and assigns a score to each event depending on which region it falls into. Boosted decision trees are trained on samples of known composition and tested on statistically independent samples.

All Higgs production modes are considered in the definition of the signal-like events. Similarly, all the background processes, including fake leptons, are considered in the definition of the background-like events. The strategy involved in the TrainingTest part is about $80 \%$ and $20 \%$ of the whole accepted events respectively. The 
events have been randomly split based on their event number. In this analysis, crossvalidation is performed splitting the data in five sets. Each of the five trainings is performed with $80 \%$ of the accepted events and it is tested with the remaining $20 \%$. Each of the testing samples is statistically independent from the others, as well as independent from the events used in the corresponding training. Additionally, since the data is not involved in the Training-Test part, the BDT for data is obtained as the average of the five BDT scores. This choice allows to reduce the statistical fluctuations related to the differences of the five training sets. For the $\ell \tau_{\text {had }}$ channel, the data was split into $80 \%$ for training, $10 \%$ for validation and $10 \%$ for testing and a 10-fold was used so that all the available statistics were used in every step.

Correlations between the input variables of the BDT discriminant have been carefully checked, highly correlated variables have been removed and the remaining ones are ranked according to their discrimination power $[125,126]$. The list of variables is then optimized, removing the lowest-ranked variables with marginal contribution to the sensitivity. The ranking of the input variables for $\ell \tau_{\text {had }}$ channels is shown in Table 8.4. The final list of variables is presented in Table 8.5 for each channel and category. 


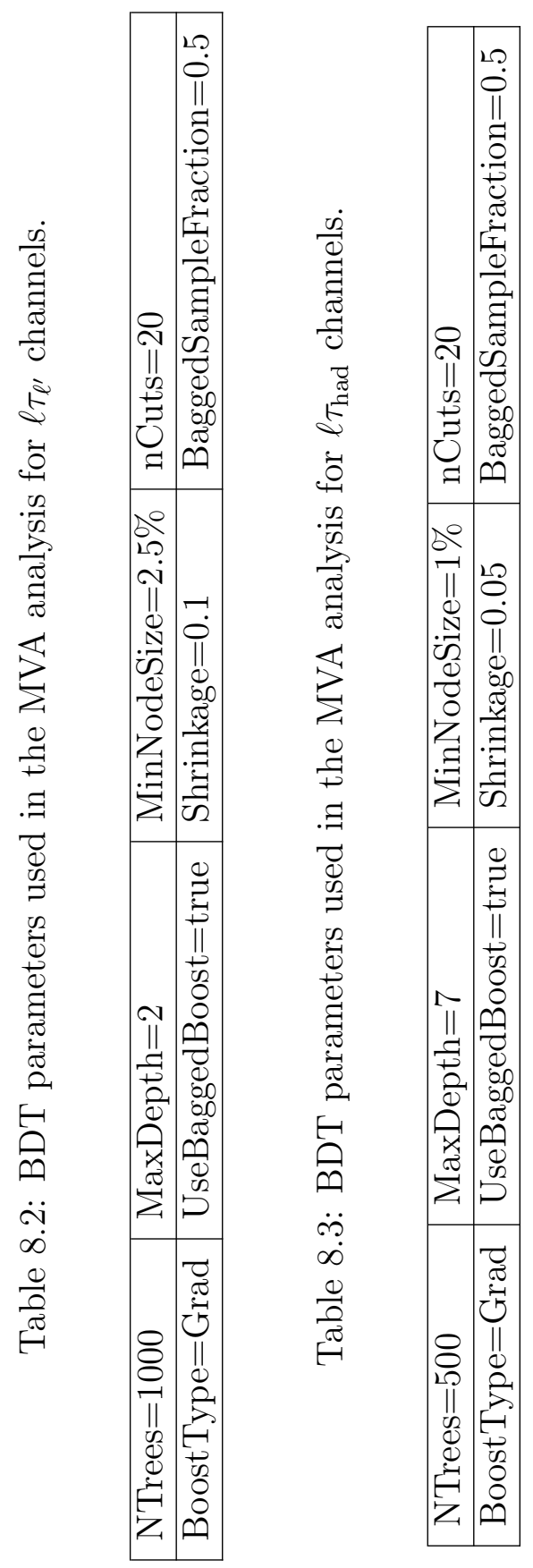


Table 8.4: MVA input variables importance $\ell \tau_{\text {had }}$ channels.

\begin{tabular}{|c|c|c|c|c|}
\hline & \multicolumn{2}{|c|}{$e \tau_{\text {had }}$} & \multicolumn{2}{|c|}{$\mu \tau_{\text {had }}$} \\
\hline & Variable & Importance & Variable & Importance \\
\hline 1 & $m_{\text {coll }}(\ell, \tau)$ & 0.602 & $m_{\text {coll }}(\ell, \tau)$ & 0.595 \\
2 & $m_{\mathrm{T}}\left(\ell, E_{\mathrm{T}}^{\text {miss }}\right)$ & 0.097 & $m_{\mathrm{T}}\left(\ell, E_{\mathrm{T}}^{\text {miss }}\right)$ & 0.098 \\
3 & $m_{\mathrm{T}}\left(\tau, E_{\mathrm{T}}^{\text {miss }}\right)$ & 0.097 & $\Delta \phi\left(\ell, E_{\mathrm{T}}^{\text {miss }}\right)$ & 0.098 \\
4 & $\Delta \phi\left(\ell, E_{\mathrm{T}}^{\text {miss }}\right)$ & 0.084 & $E_{\mathrm{T}}^{\text {miss }}$ & 0.093 \\
5 & $E_{\mathrm{T}}^{\text {miss }}$ & 0.076 & $m_{\mathrm{T}}\left(\tau, E_{\mathrm{T}}^{\text {miss }}\right)$ & 0.084 \\
6 & $m_{j j}$ & 0.070 & $p_{\mathrm{T}}(\ell)$ & 0.080 \\
7 & $p_{\mathrm{T}}(\ell)$ & 0.069 & $\Delta R(\ell, \tau)$ & 0.057 \\
8 & $\Delta \eta_{j j}$ & 0.060 & $\Delta \eta_{j j}$ & 0.057 \\
9 & $\Delta R(\ell, \tau)$ & 0.058 & $m_{j j}$ & 0.055 \\
10 & $\Delta \phi\left(\tau, E_{\mathrm{T}}^{\text {miss }}\right)$ & 0.051 & $\Sigma \cos \Delta \phi\left(\ell, E_{\mathrm{T}}^{\text {miss }}\right)$ & 0.038 \\
11 & $\Sigma \cos \Delta \phi\left(\ell, E_{\mathrm{T}}^{\text {miss }}\right)$ & 0.031 & $\Delta \phi\left(\tau, E_{\mathrm{T}}^{\text {miss }}\right)$ & 0.037 \\
12 & $p_{\mathrm{T}}(\tau)$ & 0.019 & $p_{\mathrm{T}}(\tau)$ & 0.019 \\
\hline
\end{tabular}


Table 8.5: BDT input variables used in the analysis. For each channel and category, used input variables are marked with HR (indicating the five variables with the highest rank) or a bullet. Analogous variables between the two channels are listed on the same line.

\begin{tabular}{|c|c|c|c|c|c|}
\hline \multicolumn{3}{|c|}{$\ell \tau_{\ell^{\prime}}$} & \multicolumn{3}{|c|}{$\ell \tau_{\text {had }}$} \\
\hline Variable & $\mathrm{VBF}$ & non-VBF & Variable & VBF & non-VBF \\
\hline $\begin{array}{c}m_{\mathrm{MMC}} \\
p_{\mathrm{T}}^{\ell_{1}} \\
p_{\mathrm{T}}^{\ell_{2}} \\
\Delta R\left(\ell_{1}, \ell_{2}\right) \\
m_{\mathrm{T}}\left(\ell_{1}, E_{\mathrm{T}}^{\mathrm{miss}}\right) \\
m_{\mathrm{T}}\left(\ell_{2}, E_{\mathrm{T}}^{\mathrm{miss}}\right) \\
\Delta \phi\left(\ell_{1}, E_{\mathrm{T}}^{\mathrm{miss}}\right) \\
\Delta \phi\left(\ell_{2}, E_{\mathrm{T}}^{\mathrm{miss}}\right) \\
m_{j j} \\
\Delta \eta\left(\mathrm{j}_{1}, \mathrm{j}_{2}\right) \\
p_{\mathrm{T}}^{\tau} / p_{\mathrm{T}}^{\ell_{1}}\end{array}$ & $\begin{array}{c}\mathrm{HR} \\
\bullet \\
\mathrm{HR} \\
\mathrm{HR} \\
\bullet \\
\mathrm{HR} \\
\bullet \\
\bullet \\
\mathrm{HR}\end{array}$ & $\begin{array}{c}\mathrm{HR} \\
\bullet \\
\mathrm{HR} \\
\bullet \\
\mathrm{HR} \\
\bullet \\
\bullet \\
\mathrm{HR}\end{array}$ & $\begin{array}{c}m_{\text {coll }} \\
p_{\mathrm{T}}^{\ell} \\
p_{\mathrm{T}}^{\tau_{\text {had-vis }}} \\
\Delta R\left(\ell, \tau_{\text {had-vis }}\right) \\
m_{\mathrm{T}}\left(\ell, E_{\mathrm{T}}^{\text {miss }}\right) \\
m_{\mathrm{T}}\left(\tau_{\text {had-vis }}, E_{\mathrm{T}}^{\text {miss }}\right) \\
\Delta \phi\left(\ell, E_{\mathrm{T}}^{\text {miss }}\right) \\
\Delta \phi\left(\tau_{\text {had-vis }}, E_{\mathrm{T}}^{\text {miss }}\right) \\
m_{j j} \\
\Delta \eta\left(\dot{\mathrm{j}}_{1}, \mathrm{j}_{2}\right) \\
\sum \cos \Delta \phi\left(i, E_{\mathrm{T}}^{\mathrm{miss}}\right) \\
i=\ell, \tau_{\text {had-vis }} \\
E_{\mathrm{T}}^{\text {miss }} \\
m_{\text {vis }} \\
\Delta \eta\left(\ell, \tau_{\text {had-vis }}\right) \\
\eta^{\ell} \\
\eta^{\tau_{\text {had-vis }}} \\
\phi^{\ell} \\
\phi^{\tau_{\text {had-vis }}} \\
\phi\left(E_{\mathrm{T}}^{\text {miss }}\right)\end{array}$ & $\begin{array}{c}\mathrm{HR} \\
\bullet \\
\bullet \\
\bullet \\
\mathrm{HR} \\
\mathrm{HR} \\
\mathrm{HR} \\
\bullet \\
\bullet \\
\bullet \\
\bullet \\
\mathrm{HR}\end{array}$ & $\begin{array}{c}\bullet \\
\bullet \\
\mathrm{HR} \\
\bullet \\
\bullet \\
\bullet \\
\bullet \\
\bullet \\
\bullet\end{array}$ \\
\hline
\end{tabular}




\subsection{Event Selection and Categorization}

Selected events in the analysis are triggered with single-electron or single-muon triggers. The $p_{\mathrm{T}}$ thresholds depend on the isolation requirement and data-taking period $[127,128]$. The lowest trigger thresholds correspond to $25-27 \mathrm{GeV}$ for electrons and $21-27 \mathrm{GeV}$ for muons.

Events selected in the $\ell \tau_{\ell^{\prime}}$ channel contain exactly one electron and one muon of opposite charge (OS). Similarly in the $\ell \tau_{\text {had }}$ channel, a lepton and a $\tau_{\text {had-vis }}$ of opposite charge are required, and events with more than one lepton are rejected.

The selection criteria are summarized in Table 8.6 for the analysis categories as well as the control regions (CRs), which are described in Section 8.5.

In the $\ell \tau_{\ell^{\prime}}$ channel, $\ell_{1}$ and $\ell_{2}$ denote the leading and subleading lepton in $p_{\mathrm{T}}$, respectively. Events where the leading lepton is an electron (muon) are used in the search for $H \rightarrow e \tau_{\mu}\left(H \rightarrow \mu \tau_{e}\right)$. A requirement on the dilepton invariant mass, equal to the invariant mass of the lepton and the visible $\tau$-decay products, $m_{\mathrm{vis}}$, reduces backgrounds with top quarks, and the criterion applied to the track-to-cluster $p_{\mathrm{T}}$ ratio of the electron reduces the $Z \rightarrow \mu \mu$ background where a muon deposits a large amount of energy in the electromagnetic calorimeter and is misidentified as an electron in the $\mu \tau_{e}$ channel. The contribution from the $H \rightarrow \tau \tau$ decay is reduced by the asymmetric $p_{\mathrm{T}}$ selection of the two leptons.

In the $\ell \tau_{\text {had }}$ channel, the criterion based on the azimuthal separations of lepton$E_{\mathrm{T}}^{\mathrm{miss}}$ and $\tau_{\text {had-vis }}-E_{\mathrm{T}}^{\mathrm{miss}}, \sum_{i=\ell, \tau_{\text {had-vis }}} \cos \Delta \phi\left(i, E_{\mathrm{T}}^{\mathrm{miss}}\right)$, reduces the $W+$ jets background whereas the requirement on $\left|\Delta \eta\left(\ell, \tau_{\text {had-vis }}\right)\right|$ reduces backgrounds with misidentified $\tau_{\text {had-vis }}$ candidates.

For both channels of each search, a $b$-veto requirement reduces the single-topquark and $t \bar{t}$ backgrounds. Events are further categorized into VBF (with a focus on the VBF production of the Higgs boson) and non-VBF categories. The VBF 
selection is based on the kinematics of the two jets with the highest $p_{\mathrm{T}}$, where $\mathrm{j}_{1}$ and $\mathrm{j}_{2}$ denote the leading and subleading jet in $p_{\mathrm{T}}$, respectively. The variables $m_{j j}$ and $\Delta \eta\left(\mathrm{j}_{1}, \mathrm{j}_{2}\right)$ stand for the invariant mass and $\eta$ separation of these two jets. The non-VBF category contains events failing the VBF selection.

In the dilepton channel, additional selection criteria are applied to further reject background events in this category. These criteria are also listed in Table 8.6, where $m_{\mathrm{T}}$ stands for the transverse mass ${ }^{3}$ of the two objects listed in parentheses, and $p_{\mathrm{T}}^{\tau}$ represents the magnitude of the vector sum of $p_{\mathrm{T}}^{\ell_{2}}$ and $E_{\mathrm{T}}^{\mathrm{miss}}$. The requirement on $p_{\mathrm{T}}^{\tau} / p_{\mathrm{T}}^{\ell_{1}}$ reduces the background arising from jets misidentified as leptons.

The VBF and non-VBF categories in each of the $\ell \tau_{\ell^{\prime}}$ and $\ell \tau_{\text {had }}$ channels give rise to four signal regions in each search.

\footnotetext{
${ }^{3}$ The transverse mass of two objects is defined as $m_{\mathrm{T}}=\sqrt{2 p_{\mathrm{T} 1} p_{\mathrm{T} 2}(1-\cos \Delta \phi)}$, where $p_{\mathrm{T} i}$ are the individual transverse momenta and $\Delta \phi$ is the angle between the two objects in the azimuthal plane.
} 


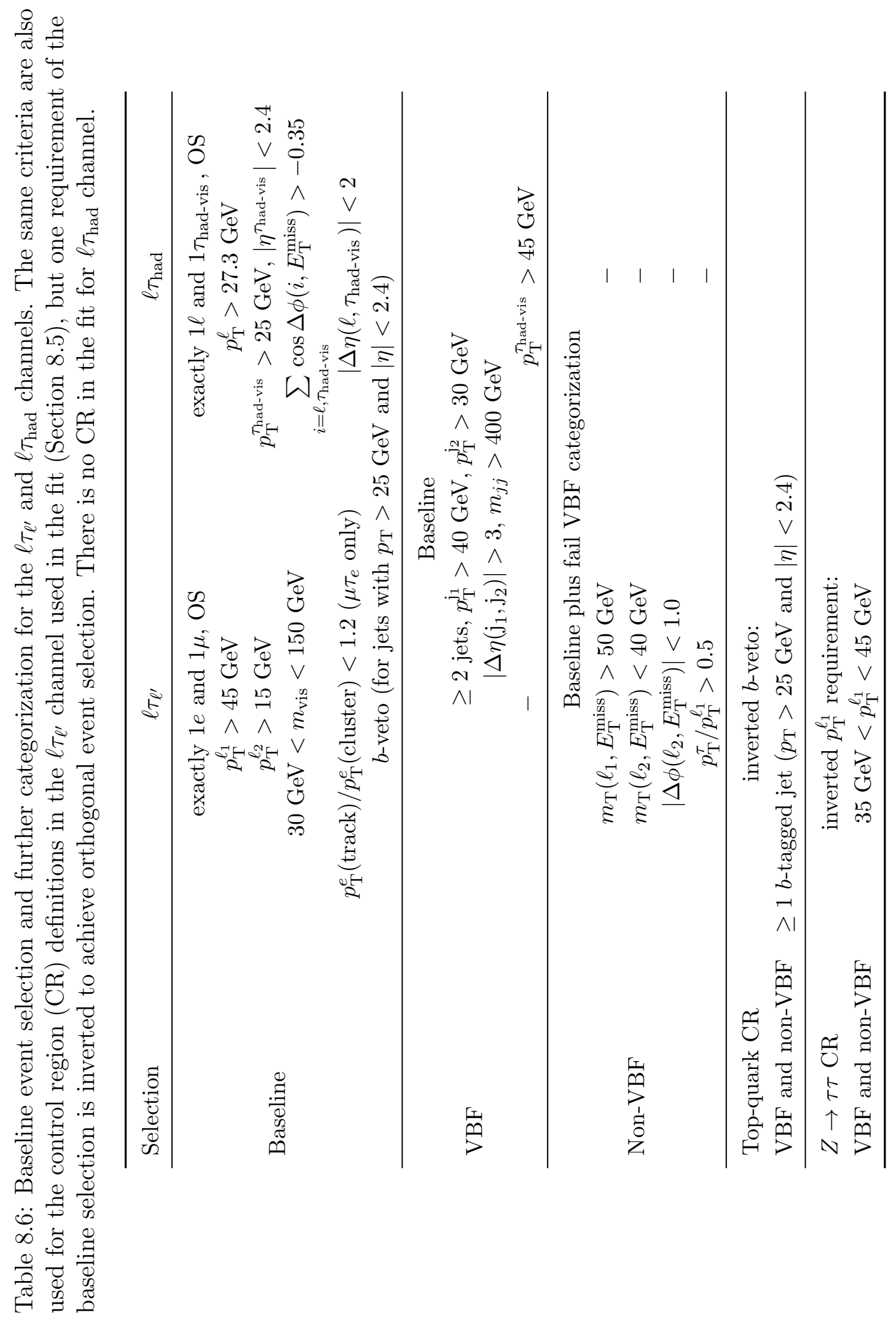


The cut-based analysis employs an estimator of the Higgs boson mass for the signal extraction. The MMC is used in $\ell \tau_{\ell^{\prime}}$ channel, while the collinear mass approximation is exploited in the $\ell \tau_{\text {had }}$ final state.

The categorization of the cut-based analysis in the $\ell \tau_{\ell^{\prime}}$ channel is the same as for the MVA analysis. In the $\ell \tau_{\text {had }}$ channel, the VBF definition is also the same, while the non-VBF category is further split into three orthogonal signal regions (SR1-SR3). The orthogonal signal regions have been defined to select different signal topologies as shown in Figure 8.3. Definitions of these signal regions as well as the control regions used for background modelling as discussed in Section 8.5 are presented in Tables 8.7, 8.8 and 8.9. The cut-based approach gives results consistent with no LFV signal, although not as sensitive as the MVA analysis.

Table 8.7: Definition of the categories of the $\ell \tau_{\text {had }}$ channel for the cut-based analysis.

\begin{tabular}{|c|c|}
\hline Region & Requirements \\
\hline VBF & $\begin{array}{c}p_{\mathrm{T}}^{\tau_{\text {had-vis }}}>45 \mathrm{GeV}, N_{\text {jets }}\left(p_{\mathrm{T}}>30 \mathrm{GeV}\right) \geq 2, p_{\mathrm{T}}^{\text {leading jet }}>40 \mathrm{GeV} \\
m_{j j}>400 \mathrm{GeV},\left|\Delta \eta\left(\mathrm{j}_{1}, \mathrm{j}_{2}\right)\right|>3\end{array}$ \\
\hline SR1 & $p_{\mathrm{T}}^{\tau_{\text {had-vis }}}>45 \mathrm{GeV}, m_{\mathrm{T}}\left(\ell, E_{\mathrm{T}}^{\text {miss }}\right)>40 \mathrm{GeV}, m_{\mathrm{T}}\left(\tau_{\text {had-vis }}, E_{\mathrm{T}}^{\text {miss }}\right)<30 \mathrm{GeV}$, fail VBF \\
\hline SR2 & $p_{\mathrm{T}}^{\tau_{\text {had-vis }}}>45 \mathrm{GeV}, m_{\mathrm{T}}\left(\ell, E_{\mathrm{T}}^{\text {miss }}\right)<40 \mathrm{GeV}, m_{\mathrm{T}}\left(\tau_{\text {had-vis }}, E_{\mathrm{T}}^{\text {miss }}\right)<60 \mathrm{GeV}$, fail VBF \\
\hline SR3 & $\begin{array}{c}25 \mathrm{GeV}<p_{\mathrm{T}}^{\tau_{\mathrm{T} a d-v i s}}<45 \mathrm{GeV}, \\
m_{\mathrm{T}}\left(\ell, E_{\mathrm{T}}^{\mathrm{miss}}\right)>40 \mathrm{GeV}, m_{\mathrm{T}}\left(\tau_{\text {had-vis }}, E_{\mathrm{T}}^{\mathrm{miss}}\right)<30 \mathrm{GeV} \\
p_{\mathrm{T}}^{\ell}>45 \mathrm{GeV}, \text { fail VBF }\end{array}$ \\
\hline
\end{tabular}
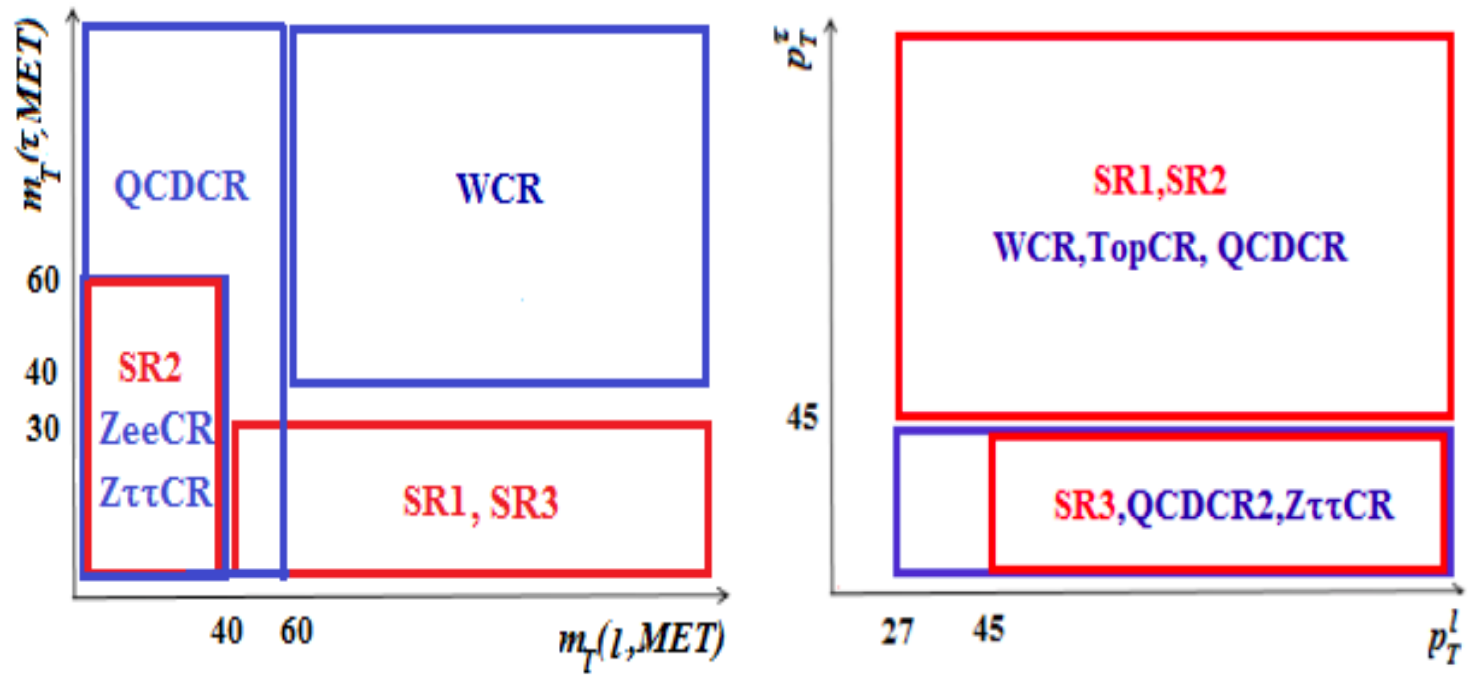

Figure 8.3: Sketches for different $\mathrm{SR}$ and $\mathrm{CR}$ regions in $\mathrm{CBA}$ for $\ell \tau_{\text {had }}$. 
Table 8.8: Summary of the event selection for the $\ell \tau_{\text {had }}$ QCD control regions for the fake factor method.

\begin{tabular}{|c|c|}
\hline & $\ell \tau_{\text {had }}$ \\
\hline & $e \tau_{\text {had }}$ \\
\hline $\begin{array}{l}\text { QCD CR } \\
\text { baseline }\end{array}$ & $\begin{array}{c}\text { Baseline selection } \\
\text { except }\left|\Delta \eta\left(\ell, \tau_{\text {had-vis }}\right)\right| \\
\left|\Delta \eta\left(\ell, \tau_{\text {had-vis }}\right)\right|>2 \\
m_{\mathrm{T}}\left(\ell, E_{\mathrm{T}}^{\text {miss }}\right)<60 \mathrm{GeV}\end{array}$ \\
\hline QCD CR1 & $p_{\mathrm{T}}\left(\tau_{\text {had-vis }}\right)>45 \mathrm{GeV}$ \\
\hline$\overline{\mathrm{Q}} \overline{\mathrm{CD}} \overline{\mathrm{CR}} 2$ & $\begin{array}{c}p_{\mathrm{T}}\left(\tau_{\text {had-vis }}\right)>45 \mathrm{GeV} \\
m_{\mathrm{T}}\left(\ell, E_{\mathrm{T}}^{\text {miss }}\right)<40 \mathrm{GeV} \\
m_{\mathrm{T}}\left(\tau_{\text {had-vis }}, E_{\mathrm{T}}^{\text {miss }}\right)<60 \mathrm{GeV}\end{array}$ \\
\hline$\overline{\mathrm{Q}} \overline{\mathrm{C}} \overline{\mathrm{CR}} \overline{\mathrm{CR}}$ & $25 \mathrm{GeV}<p_{\mathrm{T}}\left(\tau_{\text {had-vis }}\right)<\overline{4} \overline{\mathrm{Ge}} \overline{\mathrm{e}}$ \\
\hline$\overline{\mathrm{QCD}} \overline{\mathrm{C}} \overline{\mathrm{R}} \overline{\mathrm{V}} \overline{\mathrm{F}}$ & VBF selection \\
\hline$\overline{\mathrm{QCD}} \overline{\mathrm{MVA}} \overline{\mathrm{M}}$ & same as QCD baseline \\
\hline
\end{tabular}

Table 8.9: Summary of the event selection for the $\ell \tau_{\text {had }} W+$ jets control regions for the fake factor method.

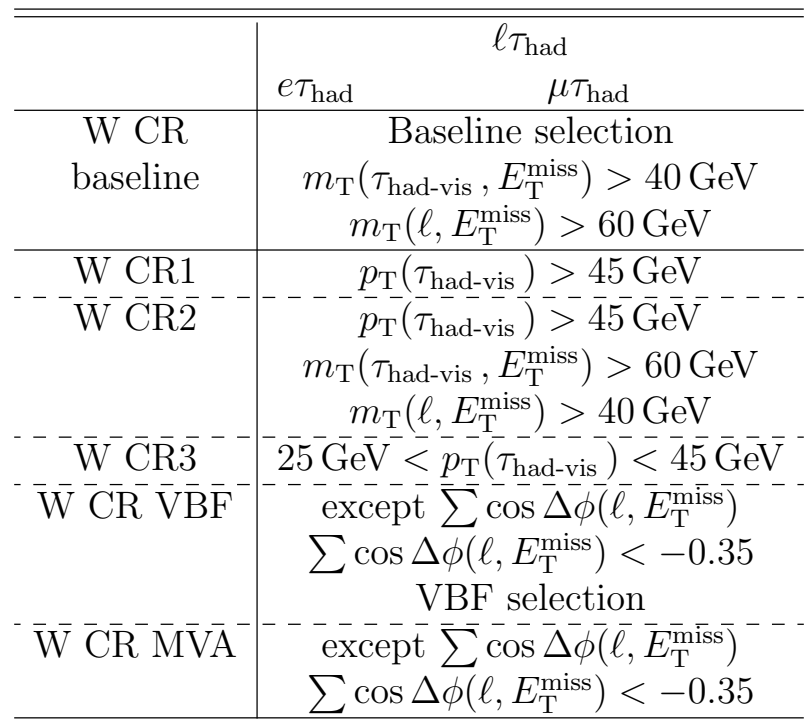




\subsection{Background modelling}

The most significant backgrounds in the search are from events with $Z \rightarrow \tau \tau$ decays or with (single or pair-produced) top quarks, especially in the $\ell \tau_{\ell^{\prime}}$ channel, as well as from events with misidentified objects, which are estimated using data-driven techniques. The relative contribution from misidentified objects to the total background yield is $5-25 \%$ in the $\ell \tau_{\ell^{\prime}}$ channel and $25-45 \%$ in the $\ell \tau_{\text {had }}$ channel, depending on the search and the analysis category. The shapes of distributions from the $Z \rightarrow \tau \tau$ and top-quark (single-top-quark and $t \bar{t}$ ) processes are modelled by simulation in both the $\ell \tau_{\ell^{\prime}}$ and $\ell \tau_{\text {had }}$ decay channels.

In the $\ell \tau_{\ell^{\prime}}$ channel, the relative contributions of $Z \rightarrow \tau \tau$ and top-quark production processes are $20-35 \%$ and $20-55 \%$, respectively; the top-quark background dominates in the VBF category. In the $\ell \tau_{\text {had }}$ channel, the top-quark background fraction is $1-$ $10 \%$, while the $Z \rightarrow \tau \tau$ process contributes to $45-55 \%$ of the total background. Smaller background components are also modelled by simulation and are grouped together: $Z \rightarrow \mu \mu$, diboson production, $H \rightarrow \tau \tau$ and $H \rightarrow W W$.

Details of the background estimation techniques are given below.

\subsection{1 $\ell \tau_{\ell^{\prime}}$ channel}

Two sets of CRs, as defined in Table 8.6, are used to constrain the normalization of $Z \rightarrow \tau \tau$ and top-quark background components. These CRs inherit their definitions from the corresponding analysis category but invert one requirement to ensure orthogonality with the nominal selection. The normalization factors are determined during the statistical analysis by fitting the event yields in all signal and control regions simultaneously. For each search, separate $Z \rightarrow \tau \tau$ normalization factors are used for the VBF and non-VBF categories. In the case of the top-quark background, in which leading jets are produced at a lower order of the perturbative expansion of 
the scattering process, a combined normalization factor across the two categories is used in the $\ell \tau_{\ell^{\prime}}$ channel.

Top-quark CRs are almost exclusively composed of top-quark backgrounds: the purity is $95 \%$ across both searches and categories, with $t \bar{t}$ process accounting for more than $90 \%$ of the top-quark backgrounds. The $Z \rightarrow \tau \tau$ CRs achieved a purity of $\sim 80 \%$ in the non-VBF categories, while a lower purity of $\sim 60 \%$ is observed in the VBF categories. The contributions of all other background components are normalized to their SM predictions when the likelihood fit (Section 8.7) is applied.

The shape and normalization of diboson and $Z \rightarrow \mu \mu$ background distributions are validated with data in dedicated regions where their contributions are enhanced. The latter process only contributes sizeably in the $\mu \tau_{e}$ channel, where it represents up to $10 \%$ of the total background. Another source of background comes from $W+$ jets, topquark and multi-jet events, where jets are misidentified as leptons. This background is estimated directly from OS data events where an inverted isolation requirement is imposed on the subleading lepton [83]. Normalization factors are applied to correct for the inverted isolation requirement. The normalization factors are derived in a dedicated region where the leptons are required to have same-sign (SS) charges. Additional corrections are made by reweighting the $\mathrm{MC}$ distributions of $\Delta \phi\left(\ell_{1}, E_{\mathrm{T}}^{\mathrm{miss}}\right)$ and $\Delta \phi\left(\ell_{2}, E_{\mathrm{T}}^{\mathrm{miss}}\right)$ to data in the SS region, which improves the modelling of azimuthal angles between leptons and the $E_{\mathrm{T}}^{\text {miss }}$ direction as well as the modelling of $m_{\mathrm{T}}\left(\ell_{2}, E_{\mathrm{T}}^{\mathrm{miss}}\right)$. A similar improvement is observed in the nominal OS region. In most of the cases, the misidentified jet mimics the lepton of lower $p_{\mathrm{T}}, \ell_{2}$, while the fraction of events where both leptons are misidentified varies between $2 \%$ to $8 \%$ across categories. The systematic uncertainties of the estimation of the misidentified lepton background include contributions from closure tests in SS and OS regions enriched with misidentified leptons, from the corrections made to the $\Delta \phi$ distributions, and from the composition of the misidentified lepton background. 


\subsection{2 $\ell \tau_{\text {had }}$ channel}

The main background contributions come from the $Z \rightarrow \tau \tau$ process and events where either a jet or an electron is misidentified as $\tau_{\text {had-vis }}$. The shape of the $Z \rightarrow \tau \tau$ background distribution is modelled by simulation, and the corresponding normalization factors are determined from the simultaneous fit (Section 8.7) of the event yields in all signal and control regions. The $Z \rightarrow \tau \tau$ normalization factors are fully correlated with those of the $\ell \tau_{\ell^{\prime}}$ channel, in each VBF and non-VBF category. Top-quark production represents less than $1 \%$ of the total background in the $\ell \tau_{\text {had }}$ channel and is determined by simulation, including its normalization, which is kept fixed in the fit.

The main contributions to jets misidentified as $\tau_{\text {had-vis }}$ come from multi-jet events and $W$-boson production in association with jets, and a fake-factor method is used to estimate the contribution of each component separately, as described below in Sections 8.5.2.1 through 8.5.2.3. A fake factor is defined as the ratio of the number of events where the highest- $p_{\mathrm{T}}$ jet is identified as a tight $\tau_{\text {had-vis }}$ candidate to the number of events with a highest- $p_{\mathrm{T}}$ jet failing this tau-ID criterion but satisfying a looser one.

The fake-factor method is also adopted to estimate the electron-faking $\tau_{\text {had-vis }}$ backgrounds, as described in Sections 8.5.2.4 through 8.5.2.6. Finally validation of backgrounds with muons are presented in Section 8.5.2.7.

\subsubsection{Jet-fake background estimation}

The background which has jets faking $\tau_{\text {had-vis }}$ leptons is a dominant background in the LFV $H \rightarrow \ell \tau_{\text {had }}$ channel. The $\tau_{\text {had-vis }}$-fake background is estimated using a control region (called "anti-identified" region) in which the lepton satisfies the same identification and isolation criteria as signal region (called "identified" region), while

the $\tau_{\text {had-vis }}$ fails the "tight" requirement but satisfies a looser criteria. The dominant contribution to this background comes from $\mathrm{W}+$ jets and multi-jet events in which a 
jet produces an object which is reconstructed as a $\tau_{\text {had-vis }}$. Other smaller contributions come from top-quark production (single top and $t \bar{t}$ ) and $\mathrm{Z}+$ jets events.

The $\tau_{\text {had-vis }}$-fake background in the anti-ID region is extrapolated to the signal region by a "fake factor" $F F$.

$$
N_{j \rightarrow \tau}^{\mathrm{ID}}=\left(N_{\mathrm{data}}^{\mathrm{anti}-\mathrm{ID}}-N_{\mathrm{MC}, \text { not } \rightarrow \tau}^{\mathrm{anti}-\mathrm{ID}}\right) \times F F
$$

Since we have more than one source of $\tau_{\text {had-vis }}$-fake background, the fake factor is therefore factorized into individual components $\left(F F_{i}\right)$ for each relevant process $i$. The combined fake factor is then constructed as the sum of components weighted by the fractional contributions from the relevant process in the anti-ID region $\left(R_{i}\right)$

$$
F F=\sum_{i} R_{i} \times F F_{i}
$$

In practice, the contributions from top-quark production and $\mathrm{Z}+$ jets are rather small and do not play important roles and can be considered to be similar to the fake factor of the $\mathrm{W}+$ jets background. Therefore, we can simplify the above equation to

$$
F F=R_{\mathrm{W}} \times F F_{\mathrm{W}}+R_{\mathrm{QCD}} \times F F_{\mathrm{QCD}}
$$

The relative contribution $R_{\mathrm{QCD}}$ of multi-jet events is obtained by a data-driven method, which will be described in the subsequent section, and the corresponding $R_{\mathrm{W}}$ is obtained as $R_{\mathrm{W}}=1-R_{\mathrm{QCD}}$.

The individual fake factors are determined in dedicated W and QCD control regions, respectively. These control regions are all defined to be the same as the $\mathrm{W}$ and QCD control regions (WCR and QCDCR1) in Section 8.4. The individual FF 
is defined as the ratio of identified to anti-identified numbers of events

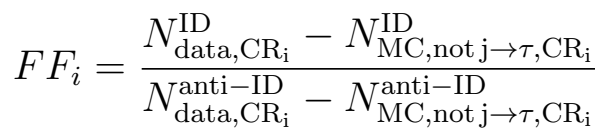

$F F_{i}$ is estimated in each signal region as a function of the $\tau_{\text {had-vis }} p_{\mathrm{T}}$ and the number of prongs of the $\tau_{\text {had-vis }}$ decay, as shown in Figure 8.4 , because, among the $\tau_{\text {had-vis }}$ kinematic variables, the fake factors show the greatest dependence versus $\tau_{\text {had-vis }} p_{\mathrm{T}}$.

The same procedure is also applied in order to obtain the fake background in the validation regions used in this analysis using Top VR and $\mathrm{Z} \tau \tau \mathrm{VR}$ as described in Section 8.4.

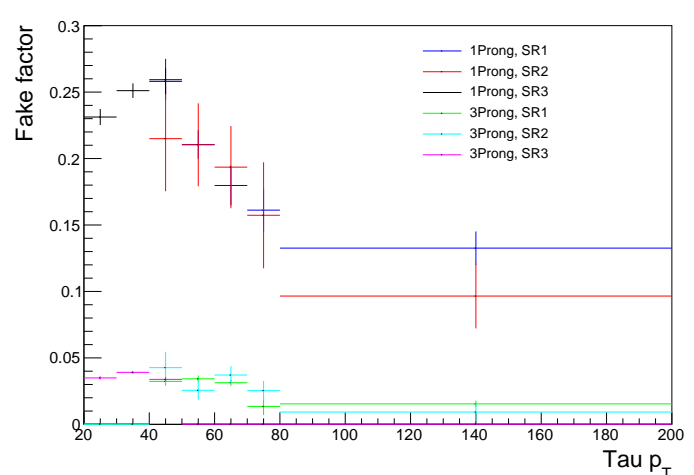

(a)

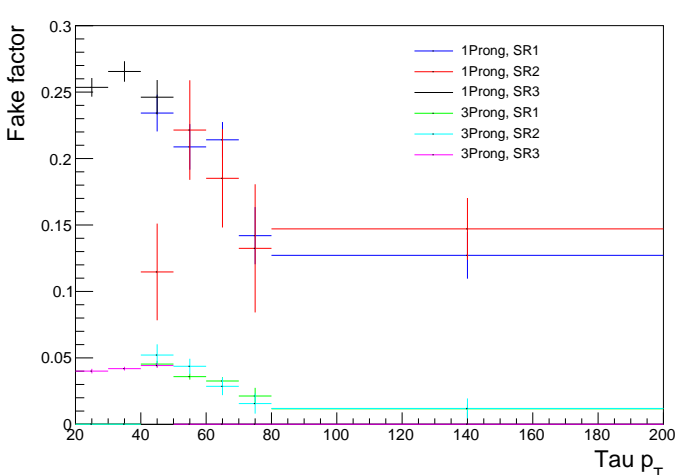

(b)

Figure 8.4: Distributions of fake factors in each signal region (SR1, SR2 and SR3) as a function of the $\tau_{\text {had-vis }} p_{T}$ and the number of prongs of the $\tau_{\text {had-vis }}$ decay for (a) $H \rightarrow \mu \tau_{\text {had }}$ and (b) $H \rightarrow e \tau_{\text {had }}$ channels.

\subsubsection{Determination of $R_{\mathrm{QCD}}$}

The relative contribution $R_{\mathrm{QCD}}$ of multi-jet events is given by

$$
R_{\mathrm{QCD}}=\frac{N_{\mathrm{QCD}, \text { data }}^{\mathrm{anti}-\mathrm{ID}}}{N_{\text {data }}^{\text {anti-ID }}-N_{\mathrm{MC}, \text { not } \mathrm{j} \rightarrow \tau}^{\text {anti-ID }}}
$$

The $N_{\mathrm{QCD} \text {,data }}^{\text {anti-ID }}$ is estimated from data by multiplying the events in the "anti-Iso" CR (by inverting the isolation requirement on the lepton) with a transfer factor, called 
"isolation factors" $(I F)$ accounting for the difference between failing and passing the lepton isolation.

$$
N_{\mathrm{QCD}, \text { data }}^{\mathrm{anti}-\mathrm{ID}}=\left(N_{\mathrm{data}}^{\text {anti } \mathrm{so}, \text { anti-ID }}-N_{\mathrm{MC}, \text { true lepton }}^{\text {anti } \mathrm{I}_{\mathrm{S}}, \text { anti-ID }}\right) \times I F
$$

The isolation factor is calculated as the ratio of the events passing and failing the lepton isolation requirement in a dedicated control region. This control region has the same cuts as the preselection but requires the signs of the $\tau_{\text {had-vis }}$ candidate and the lepton to be the same, rather than opposite (denoted as "SSP" region). The isolation factors are calculated separately for electrons and muons and binned in $p_{\mathrm{T}}$ and $|\eta|$ of the lepton, as shown in Figure 8.5.

$$
I F=\frac{N_{\text {data,SSP }}^{\text {Iso,anti-ID }}-N_{\mathrm{MC}, \text { true lepton,SSP }}^{\text {Iso,anti-ID }}}{N_{\text {data,SSP }}^{\text {anti-Iso,anti-ID }}-N_{\text {MC,true lepton,SSP }}^{\text {anti-Iso,antid }}}
$$

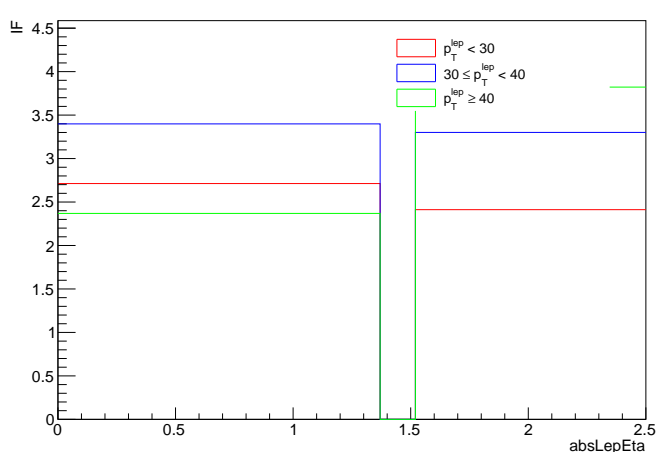

(a)

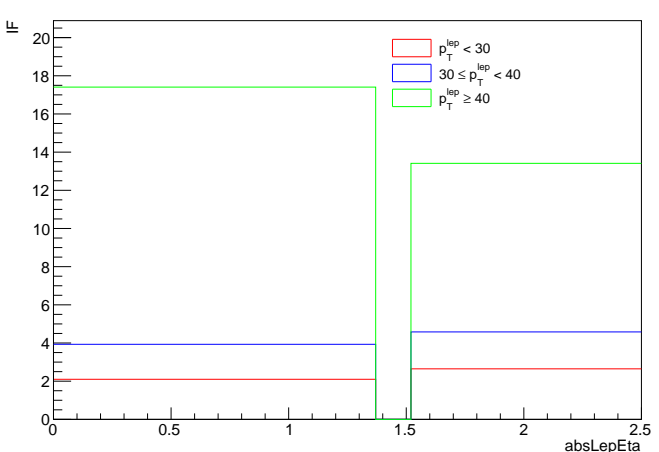

(b)

Figure 8.5: Isolation factors for muons (left) and electrons (right) as a function of the lepton's $p_{\mathrm{T}}$ and $|\eta|$.

\subsubsection{Closure tests of jet fakes}

In order to show that the method performs as expected, the same procedure was repeated with the same-sign region instead of the opposite-sign one, using exactly 
the same setup and selection as for the standard analysis. The results are shown in Figures 8.6 and 8.7, respectively.

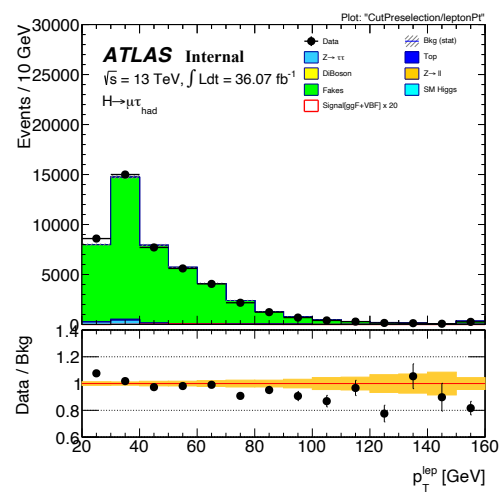

(a) $p_{\mathrm{T}}(\ell)$

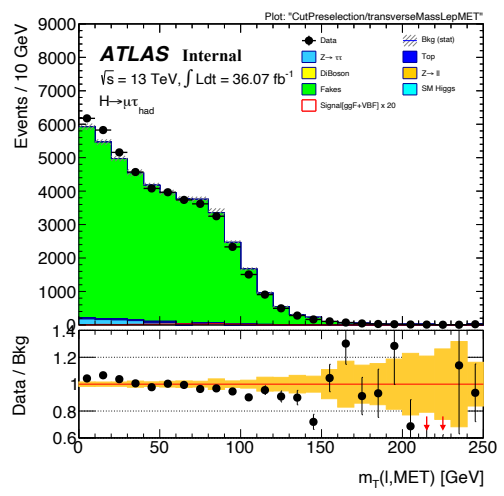

(d) $m_{\mathrm{T}}\left(\ell, E_{\mathrm{T}}^{\mathrm{miss}}\right)$

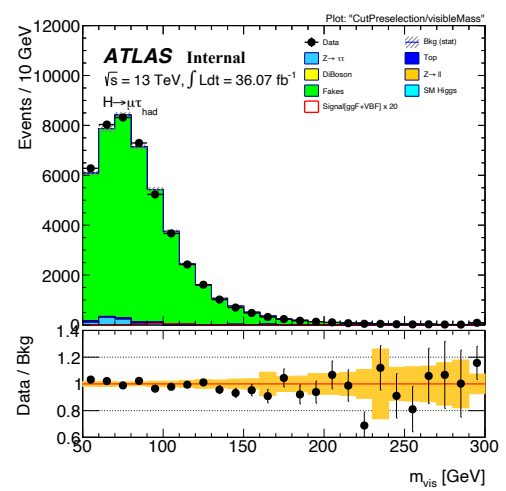

(g) $m_{\text {vis }}$

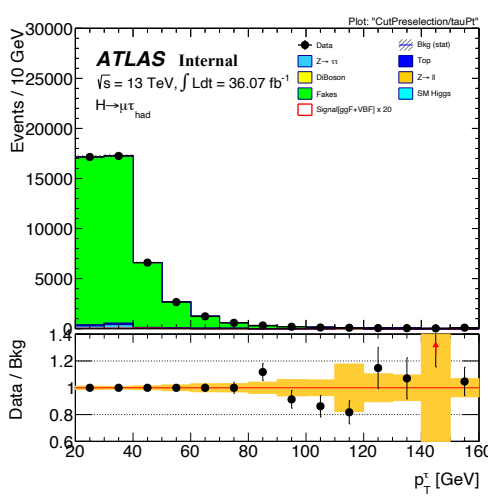

(b) $p_{\mathrm{T}}(\tau)$

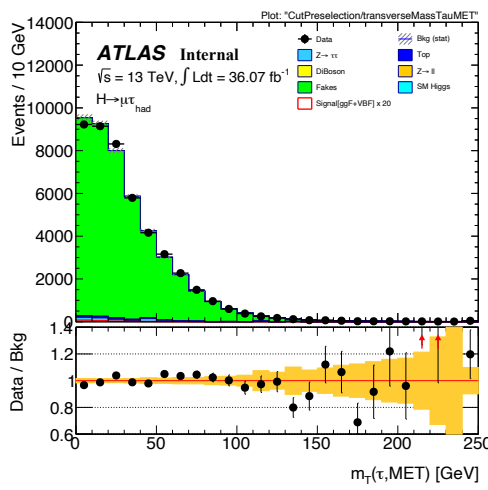

(e) $m_{\mathrm{T}}\left(\tau, E_{\mathrm{T}}^{\mathrm{miss}}\right)$

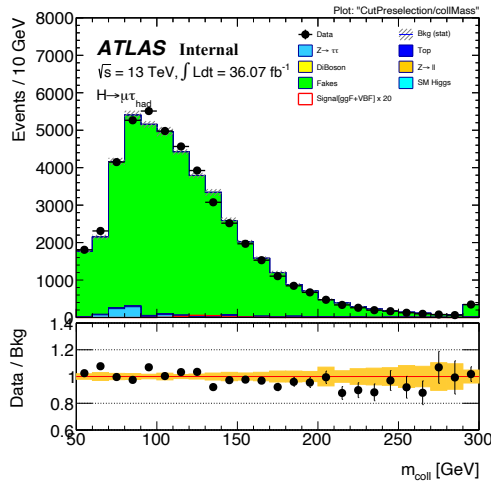

(h) $m_{\text {coll }}$

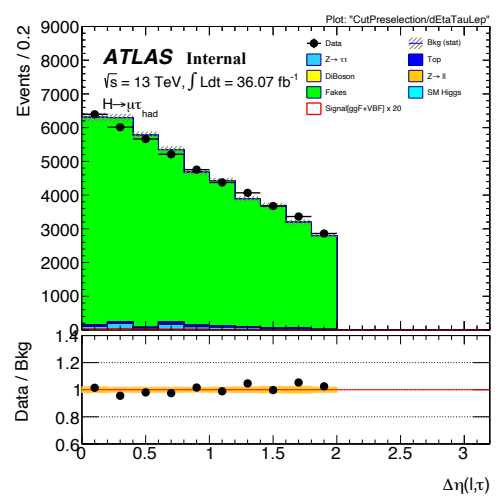

(c) $\Delta \eta(\ell, \tau)$

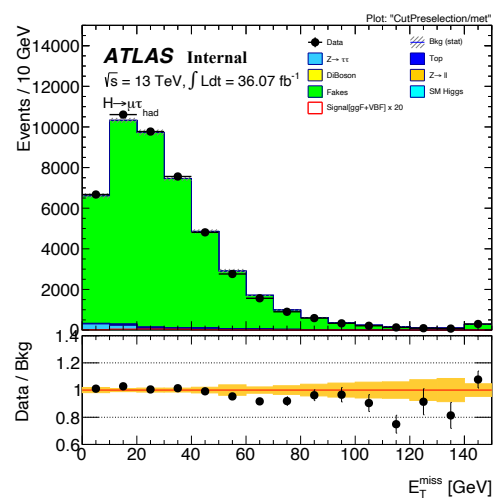

(f) $E_{\mathrm{T}}^{\text {miss }}$

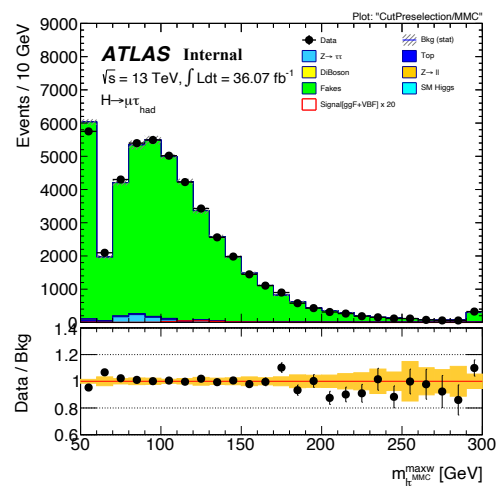

(i) $M M C$

Figure 8.6: Closure test in the preselection same sign region in $\mu \tau_{\text {had }}$ channel. The statistical uncertainties for all samples and the weight and kinematic systematics for the background are shown. 


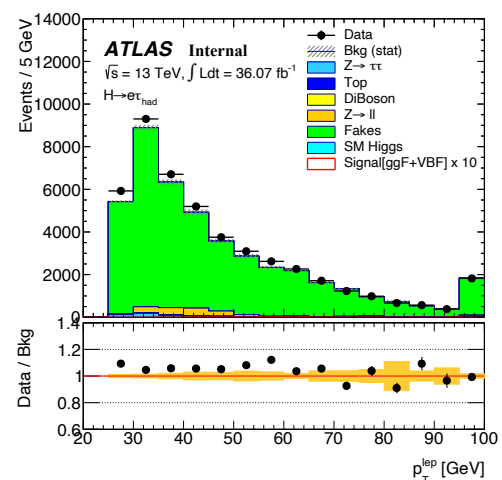

(a) $p_{\mathrm{T}}(\ell)$

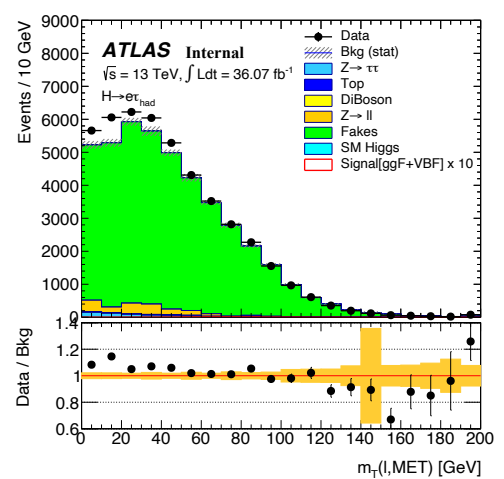

(d) $m_{\mathrm{T}}\left(\ell, E_{\mathrm{T}}^{\mathrm{miss}}\right)$

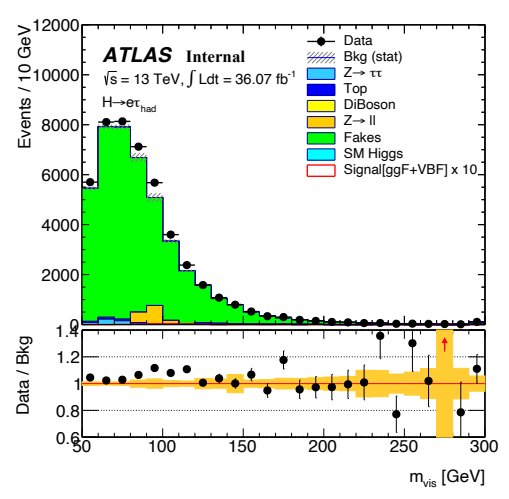

(g) $m_{\mathrm{vis}}$

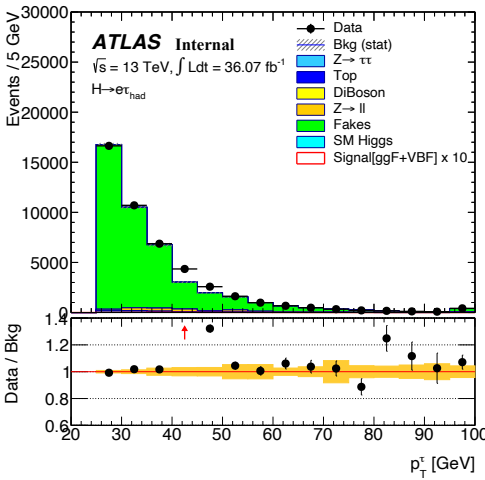

(b) $p_{\mathrm{T}}(\tau)$

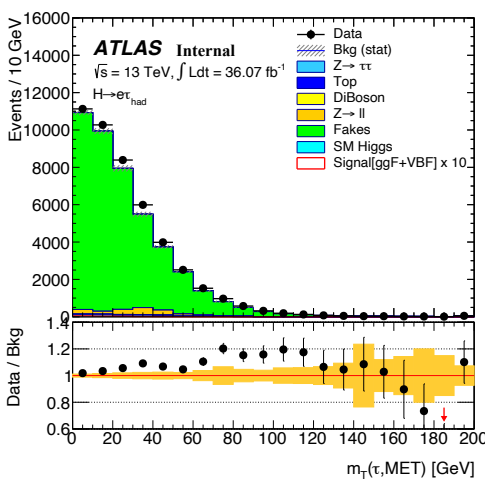

(e) $m_{\mathrm{T}}\left(\tau, E_{\mathrm{T}}^{\text {miss }}\right)$

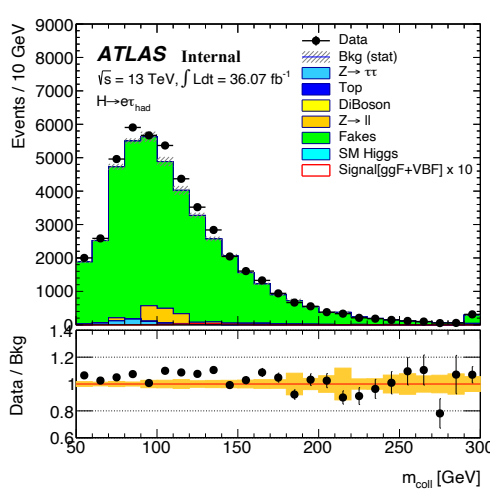

(h) $m_{\text {coll }}$

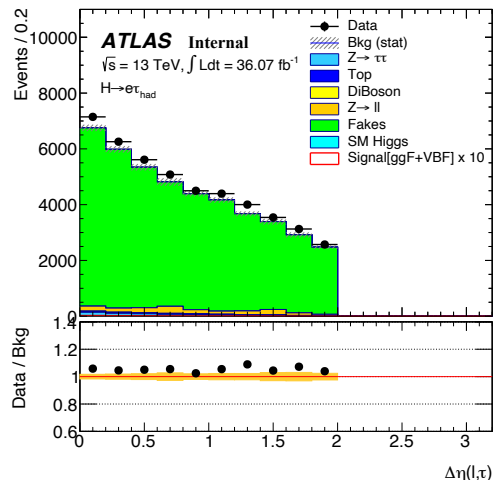

(c) $\Delta \eta(\ell, \tau)$

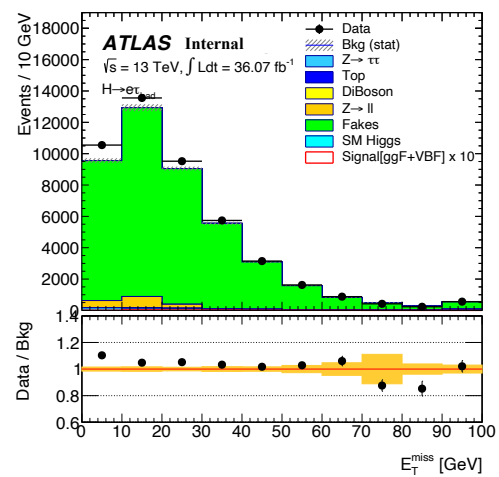

(f) $E_{\mathrm{T}}^{\mathrm{miss}}$

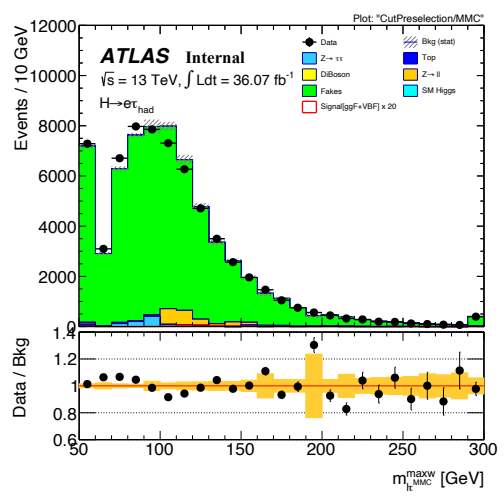

(i) $M M C$

Figure 8.7: Closure test in the preselection same sign region in $e \tau_{\text {had }}$ channel. The statistical uncertainties for all samples and the weight and kinematic systematics for the background are shown. 


\subsubsection{Electron-fake background estimation}

One of the main backgrounds in the LFV $H \rightarrow \ell \tau_{\text {had }}$ channel is $\mathrm{Z}(\rightarrow \ell \ell)+$ jet. In $\mathrm{Z}(\rightarrow \ell \ell)+$ jet, the $\tau_{\text {had-vis }}$ candidate can originate either from lepton (muon or electron) or jet. Since the jet-originated events are estimated by the data-driven method (see Section 8.5.2.1), the lepton-originated events are based on MC simulation. However, due to the lack of MC statistics, especially in the $H \rightarrow e \tau_{\text {had }}$ channel, $Z \rightarrow e e$ background is also estimated by a data-driven method. The methodology used is based on a fake factor calculation, very similar in nature to the one used for jetfaking- $\tau_{\text {had-vis }}$ background, the fake factor method. Only those $\tau_{\text {had-vis }}$ candidates with exactly one associated track are considered, as the rate of electrons faking 3track $\tau_{\text {had-vis }}$ is negligible.

Similar to the jet-faking- $\tau_{\text {had-vis }}$ background, the electron-faking- $\tau_{\text {had-vis }}$ background is estimated using an "anti-ID" region in which the $\tau_{\text {had-vis }}$ lepton satisfies all selection criteria but fails the 1 -track electron veto requirement (BDT score $>0.15$ ). Other smaller contributions in this region come from jet-faking- $\tau_{\text {had-vis }}$, top-quark production and $Z \rightarrow \tau \tau$ events.

The electron-faking- $\tau_{\text {had-vis }}$ background in the anti-ID region is extrapolated to the signal region by an "electron fake factor" (eFF)

$$
N_{e \rightarrow \tau}^{\mathrm{ID}}=\left(N_{1-\text { prong data }}^{\text {anti-ID }}-N_{1-\text { prong MC,not e } \rightarrow \tau}^{\text {anti-ID }}\right) \times e F F+N_{3-\text { prong MC,e } \rightarrow \tau}^{\mathrm{ID}}
$$

The fake factor $e F F$ is determined in a "so-called" $Z \rightarrow e e$ control region which is same as the preselection but with the tauID requirement (medium, but not tight) instead of tight, to avoid the overlap between $Z \rightarrow e e$ control region and signal regions in $\ell \tau_{\ell^{\prime}}$ analysis. In addition, some requirements are also added to enhance the contribution of $Z \rightarrow$ ee events in this region, as shown in Figure 8.3:

- $\left|m_{\mathrm{vis}}-m_{Z}\right|<5 \mathrm{GeV}$ 
- $m_{\mathrm{T}}\left(\ell, E_{\mathrm{T}}^{\mathrm{miss}}\right)<40 \mathrm{GeV}$

- $m_{\mathrm{T}}\left(\tau, E_{\mathrm{T}}^{\mathrm{miss}}\right)<60 \mathrm{GeV}$

In this $Z \rightarrow$ ee control region, the $e F F$ is defined as the ratio of identified to anti-identified numbers of events

$$
e F F=\frac{N_{\mathrm{data}, \mathrm{ZeeCR}}^{\mathrm{ID}}-N_{\mathrm{MC}, \text { not e } \rightarrow \tau, \text { ZeeCR }}^{\mathrm{ID}}}{N_{\mathrm{data}, \text { ZeeCR }}^{\text {anti-ID }}-N_{\mathrm{MC}, \text { not e } \rightarrow \tau, \text { ZeeCR }}^{\text {anti-ID }}}
$$

$e F F$ is estimated as a function of the $\tau_{\text {had-vis }} p_{\mathrm{T}}$, as shown in Figure 8.8.

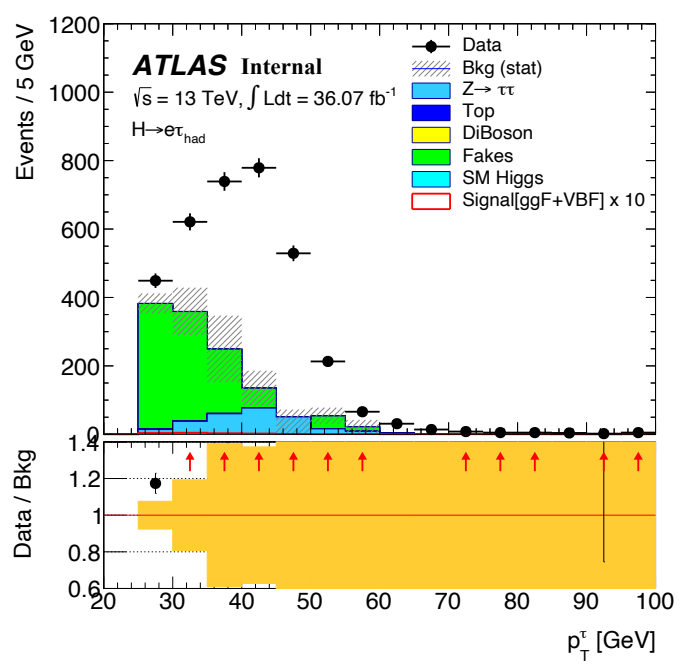

(a) ID region

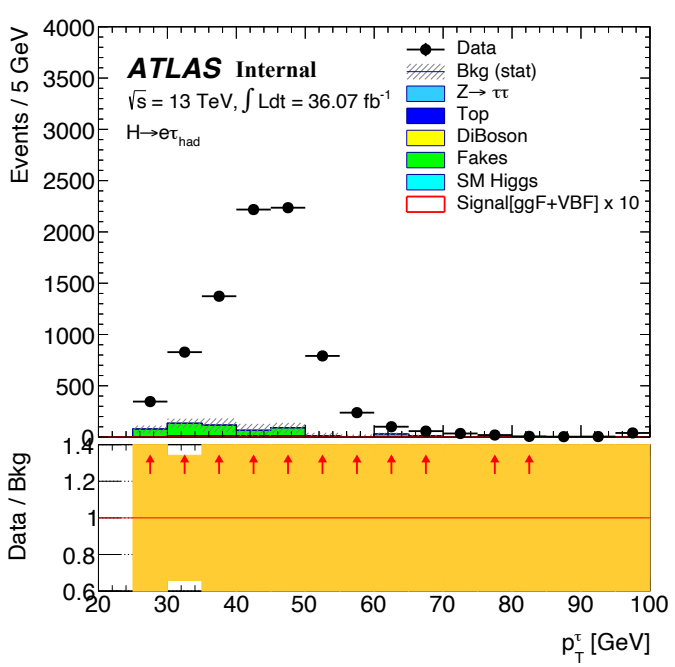

(b) anti-ID region

Figure 8.8: Distribution of $e \tau_{\text {had }}$ events as function of $\tau_{\text {had-vis }} p_{T}$ in the $Z \rightarrow e e$ (a) ID region (b) anti-ID region.

\subsubsection{Electron-faking-tau background estimation plots}

A set of plots in Figures 8.9, 8.10, and 8.11 show the comparison the $m_{\text {vis }}, m_{\text {coll }}$ and $p_{\mathrm{T}}(\tau)$ distributions using $\mathrm{MC}$ (top row) and data-driven (bottom row) $Z \rightarrow e e$ events in the $H \rightarrow e \tau_{\text {had }}$ channel for each of the CBA region labelled as SR1, SR2 and SR3, respectively. 

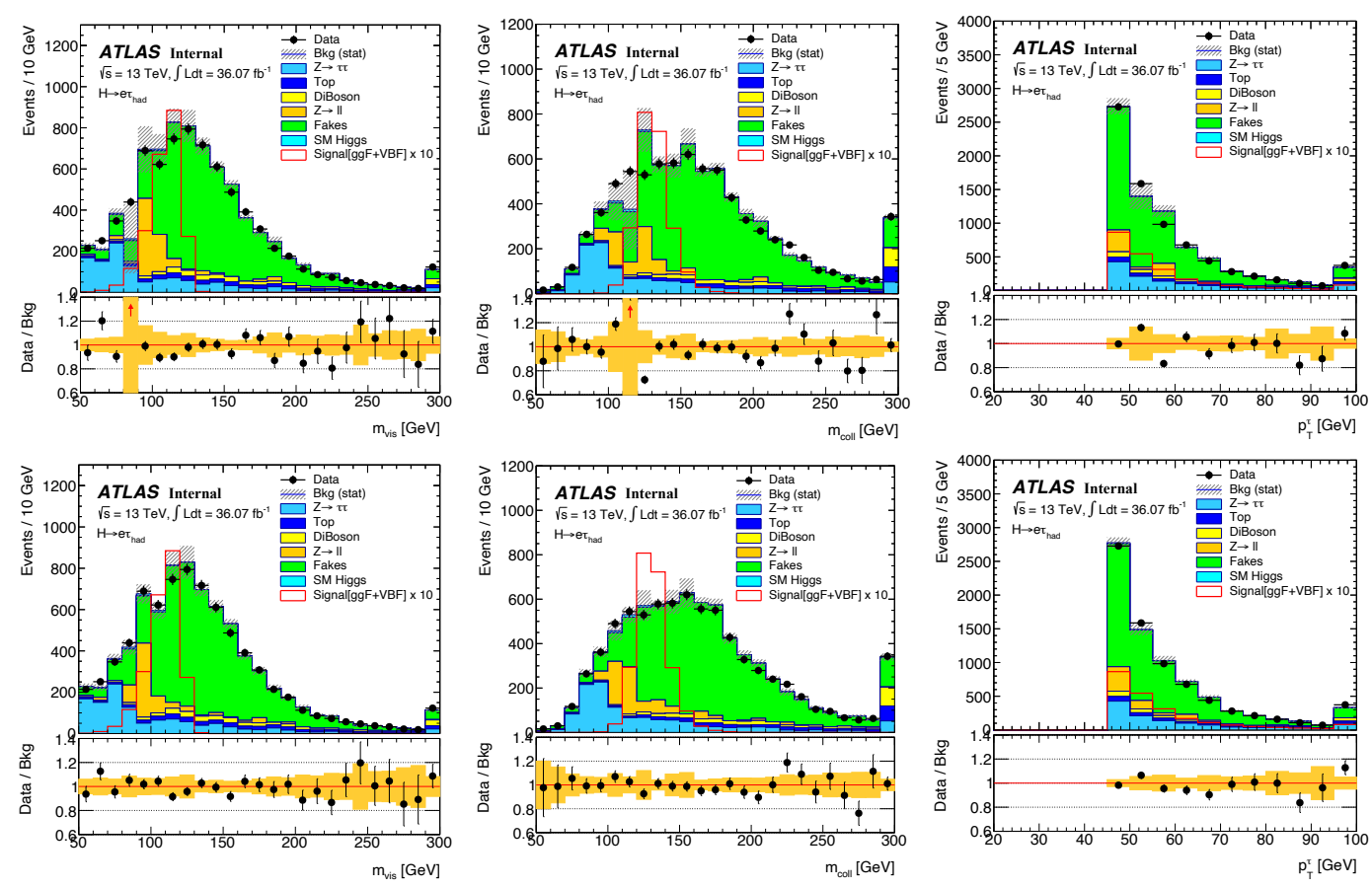

Figure 8.9: Comparison of the distributions of $m_{\mathrm{vis}}, m_{\text {coll }}$ and $p_{\mathrm{T}}(\tau)$ using MC (top row) and data-driven (bottom row) $Z \rightarrow e e$ events in the $H \rightarrow e \tau_{\text {had }}$ SR1 region. The statistical uncertainties for all samples and the weight and kinematic systematics for the background are shown. 

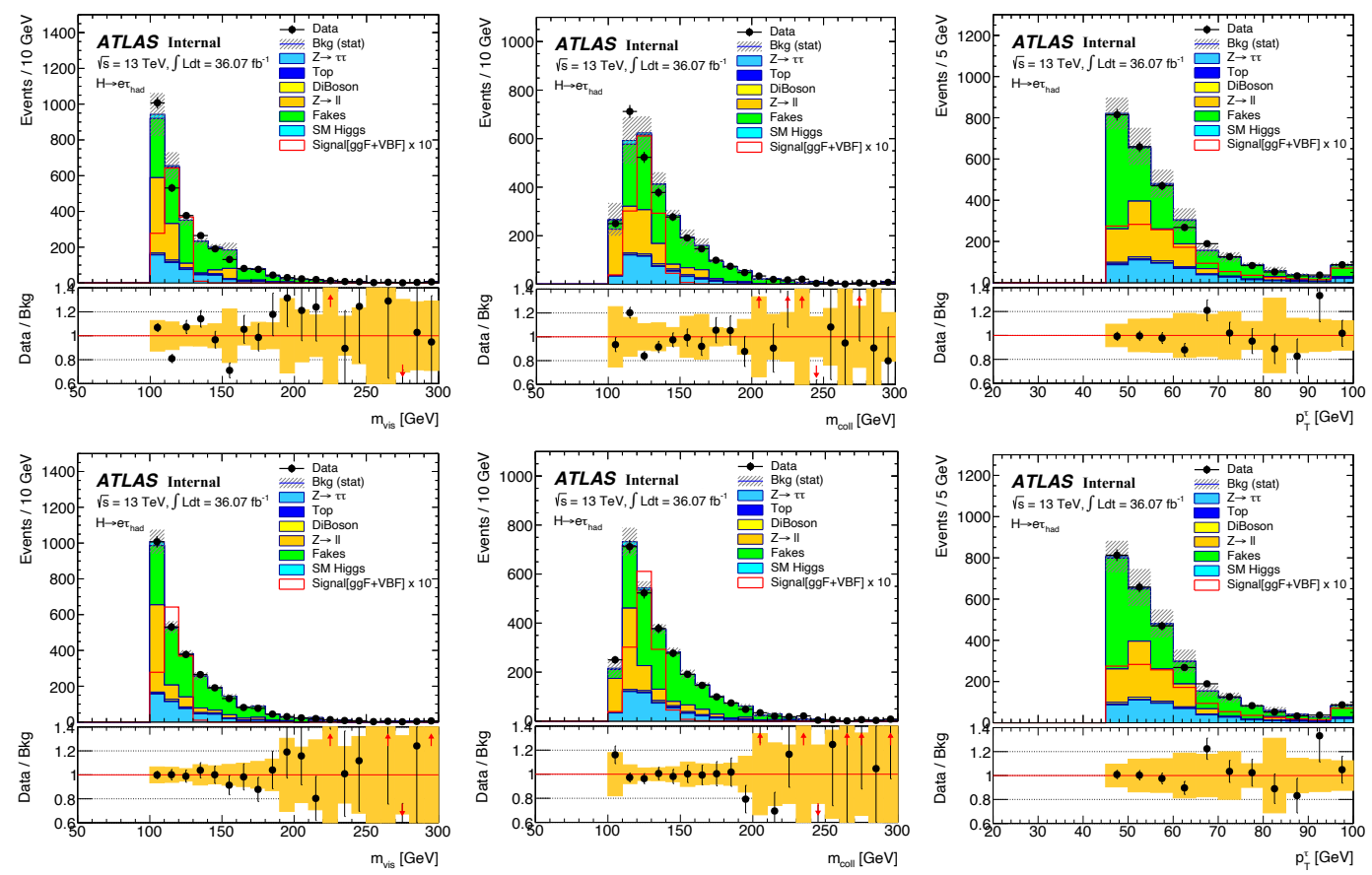

Figure 8.10: Comparison of the distributions of $m_{\mathrm{vis}}, m_{\text {coll }}$ and $p_{\mathrm{T}}(\tau)$ using $\mathrm{MC}$ (top row) and data-driven (bottom row) $Z \rightarrow e e$ events in the $H \rightarrow e \tau_{\text {had }}$ SR2 region. The statistical uncertainties for all samples and the weight and kinematic systematics for the background are shown. 

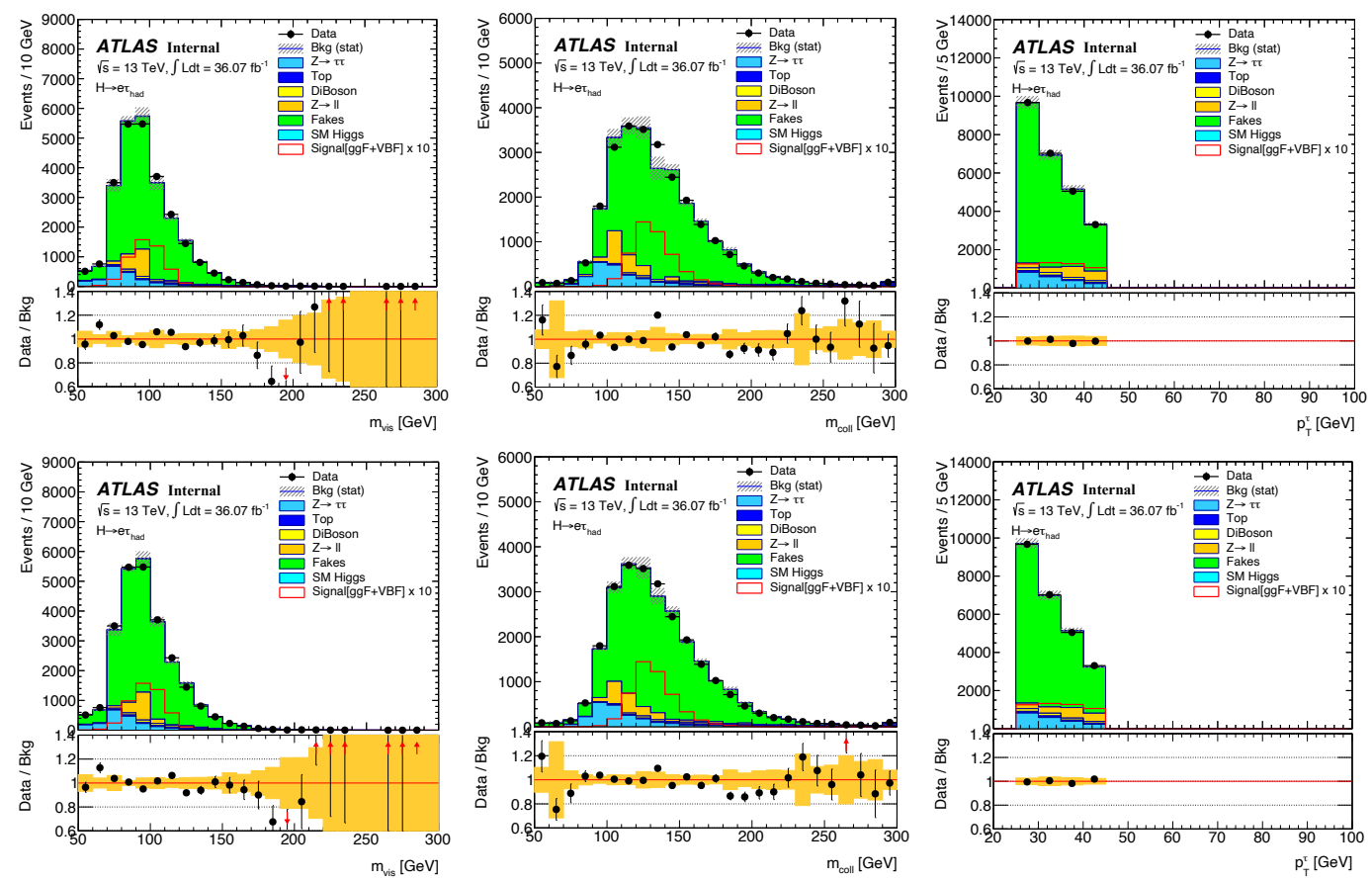

Figure 8.11: Comparison the distributions of $m_{\mathrm{vis}}, m_{\text {coll }}$ and $p_{\mathrm{T}}(\tau)$ using $\mathrm{MC}$ (top row) and data-driven (bottom row) $Z \rightarrow e e$ events in the $H \rightarrow e \tau_{\text {had }}$ SR3 region. The statistical uncertainties for all samples and the weight and kinematic systematics for the background are shown. 


\subsubsection{Closure tests of electron fakes}

In order to show that the electron-faking-tau background estimation by using the fake factor method performs as expected, Figure 8.12 shows the comparison between MC and data-driven $Z \rightarrow$ ee samples in the $Z \rightarrow e e$ control region with the same-sign requirement instead of the opposite-sign one. Here, the eFF derived opposite-sign (medium, not tight) region was used to estimate the data-driven $Z \rightarrow$ ee sample in the same sign region. The same sign region has a higher jet faking tau component, which reduces the purity of the region.

In addition, Figure 8.13 shows the comparison between $\mathrm{MC}$ and data-driven $Z \rightarrow$ ll samples in the opposite-sign (tight) $Z \rightarrow e e$ control region at preselection level with (medium, not tight) eFF requirement. The $Z \rightarrow e e$ purity in the oppositesign control region is better than for the same-sign region, especially for low $E_{\mathrm{T}}^{\text {miss }}$ values. The estimation of the $Z \rightarrow e e$ background obtained with the data-driven eFF method models the data better than the $Z \rightarrow e e \mathrm{MC}$ simulation.

\subsubsection{7 $Z \rightarrow \mu \mu$ validation plots}

In the case of the $Z \rightarrow \mu \mu$ background, it is not possible to define a fake factor in a way similar to the $e F F$. Additionally, the amount of $Z \rightarrow \ell \ell$ background in the $\mu \tau_{\text {had }}$ channel is significantly smaller than in the $e \tau_{\text {had }}$ channel. For these reasons, the $\mathrm{MC}$ simulation is used to model the $Z \rightarrow \mu \mu$ background in the $\mu \tau_{\text {had }}$ channel. Figure 8.14 shows the validation plots of the $Z \rightarrow \mu \mu$ background modelled by MC. The plots are shown in the $H \rightarrow \mu \tau_{\text {had }}$ channel. The event selection of the $Z \rightarrow \mu \mu$ control region are the same as the opposite-sign $Z \rightarrow$ ee control region, except for the leptons selection. There is a $\sim 10 \%$ difference in the bins dominated by $Z \rightarrow \mu \mu$ events, especially in the $m_{\text {coll }}$ distribution, which is accounted for as systematics in Section 8.6. 

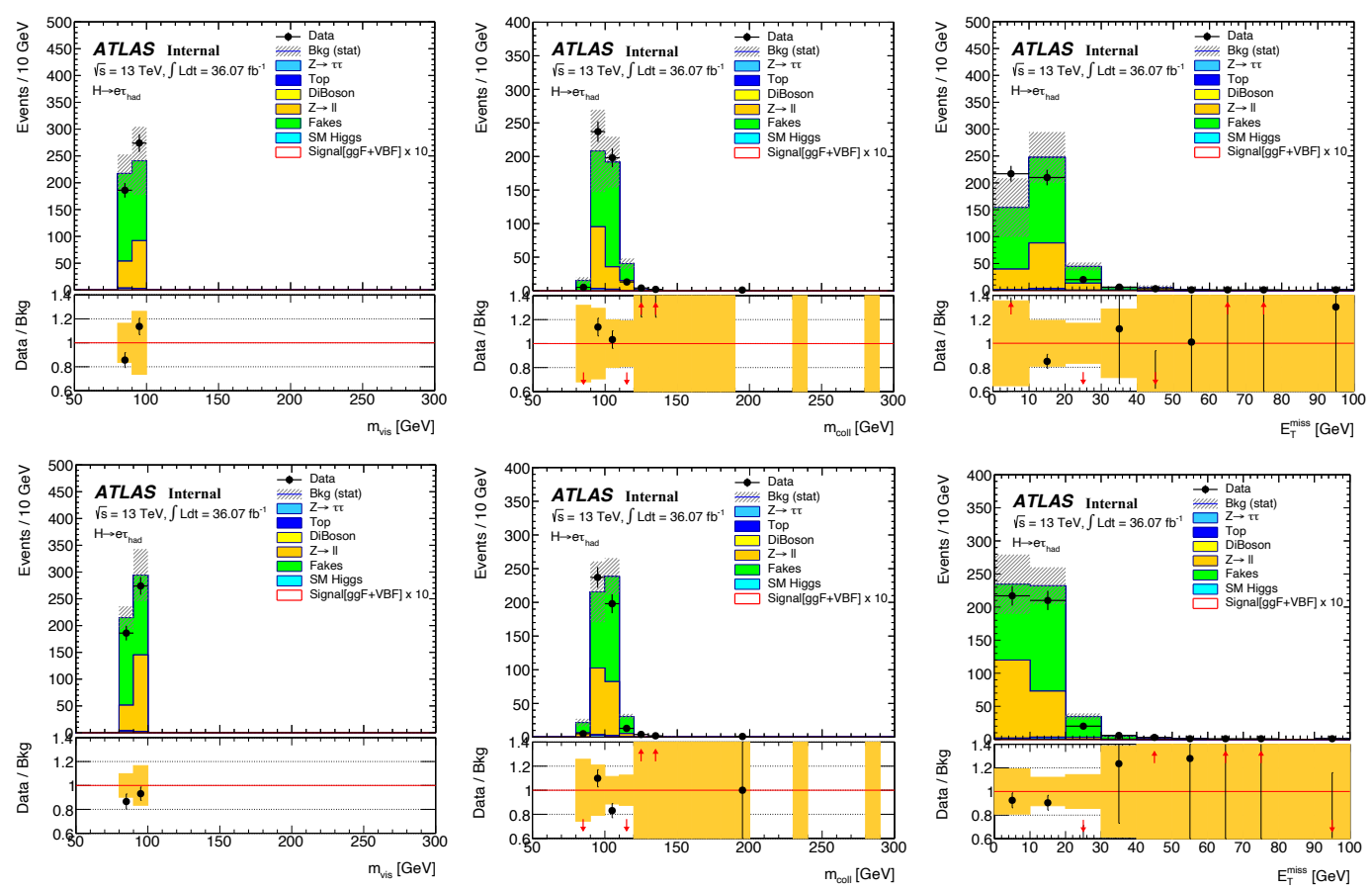

Figure 8.12: Comparison the distributions of $m_{\mathrm{vis}}, m_{\text {coll }}$ and $E_{\mathrm{T}}^{\mathrm{miss}}$ using $\mathrm{MC}$ (top row) and data-driven (bottom row) $Z \rightarrow e e$ events in the same-sign $Z \rightarrow e e$ control region. The hatched band in the upper plot, and the shaded band in the ratio plot, give the statistical only uncertainty. 

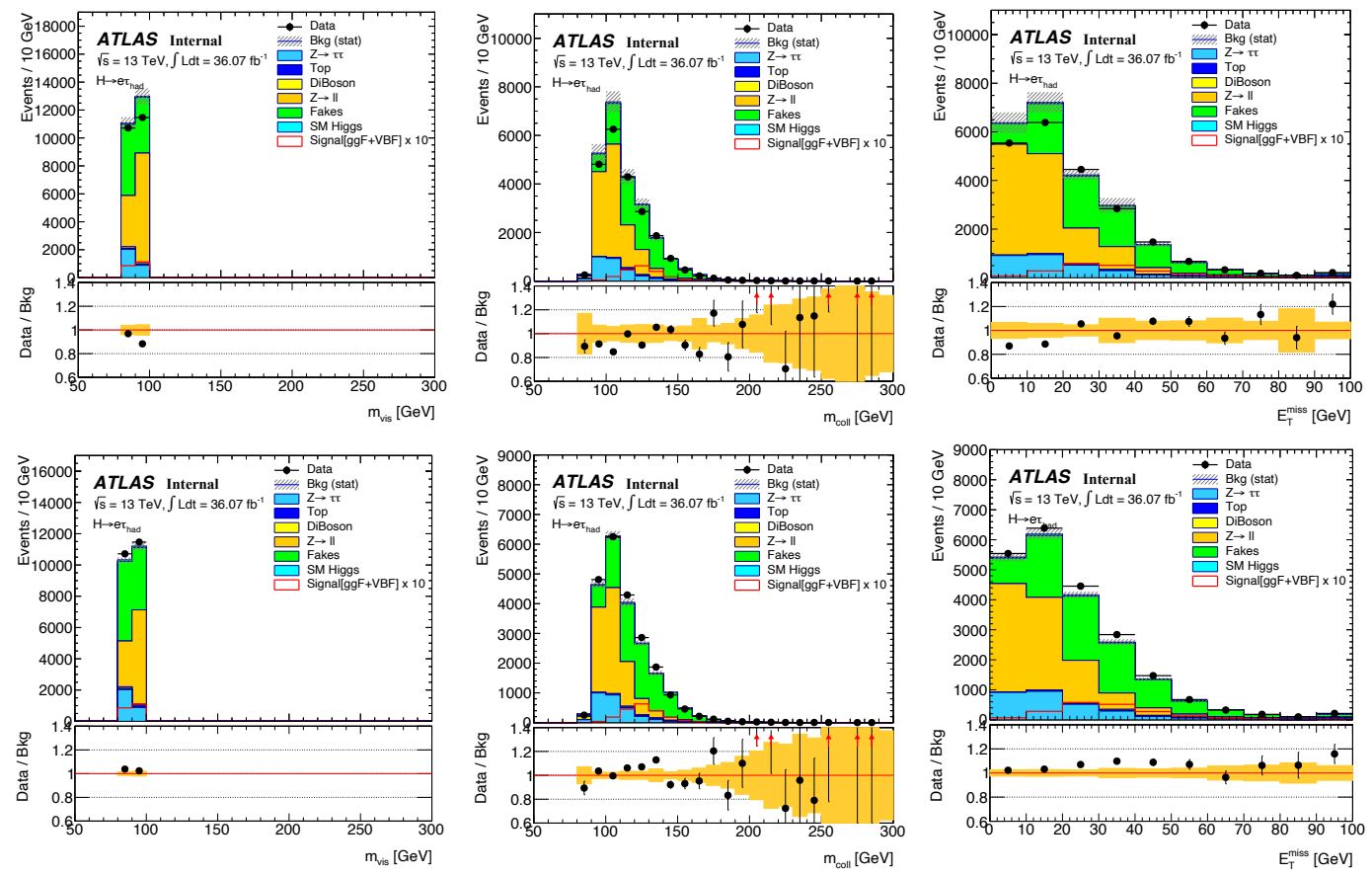

Figure 8.13: Comparison the distributions of $m_{\mathrm{vis}}, m_{\text {coll }}$ and $E_{\mathrm{T}}^{\text {miss }}$ using MC (top row) and data-driven (bottom row) $Z \rightarrow e e$ events in the opposite-sign $Z \rightarrow e e$ control region. The hatched band in the upper plot, and the shaded band in the ratio plot, give the statistical only uncertainty. 


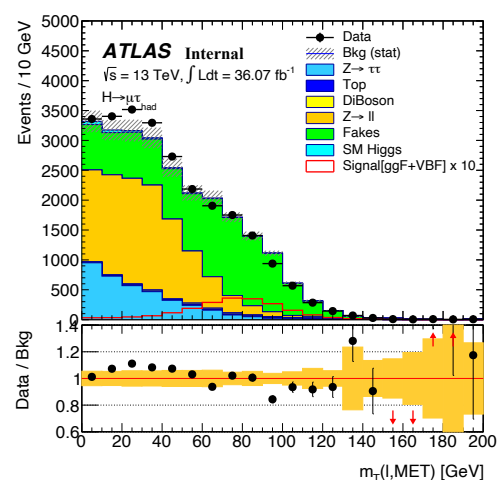

(a) $m_{\mathrm{T}}\left(\ell, E_{\mathrm{T}}^{\mathrm{miss}}\right)$

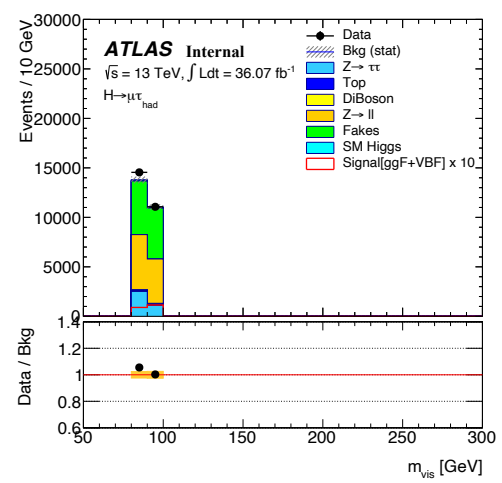

(d) $m_{\text {vis }}$

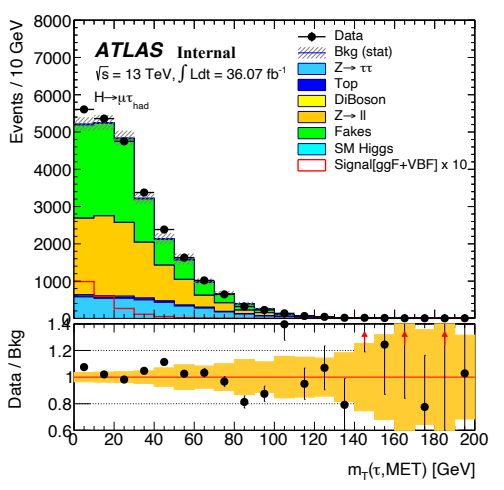

(b) $m_{\mathrm{T}}\left(\tau, E_{\mathrm{T}}^{\text {miss }}\right)$

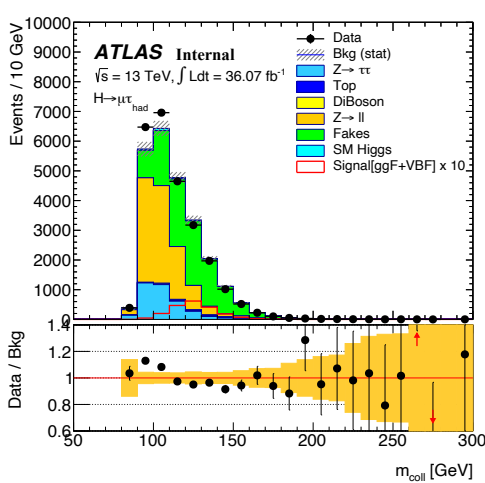

(e) $m_{\text {coll }}$

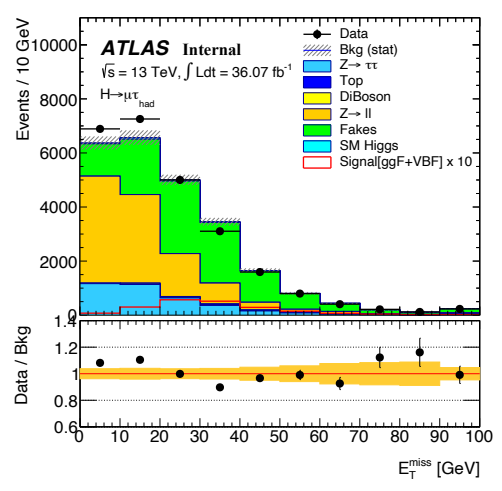

(c) $E_{\mathrm{T}}^{\mathrm{miss}}$

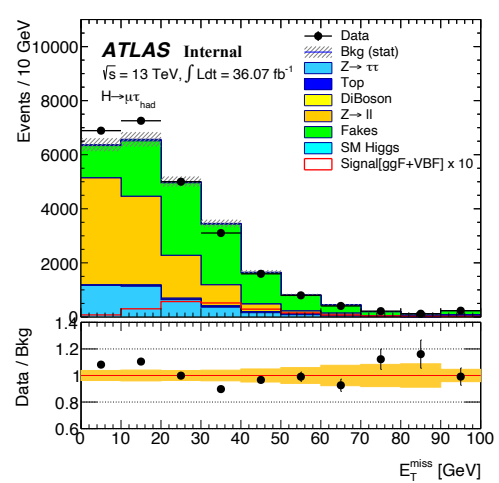

(f) $E_{\mathrm{T}}^{\text {miss }}$

Figure 8.14: Distributions of $\mu \tau_{\text {had }}$ events in the $Z \rightarrow \mu \mu$ control region. The hatched band in the upper plot, and the shaded band in the ratio plot, give the statistical only uncertainty. 


\subsection{Systematic uncertainties and pre-fit results}

The systematic uncertainties can affect the normalization of signal and background, and also the shape of their corresponding final discriminant distributions. Each source of systematic uncertainty is considered to be uncorrelated with the other sources. The size of the systematic uncertainties and their impact on the fitted branching ratio are discussed in Section 8.7. Envelope distributions with the magnitude of the systematics are shown in Section 8.6.2. The evaluation of the systematics follows the same strategy used in the $H \rightarrow \tau^{+} \tau^{-}$analysis and systematics are discribed in details in Reference [129].

\subsubsection{Sources of systematics}

The following sources of systematic uncertainties are associated with the detector simulation, the signal modelling and the data-driven background determination:

\subsubsection{Luminosity}

The integrated luminosity measurement has an uncertainty of $1.9 \%$ for the 2015 data and $2.2 \%$ for the 2016 data, and it is applied to all simulated event samples [130].

\subsubsection{Detector-related uncertainties}

Uncertainties related to the detector are included for the signal and backgrounds that are estimated using simulation. These uncertainties are also taken into account for simulated events that are used in the data-driven background estimations. All instrumental systematic uncertainties arising from the reconstruction, identification and energy scale of electrons, muons, ( $b$-)jets tagging, triggering efficiencies of all

physics objects and the soft term of the $E_{\mathrm{T}}^{\text {miss }}$ measurement are considered. The effect of the energy scale uncertainties on the objects is propagated to the $E_{\mathrm{T}}^{\text {miss }}$ 
determination.

These systematics include uncertainty associated with:

- the electron and muon trigger, reconstruction and identification efficiencies,

- jet, electron and muon energy scales,

- jet energy resolution and calibration,

- calibration of the $E_{\mathrm{T}}^{\mathrm{miss}}$,

- jet flavour tagging systematics.

Any systematic effect on the the overall normalisation or shape of the $m_{\text {coll }}$ distribution in the signal region is considered.

\subsubsection{Uncertainties on data-driven background estimations}

Systematic uncertainties resulting from the data-driven background estimation for fakes are described in Reference [129]. In the $\ell \tau_{\text {had }}$ channel, the systematic uncertainties are calculated in each of the Signal Region and include both the statistical uncertainty on the estimation and the closure test. Details of the methodology uncertainties can be found in Section 8.5.2.

The following systematic uncertainties are considered on the jet-fake factor method:

- The uncertainty on the individual fake factors, $F F_{\mathrm{QCD}}$ and $F F_{\mathrm{W}}$.

- The uncertainty on the estimation of $R_{\mathrm{QCD}}$ from data. This uncertainty arises from the measurement of the isolation factor: $I F$ statistical uncertainty; the difference between SSP and the nominal opposite-sign region; the contamination of the control region with events with true leptons which is subtracted using MC (to estimate this effect, the subtracted MC is varied by $\pm 35 \%$ ). 
- The statistical uncertainty (from both data and subtracted MC) in the anti-ID region where the fake factors are applied.

Table 8.10 and Table 8.11 show the effect of each of these systematic uncertainties on the fake estimate in each signal regions.

Table 8.10: Summary of the fake-estimate yields in the LFV $H \rightarrow \mu \tau_{\text {had }}$ signal regions, as well as the effect of each of the uncertainties discussed in the text with respect to the nominal yield.

\begin{tabular}{l|c|c|c|c|c|c|c|c}
\hline & \multicolumn{4}{|c|}{ Absolute yields } & \multicolumn{3}{c}{ Rel. diff. to nom. (\%) } \\
\hline & SR1 & SR2 & SR3 & VBF & SR1 & SR2 & SR3 & VBF \\
\hline Nominal & 3995.74 & 1050.33 & 17793.32 & 146.18 & \multicolumn{3}{|c}{} \\
\hline FF Stat. Unc. (up) & 4634.65 & 1225.80 & 19723.59 & 182.35 & 16 & 17 & 11 & 25 \\
\hline FF Stat. Unc. (down) & 3445.54 & 894.37 & 16290.49 & 110.02 & -16 & -17 & -11 & 25 \\
\hline$R_{\text {QCD }}$ (up) & 4116.35 & 1081.45 & 18503.67 & 154.95 & 3 & 3 & 4 & 6 \\
\hline$R_{\text {QCD }}$ (down) & 3899.32 & 1019.17 & 17105.34 & 137.41 & -3 & -3 & -4 & -6 \\
\hline Anti-ID Stat. (up) & 4235.44 & 1167.56 & 19038.16 & 156.42 & 6 & 11 & 7 & 7 \\
\hline Anti-ID Stat. (down) & 3769.64 & 945.71 & 16697.26 & 135.95 & -6 & -11 & -7 & -7 \\
\hline
\end{tabular}

Table 8.11: Summary of the fake-estimate yields in the LFV $H \rightarrow e \tau_{\text {had }}$ signal regions, as well as the effect of each of the uncertainties discussed in the text with respect to the nominal yield.

\begin{tabular}{l|c|c|c|c|c|c|c|c}
\hline & \multicolumn{5}{|c|}{ Absolute yields } & \multicolumn{3}{c}{ Rel. diff. to nom. (\%) } \\
\hline & SR1 & SR2 & SR3 & VBF & SR1 & SR2 & SR3 & VBF \\
\hline Nominal & 5189.99 & 1531.45 & 20734.33 & 177.81 & \multicolumn{3}{|c}{} \\
\hline FF Stat. Unc. (up) & 6074.95 & 1811.57 & 23015.63 & 231.62 & 17 & 18 & 11 & 30 \\
\hline FF Stat. Unc. (down) & 4438.29 & 1298.07 & 17689.23 & 124.01 & -17 & -18 & -10 & -30 \\
\hline$R_{\text {QCD }}$ (up) & 5397.04 & 1577.34 & 21777.48 & 188.48 & 4 & 3 & 5 & 6 \\
\hline$R_{\text {QCD }}$ (down) & 4991.34 & 1486.45 & 19674.93 & 167.14 & -4 & -3 & -6 & -6 \\
\hline Anti-ID Stat. (up) & 5439.50 & 1714.34 & 21978.29 & 190.26 & 5 & 12 & 6 & 7 \\
\hline Anti-ID Stat. (down) & 4994.87 & 1367.40 & 19854.27 & 165.37 & -4 & -12 & -6 & -7 \\
\hline
\end{tabular}

The following systematic uncertainties are considered on the $e \rightarrow \tau_{\text {had }}$ fake factor 
method:

- The uncertainty on the fake factors, $e F F$.

- The uncertainty due to the difference between tight and (medium, not tight) $e F F$. Figure 8.15 shows the comparisons of different fake factors $e F F$ derived in tight and (medium, not tight) $Z \rightarrow e e$ control regions. The difference between $e F F \mathrm{~s}$ in the left plot is taken as the systematic uncertainty.

- The contamination of the events with non electron-faking- $\tau_{\text {had }}$ in the anti-ID region, which are subtracted before applying eFF.

Table 8.12 shows the effect of each of these systematic uncertainties on the $e \rightarrow \tau_{\text {had }}$ estimate in each signal regions.

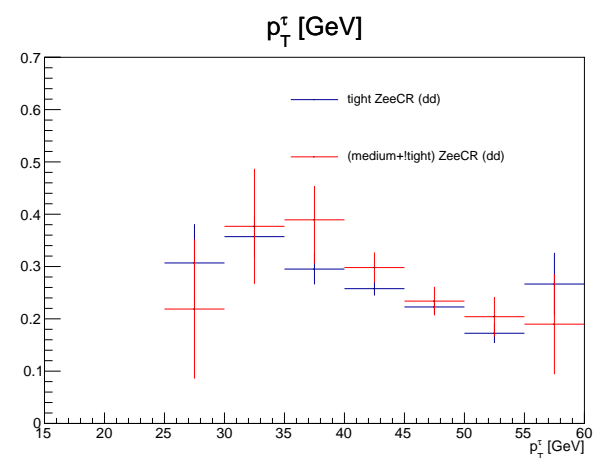

(a)

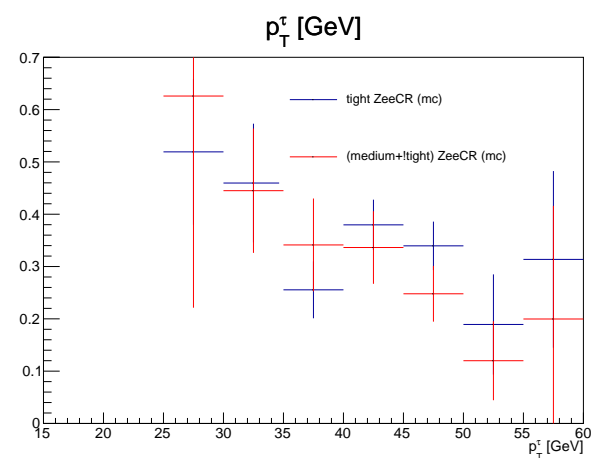

(b)

Figure 8.15: Comparison of $e F F$ derived in tight and (medium, not tight) $Z \rightarrow e e$ control regions (a) by fake factor method (b) by using $\mathrm{MC} Z \rightarrow e e$ events. 
Table 8.12: Summary of the $e \rightarrow \tau_{\text {had }}$ estimate yields in the LFV $H \rightarrow e \tau_{\text {had }}$ signal regions, as well as the effect of each of the uncertainties discussed in the text with respect to the nominal yield.

\begin{tabular}{l|c|c|c|c|c|c|c|c}
\hline & \multicolumn{4}{|c|}{ Absolute yields } & \multicolumn{3}{c}{ Rel. diff. to nom. (\%) } \\
\hline & SR1 & SR2 & SR3 & VBF & SR1 & SR2 & SR3 & VBF \\
\hline MC Zll & $612.86 \pm 210.77$ & $743.18 \pm 135.05$ & $1584.62 \pm 298.59$ & $52.39 \pm 28.16$ & & & & \\
Data-driven Zll (nom.) & $649.13 \pm 55.08$ & $575.19 \pm 32.87$ & $1598.44 \pm 96.88$ & $46.59 \pm 6.82$ & & & & \\
\hline FF Stat. Unc. (up) & $741.82 \pm 59.32$ & $655.16 \pm 39.56$ & $1986.12 \pm 115.43$ & $61.50 \pm 9.00$ & 14 & 14 & 23 & 32 \\
FF Stat. Unc. (down) & $564.91 \pm 48.01$ & $504.15 \pm 27.32$ & $1299.74 \pm 80.37$ & $31.68 \pm 4.63$ & 14 & 14 & 23 & 32 \\
\hline Tau ID & $762.34 \pm 61.32$ & $678.42 \pm 40.29$ & $1896.15 \pm 104.34$ & $54.51 \pm 7.97$ & 18 & 18 & 19 & 17 \\
\hline Bkg subtraction (up) & $704.18 \pm 58.31$ & $621.25 \pm 36.45$ & $1995.15 \pm 118.03$ & $55.44 \pm 8.11$ & 8 & 8 & 25 & 19 \\
Bkg subtraction (down) & $601.62 \pm 51.25$ & $533.58 \pm 28.99$ & $1278.25 \pm 79.04$ & $37.74 \pm 5.52$ & 8 & 8 & 25 & 19 \\
\hline
\end{tabular}

\subsubsection{Main backgrounds uncertainties}

Theoretical cross section uncertainties have been applied to the MC background samples used in this analysis. Uncertainties from missing higher-order corrections, the PDF parameterization, underlying-event modeling and from parton-shower modeling are also considered for the dominant $Z+$ jets background. Since its overall normalization is constrained separately in the VBF and nonVBF SRs, variations due to these uncertainties are considered in the event migration within an analysis channel, in the variable of interest shape and in the relative change in acceptance between the three analysis channels. These variations are treated as uncorrelated between the VBF and boosted SRs. In addition, the first two types of variations are treated as uncorrelated between the two analysis channels. This treatment accounts for the differences in the corresponding event selections. The largest sources of uncertainties are due to the CKKW matching and are evaluated depending on the number of true jets and the $Z$ boson $p_{\mathrm{T}}$.

For $Z+$ jets, generator parameter variations are estimated with Sherpa 2.1 samples. Both shape and acceptance variations of the following parameters are considered: ren, fac, qsf and ckkw, as well as parton shower uncertainties: 
- PDF: evaluated using event-weights provided by SHERPA.

- renormalisation and factorisation scales $-\mu_{R} / \mu_{F}$ : evaluated using eventweights provided by SHERPA.

- CKKW: jet-to-parton matching uncertainty, evaluated using truth-level parameterisation as a function of jet multiplicity and $\mathrm{p}_{\mathrm{T}}(\mathrm{Z})$.

- qsf: evaluated using truth-level parameterisation as a function of jet multiplicity and $\mathrm{p}_{\mathrm{T}}(\mathrm{Z})$.

- underlying-event/parton-shower: take MadGraph and SHERPAdifference using $Z \rightarrow \tau \tau$ events.

The uncertainty in the measured cross section for electroweak $Z$ production with two associated jets is found to be small compared to the other uncertainties in $Z$ boson production. All other simulated background contributions are normalized to their Monte Carlo prediction. For $Z+$ jets and diboson production, uncertainties of $5 \%$ and $6 \%$ are used, respectively, combining $\mathrm{PDF}+\alpha_{\mathrm{S}}$ and scale variation uncertainties in quadrature.

For $t \bar{t}[131]$ and single top quark $[132,133]$ production, a $6 \%$ uncertainty is assigned as total uncertainty based on scale, PDF and top-quark mass uncertainties.

Additional uncertainties include initial- and final-state radiation modelling, tune and (for $t \bar{t}$ only) the choice of the hdamp parameter value in POWHEG-BOX v2, which controls the amount of radiation produced by the parton shower [134]. The uncertainty on the fragmentation model is evaluated by comparing $t \bar{t}$ events generated with POWHEG-BOX v2 interfaced to either HERWIG++ [135] or PYTHIA6. The POWHEG+HERWIG++ and aMC@NLO+HERWIG++ generators are compared to estimate the uncertainty in generating the hard scatter. 


\subsubsection{Uncertainties on Higgs production modelling}

The procedures to estimate the uncertainty in the Higgs production cross sections follow the recommendations by the LHC Higgs Cross Section Working Group. Uncertainties are evaluated separately for their impact on the total cross section, their impact on the acceptance in different SRs, and on the shape of the variable of interest distribution in each SR. For the Higgs samples, uncertainties from the factorization and renormalization scale choices are estimated from the effect on the signal acceptance of doubling or halving these factors either coherently or oppositely. Uncertainties due to the initial- and final-state radiation, as well as multiple parton interaction for the signal, are also taken into account. These uncertainties are es-

timated from the AZNLO PYTHIA8 tune [136] for the gluon-gluon fusion and VBF production. The envelope of the variations resulting from the use of the alternative PDFs in the PDF4LHC15_nlo_100 [97] set is used in order to estimate the PDF uncertainty for gluon-gluon fusion production.

\subsubsection{Pre-fit results}

Good modelling of the background is demonstrated for a set of important BDT input variables in Figure 8.16, and the $m_{\text {coll }}$ distributions for CBA in Figures 8.17 and 8.18. 

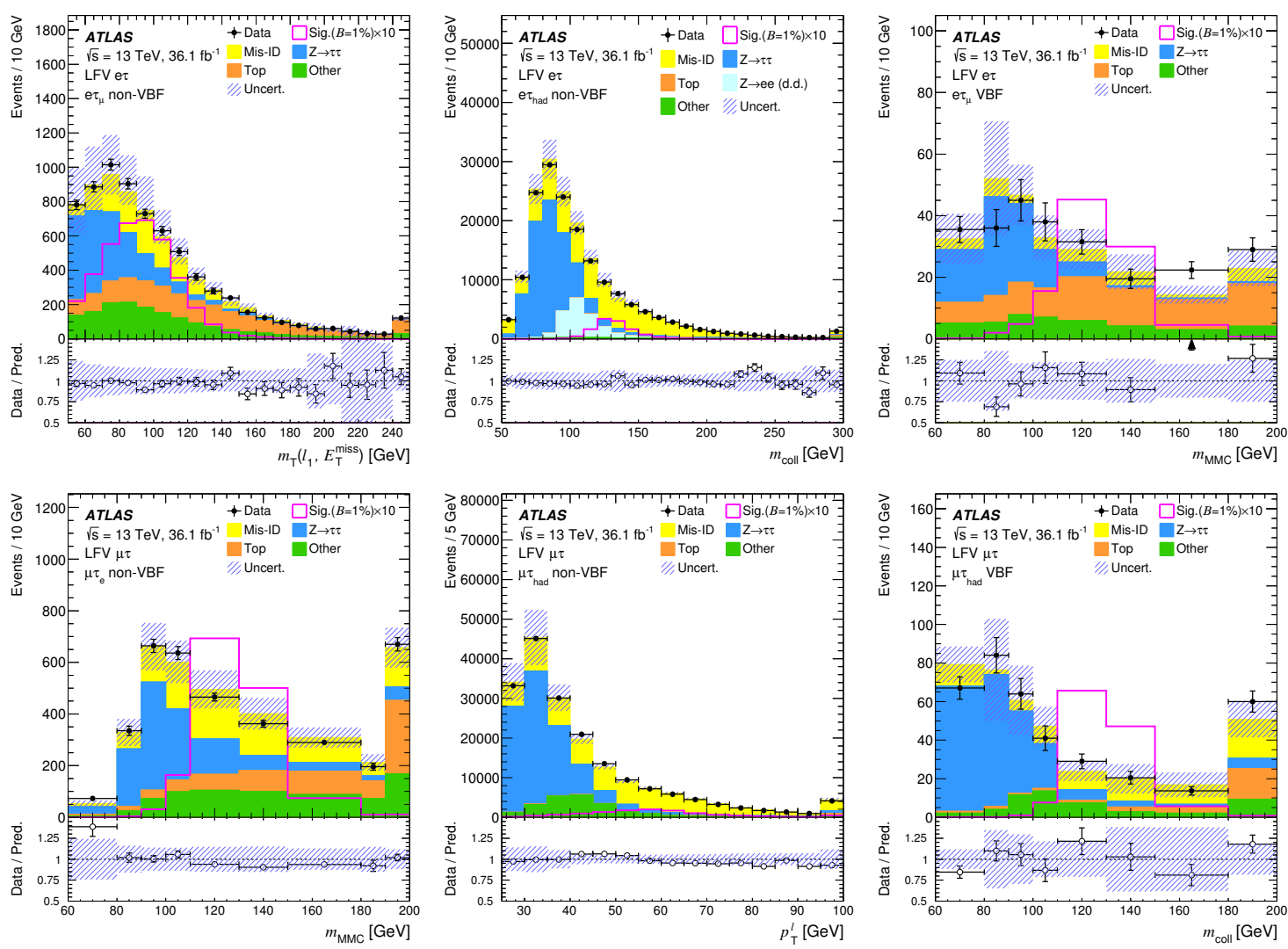

Figure 8.16: Distributions of representative kinematic quantities for different searches, channels and categories, before the fit as described in Section 8.7 is applied. Top row: transverse mass $m_{\mathrm{T}}\left(\ell_{1}, E_{\mathrm{T}}^{\text {miss }}\right)\left(e \tau_{\mu}\right.$ non-VBF), collinear mass $m_{\text {coll }}\left(e \tau_{\text {had }}\right.$ non$\mathrm{VBF})$ and $m_{\mathrm{MMC}}\left(e \tau_{\mu} \mathrm{VBF}\right)$. Bottom row: $m_{\mathrm{MMC}}\left(\mu \tau_{e}\right.$ non-VBF $)$, muon $p_{\mathrm{T}}\left(\mu \tau_{\text {had }}\right.$ non-VBF) and $m_{\text {coll }}\left(\mu \tau_{\text {had }} \mathrm{VBF}\right)$. Entries with values that would exceed the $x$-axis range are included in the last bin of each distribution. The size of the combined statistical, experimental and theoretical uncertainties in the background is indicated by the hatched bands. The $H \rightarrow e \tau(H \rightarrow \mu \tau)$ signal overlaid in top (bottom) plots assumes $\mathcal{B}(H \rightarrow \ell \tau)=1 \%$ and is enhanced by a factor 10 . In the data/background prediction ratio plots, points outside the displayed $y$-axis range are shown by arrows. 

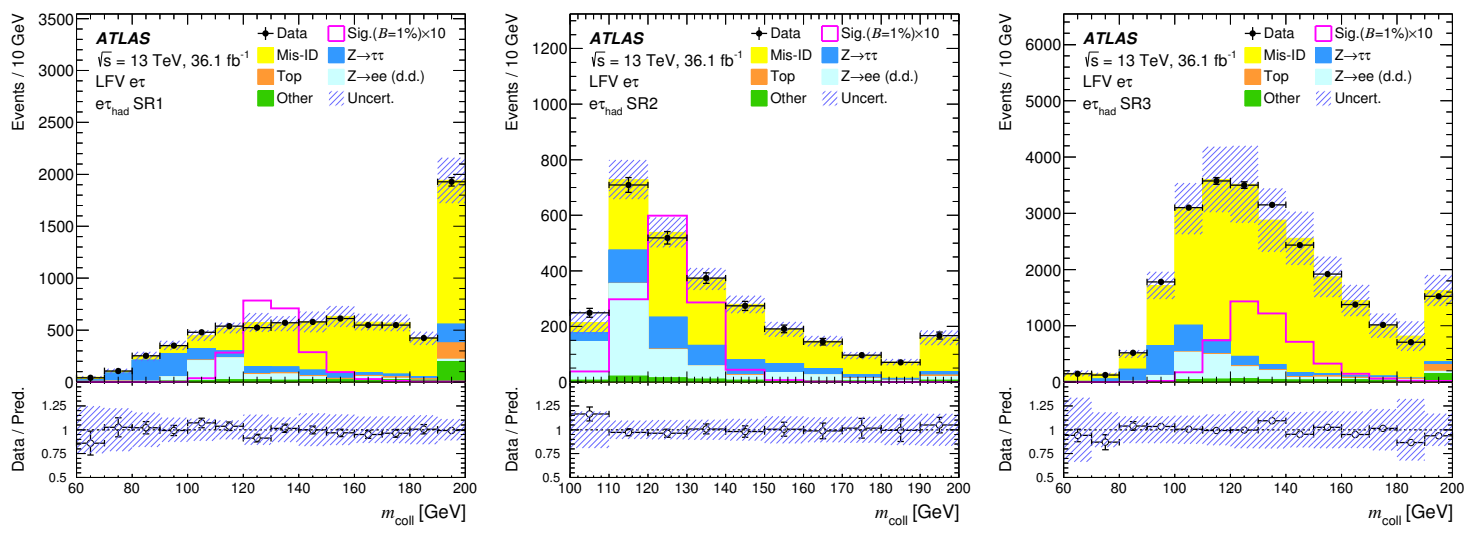

Figure 8.17: Pre-fit distributions of the Higgs boson mass for the $e \tau_{\text {had }}$ SR1-SR3 of the cut-based analysis. Entries with values that would exceed the $x$-axis range are included in the last bin of each distribution. The size of the combined statistical, experimental and theoretical uncertainties in the background is indicated by the hatched bands. The $H \rightarrow e \tau$ signal with $\mathcal{B}(H \rightarrow e \tau)=1 \%$ is overlaid and enhanced by a factor 10 . The binning is shown as in the statistical analysis.
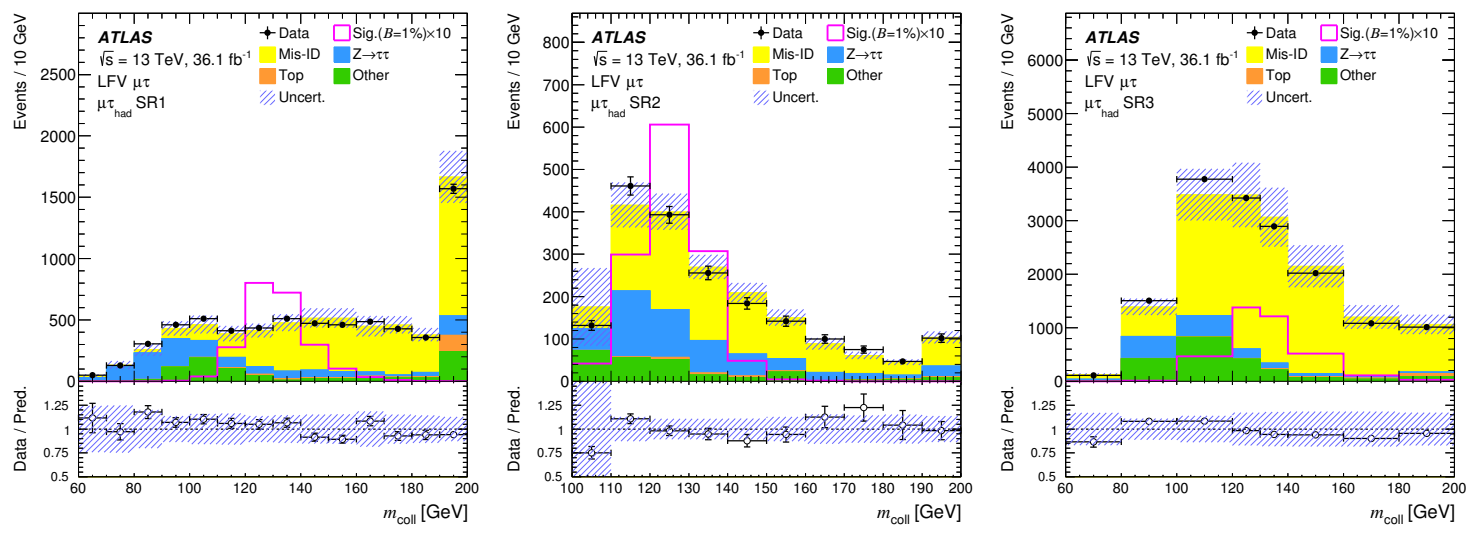

Figure 8.18: Pre-fit distributions of the Higgs boson mass for the $\mu \tau_{\text {had }}$ SR1-SR3 of the cut-based analysis. Entries with values that would exceed the $x$-axis range are shown in the last bin of each distribution. The size of the combined statistical, experimental and theoretical uncertainties in the background is indicated by the hatched bands. The $H \rightarrow \mu \tau$ signal with $\mathcal{B}(H \rightarrow \mu \tau)=1 \%$ is overlaid and enhanced by a factor 10 . The binning is shown as in the statistical analysis. 


\subsection{Statistical analysis}

The parameter of interest in the two searches of this thesis is the branching fraction of the lepton-flavour-violating decays of the Higgs boson in the $\mu \tau_{\text {had }}$ and $e \tau_{\text {had }}$ final state respetively. The statistical analysis uses a binned likelihood function $\mathcal{L}(\mu, \theta)$, constructed as a product of Poisson probability terms over all bins considered in the search. This function depends on the parameter $\mu$, defined as the branching ratio $\operatorname{BR}\left(\ell \tau_{\text {had }}\right)$ in $\%$, and a set of nuisance parameters $\theta$ that encode the effect of systematic uncertainties in the signal and background expectations.

The fitted central values and errors of the nuisance parameters (NP) are expected to follow a normal distribution centered around 0 with unit width, if the Asimov data is used. The fit model construction is obtained with the Roofit [137, 138] and RooStats [137, 138] software, and the model configuration file (as input to RooStats) is produced by WorkspaceBuilder [139], which is a software package interface with HistFactory [139]. WorkspaceBuilder includes additional features such as histogram smoothing, NP pruning (NPs whose impact are less than a certain threshold are discarded) and error symmetrization before the fits. A procedure called local symmetrization to the systematic variational histograms is implemented to symmetrize bins with one-sided variations which may cause problems in the fit. The bin variation is set to the maximum of the up and down variations, the larger variation determining its sign. The bin-by-bin fluctuations in the combined MC templates are also treated as NPs. They are incorporated in the model as extra Poisson constraint terms, and are expected to have a fitted value of 1 and a fitted error reflecting the relative statistical error in each particular bin.

The best-fit $\mathrm{BR}\left(\ell \tau_{\text {had }}\right)$ is obtained by performing a binned likelihood fit to the data under the signal-plus-background hypothesis, i.e. maximizing $\mathcal{L}(\mu, \theta)$ over $\mu$ and $\theta$.

The test statistic $q_{\mu}$ is defined as the profile likelihood ratio, $q_{\mu}=-2 \ln [\mathcal{L}(\mu, \hat{\hat{\theta}}) / \mathcal{L}(\hat{\mu}, \hat{\theta})]$, 
where $\hat{\mu}$ and $\hat{\theta}$ maximize $\mathcal{L}$, and $\hat{\hat{\theta}}$ is the set of values that maximizes $\mathcal{L}$ for a given value of $\mu$.

For the discovery hypothesis, the test statistic is defined by setting $\mu=0$ in the profile likelihood ratio. In the absence of any significant excess above the background expectation, upper limits on $\mathrm{BR}\left(\ell \tau_{\text {had }}\right)$ are derived by using $q_{\mu}$ and the $\mathrm{CL}_{s}$ method [140].

There are four parameters freely floating in the fit without any constraints, namely, the signal strength $\mu$ (SigXsecOverSM), the top background normalization factor (norm_top), and the $Z \rightarrow \tau \tau$ background normalization factor for the inclusive (norm_inc_ztt) and VBF regions (norm_vbf_ztt). The last three are applied on the top and $Z \rightarrow \tau \tau$ events in the top and $Z \rightarrow \tau \tau$ CRs respectively, as well as in the $\mathrm{SR}$, to determine their rates in the $\mathrm{SR}$ in a data driven way. The errors associated with the different systematics, and the error associated with the top estimation using top CR, will be properly propagated to the fitted error of $\mu$ in a simultaneous fit of multiple regions via a profiled likelihood scan by the minimization program MINUIT.

This section describes the fit of the Nuisance Parameters of the $e \tau$ and $\mu \tau$ search for the MVA analysis. Figure 8.19 and Figure 8.20 shows the ranking of the Nuisance Parameters according to their impact on $\mu$. Fakes are the highest contributor for $e \tau$ and $\mu \tau$ respectively. No big pulls are observed, while fake Nuisance parameters are rather constrained, due to the large fake statistics.

Table 8.13 shows a summary of the uncertainties on $\mu(H \rightarrow \ell \tau)$, listing their impact on the signal hypothesis of $\operatorname{BR}(H \rightarrow \ell \tau)=1 \%$. The uncertainties associated with mis-identified leptons/jets and those related to the jet energy scale and resolution exhibit the highest impact on the measured branching ratio in both searches.

A very important conclusion from this Table 8.13 is that the precision of this analysis is not limited by size of the dataset analyzed, but the systematic variations are the dominant component of the total uncertainties. 


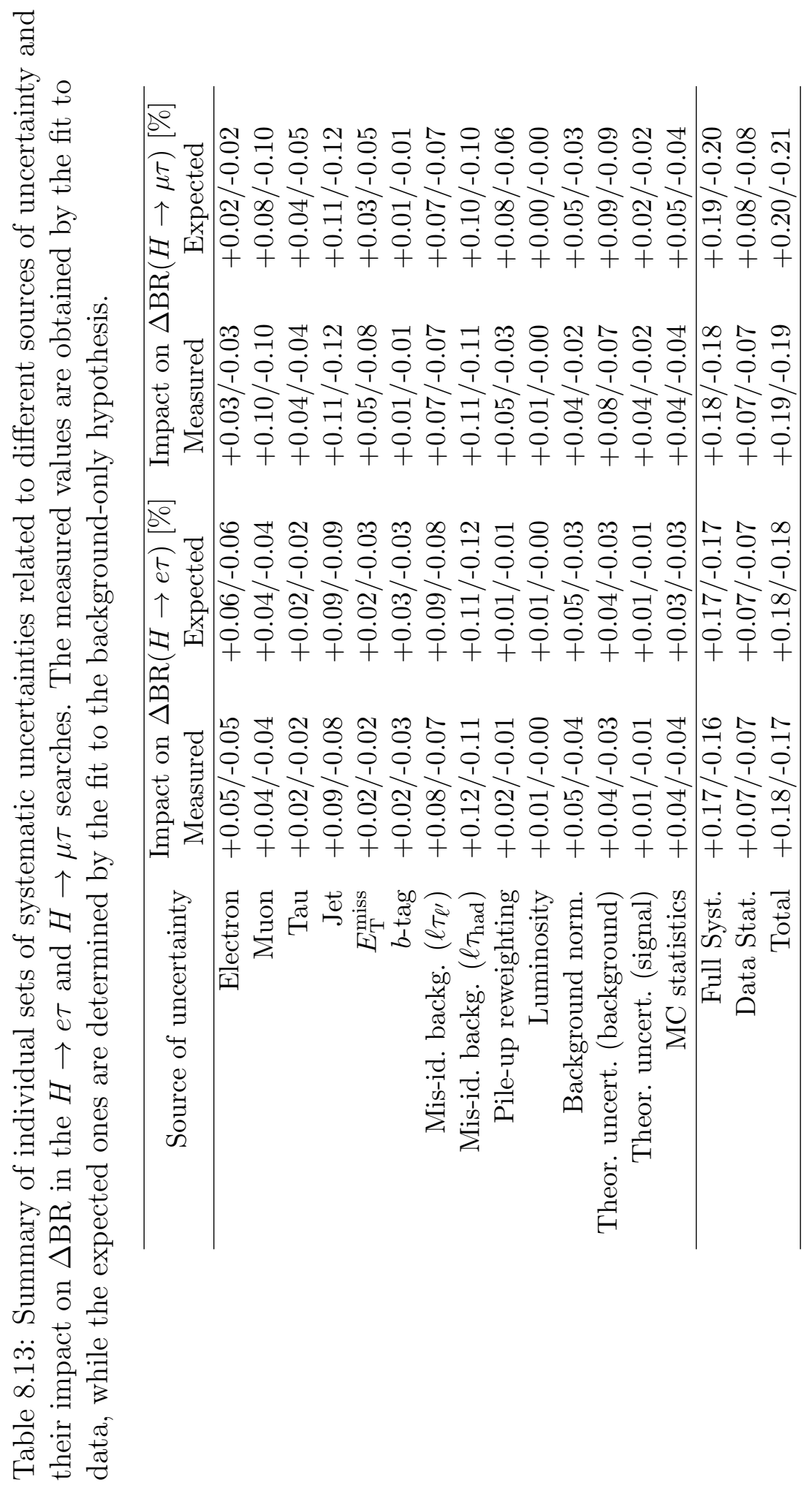




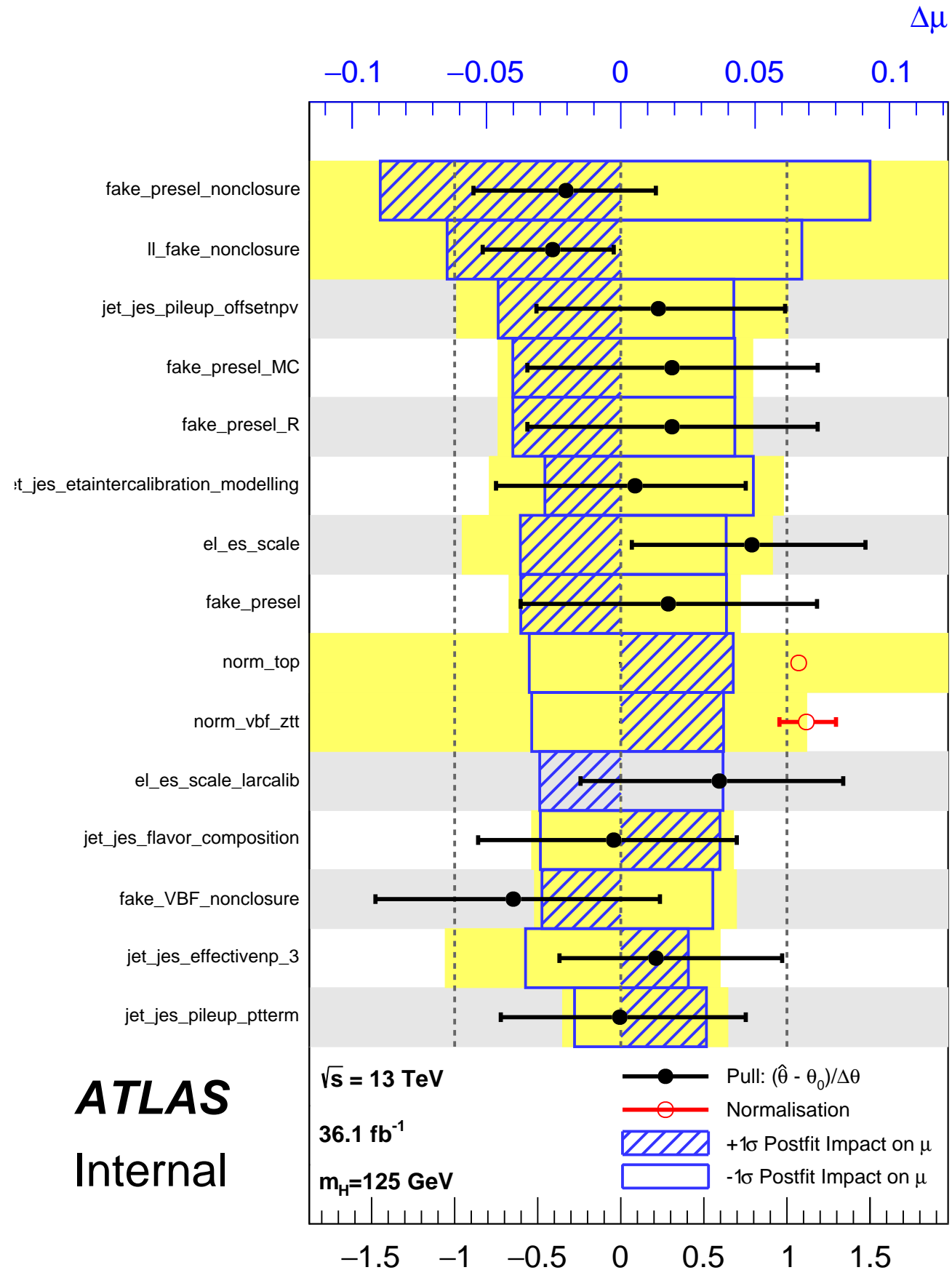

Figure 8.19: The S+B fit ranking of different NPs in the combined MVA e $\tau$ channel. The scale of the relative impact on $\mu$ (the NP pull) of the NPs is shown on the top (bottom) axis. 


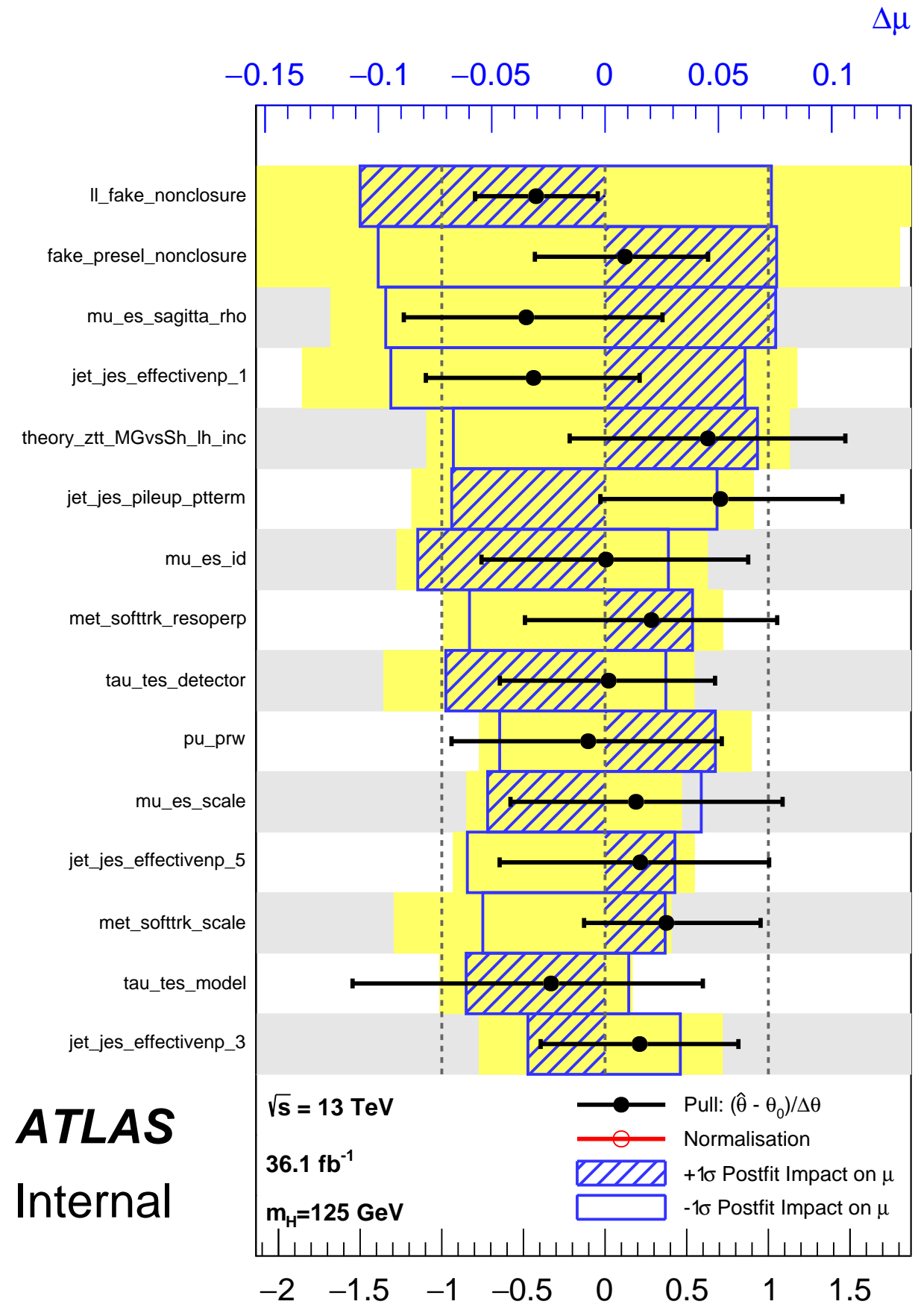

Figure 8.20: The S+B fit ranking of different NPs in the combined MVA $\mu \tau$ channel. The scale of the relative impact on $\mu$ (the NP pull) of the NPs is shown on the top (bottom) axis. 


\subsection{Results and combined sensitivity}

The discriminant distributions after the fit in each channel are shown in Figures 8.21 and 8.22. Good agreement between data and the background expectation is observed.
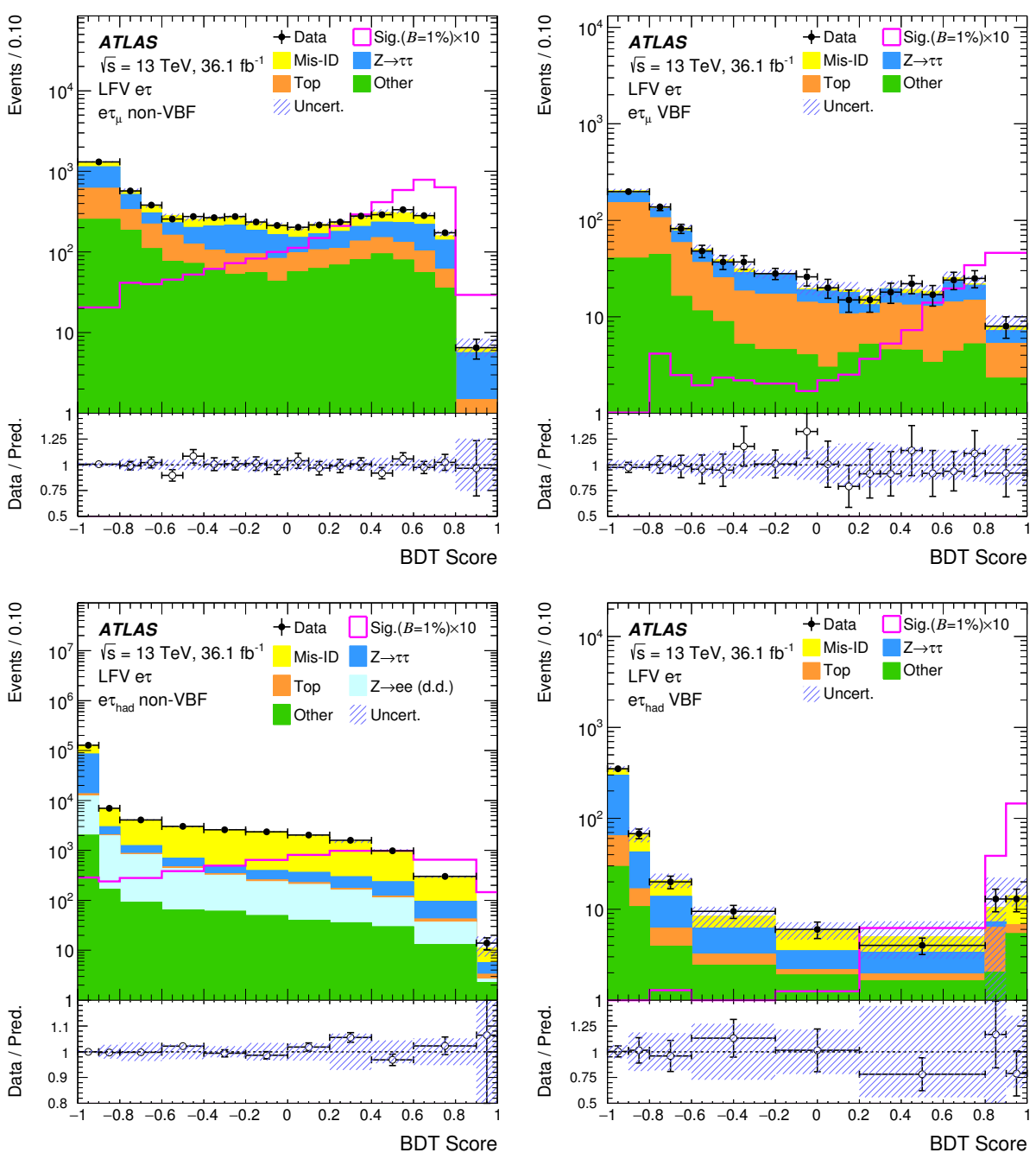

Figure 8.21: Distributions of the BDT score after the background+signal fit in each signal region of the $e \tau$ search, with the LFV signal overlaid, normalized with $\mathcal{B}(H \rightarrow$ $e \tau)=1 \%$ and enhanced by a factor 10 for visibility. The top and bottom plots display $e \tau_{\mu}$ and $e \tau_{\text {had }}$ BDT scores respectively, the left (right) column corresponds to the non-VBF (VBF) category. The size of the combined statistical, experimental and theoretical uncertainties of the background is indicated by the hatched bands. The binning is shown as in the statistical analysis. 

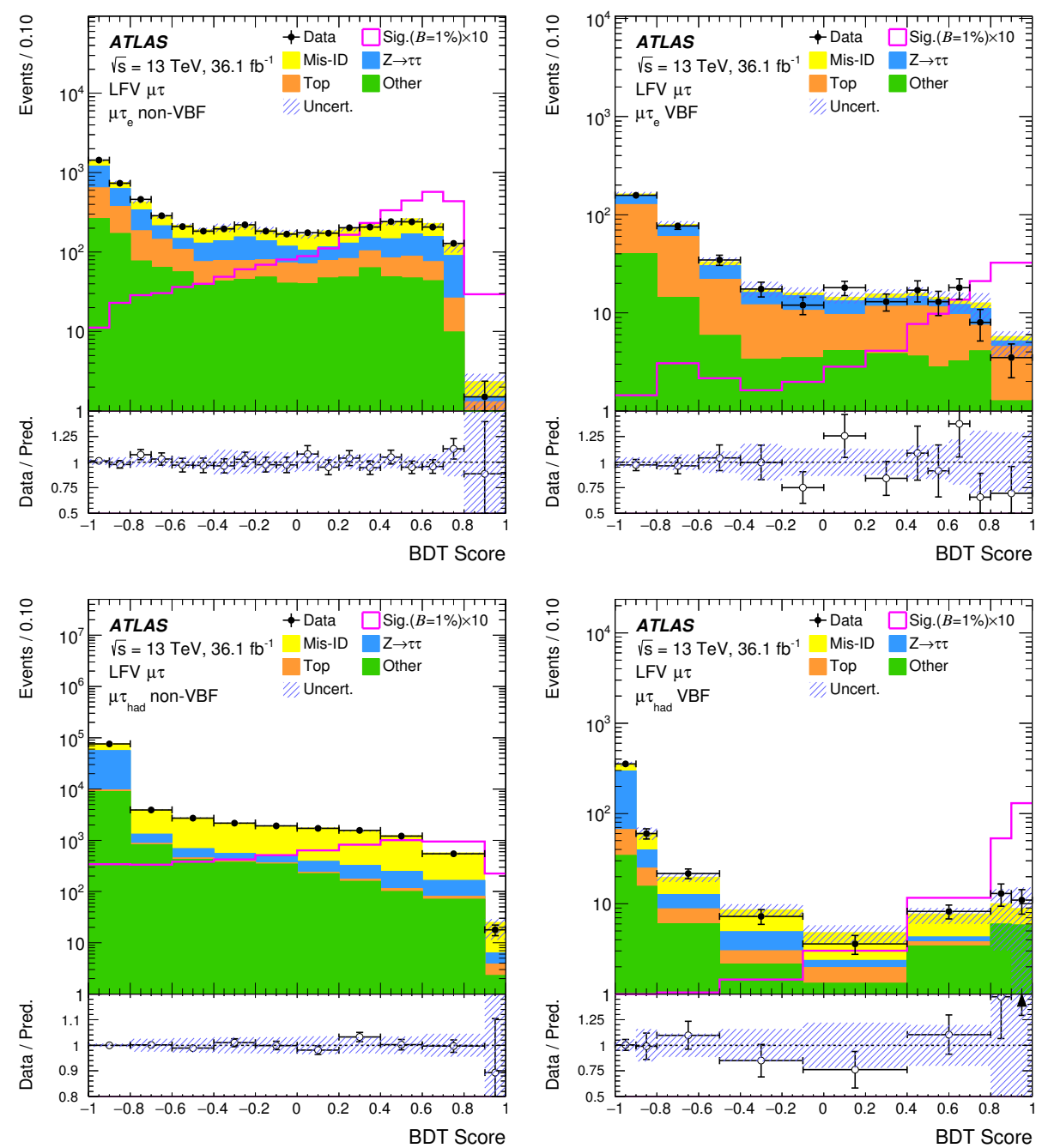

Figure 8.22: Distributions of the BDT score after the background+signal fit in each signal region of the $\mu \tau$ search, with the LFV signal overlaid, normalized with $\mathcal{B}(H \rightarrow$ $\mu \tau)=1 \%$ and enhanced by a factor 10 for visibility. The top and bottom plots display $\mu \tau_{e}$ and $\mu \tau_{\text {had }}$ BDT scores respectively, the left (right) column corresponds to the non-VBF (VBF) category. The size of the combined statistical, experimental and theoretical uncertainties of the background is indicated by the hatched bands. The binning is shown as in the statistical analysis. In the data/background prediction ratio plots, points outside the displayed $y$-axis range are shown by arrows. 
The individual contributions are listed in Tables 8.14 and 8.15, where the expected signal event yields are given for $\mathcal{B}(H \rightarrow \ell \tau)=1 \%$ and the rest obtained from background-only fit.

Table 8.14: Event yields and predictions as determined by the background-only fit in different signal regions of the $H \rightarrow e \tau$ analysis. Uncertainties include both the statistical a nd systematic contributions. "Other" contains diboson, $Z \rightarrow \ell \ell, H \rightarrow \tau \tau$ and $H \rightarrow W W$ background processes. For the $e \tau_{\text {had }}$ channel the " $Z \rightarrow e e$ (d.d.)" component corresponds to electrons misidentified as $\tau_{\text {had-vis }}$. This contribution is summed with "Other" since there are few events in the VBF category. The uncertainty of the total background includes all correlations between channels. The normalizations of top-quark ( $\ell \tau_{\ell^{\prime}}$ channel only) and $Z \rightarrow \tau \tau$ background components are determined by the fit.

\begin{tabular}{lcccc}
\hline & $e \tau_{\mu}$ non-VBF & $e \tau_{\mu}$ VBF & $e \tau_{\text {had }}$ non-VBF & $e \tau_{\text {had }}$ VBF \\
\hline Signal & $379 \pm 31$ & $19.8 \pm 2.7$ & $1180 \pm 110$ & $25 \pm 4$ \\
\hline$Z \rightarrow \tau \tau$ & $2470 \pm 230$ & $221 \pm 34$ & $73800 \pm 1900$ & $290 \pm 40$ \\
Top-quark & $1640 \pm 140$ & $490 \pm 40$ & $1580 \pm 190$ & $56 \pm 12$ \\
Mis-identified & $1330 \pm 250$ & $73 \pm 33$ & $74400 \pm 1600$ & $140 \pm 50$ \\
$Z \rightarrow e e$ (d.d.) & & & $15900 \pm 1800$ & $82 \pm 13$ \\
Other & $1700 \pm 80$ & $220 \pm 15$ & $2960 \pm 200$ & \\
\hline Total background & $7130 \pm 100$ & $1003 \pm 33$ & $168700 \pm 1000$ & $570 \pm 40$ \\
Data & 7128 & 992 & 168883 & 572 \\
\hline
\end{tabular}

Table 8.15: Event yields and predictions as determined by the background-only fit in different signal regions of the $H \rightarrow \mu \tau$ analysis. Uncertainties include both the statistical and systematic contributions. "Other" contains diboson, $Z \rightarrow \ell \ell, H \rightarrow \tau \tau$ and $H \rightarrow W W$ background processes. The uncertainty of the total background includes all correlations between channels. The normalizations of top-quark $\left(\ell \tau_{\ell^{\prime}}\right.$ channel only) and $Z \rightarrow \tau \tau$ background components are determined by the fit.

\begin{tabular}{lcccc}
\hline & $\mu \tau_{e}$ non-VBF & $\mu \tau_{e}$ VBF & $\mu \tau_{\text {had }}$ non-VBF & $\mu \tau_{\text {had }}$ VBF \\
\hline Signal & $287 \pm 23$ & $14.6 \pm 1.9$ & $1200 \pm 120$ & $25 \pm 5$ \\
\hline$Z \rightarrow \tau \tau$ & $1860 \pm 130$ & $144 \pm 26$ & $96100 \pm 2000$ & $274 \pm 33$ \\
Top quark & $1260 \pm 130$ & $390 \pm 34$ & $1620 \pm 210$ & $51 \pm 10$ \\
Misidentified & $1340 \pm 210$ & $41 \pm 21$ & $63900 \pm 1600$ & $149 \pm 33$ \\
Other & $1180 \pm 140$ & $168 \pm 18$ & $23000 \pm 1000$ & $104 \pm 15$ \\
\hline Total background & $5640 \pm 100$ & $743 \pm 29$ & $184500 \pm 1200$ & $580 \pm 30$ \\
Data & 5664 & 723 & 184508 & 583 \\
\hline
\end{tabular}


Candidate background events from data with mass of the $\ell \tau$ system close to $m_{H}=$ $125 \mathrm{GeV}$ are shown in Figures 8.23 and 8.24 for $H \rightarrow e \tau$ and $H \rightarrow \mu \tau$ searches, respectively.
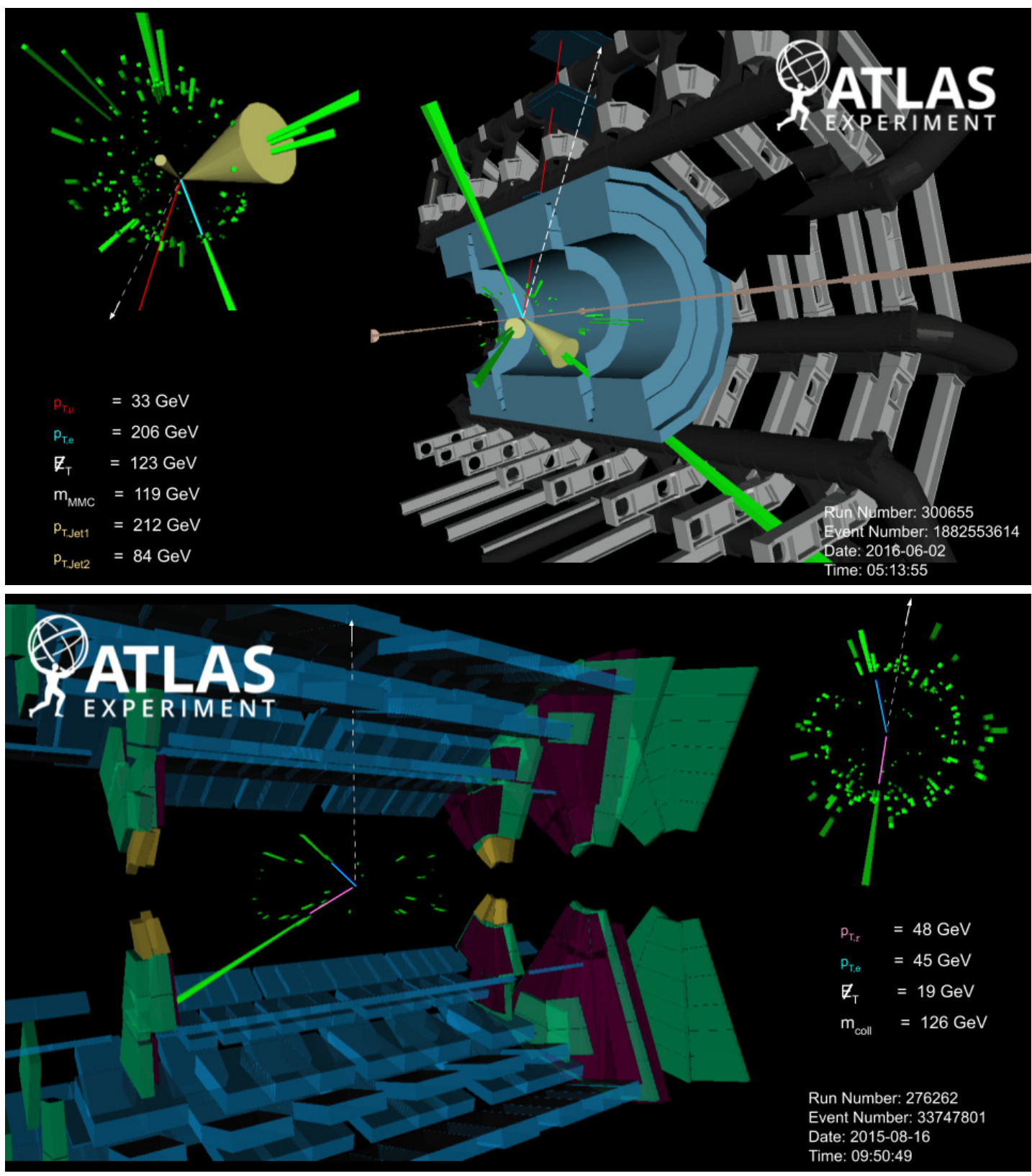

Figure 8.23: Candidate events of the $H \rightarrow e \tau$ search: $e \tau_{\mu}$ (top) and $e \tau_{\text {had }}$ (bottom). An electron track is shown in blue, a red line indicates a muon. A $\tau_{\text {had }}$ candidate is displayed in purple, jets are displayed as dark-yellow cones, the $E_{\mathrm{T}}^{\text {miss }}$ is shown by a white dashed line. 

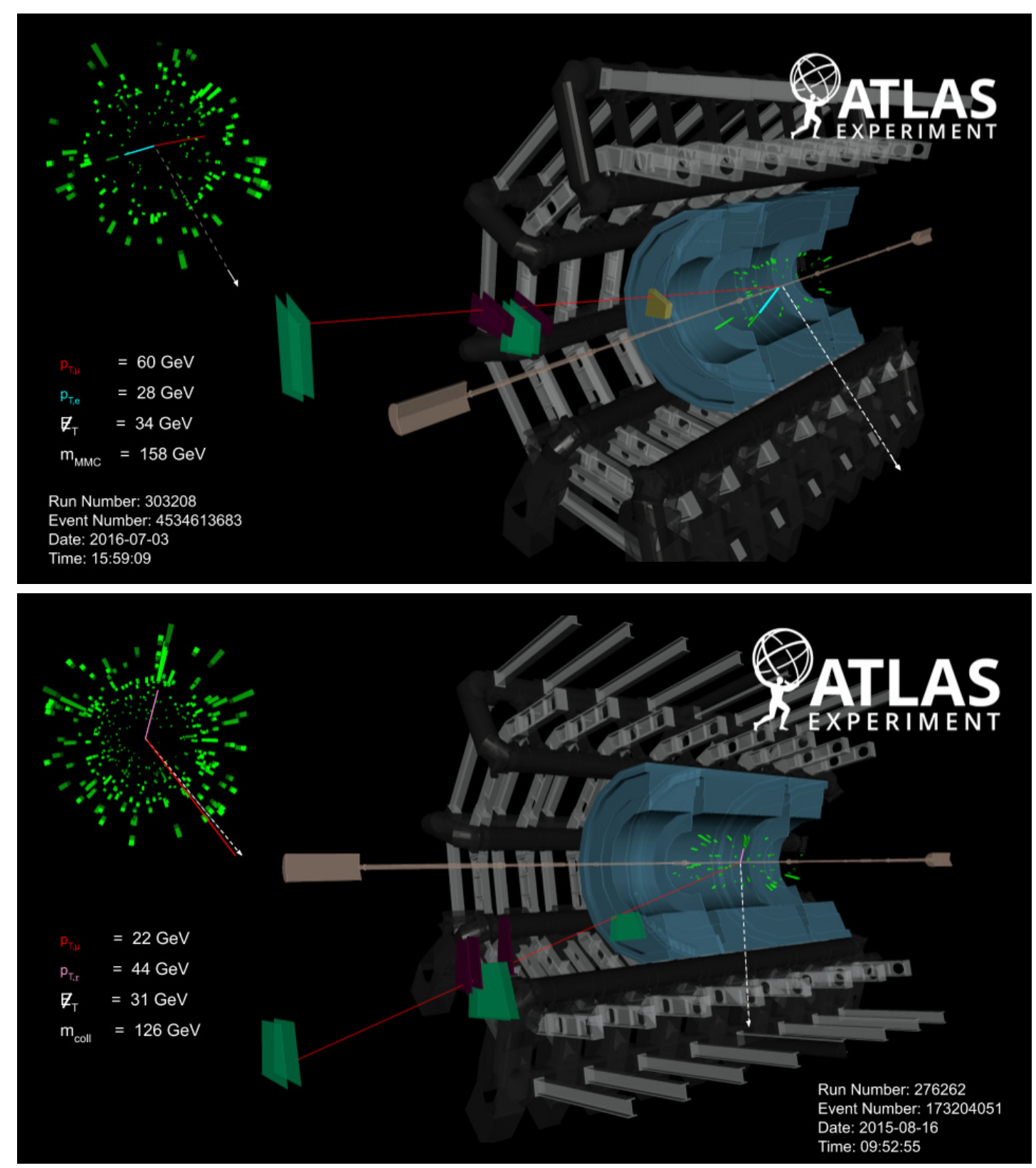

Figure 8.24: Candidate events of the $H \rightarrow \mu \tau$ search: $\mu \tau_{e}$ (top) and $\mu \tau_{\text {had }}$ (bottom). An electron track is shown in blue, a red line indicates a muon. A $\tau_{\text {had }}$ candidate is displayed in purple, the $E_{\mathrm{T}}^{\text {miss }}$ is shown by a white dashed line. 
The best-fit branching ratios and upper limits are computed while assuming $\mathcal{B}(H \rightarrow \mu \tau)=0$ for the $H \rightarrow e \tau$ search and $\mathcal{B}(H \rightarrow e \tau)=0$ for the $H \rightarrow \mu \tau$ search. The best-fit values of the LFV Higgs boson branching ratios are equal to $\left(0.15_{-0.17}^{+0.18}\right) \%$ and $(-0.22 \pm 0.19) \%$ for the $H \rightarrow e \tau$ and $H \rightarrow \mu \tau$ search, respectively. In the absence of a significant excess, upper limits on the LFV branching ratios are set for a Higgs boson with $m_{H}=125 \mathrm{GeV}$. The observed (median expected) $95 \%$ CL upper limits are $0.47 \%\left(0.34_{-0.10}^{+0.13} \%\right)$ and $0.28 \%\left(0.37_{-0.10}^{+0.14} \%\right)$ for the $H \rightarrow e \tau$ and $H \rightarrow \mu \tau$ searches, respectively. These limits are significantly lower than the corresponding Run 1 limits of Refs. [141, 142]. The breakdown of contributions from different signal regions is shown in Figure 8.25, which also compares these results to the results on 95\% CL upper limits and best fit values for the LFV decays of the Higgs boson $\mathrm{BR}(H \rightarrow e \tau)$ and $\mathrm{BR}(H \rightarrow \mu \tau)$ obtained during Run 1 at $8 \mathrm{TeV}$ with $20.3 \mathrm{fb}^{-1}$ of data by the ATLAS experiment [74].
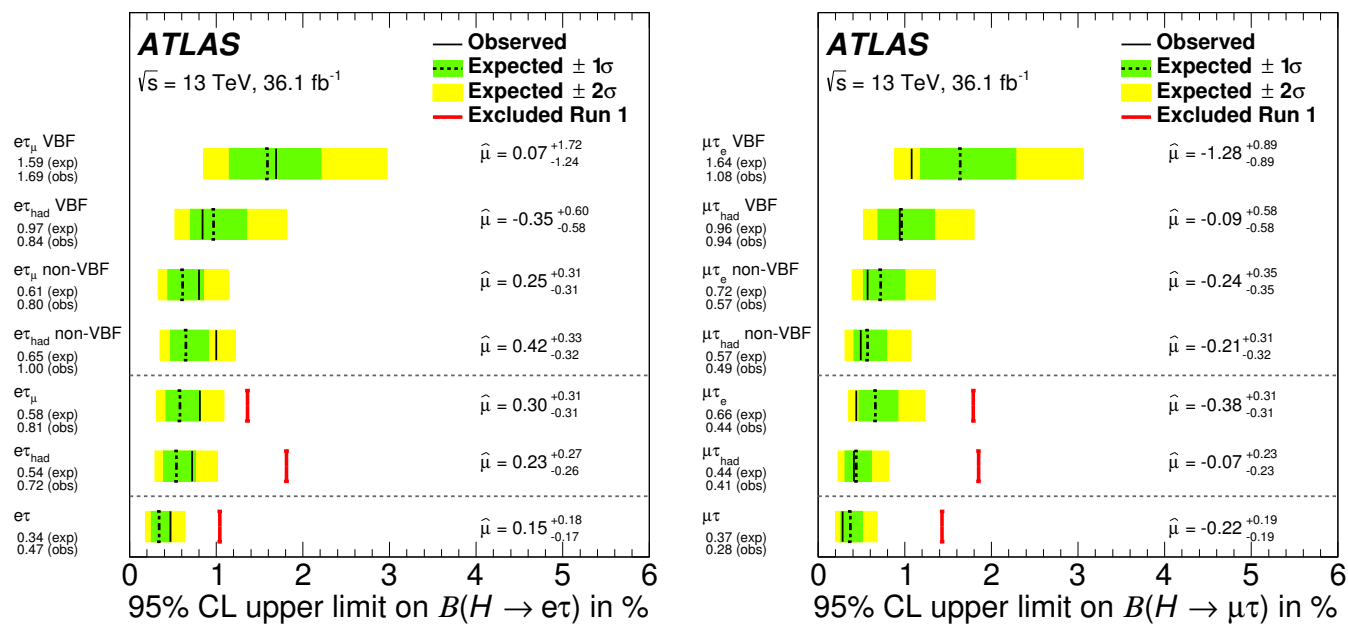

Figure 8.25: Upper limits at 95\% CL on the LFV branching ratios of the Higgs boson, $H \rightarrow e \tau$ (left) and $H \rightarrow \mu \tau$ (right), indicated by solid and dashed lines. Bestfit values of the branching ratios $(\hat{\mu})$ are also given, in $\%$. The limits are computed while assuming that either $\mathcal{B}(H \rightarrow \mu \tau)=0$ (left) or $\mathcal{B}(H \rightarrow e \tau)=0$ (right). First, the results of the fits are shown, when only the data of an individual channel or of an individual category are used; in these cases the signal and control regions from all other channels/categories are removed from the fit. These results are finally compared with the full fit displayed in the last row. Run 1 results are also shown in red. 


\subsection{Conclusions}

The search for $H \rightarrow \mu \tau$ and $H \rightarrow e \tau$ decays with the ATLAS detector at the LHC using $13 \mathrm{TeV}$ data was presented in this thesis. No excess is observed and the $95 \% \mathrm{CL}$ limits based on cut-based analysis for $\mathrm{BR}(H \rightarrow \ell \tau)$ are found to be $0.43 \%$ (exp. $0.49_{-0.14}^{+0.19 \%}$ ) and $0.41 \%$ (exp. $0.45_{-0.13}^{+0.18} \%$ ) for the $H \rightarrow e \tau$ and $H \rightarrow \mu \tau$ searches, respectively, based on $\sqrt{s}=13 \mathrm{TeV}$ of data collected during 2015 and 2016 . Following a review of the internal supporting document [77], these results have been published by the ATLAS experiment [143].

The analysis is found to be three times more sensitive than the previous analysis, and very similar to that obtained by the CMS experiment [76]. The main improvements come from the additional kinematic regions used in the analysis with higher sensitivity to signal, and improved technique employed to model the fake-background with a data-driven methods using a transfer factor from control region to signal region. Further improvements are obtained by inclusion of a new phase-space region corresponding to Vector Boson Fusion production mechanism of the Higgs boson, as well as use of multi-variate signal-to-background discriminants. The result is already systematic error dominated, as shown in Table 8.13. Thus, it will not improve by a huge factor when more data is added to such analysis during further data-taking at the the Large Hadron Collider.

The branching ratio of the LFV Higgs boson decay is related to the non-diagonal Yukawa coupling matrix elements [23] by the formula

$$
\left|Y_{\ell \tau}\right|^{2}+\left|Y_{\tau \ell}\right|^{2}=\frac{8 \pi}{m_{H}} \frac{\mathcal{B}(H \rightarrow \ell \tau)}{1-\mathcal{B}(H \rightarrow \ell \tau)} \Gamma_{H}(\mathrm{SM})
$$

where $\Gamma_{H}(\mathrm{SM})=4.07 \mathrm{MeV}$ [24] stands for the Higgs boson width as predicted by the Standard Model.

Thus, the observed limits on the branching ratio correspond to the following limits 
on the coupling matrix elements: $\sqrt{\left|Y_{\tau e}\right|^{2}+\left|Y_{e \tau}\right|^{2}}<0.0020$, and $\sqrt{\left|Y_{\tau \mu}\right|^{2}+\left|Y_{\mu \tau}\right|^{2}}<$ 0.0015 .

The limits on the branching ratios can also be expressed as the limits on the off-diagonal Yukawa couplings, $Y_{i j}$ [23], where $i, j$ denote the different charged leptons. Early experimental limits on LFV involing $\tau$ leptons are expressed as limits on $\sqrt{\left|Y_{\tau \ell}\right|^{2}+\left|Y_{\ell \tau}\right|^{2}}[23]$, as shown in Section 2.8. Limits are usually compared to a naturalness limit: $\left|Y_{\tau \ell} Y_{\ell \tau}\right| v^{2} \lesssim m_{\tau} m_{\ell}$, so that the mass hierachy is preserved without fine tuning of cancellations of various terms in the Lagrangian [144].

Figures 8.26 show the limits on the couplings $Y_{\tau \ell}$ and $Y_{\ell \tau}$ together with the limits from the ATLAS Run 1 analysis and known limits from $\tau \rightarrow \ell \gamma$. The upper limits obtained in the present analysis from direct searches for lepton flavor violating decays of the Higgs boson at the Large Hadron Collider are about twenty-five times lower than the indirect predictions.
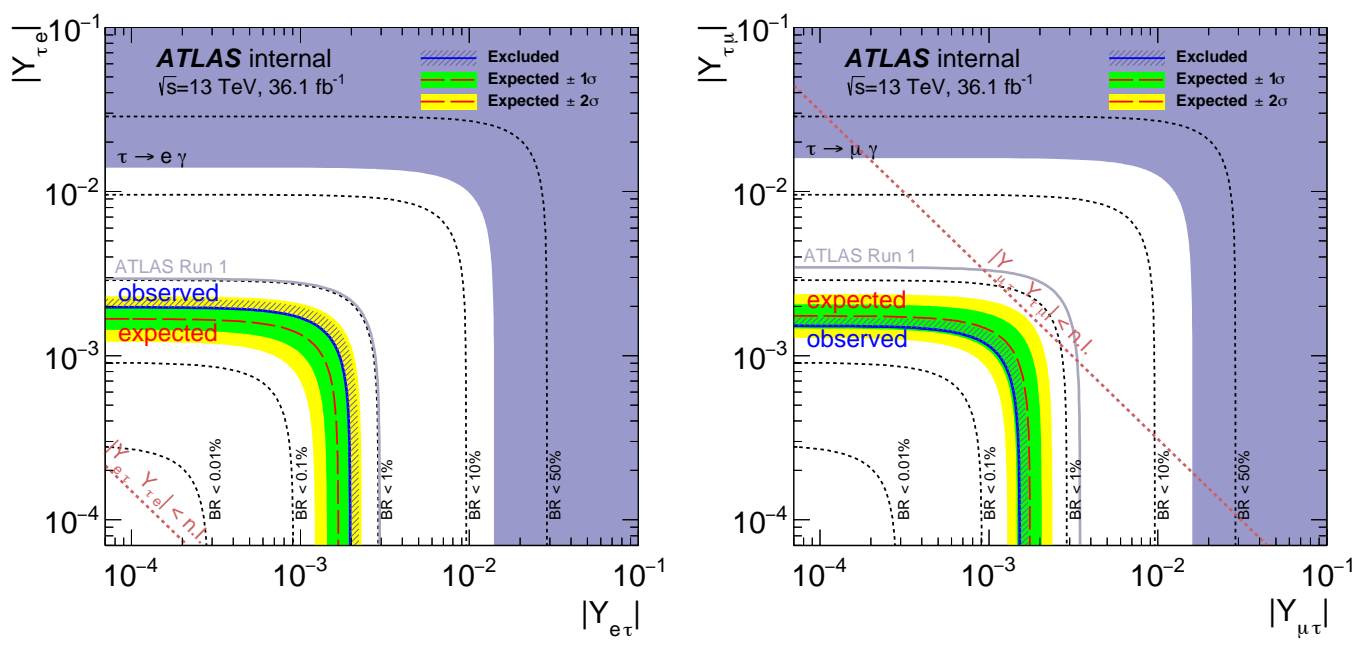

Figure 8.26: Limits on the couplings $Y_{\tau \ell}$ and $Y_{\ell \tau}$ together with the limits from ATLAS Run 1 analysis and known limits from $\tau \rightarrow \ell \gamma$ [23]. Indicated are also limits corresponding to different $B R \mathrm{~s}(0.01 \%, 0.1 \%, 1 \%, 10 \%$ and $50 \%)$ and the naturalness limit $\left|Y_{\tau \ell} Y_{\ell \tau}\right| v^{2} \lesssim m_{\tau} m_{\ell}$. 


\section{REFERENCES}

[1] Emmy Noether. Invariant Variation Problems. Gott. Nachr., 1918:235-257, 1918. doi: $10.1080 / 00411457108231446$.

[2] Paul Adrien Maurice Dirac. Quantised singularities in the electromagnetic field,. Proc. Roy. Soc. Lond. A, A133(821):60-72, 1931. doi: 10.1098/rspa.1931.0130.

[3] Pierre Fayet. Supersymmetry and Weak, Electromagnetic and Strong Interactions. Phys. Lett., B 64:159, 1976. doi: 10.1016/0370-2693(76)90319-1.

[4] S.L. Glashow. Partial Symmetries of Weak Interactions. Nucl. Phys., 22:579588, 1961. doi: 10.1016/0029-5582(61)90469-2.

[5] Steven Weinberg. A Model of Leptons. Phys. Rev. Lett., 19:1264-1266, 1967. doi: 10.1103/PhysRevLett.19.1264.

[6] Abdus Salam. Weak and Electromagnetic Interactions. Conf. Proc. C, 680519: 367-377, 1968. doi: 10.1142/9789812795915\_0034.

[7] Antonio Pich. The Standard model of electroweak interactions. In 2006 European School of High-Energy Physics, pages 1-49, 2008.

[8] Peter W. Higgs. Broken symmetries, massless particles and gauge fields. Phys. Lett., 12:132-133, 1964. doi: 10.1016/0031-9163(64)91136-9.

[9] Peter W. Higgs. Broken Symmetries and the Masses of Gauge Bosons. Phys. Rev. Lett., 13:508-509, 1964. doi: 10.1103/PhysRevLett.13.508. 
[10] F. Englert and R. Brout. Broken Symmetry and the Mass of Gauge Vector Mesons. Phys. Rev. Lett., 13:321-323, 1964. doi: 10.1103/PhysRevLett.13.321.

[11] Abdelhak Djouadi. The Anatomy of electro-weak symmetry breaking. I: The Higgs boson in the standard model. volume 457, pages 1-216, 2008. doi: 10.1016/j.physrep.2007.10.004.

[12] M. Tanabashi et al. Review of Particle Physics. Phys. Rev. D, 98(3):030001, 2018. doi: 10.1103/PhysRevD.98.030001.

[13] David J. Gross and Frank Wilczek. Ultraviolet Behavior of Nonabelian Gauge Theories. Phys. Rev. Lett., 30:1343-1346, 1973. doi: 10.1103/PhysRevLett.30. 1343.

[14] H.David Politzer. Reliable Perturbative Results for Strong Interactions? Phys. Rev. Lett., 30:1346-1349, 1973. doi: 10.1103/PhysRevLett.30.1346.

[15] S. Dittmaier et al. Handbook of LHC Higgs Cross Sections: 1. Inclusive Observables. 1 2011. doi: 10.5170/CERN-2011-002.

[16] D. de Florian et al. Handbook of LHC Higgs Cross Sections: 4. Deciphering the Nature of the Higgs Sector. 2/2017, 10 2016. doi: 10.23731/CYRM-2017-002.

[17] John R. Ellis, Mary K. Gaillard, and Dimitri V. Nanopoulos. A Phenomenological Profile of the Higgs Boson. Nucl.Phys., B106:292, 1976. doi: 10.1016/0550-3213(76)90382-5.

[18] E. Braaten and J.P. Leveille. Higgs Boson Decay and the Running Mass. Phys. Rev. D, 22:715, 1980. doi: 10.1103/PhysRevD.22.715.

[19] LHC Higgs Cross Section Working Group, S. Dittmaier, C. Mariotti, G. Passarino, and R. Tanaka (Eds.). Handbook of LHC Higgs Cross Sections: 2. Differential Distributions. CERN-2012-002, CERN, Geneva, 2012. 
[20] Georges Aad et al. Observation of a new particle in the search for the Standard Model Higgs boson with the ATLAS detector at the LHC. Phys. Lett. B, 716: 1-29, 2012. doi: 10.1016/j.physletb.2012.08.020.

[21] Serguei Chatrchyan et al. Observation of a New Boson at a Mass of $125 \mathrm{GeV}$ with the CMS Experiment at the LHC. Phys. Lett. B, 716:30-61, 2012. doi: 10.1016/j.physletb.2012.08.021.

[22] Gianluca Blankenburg, John Ellis, and Gino Isidori. Flavour-Changing Decays of a 125 GeV Higgs-like Particle. Phys. Lett. B, 712:386-390, 2012. doi: 10. 1016/j.physletb.2012.05.007.

[23] Roni Harnik, Joachim Kopp, and Jure Zupan. Flavor Violating Higgs Decays. JHEP, 1303:026, 2013. doi: 10.1007/JHEP03(2013)026.

[24] LHC Higgs Cross Section Working Group. Handbook of LHC Higgs Cross Sections: 3. Higgs Properties. 2013. doi: 10.5170/CERN-2013-004.

[25] Aubert, Bernard et al., BaBar Collaboration. Searches for Lepton Flavor Violation in the Decays $\tau^{ \pm} \rightarrow e^{ \pm} \gamma$ and $\tau^{ \pm} \rightarrow \mu^{ \pm} \gamma$. Phys. Rev. Lett., 104:021802, 2010. doi: 10.1103/PhysRevLett.104.021802.

[26] LHC Machine. JINST, 3:S08001, 2008. doi: 10.1088/1748-0221/3/08/S08001.

[27] ATLAS Collaboration. Layout of the lhe ring, 1998. URL https:// lhc-machine-outreach.web.cern.ch/images/lhc-schematic.jpg.

[28] J. Barranco Garca, R. De Maria, A. Grudiev, R. Toms Garca, R.B. Appleby, and D.R. Brett. Long term dynamics of the high luminosity Large Hadron Collider with crab cavities. Phys. Rev. Accel. Beams, 19(10):101003, 2016. doi: 10.1103/PhysRevAccelBeams.19.101003. 
[29] ATLAS Collaboration. Luminosity public results run2, 2018. URL https://twiki.cern.ch/twiki/bin/view/AtlasPublic/ LuminosityPublicResultsRun2.

[30] G. Aad et al. The ATLAS Experiment at the CERN Large Hadron Collider. JINST, 3:S08003, 2008. doi: 10.1088/1748-0221/3/08/S08003.

[31] G. Aad et al. Expected Performance of the ATLAS Experiment - Detector, Trigger and Physics. arXiv: 0901.0512 [hep-ex] 2009.

[32] ATLAS Collaboration. Computer generated image for layout of atlas, 1998. URL https://cds. cern. ch/record/39038.

[33] ATLAS Collaboration. Computer generated image of the atlas inner detector, 2008. URL https://cds.cern.ch/record/1095926.

[34] ATLAS inner detector: Technical Design Report, 1. Technical Design Report ATLAS. CERN, Geneva, 1997.

[35] ATLAS Collaboration. Computer generated image of the atlas calorimeter, 2008. URL https://cds. cern.ch/record/1095927?ln=en.

[36] ATLAS liquid-argon calorimeter: Technical Design Report. Technical Design Report ATLAS. CERN, Geneva, 1996.

[37] ATLAS tile calorimeter: Technical Design Report. Technical Design Report ATLAS. CERN, Geneva, 1996.

[38] G. Aad et al. Commissioning of the ATLAS Muon Spectrometer with Cosmic Rays. Eur. Phys. J. C, 70:875-916, 2010. doi: 10.1140/epjc/s10052-010-1415-2.

[39] J.C. Vermeulen et al. The baseline dataflow system of the ATLAS trigger and DAQ. 102003. 
[40] ATLAS Collaboration. Trigger Menu in 2017. ATL-DAQ-PUB-2018-002, 2018. URL https://cds. cern. ch/record/2625986.

[41] CERN, LHC programme. The high-luminosity lhc (hl-lhc) project. URL https: //cds.cern.ch/record/2199189/files/English.pdf.

[42] Expected Tracking Performance of the ATLAS Inner Tracker at the HL-LHC. 2019.

[43] ATLAS Collaboration. Technical Design Report for the ATLAS Inner Tracker Pixel Detector. CERN-LHCC-2017-021, ATLAS-TDR-030, 2017. URL http: //cds. cern. ch/record/2285585/files/ATLAS-TDR-030. pdf?version=2.

[44] Paolo Nason. A new method for combining NLO QCD with shower Monte Carlo algorithms. JHEP, 11:040, 2004. doi: 10.1088/1126-6708/2004/11/040.

[45] S. Frixione, P. Nason and C. Oleari. Matching NLO QCD computations with parton shower simulations: the POWHEG method. JHEP, 11:070, 2007. doi: $10.1088 / 1126-6708 / 2007 / 11 / 070$.

[46] S. Alioli, P. Nason, C. Oleari and E. Re. A general framework for implementing NLO calculations in shower Monte Carlo programs: the POWHEG BOX. JHEP, 06:043, 2010. doi: 10.1007/JHEP06(2010)043.

[47] E. Bagnaschi, G. Degrassi, P. Slavich and A. Vicini. Higgs production via gluon fusion in the POWHEG approach in the SM and in the MSSM. JHEP, 02:088, 2012. doi: 10.1007/JHEP02(2012)088.

[48] K. Hamilton, P. Nason, E. Re and G. Zanderighi. NNLOPS simulation of Higgs boson production. JHEP, 10:222, 2013. doi: 10.1007/JHEP10(2013)222.

[49] K. Hamilton, P. Nason and G. Zanderigh. Finite quark-mass effects in the 
NNLOPS POWHEG+MiNLO Higgs generator. JHEP, 05:140, 2015. doi: 10. 1007/JHEP05(2015)140.

[50] W. Altmannshofer et al. The Belle II Physics Book. PTEP, 2019(12):123C01, 2019. doi: 10.1093/ptep/ptz106. [Erratum: PTEP 2020, 029201 (2020)].

[51] T. Abe. Belle II Technical Design Report. 2010.

[52] J. Kemmer et al. LOW CAPACITY DRIFT DIODE. Nucl. Instrum. Meth., A253:378-381, 1987. doi: 10.1016/0168-9002(87)90519-5.

[53] P. Fischer et al. Progress towards a large area, thin DEPFET detector module. Nucl. Instrum. Meth., A582:843-848, 2007. doi: 10.1016/j.nima.2007.07.108.

[54] M. Akatsu et al. Time of propagation Cherenkov counter for particle identification. Nucl. Instrum. Meth., A440:124-135, 2000. doi: 10.1016/S0168-9002(99) 00819-0.

[55] M. Staric et al. Likelihood analysis of patterns in a time-of-propagation (TOP) counter. Nucl. Instrum. Meth., A595:252-255, 2008. doi: 10.1016/j.nima.2008. 07.018.

[56] M. Akatsu et al. MCP-PMT timing property for single photons. Nucl. Instrum. Meth., A528:763-775, 2004. doi: 10.1016/j.nima.2004.04.207.

[57] H. Kichimi et al. KEKB Beam Collision Stability at the Picosecond Timing and Micron Position Resolution as observed with the Belle Detector. JINST, 5:P03011, 2010. doi: 10.1088/1748-0221/5/03/P03011.

[58] S. Nishida et al. Development of an HAPD with 144 channels for the aerogel RICH of the Belle upgrade. Nucl. Instrum. Meth., A595:150-153, 2008. doi: 10.1016/j.nima.2008.07.032. 
[59] S. Nishida et al. Aerogel RICH for the Belle II forward PID. Nucl. Instrum. Meth., A766:28-31, 2014. doi: 10.1016/j.nima.2014.06.061.

[60] A. Kuzmin. Endcap calorimeter for SuperBelle based on pure CsI crystals. Nucl. Instrum. Meth., A623:252-254, 2010. doi: 10.1016/j.nima.2010.02.212.

[61] A.J. Bevan et al. The Physics of the B Factories. Eur. Phys. J. C, 74:3026, 2014. doi: 10.1140/epjc/s10052-014-3026-9.

[62] V. Balagura et al. Study of scintillator strip with wavelength shifting fiber and silicon photomultiplier. Nucl. Instrum. Meth., A564:590-596, 2006. doi: 10.1016/j.nima.2006.04.030.

[63] T. Aushev et al. A scintillator based endcap $K_{L}$ and muon detector for the Belle II experiment. Nucl. Instrum. Meth. A, 789:134-142, 2015. doi: 10.1016/ j.nima.2015.03.060.

[64] A Abashian et al. Muon identification in the Belle experiment at KEKB. Nucl. Instrum. Meth. A, 491:69-82, 2002. doi: 10.1016/S0168-9002(02)01164-6.

[65] J.G. Wang. RPC performance at KLM / BELLE. Nucl. Instrum. Meth. A, 508: 133-136, 2003. doi: 10.1016/S0168-9002(03)01335-4.

[66] Belle II Collaboration. Klm detector. https://confluence.desy.de/ display/BI/KLM+DAQ+-+short+and+easy+introduction.

[67] P.A. Zyla et al. Review of Particle Physics. Prog. Theor. Exp. Phys, 2020 (083C01), 2020.

[68] S. Jadach, B.F.L. Ward, and Z. Was. The Precision Monte Carlo event generator K K for two fermion final states in e+ e- collisions. Comput. Phys. Commun., 130:260-325, 2000. doi: 10.1016/S0010-4655(00)00048-5. 
[69] S. Jadach, Z. Was, R. Decker, and Johann H. Kuhn. The tau decay library TAUOLA: Version 2.4. Comput. Phys. Commun., 76:361-380, 1993. doi: 10. 1016/0010-4655(93)90061-G.

[70] Torbjorn Sjostrand, Stephen Mrenna, and Peter Z. Skands. PYTHIA 6.4 Physics and Manual. JHEP, 05:026, 2006. doi: 10.1088/1126-6708/2006/05/ 026.

[71] M. Chrzaszcz, T. Przedzinski, Z. Was, and J. Zaremba. TAUOLA of $\tau$ lepton decaysframework for hadronic currents, matrix elements and anomalous decays. Comput. Phys. Commun., 232:220-236, 2018. doi: 10.1016/j.cpc.2018.05.017.

[72] S. Banerjee and A Pathak. Update of TAUOLA Monte Carlo for Belle II software. BELLE2-NOTE-PH-2020-055, July 2020.

[73] S. Antropov, Sw. Banerjee, Z. Was, and J. Zaremba. TAUOLA Update for Decay Channels with $e^{+} e^{-}$Pairs in the Final State. 1912.113762019.

[74] ATLAS Collaboration. Search for lepton-flavour-violating decays of the Higgs and $Z$ bosons with the ATLAS detector.

[75] CMS Collaboration. Search for Lepton-Flavour-Violating Decays of the Higgs Boson. Phys. Lett. B, 749:337-362, 2015. doi: 10.1016/j.physletb.2015.07.053.

[76] CMS Collaboration. Search for lepton flavour violating decays of the Higgs boson to $\mu \tau$ and $e \tau$ in proton-proton collisions at $\sqrt{s}=13 \mathrm{TeV}$. JHEP, 06:001, 2018. doi: 10.1007/JHEP06(2018)001.

[77] ATLAS Internal Note. Search for lepton-flavour-violating decays of the higgs bosons in 13 tev collisions with the atlas detector. ATL-COM-PHYS-2017-452.

[78] ATLAS Collaboration. The ATLAS simulation infrastructure. Eur. Phys. J., C 70:823-874, 2010. doi: 10.1140/epjc/s10052-010-1429-9. 
[79] GEANT4 Collaboration, S. Agostinelli et al. GEANT4 - a simulation toolkit. Nucl. Instrum. Meth., A 506:250-303, 2003. doi: 10.1016/S0168-9002(03) 01368-8.

[80] ATLAS Collaboration. Combined Measurement of the Higgs Boson Mass in pp Collisions at $\sqrt{s}=7$ and $8 \mathrm{TeV}$ with the ATLAS and CMS Experiments. Phys. Rev. Lett., 114:191803, 2015. doi: 10.1103/PhysRevLett.114.191803.

[81] D. de Florian et al., LHC Higgs Cross Section Working Group. Handbook of LHC Higgs cross sections: 4. Deciphering the nature of the Higgs sector. 2017. doi: 10.23731/CYRM-2017-002.

[82] Torbjrn Sjstrand, Stefan Ask, Jesper R. Christiansen, Richard Corke, Nishita Desai, Philip Ilten, Stephen Mrenna, Stefan Prestel, Christine O. Rasmussen, and Peter Z. Skands. An introduction to PYTHIA 8.2. Comput. Phys. Commun., 191:159-177, 2015. doi: 10.1016/j.cpc.2015.01.024.

[83] ATLAS Collaboration. Cross-section measurements of the Higgs boson decaying to a pair of tau leptons in proton-proton collisions at $\sqrt{s}=13 \mathrm{TeV}$ with the ATLAS Detector. Phys. Rev. D, 99:072001, 2018. doi: 10.1103/PhysRevD.99. 072001.

[84] Paolo Nason. A New method for combining NLO QCD with shower Monte Carlo algorithms. JHEP, 11:040, 2004. doi: 10.1088/1126-6708/2004/11/040.

[85] Jon Butterworth et al. PDF4LHC recommendations for LHC Run II. J. Phys. G, 43:023001, 2016. doi: 10.1088/0954-3899/43/2/023001.

[86] C. Anastasiou, C. Duhr, F. Dulat, F. Herzog and B. Mistlberger. Higgs Boson Gluon-Fusion Production in QCD at Three Loops . Phys. Rev. Lett., 114: 212001, 2015. doi: 10.1103/PhysRevLett.114.212001. 
[87] C. Anastasiou et al. High precision determination of the gluon fusion Higgs boson cross-section at the LHC. JHEP, 05:058, 2016. doi: 10.1007/JHEP05(2016) 058.

[88] S. Actis, G. Passarino, C. Sturm and S. Uccirati. NLO electroweak corrections to Higgs boson production at hadron colliders. Phys. Lett. B, 670:12, 2008. doi: 10.1016/j.physletb.2008.10.018.

[89] C. Anastasiou, R. Boughezal and F. Petriello. Mixed QCD-electroweak corrections to Higgs boson production in gluon fusion. JHEP, 04:003, 2009. doi: 10.1088/1126-6708/2009/04/003.

[90] M. Ciccolini, A. Denner and S. Dittmaier. Strong and Electroweak Corrections to the Production of a Higgs Boson +2 Jets via Weak Interactions at the Large Hadron Collider. Phys. Rev. Lett., 99:161803, 2007. doi: 10.1103/PhysRevLett. 99.161803.

[91] M. Ciccolini, A. Denner and S. Dittmaier. Electroweak and QCD corrections to Higgs production via vector-boson fusion at the LHC. Phys. Rev. D, 77:013002, 2008. doi: 10.1103/PhysRevD.77.013002.

[92] P. Bolzoni, F. Maltoni, S.-O. Moch and M. Zaro. Higgs Boson Production via Vector-Boson Fusion at Next-to-Next-to-Leading Order in QCD. Phys. Rev. Lett., 105:011801, 2010. doi: 10.1103/PhysRevLett.105.011801.

[93] O. Brein, A. Djouadi and R. Harlander. NNLO QCD corrections to the Higgsstrahlung processes at hadron colliders. Phys. Lett. B, 579:149, 2004. doi: 10.1016/j.physletb.2003.10.112.

[94] L. Altenkamp, S. Dittmaier, R. V. Harlander, H. Rzehak and T. J. E. Zirke. Gluon-induced Higgs-strahlung at next-to-leading order QCD. JHEP, 02:078, 2013. doi: 10.1007/JHEP02(2013)078. 
[95] A. Denner, S. Dittmaier, S. Kallweit and A. Mück. Electroweak corrections to Higgs-strahlung off $\mathrm{W} / \mathrm{Z}$ bosons at the Tevatron and the LHC with Hawk. JHEP, 03:075, 2012. doi: 10.1007/JHEP03(2012)075.

[96] T. Gleisberg, Stefan. Hoeche, F. Krauss, M. Schonherr, S. Schumann, F. Siegert, and J. Winter. Event generation with SHERPA 1.1. JHEP, 02:007, 2009. doi: 10.1088/1126-6708/2009/02/007.

[97] Richard D. Ball et al. Parton distributions for the LHC Run II. JHEP, 04:040, 2015. doi: 10.1007/JHEP04(2015)040.

[98] S. Schumann and F. Krauss. A parton shower algorithm based on CataniSeymour dipole factorisation. JHEP, 03:038, 2008. doi: 10.1088/1126-6708/ 2008/03/038.

[99] Kirill Melnikov and Frank Petriello. Electroweak gauge boson production at hadron colliders through $\mathrm{O}($ alpha(s)**2). Phys. Rev. D, 74:114017, 2006. doi: 10.1103/PhysRevD.74.114017.

[100] Charalampos Anastasiou, Lance J. Dixon, Kirill Melnikov, and Frank Petriello. High precision QCD at hadron colliders: Electroweak gauge boson rapidity distributions at NNLO. Phys. Rev., D 69:094008, 2004. doi: 10.1103/PhysRevD. 69.094008 .

[101] S. Alioli, S.-O. Moch and P. Uwer. Hadronic top-quark pair-production with one jet and parton showering. JHEP, 01:137, 2012. doi: 10.1007/JHEP01(2012)137.

[102] Hung-Liang Lai et al. New parton distributions for collider physics. 2010.

[103] M. Czakon and A. Mitov. Top++: A program for the calculation of the top-pair cross-section at hadron colliders. Comput. Phys. Commun., 185:2930, 2014. doi: 10.1016/j.cpc.2014.06.021. 
[104] S. Alioli, P. Nason, C. Oleari and E. Re. NLO single-top production matched with shower in POWHEG: s- and t-channel contributions. JHEP, 09:111, 2009. doi: 10.1088/1126-6708/2009/09/111.

[105] E. Re. Single-top Wt-channel production matched with parton showers using the POWHEG method. Eur. Phys. J. C, 71:1547, 2011. doi: 10.1140/epjc/ s10052-011-1547-z.

[106] M. Aliev et al. HATHOR - HAdronic Top and Heavy quarks crOss section calculatoR. Comput. Phys. Commun., 182:1034, 2011. doi: 10.1016/j.cpc.2010. 12.040 .

[107] P. Kant et al. HATHOR for single top-quark production: Updated predictions and uncertainty estimates for single top-quark production in hadronic collisions. Comput. Phys. Commun., 191:74, 2015. doi: 10.1016/j.cpc.2015.02.001.

[108] N. Kidonakis. Two-loop soft anomalous dimensions for single top quark associated production with a $W^{-}$or $H^{-}$. Phys. Rev. D, 82:054018, 2010. doi: 10.1103/PhysRevD.82.054018.

[109] Morad Aaboud et al. Electron reconstruction and identification in the ATLAS experiment using the 2015 and 2016 LHC proton-proton collision data at $\sqrt{s}=$ 13 TeV. Eur. Phys. J. C, 79(8):639, 2019. doi: 10.1140/epjc/s10052-019-7140-6.

[110] Georges Aad et al. Muon reconstruction performance of the ATLAS detector in proton-proton collision data at $\sqrt{s}=13 \mathrm{TeV}$. Eur. Phys. J. C, 76(5):292, 2016. doi: 10.1140/epjc/s10052-016-4120-y.

[111] Matteo Cacciari, Gavin P. Salam, and Gregory Soyez. The Anti- $k_{t}$ jet clustering algorithm. JHEP, 0804:063, 2008. doi: 10.1088/1126-6708/2008/04/063. 
[112] Matteo Cacciari, Gavin P. Salam, and Gregory Soyez. FastJet User Manual. Eur. Phys. J., C 72:1896, 2012. doi: 10.1140/epjc/s10052-012-1896-2.

[113] ATLAS Collaboration. Topological cell clustering in the ATLAS calorimeters and its performance in LHC Run 1. Eur. Phys. J. C, 77:490, 2017. doi: 10. 1140/epjc/s10052-017-5004-5.

[114] ATLAS Collaboration. Tagging and suppression of pileup jets with the ATLAS detector. ATLAS-CONF-2014-018, 2014. URL https://cds.cern.ch/ record/1700870.

[115] ATLAS Collaboration. Identification and rejection of pile-up jets at high pseudorapidity with the ATLAS detector. Eur. Phys. J. C, 77:580, 2017. doi: 10.1140/epjc/s10052-017-5081-5.

[116] ATLAS Collaboration. Performance of $b$-jet identification in the ATLAS experiment. JINST, 11:P04008, 2016. doi: 10.1088/1748-0221/11/04/P04008.

[117] ATLAS Collaboration. Optimisation of the ATLAS $b$-tagging performance for the 2016 LHC Run. ATL-PHYS-PUB-2016-012, 2016. URL https://cds. cern.ch/record/2160731.

[118] ATLAS Collaboration. Reconstruction, Energy Calibration, and Identification of Hadronically Decaying Tau Leptons in the ATLAS Experiment for Run-2 of the LHC. ATL-PHYS-PUB-2015-045, 2015. URL https://cds.cern.ch/ record/2064383.

[119] ATLAS Collaboration. Measurement of the tau lepton reconstruction and identification performance in the ATLAS experiment using $p p$ collisions at $\sqrt{s}=13 \mathrm{TeV}$. ATLAS-CONF-2017-029, 2017. URL https://cds.cern.ch/ record/2261772. 
[120] L. Breiman, J. Friedman, R. Olshen, and C. Stone. Classification and Regression Trees. Chapman \& Hall, New York, 1984.

[121] Yoav Freund and Robert E. Schapire. A decision-theoretic generalization of on-line learning and an application to boosting. J. Comput. Syst. Sci., 55:119, 1997. ISSN 0022-0000. doi: 10.1006/jcss.1997.1504.

[122] J. Friedman. Stochastic gradient boosting. Comput. Stat. Data Anal., 38:367, 2002. doi: 10.1016/S0167-9473(01)00065-2.

[123] ATLAS Collaboration. Performance of Missing Transverse Momentum Reconstruction in ATLAS studied in Proton-Proton Collisions recorded in 2012 at $8 \mathrm{TeV}$. ATLAS-CONF-2013-082, 2013. URL https://cds.cern.ch/record/ 1570993.

[124] A. Elagin, P. Murat, A. Pranko, and A. Safonov. A New Mass Reconstruction Technique for Resonances Decaying to di-tau. Nucl. Instrum. Meth. A, 654: 481-489, 2011. doi: 10.1016/j.nima.2011.07.009.

[125] Andreas Hoecker et al. TMVA - Toolkit for Multivariate Data Analysis, 2007.

[126] T. Chen and C. Guestrin. Xgboost: A scalable tree boosting system. In Proceedings of the 22nd ACM SIGKDD International Conference on Knowledge Discovery and Data Mining, pages 785-794, 2016. doi: 10.1145/2939672.2939785. URL https://dl .acm.org/citation. cfm?doid=2939672.2939785.

[127] Morad Aaboud et al. Performance of the ATLAS Trigger System in 2015. Eur. Phys. J. C, 77(5):317, 2017. doi: 10.1140/epjc/s10052-017-4852-3.

[128] ATLAS Collaboration. Trigger Menu in 2016. ATL-DAQ-PUB-2017-001, 2017. URL https://cds. cern. ch/record/2242069. 
[129] ATLAS Collaboration. Measurement of the $H \rightarrow \tau^{+} \tau-$ cross-section in $13 \mathrm{TeV}$ Collisions with the ATLAS Detector. (ATL-COM-PHYS-2017-446), 2016. URL https://cds.cern.ch/record/2261605.

[130] Luminosity determination in pp collisions at $\sqrt{s}=7$ tev using the atlas detector in 2011.

[131] Michal Czakon and Alexander Mitov. Top++: A Program for the Calculation of the Top-Pair Cross-Section at Hadron Colliders. Comput. Phys. Commun., 185:2930, 2014. doi: 10.1016/j.cpc.2014.06.021.

[132] M. Aliev, H. Lacker, U. Langenfeld, S. Moch, P. Uwer, et al. HATHOR: HAdronic Top and Heavy quarks crOss section calculatoR. Comput.Phys.Commun., 182:1034-1046, 2011. doi: 10.1016/j.cpc.2010.12.040.

[133] P. Kant, O.M. Kind, T. Kintscher, T. Lohse, T. Martini, S. Molbitz, P. Rieck, and P. Uwer. HatHor for single top-quark production: Updated predictions and uncertainty estimates for single top-quark production in hadronic collisions. Comput. Phys. Commun., 191:74-89, 2015. doi: 10.1016/j.cpc.2015.02.001.

[134] ATLAS Collaboration. Simulation of top-quark production for the ATLAS experiment at $\sqrt{s}=13 \mathrm{TeV}$. ATL-PHYS-PUB-2016-004, 2016. URL http: //cdsweb.cern.ch/record/2120417.

[135] M. Bahr et al. Herwig++ Physics and Manual. Eur. Phys. J. C, 58:639-707, 2008. doi: 10.1140/epjc/s10052-008-0798-9.

[136] ATLAS Collaboration. Measurement of the $Z / \gamma^{*}$ boson transverse momentum distribution in pp collisions at $\sqrt{s}=7 \mathrm{TeV}$ with the ATLAS detector. JHEP, 09:55, 2014. doi: 10.1007/JHEP09(2014)145. 
[137] Gregory Schott. RooStats for Searches. In PHYSTAT 2011, pages 199-208, Geneva, 2011. CERN. doi: 10.5170/CERN-2011-006.199.

[138] Christian Gumpert, Lorenzo Moneta, Kyle Cranmer, Sven Kreiss, and Wouter Verkerke. Software for statistical data analysis used in Higgs searches. J. Phys. Conf. Ser., 490:012229, 2014. doi: 10.1088/1742-6596/490/1/012229.

[139] J. Glatzer and E. Coniavitis. WorkspaceBuilder - a user-friendly framework to construct RooWorkspace inputs for HistFactory. 2017. doi: https://gitlab.cern. ch/ATauLeptonAnalysiS/WorkspaceBuilder.

[140] Alexander L. Read. Presentation of search results: The CL(s) technique. J. Phys. G, 28:2693-2704, 2002. doi: 10.1088/0954-3899/28/10/313.

[141] ATLAS Collaboration. Search for lepton-flavour-violating $H \rightarrow \mu \tau$ decays of the Higgs boson with the ATLAS detector. JHEP, 11:211, 2015. doi: 10.1007/ JHEP11(2015)211.

[142] ATLAS Collaboration. Search for lepton-flavour-violating decays of the Higgs and $Z$ bosons with the ATLAS detector. Eur. Phys. J. C, 77:70, 2017. doi: 10.1140/epjc/s10052-017-4624-0.

[143] Georges Aad et al. Searches for lepton-flavour-violating decays of the Higgs boson in $\sqrt{s}=13 \mathrm{TeV}$ pp collisions with the ATLAS detector. Phys. Lett. B, 800:135069, 2020. doi: 10.1016/j.physletb.2019.135069.

[144] T. P. Cheng and Marc Sher. Mass-matrix ansatz and flavor nonconservation in models with multiple higgs doublets. Phys. Rev. D, 35:3484-3491, Jun 1987. doi: 10.1103/PhysRevD.35.3484. URL https://link.aps.org/doi/10.1103/ PhysRevD.35.3484. 


\section{CURRICULUM VITA}

NAME:

ADDRESS: $\quad 2310$ Crittenden DR, Apt. 3305D

Louisville, KY 40217, U.S.A.

DOB

WEB:

EDUCATION: B.Sc., Physics

University of Calcutta, India

$2010-13$

M.Sc., Physics

University of Calcutta, India

2013-15

Ph.D., Physics

University of Louisville, USA

2017-20

AWARDS: $\quad$ Arts \& Sciences Undergraduate Mentored Research Award University of Louisville, 2019

Arts \& Sciences Research \& Creative Activities Grant Award University of Louisville, 2018, 2019

West Bengal Merit-cum-means scholarship for Higher Secondary Examination, 2010, 2008 
PUBLICATIONS: Co-authors of ATLAS and Belle II collaborations since November 2017 and October 2019, respectively.

Full list of 238 publications available at here.

Few selected are listed below

- Searches for lepton-flavour-violating decays of the Higgs boson in $\sqrt{\mathrm{s}}$ $=13 \mathrm{TeV}$ pp collisions with the ATLAS detector. Published in Physics Letters B800 (2020), 135069, E-print number: arXiv: 1907.06131, Report number: https://cds.cern.ch/record/2682637

- Technical Design Report for the ATLAS Inner Tracker Pixel Detector.

Report number: ATLAS-TDR-030, http://cdsweb.cern.ch/record/2285585

- Technical Design Report for the ATLAS Inner Tracker Strip Detector.

Report number: ATLAS-TDR-025, http://cdsweb.cern.ch/record/2257755

SKILLS:

- Fortran, C, C++, Python, ROOT (an object oriented Data Analysis Framework).

- Expertise in Shell, Sed, Awk, Perl, Latex, CSS, Javascript, Panda, Scikit-learn, TensorFlow, PyTorch, Grid Computing.

- Data mining, visualization and analysis, Monte Carlo methods, Machine learning, Statistical inference.

- Matrix manipulations, Polynomial expansions, Finite range integrals, Multivariate discriminators.

- In Fall 2018 semester, audited a course on Computer Design and Lab to learn FPGA programming language. 


\section{PROJECTS:}

- Validating the Belle2 PhysicsList in Geant4 v10.1.2.

Undergraduate Arts and Research Showcase. (July 2019 - April 2020 )

https://ir.library.louisville.edu/uars/23/

- Charged particle identification using calorimetry and tracking at the

Belle II experiment.

Undergraduate Arts and Research Showcase.

https://ir.library.louisville.edu/uars/25/

- Data quality monitoring of the performance of KLM detector at the Belle II experiment.

Oct 2018 - July 2020

- Searches of lepton-flavor-violating decays of the Higgs boson with the ATLAS experiment.

Jan 2017 - Jul 2019

- Benchmarking CPU requirments for charged track reconstruction in the ATLAS experiment.

May 2017 - Dec 2018

- Simulation and material modelling for the Inner Tracker Upgrade of the ATLAS experiment.

Feb 2016 -Apr 2017

\section{PRESENTATIONS:}

- Belle II summer workshop at BNL: Expert level diagnostic DQM web-interface for KLM (Jul-Aug 2019).

- DPF Meeting at Fermilab: Searches for lepton-flavor-violating Higgs decays at ATLAS (Jul - Aug, 2017). 
- Lawrence Berkeley National Laboratory: Weekly progress meeting on research on ATLAS (May - Jun, 2016).

- University of Louisville: Weekly progress meeting in High Energy Physics (Feb 2016 onwards).

- Virtual visit to Compact Muon Solenoid detector organized by Saha Institute of Nuclear Physics (Jan 2015).

- C.K. Majumdar memorial summer workshop organized by Indian Association of Physics Teachers (July 2013).

- UGC sponsored national seminars on quantum mechanics \& particle physics (Nov 2011). 